\title{
ANIL INCEL
}

AMINO ACID SEQUENCE AND SIDE CHAIN SPECIFIC SYNTHETIC RECEPTORS TARGETING PROTEIN PHOSPHORYLATION
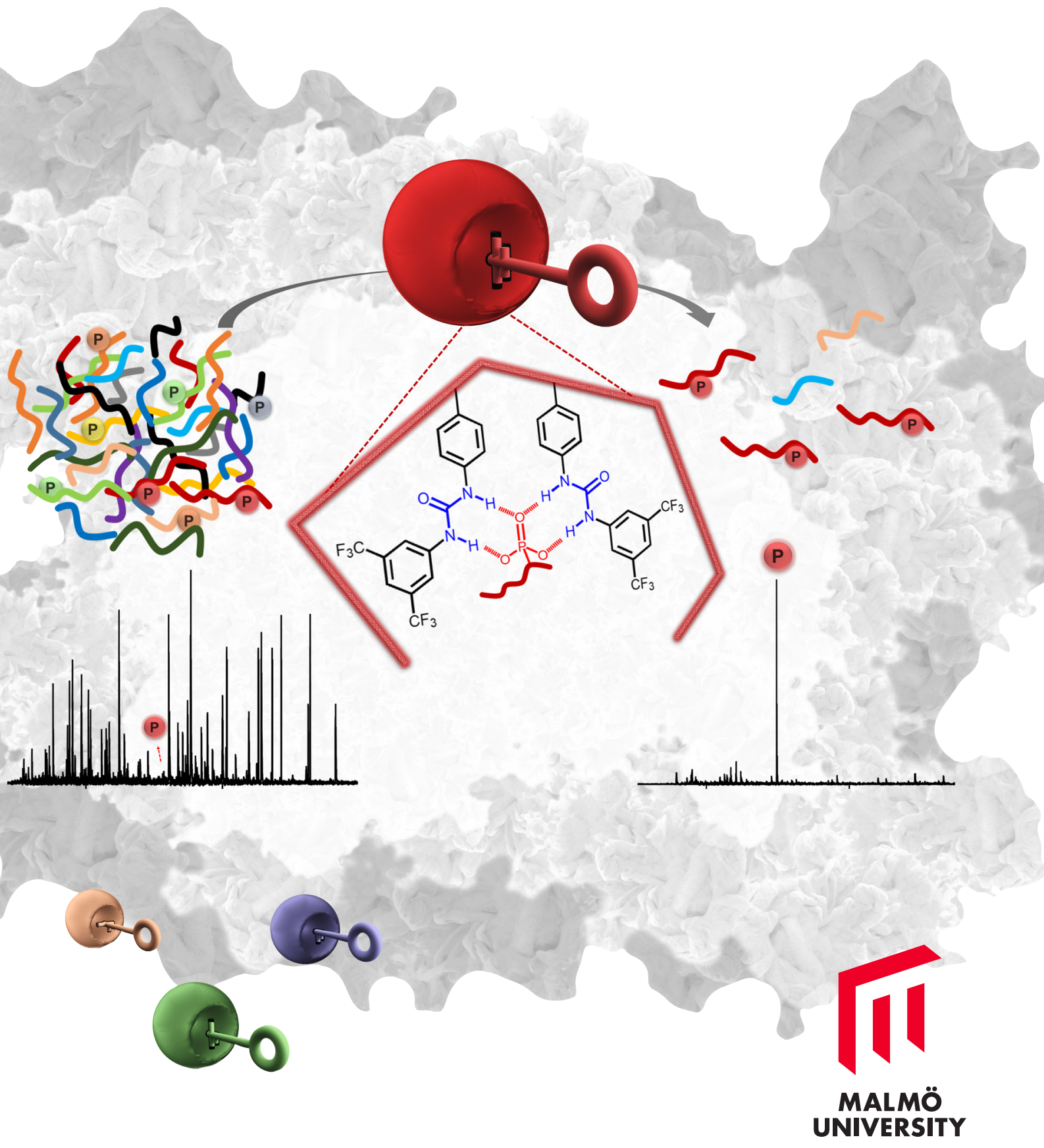

AMINO ACID SEQUENCE AND SIDE CHAIN SPECIFIC SYNTHETIC RECEPTORS

TARGETING PROTEIN PHOSPHORYLATION 


\section{Malmö University, \\ Faculty of Health and Society, Doctoral Dissertation 2021:5}

(C) Copyright An1l Incel 2021

Front Cover: The lock-and-key model: Enrichment of target phosphopeptide using MIPs Back Cover: Fishing phosphorylated targets from complex mixture

ISBN 978-91-7877-207-0 (print)

ISBN 978-91-7877-208-7 (pdf)

ISSN 1653-5383

DOI 10.24834/isbn.9789178772087

Printing: Holmbergs, Malmö 2021 
ANIL INCEL

AMINO ACID SEQUENCE AND SIDE CHAIN SPECIFIC SYNTHETIC RECEPTORS TARGETING PROTEIN PHOSPHORYLATION

Malmö University, 2021

Faculty of Health and Society Department of Biomedical Science 
Dedicated to six years old me...

Remember the ambitious and curiosity that little boy had about nature and science.

If knowledge is power, knowing what we don't know is wisdom...

Adam Grant, Think Again 


\section{CONTENT}

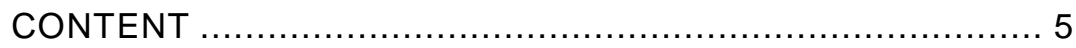

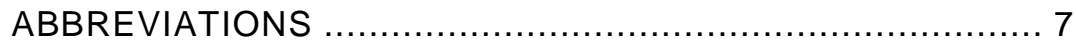

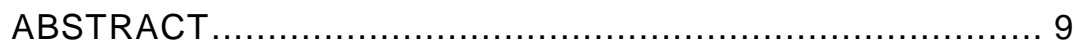

POPULÄRVETENSKAPLIG SAMMANFATTNING ...............10

LIST OF PUBLICATIONS AND CONTRIBUTIONS ..............11

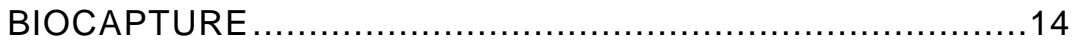

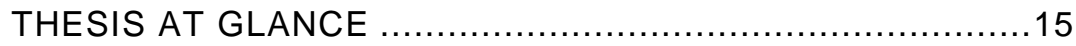

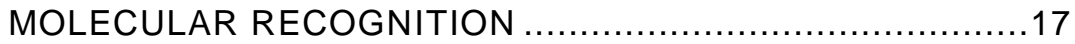

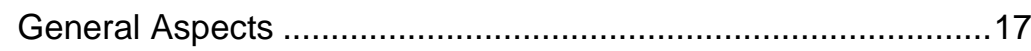

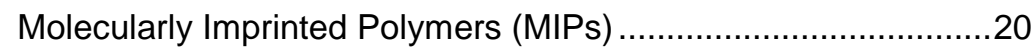

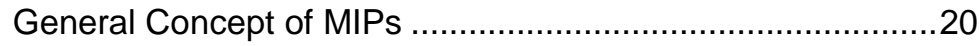

Fundamental Aspects of MIPs..........................................22

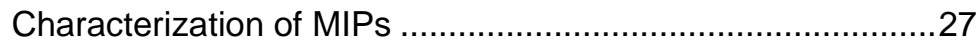

Thermodynamic Principles underlying MIPs formulation .......28

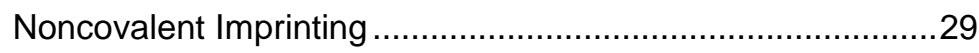

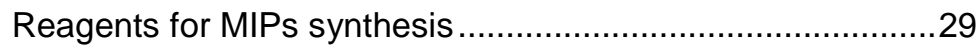

PROTEIN PHOSPHORYLATION ................................... 33

Proteomics and Post-Translational Modifications (PTMs) ............33

Phosphorylation as a PTM.................................................... 34

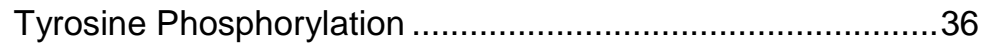

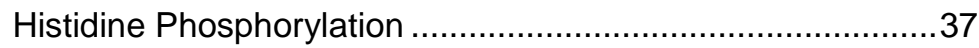

The Role and Significance of Phosphorylation in Cancer .......39

Enrichment and Characterization of Protein Phosphorylation .....42

Enrichment Strategies ..........................................................4 44

Characterization - MS-based phosphoproteomics ................48

MIPS FOR PHOSPHOPEPTIDE ENRICHMENT ..................55

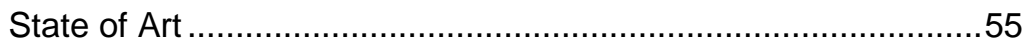




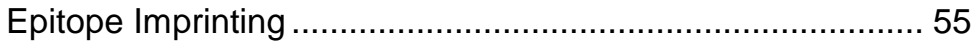

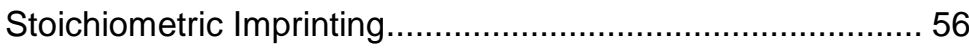

Anion Binding with Urea and Thiourea Receptors................. 56

Urea-based Functional Monomers........................................... 58

Oxoanion-oxourea Complexation ............................................6 60

Side Chain- and Sequence- Specific Imprinted Polymers .......... 62

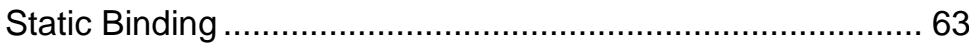

Enrichment of Target Phosphopeptides .............................. 64

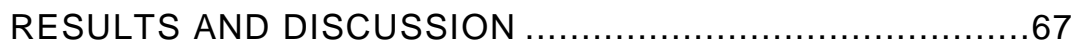

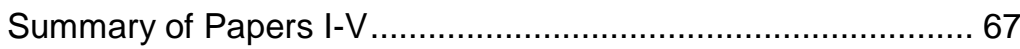

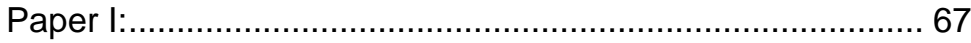

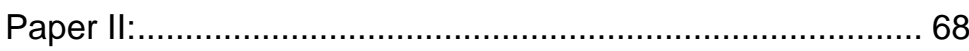

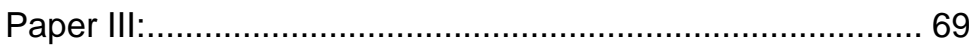

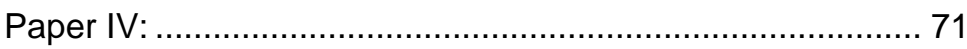

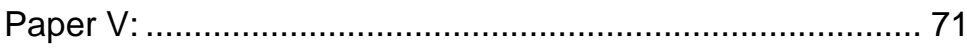

CONCLUDING REMARKS AND FUTURE OUTLOOK ...........73

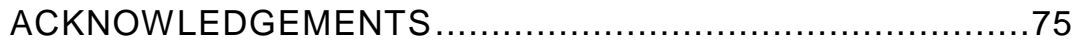

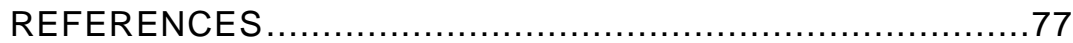

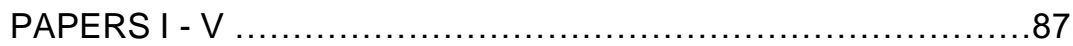




\section{ABBREVIATIONS}

\begin{tabular}{ll} 
ATP & Adenosine triphosphate \\
BSA & Bovine serum albumin \\
CID & Collision induced dissociation \\
CLL & Chronic lymphocytic leukemia \\
DNA & Deoxyribonucleic acid \\
ECD & Electron capture dissociation \\
ESI & Electrospray ionization \\
ETD & Electron transfer dissociation \\
HCD & High-energy collisional dissociation \\
HG & Host guest \\
HILIC & Hydrophilic interaction chromatography \\
HPLC & High-performance liquid chromatography \\
IMAC & Immobilized metal ion affinity chromatography \\
IP & Immunoprecipitation \\
LC & Liquid chromatography \\
MALDI & Matrix-assisted laser desorption/ionization \\
MeCN & Acetonitrile \\
MIP & Molecularly imprinted polymer \\
MOAC & Metal oxide affinity chromatography \\
MS & Mass spectrometry \\
NIP & Non-imprinted polymer \\
NMR & Nuclear magnetic resonance \\
PHis & Phosphohistidine \\
\hline
\end{tabular}




$\begin{array}{ll}\text { pSer } & \text { Phosphoserine } \\ \text { pThr } & \text { Phosphothreonine } \\ \text { PTM } & \text { Post-translational modification } \\ \text { pTyr } & \text { Phosphotyrosine } \\ \text { pTza } & \text { Phosphoryltriazolylalanine } \\ \text { SAX } & \text { Strong anion exchange } \\ \text { SCX } & \text { Strong cation exchange } \\ \text { SH2 } & \text { Src Homology 2 } \\ \text { SIMAC } & \text { Sequential elution from IMAC } \\ \text { TEA } & \text { Triethylamine } \\ \text { TFA } & \text { Trifluoroacetic acid } \\ \text { TOF } & \text { Time-of-flight }\end{array}$

ZAP-70 Zeta-chain-associated 70-kDa protein 


\section{ABSTRACT}

Antibodies have become a critical component of many diagnostic assays and are used for therapeutic purposes. Nevertheless they often fail to meet often fail to meet the strict performance demands raised in industry and in the clinic (e.g. stability, reproducibility, selectivity, affinity). These issues are especially notable for assays targeting post translational modifications (PTM) of proteins (phosphorylation, glycosylation, sulfation etc.). Antibody-based technologies suffer from problems of a more general nature associated with the analytical use of biological receptors i.e.: i) limited stability requiring cold chain logistics, ii) high production costs, iii) batch to batch variability. The above emphasizes the need for alternative robust, reproducible and low cost "binders" and assays. The aim in this thesis is to design, develop and test molecularly imprinted polymers (MIPs) which were synthesized epitope and stoichiometric imprinting approaches targeting phosphorylation as a PTM. Protein phosphorylation is one of the most common PTM, which is based on covalent attachment of phosphate group to particular amino acids. Misregulation of phosphorylation process is found related with diseases such as cancer, diabetes, and neurodegeneration. MIPs are synthesized through copolymerization of functional monomers and crosslinkers in the presence of $\mathrm{N}$ - and $\mathrm{C}$ - terminal protected templates. The key recognition element employed in developed synthetic receptors was 1,3-diaryl urea functional monomer $\mathbf{1}$. This monomer is a potent hydrogen bond donor forming strong cyclic hydrogen bonds with oxyanions. Amino acid sequence specific and side chain imprinted binders were prepared targeting phosphorylation on tyrosine (pTyr) and on histidine (pHis). pHis MIP-based approach is proposed as a solution to enrich pHis peptides in the presence of other phosphoesters such as phosphoserine (pSer) in complex mixture without pre-treatment like $\beta$-elimination. In pTyr, ZAP-70 (zeta associated $70 \mathrm{kDa}$ protein), which is prognosticator for chronic lymphocytic leukemia (CLL), and pTyr-sequence specific motif Src-SH2 domain were chosen as targets to evaluate regio- or stoichiometric selectivity performance of imprinted polymers. The synthesized polymers are used as effective enrichment tools for target phosphorylated peptides from complex mixture prior to mass spectrometry. Overall, the results demonstrate unique proteomics enrichment tools that link with personalized medicine relying on diagnostic coupled cancer treatment strategies based on kinase inhibitors. 


\section{POPULÄRVETENSKAPLIG SAMMANFATTNING}

Antikroppar har blivit en kritisk komponent i många diagnostiska analyser och används för terapeutiska ändamål. Ändå så uppfyller de ofta inte de strikta prestationskrav som ställs inom industrin och på kliniken (t.ex. stabilitet, reproducerbarhet, selektivitet, affinitet). Dessa frågor är särskilt anmärkningsvärda för analyser riktade mot post translationella modifikationer (PTM) av proteiner ( fosforyleringar, glykosyleringar, sufateringar osv). Dessutom lider antikroppsbaserade teknologier av problem av mer allmän karaktär i samband med analytisk användning av biologiska receptorer, d.v.s.: i) begränsad stabilitet som kräver kallkedjelogistik, ii) höga produktionskostnader, iii) batch-till-batch-variabilitet. Ovanstående betonar behovet av alternativa robusta, reproducerbara och billiga "bindemedel" och analyser. Syftet med denna avhandling är att designa, utveckla och testa molekylärt präglade polymerer (MIP) som syntetiserades epitop- och stökiometriska präglingsmetoder riktade mot fosforylering som en PTM. Proteinfosforylering är en av de vanligaste PTM, som är baserad på kovalent bindning av fosfatgrupp till särskilda aminosyror. Felreglering av fosforyleringsprocessen finns relaterad till sjukdomar som cancer, diabetes och neurodegeneration. MIP: er riktade mot fosforylering syntetiseras genom sampolymerisering av funktionella monomerer och tvärbindare i närvaro av $\mathrm{N}$ - och $\mathrm{C}$-terminala skyddade fosforylerade mallar. Det viktigaste igenkänningselementet som användes i utvecklade syntetiska receptorer var 1,3-diarylurea-funktionell monomer 1. Denna monomer är en kraftig vätebindningsgivare som bildar starka cykliska vätebindningar med oxianjoner såsom fosfater. Aminosyrasekvensspecifika och sidkedjiga präglade bindemedel framställdes riktade mot fosforylering på tyrosin (pTyr) och på histidin (pHis). Särskilt pHis MIP-baserat tillvägagångssätt föreslås som en lösning för att berika pHis-peptider i närvaro av andra fosfostrar, såsom fosfoserne (pSer) i komplex blandning utan förbehandling som $\beta$-eliminering. I pTyr valdes ZAP-70 (zeta-associerat $70 \mathrm{kDa}$-protein), som är prognosticator för kronisk lymfatisk leukemi (CLL) sjukdom, och pTyr-sekvensspecifikt motiv Src-SH2-domän som mål för att utvärdera region- eller stökiometrisk selektivitetsprestanda för präglat polymerer. De syntetiserade präglade polymererna används som effektiva anrikningsverktyg för målfosforylerade peptider från komplex blandning före masspektrometrianalys. Resultaten visar på unika verktyg för proteomik som länkar till personlig medicin genom diagnostikkopplade cancerbehandlingsstrategier baserade på kinashämmare. 


\section{LIST OF PUBLICATIONS AND CONTRIBUTIONS}

\section{Paper I:}

S. Shinde*, A. Incel*, M. Mansour, G. D. Olsson, I. A. Nicholls, C. Esen, J. Urraca, B. Sellergren (*shared first author), Urea-based imprinted polymer hosts with switchable anion preference. Journal of American Chemical Society, 142, 26 (2020) 1140411416.

- Contribution to paper I:

Took part in the designing of experiments and performed for the characterization of fundamental understanding of polymerization mechanism. Interpretation of the results of completed tests.

\section{Paper II:}

A. Incel, I. A. Díez, C. Wierzbicka, K. Gajoch, O. N. Jensen, B. Sellergren, Selective enrichment of histidine phosphorylated peptides using molecularly imprinted polymers. Analytical Chemistry, 98, 3 (2021) 3857-3866.

\section{- Contribution to paper II:}

Took part in the designing of experiments and performed most of the experimental part in an independent manner, interpretation the results of the tests, authored a revised draft of the manuscript. 


\section{Paper III:}

A. Incel, S. Shinde, I. A. Díez, A. Yilmaz, T. Sjoberg, M. M. Stollenwerk, O. N. Jensen, B. Sellergren, MIP-Binders for sequence specific phosphopeptide capture of ZAP-70 regulatory motifs. Manuscript.

\section{- Contribution to paper III:}

Took part in the designing of experiments and performed most of the experimental part in an independent manner, except for the synthesis of template molecules. Interpreted the results of the tests and authored the first draft of the manuscript.

\section{Paper IV:}

S. Shinde, M. Mansour, A. Incel, L. Mavliutova, C. Wierzbicka, B. Sellergren, High salt compatible oxyanion receptors by dual ion imprinting. Chemical Science, 11 (2020) 4246-4250.

\section{- Contribution to paper IV:}

Performed part of the experiments. Participated in the evaluation of results and preparation of graphic materials.

\section{Paper V:}

A. Incel, I. A. Díez, M. M. Stollenwerk, O. N. Jensen, B. Sellergren, Imprinted Src-SH2 domain mimicking: Targeting pYEEI sequence. Manuscript.

\section{- Contribution to paper V:}

Took part in the design of experiments and performed most of the experimental part, interpreted the results and wrote the first draft of the manuscript. 


\section{Other publications not included in this thesis:}

1. R. Mahajan, M. Rouhi, S. Shinde, T. Bedwell, A. Incel, L. Mavliutova, S. Piletsky, I. A. Nicholls, B. Sellergren, Highly efficient synthesis and assay of protein-imprinted nanogels by using magnetic templates. Angewandte Chemie International Edition, 58, 3 (2019) 727-730.

2. S. Shinde, J. Selvalatchmanan, A. Incel, M. Akhoundian, A. K. Bendt, F. Torta, Mesoporous polymeric microspheres with high affinity for phosphorylated biomolecules. New Journal of Chemistry, 42, 11 (2018) 8603-8608.

3. S. Shinde, M. Mansour, L. Mavliutova, A. Incel, C. Wierzbicka, H. Abdel Shafy, B. Sellergren, Oxoanion imprinted polymers combining cationic and urea binding groups: Materials for effective glyphosate capture. Submitted.

4. M. Rouhi, A. Incel, S. Shinde, Role of co-monomers in the recognition of anionic biomolecules in water: hydrogen-bonded imprinted polymeric receptor. Submitted.

5. E. Kislenko, A. Incel, K. Gawlitza, B. Sellergren, K. Rurack, Fluorescent Molecularly Imprinted Polymers for the Detection of Phosphorylated Tyrosine Epitopes of Cancer Biomarkers using Urea and Bis-Imidazolium Receptors. Submitted. 


\section{BIOCAPTURE}

This work has been carried out within the framework of a Marie Sklodowska-Curie Actions - Innovative Training Networks, BioCapture: Smart Capture Phases for Proteomics, Glycomics and Biomarker Assays.

I have been working as an Early Stage Researcher (ESR) during four years in collaboration with six other internationally renowned research teams from Denmark, Germany, Italy, Norway, Sweden, and UK, and four industrial partners from Norway, Sweden, South Africa, and UK.

The European project took place at the highly interdisciplinary intersection of the traditional disciplines of organic, polymer, and functional materials as well as analytical and clinical chemistry. This project also provided workshops, courses, conferences, summer schools, outreach activities, complementary skills trainings and interactive collaborations among the research groups and industrial partners.

The main objectives with relevance to this thesis was "to develop and validate proteomics capture phases for unstable PTMs and pY sequence motifs" carried out in the form of one work package. The following partners contributed to the work:

- Southern Denmark University (SDU), Denmark involved in testing and benchmarking of affinity materials using nanoLC-ESI-MS/MS.

- Resyn Biosciences, South Africa responsible for coupling affinity matrices to magnetic beads for automated peptide enrichments.

- Linneaus University, Sweden performing in Molecular Dynamic (MD) simulation studies.

- Proteome Science involved in assay development, benchmarking of MIPs format, comparisons and validations using clinical samples. 


\section{THESIS AT GLANCE}

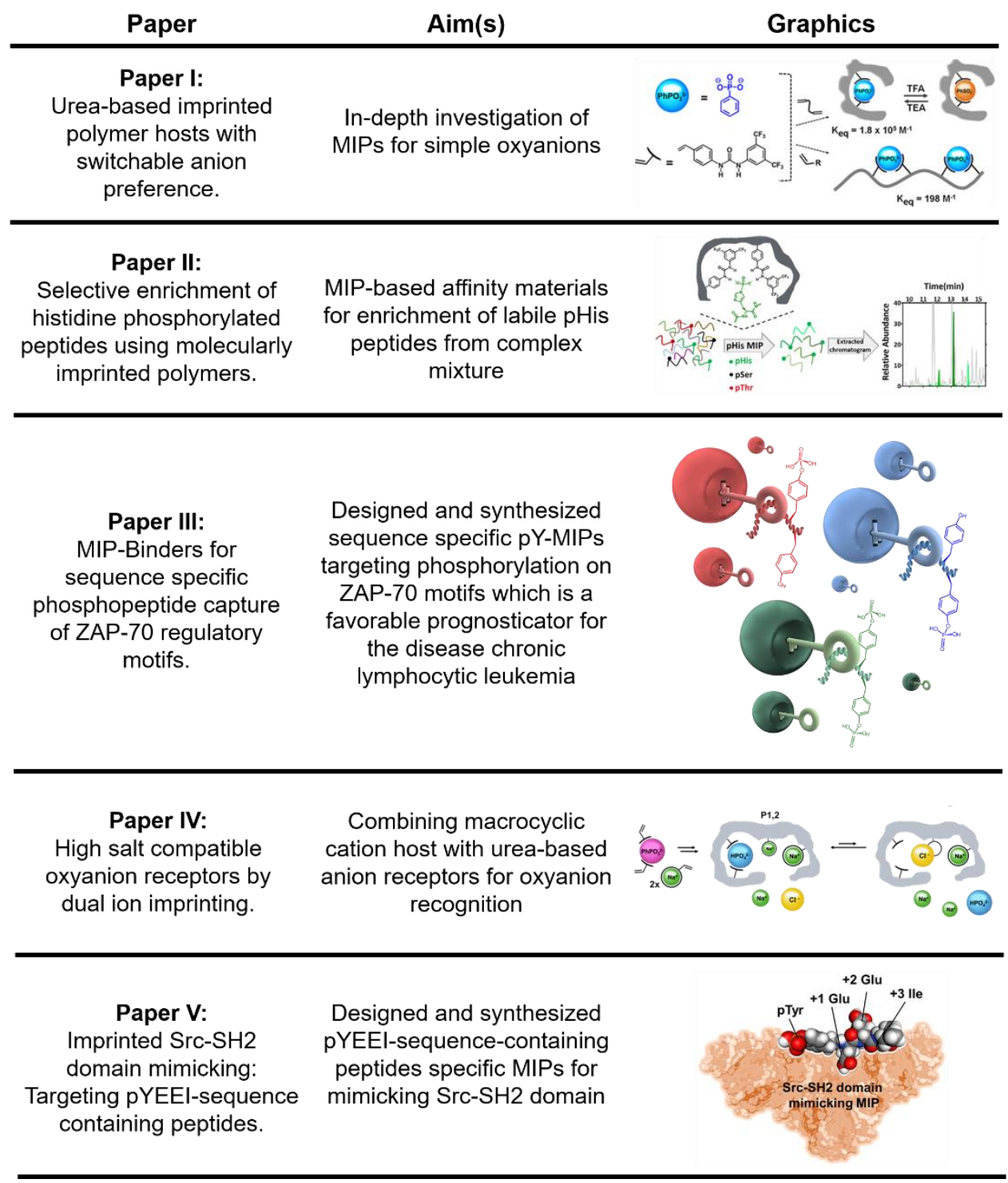




\section{MOLECULAR RECOGNITION}

\section{General Aspects}

Molecular recognition is defined as the process where two or more molecules bind to one another in a specific manner. ${ }^{1}$ This phenomenon is crucial for biological molecules such as proteins, nucleic acids, and saccharides, which play critical roles in biological reactions. Molecular recognition is of crucial importance in biological system, thus chemical research is highly motivated to mimic natural receptors.

The classical concept of molecular recognition was explained using Lock-andKey model, which was postulated by Emil Fischer in $1894 .^{2}$ In this analogy, the specific interaction between enzyme (lock) and substrate (key) suggested that only complementary geometric shapes fit exactly one to another. Then, the induced fit model was described by Daniel Koshland in $1958 .{ }^{2}$ Unlike Fischer's principle, this model suggested that enzymes are rather flexible in which the active site continuously reshapes during its interactions with substrates. Later on, the mechanism of lock-andkey associations facilitated understanding of the concept in molecular self-assembly which developed supramolecular chemistry. Supramolecular chemistry, chemistry beyond the molecule, is based on the formation of unique structural complexes between two or more species, which is mostly associated with well-defined aggregates based on noncovalent and reversible interactions. ${ }^{3}$ This concept describes the incorporation of guest molecules to the internal spaces or cavities presented in host molecules.

Host-guest (HG) chemistry is one of the most active fields of supramolecular chemistry where a range of noncovalent interactions such as $\pi$ - $\pi$ stacking interactions, electrostatic interactions, charge-transfer interactions, hydrogen bonding, and Van der Waals force can be employed to assemble host networks for binding of guest molecules. ${ }^{3}$ Since the specificity is the most pivotal for molecular recognition, HG interaction to occur, host molecule must possess the appropriate binding sites for the guest molecule to bind to where host-binding sites converge and guest-binding sites diverge in the complex. These specific spatial arrangements can arise from different factors such as the complementarities of host and guest binding sites, the pre-organization of host conformation and cooperativity of binding groups related with guest structure. 
Supramolecular chemistry comprising HG chemistry or molecular recognition chemistry, has been extensively used in order to obtain molecules with ability to recognize specific guest species. ${ }^{4,5}$ Starting from mid-to-late 1960 s, the use of supramolecular chemistry became one of the fast-growing fields particularly in the development of macrocyclic ligands such as crown ethers or cyclic ethers, cryptands, spherands, cyclodextrins (Figure 1) for metal cations. ${ }^{6,7}$

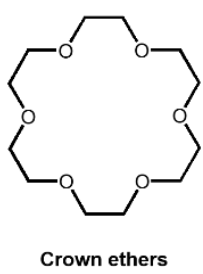

Crown ethers

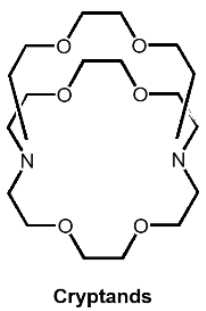

Cryptands

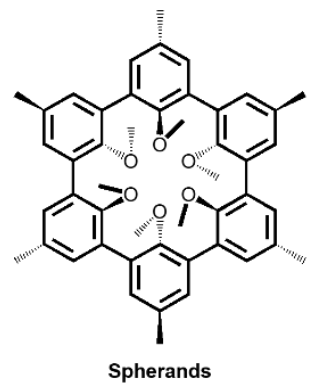

Spherands

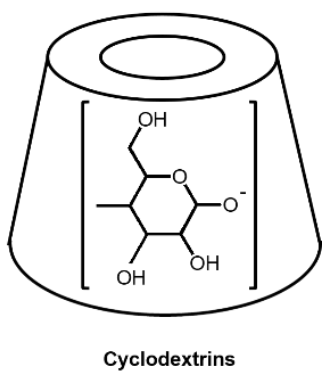

Cyclodextrins

Figure 1: Some examples of macrocyclic host ligands. [18]-crown-6, [2.2.1]cryptand and a single glucopyranose unit are given for crown ether, cryptand, cyclodextrin structures, respectively.

Crown ethers are known as the first artificial host molecules discovered by Charles Pederson in $1967 .^{8}$ These cyclic compounds are constructed with ethylene bridges separated by oxygen atoms. In 1969, Jean-Marie Lehn introduced azacrowns where some of oxygen atoms in crown ether replaced with nitrogen functionalities. ${ }^{8}$ The versatility of azacrowns macrocycles has subsequently made it possible to syntheize three-dimensional analogues of crown ethers by introducing bridges within the crown ether ring, so called cryptands. In 1973, Donald Cram introduced rigid and bowl-shaped macrocyclic structure called spherands offering an elegant and precise means of determining the influence of pre-organization on host molecules ligand binding. ${ }^{8}$ Another example of extensively studied host are the naturally occurring cyclic structures called cyclodextrins. These important molecules are cyclic oligosaccharides consisting of a macrocyclic ring of glucose subunits linked by $\alpha-1,4$ glycosidic bonds. ${ }^{8}$ Cyclodextrins are coupled with primary and secondary hydroxyl groups providing water solubility and a central hydrophobic cavity or cage for the complexation with guest species. Among these macrocyclic ligands, crown ethers and cryptands have been extensively used due to their structural features which allow specific complexation with a number of cationic species. 
To understand the relationship between ionic diameters of alkali metal cations and the cavity size of particular crown ethers, some examples are given in Figure $2 .{ }^{9}$ Not only simple crown ethers, but also their three-dimensional analogs, cryptands and spherands, have been studied with respect to cation-cavity size match. This showed that the more pre-organized hosts have better ability to discriminate the guest cation.

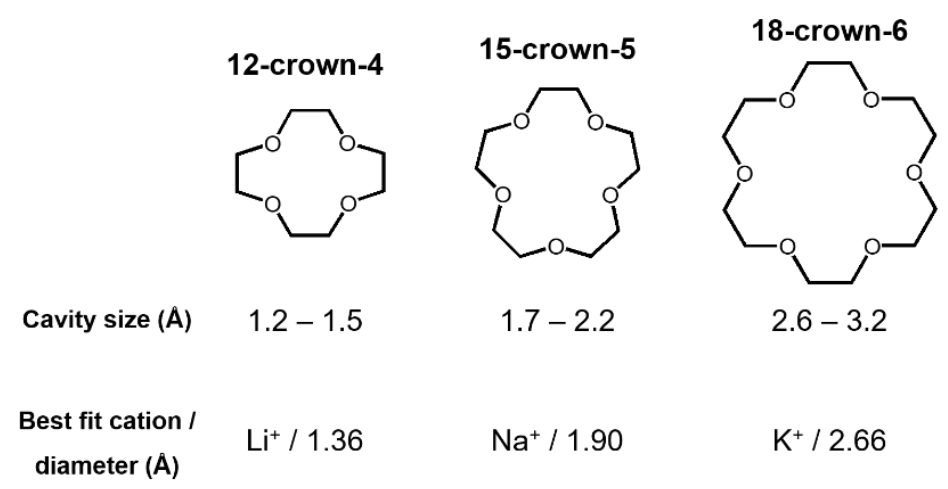

Figure 2: Some of crown ethers with their cavity sizes and the best-fit cations with their ionic diameters. Adopted from Cragg et al. ${ }^{9}$ with permission from Wiley.

Since Charles Pederson's discovery of crown ethers complexed with alkali metals, the field has experienced a very strong growth with numerous reports on the design and development of high affinity materials for particular targets. Later in 1987, Donald J. Cram, Jean-Marie Lehn, and Charles J. Pederson was awarded the Nobel Prize in Chemistry for the development and use of molecules with structure-specific interactions of high selectivity and this award has drastically increased the significance of supramolecular chemistry. ${ }^{8}$ The versatility of supramolecular chemistry and its subdiscipline involving assembling of molecules to form complex assemblies has led to diverse fields of research comparing the design of synthetic receptors and their applications in sensors, catalysis, nanotechnology, medicine, biomimetics, and molecular devices. In 2016, Jean-Pierre Sauvage, Sir J. Fraser Stoddart, and Bernard L. Feringa were honored with Nobel Prize in the field of supramolecular chemistry for their contributions to the design and synthesis of molecular machines. ${ }^{10}$ Their essential work was on the realms of mechanically bonded molecules and sterically crowded olefins, which further improved the design of more complex artificial structures. Over the last three decades, their remarkable accomplishments and discoveries have contributed significantly towards HG chemistry particularly mimicking and understanding biological systems. 


\section{Molecularly Imprinted Polymers (MIPs)}

\section{General Concept of MIPs}

Sensitivity and specificity are the key factors for ultimate form of sensing where the ability of a host to bind its particular target in molecular recognition. In biological machinery, how natural receptors such as antibodies are able to be produced via immune response in the presence of any foreign or unknown guests in our body, such recognition can be adopted for the creation of synthetic receptors to mimic the natural systems. For instance, molecular recognition elements with selective, sensitive, and robust in combination with HG and modern chemistry can offer manufactured synthetic receptors for detection of target of interest. These manmade binders might also overcome disadvantages of their biological analogues such as fragility with respect to environment conditions and high-cost. ${ }^{11}$

Molecularly imprinted polymers (MIPs) are a new generation of robust synthetic receptors. They are also called as plastic antibodies, which offer a new generic and cost-effective platform for synthesis of specific molecular affinity materials into polymer matrices. ${ }^{12-16}$ This imprinting strategy is based on process of prearrangement of recognition sites around target molecules or their analogues, template, using functional molecules to form a scaffold around within a matrix forming an imprint, which present binding sites that are complementary in size, shape, and functionality for an affinity towards template or template substructure larger molecules (Figure 3a). Control polymers called non-imprinted polymers (NIPs) which are synthesized by omitting template molecule in the synthesis of polymer network (Figure 3b).

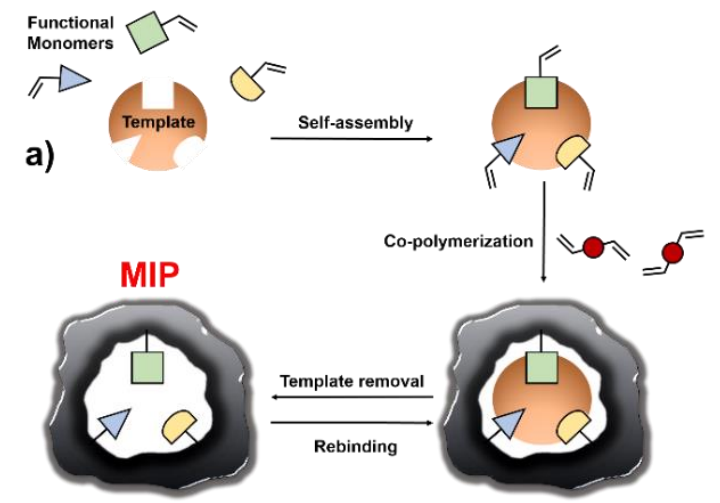

b)

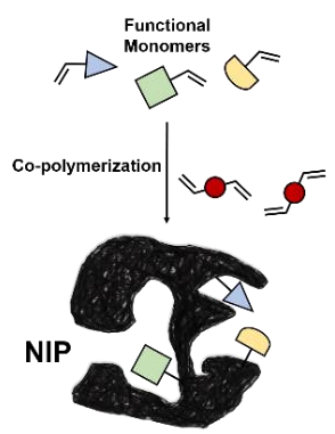

Figure 3: The schematic explanation of synthesis of Molecularly Imprinted (MIP) and Non-imprinted (NIP) polymers. 
Imprinting approach has long story starting from the very first paper published by Polyakov in $1931 .{ }^{11}$ Polyakov studied on silica polymerization and how silica pore structures differentiate using three different solvents, which are benzene, toluene, and xylene. Polyakov's investigations showed a positive correlation for the adsorption of particular solvent at some extent to silica gels using acidic or basic additives. This was the first time where this kind of scientific study applied in the explanation of how the presence of template molecule might alter the behavior of end material in the adsorption process. After particular studies completed by Linus Pauling on understanding of protein structure, functions, and mostly, the selective and instructional theories on antigen selectivity of antibodies, the idea of bio-imprinting inspired Frank Dickey in the 1940s where he described the term, pattern molecules..$^{11}$ In the study, he synthesized silica matrices in the presence of different dye molecules, which were subsequently removed after the synthesis of sorption gels. Then he compared the relative adsorption of dyes to the corresponding and non-corresponding silica materials; moreover, the one synthesized in the absence of dye was used as control. These adsorbents showed pronounced selectivity towards the corresponding dye molecule, which was used in the synthesis of particular one. This study played an important role to understand the ligand-selective recognition using imprinting approach. Moreover, concerning of template removal procedure, effects of proper extraction were emphasized in following studies where various agents were subjected for template removal to minimize the defects in imprinted materials with enhancing binding selectivity. In 1955, R. G. Haldeman and P. H. Emmett were the first researcher who used imprints, have restudied of Dickey's silica samples and reported on that lower binding and insignificant selectivity due to aging of silica particles in time. ${ }^{11}$ Dickey also approved this inconsistency and more studies have brought new findings such as the electrostatic interactions were found as driving for the recognition process for this particular case, which was also supported by others. These studies also helped to understand the relation between the adsorption of target by binder and the dielectric constant of binding solvent. Later in 1959, the association mechanism of imprints were studied by John L. Morrison to understand how adsorption of target can be enhanced where he concluded that complete removal of template removal by acidic washing can increase target molecules accessibility to created cavities. A. Waksmundzki studied on the importance of template molecules on surface area and porosity of synthesized imprints. Molecularly imprinted silica materials became so popular and used in many practical applications such as separation and catalysis. ${ }^{11}$ The limitation on sol-gel imprinted silica particles such as lost target memory in time and non-reproducibility caused temporary decline on this research area. 
Considering the potential of imprinting and principal disadvantages of silica method, a much better possibility to prepare more stable, reproducible, and controllable materials using imprinting approach was needed. Therefore, the molecular imprinting in synthetic organic polymers was introduced in $1972 .{ }^{17}$ The first promising imprinting in synthetic polymers was done by G. Wulff and A. Sarhan. This study is based on use of covalent approach to create imprinted polymer for the recognition of D-glyceric acid that used as template in the imprinting process together with a boronic ester and an amide as functional monomers. This achievement was later on significantly broadened the molecular imprinting concept by K. Mosbach when he introduced noncovalent approach. The papers entitled "Imprinting of amino acids derivatives in macro porous polymers" by K. Mosbach and B. Sellergren in $1984{ }^{18}$ and "Enzyme-analogue built polymers" by G. Wulff in $1985^{19}$ had positive impact on imprinting concept and caused exponential increase in interest for molecularly imprinted polymers by many researchers. From 1980s to now, MIPs extensively employed in various applications such as detection, separation, sensing, enrichment and enzyme/antibody mimics.

\section{Fundamental Aspects of MIPs}

\section{Polymerization}

The formation and structure of polymer network in the field of molecular imprinting is based on polymerization mechanism. Polymerization is the process of creating larger macromolecules or higher molecular weight materials using relatively smaller molecules or repeating units called monomers. In general, polymerization reactions can be divided into two main groups: chain-reaction (addition) and step-reaction (condensation) polymerization (Figure 4).

(a)

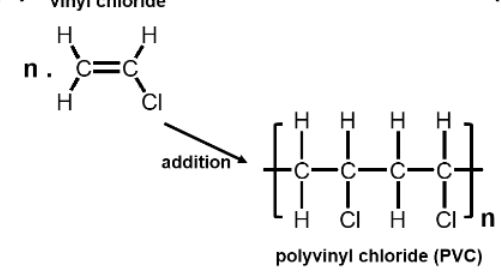

(b)

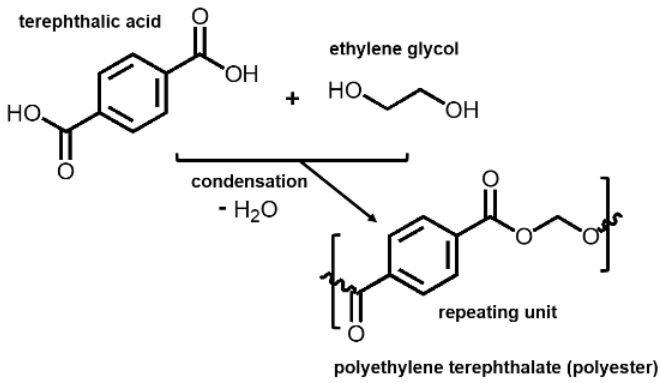

Figure 4: Examples for chain-reaction polymerization (a) where the formation of polyvinyl chloride from n number of vinyl chloride monomers and step-reaction polymerization $(b)$ where the formation of polyester by repeating unit of formed ester complex showed. 
These two types of polymerization can differ in terms of degree of polymerization, conversion rate, and polymerization rate during process, or requiring additional reagents for reaction. ${ }^{20}$ Chain-reaction (addition) polymerization has also three subgroups: free radical, ionic and complex coordination polymerization. Majority of synthesized MIPs are based on free radical copolymerization of vinyl monomers with an excess amount of crosslinking agent in the presence of porogenic solvent.

\section{Free radical polymerization}

Free radical polymerization is based on a mechanism where polymer molecules grow by the repetitive addition of monomer to a terminal free-radical reactive site. The term free radical stands for highly chemically reactive species, which can be activated by thermal or photochemical ways. Initiators are the source of free radicals..$^{20}$ Peroxide, and azo- initiators are commonly used examples. Free radical polymerization has three main mechanistic steps: initiation, propagation, and termination (Figure 5). In initiation step, the process starts with the formation of radicals using proper initiator followed by that the generated free radicals attack to the $\pi$-bond of the monomers where the alkene undergo breakage of single bond which creates a new and larger free radicals. In the propagation, the generated larger radicals react with another monomers and the reaction follows as chain propagating process. In the final step, termination occurs when two active chain ends interact with each other either combination (two grown chain form a stable molecule) or disproportionation (when radical transfer hydrogen to make two stable molecules) ways.
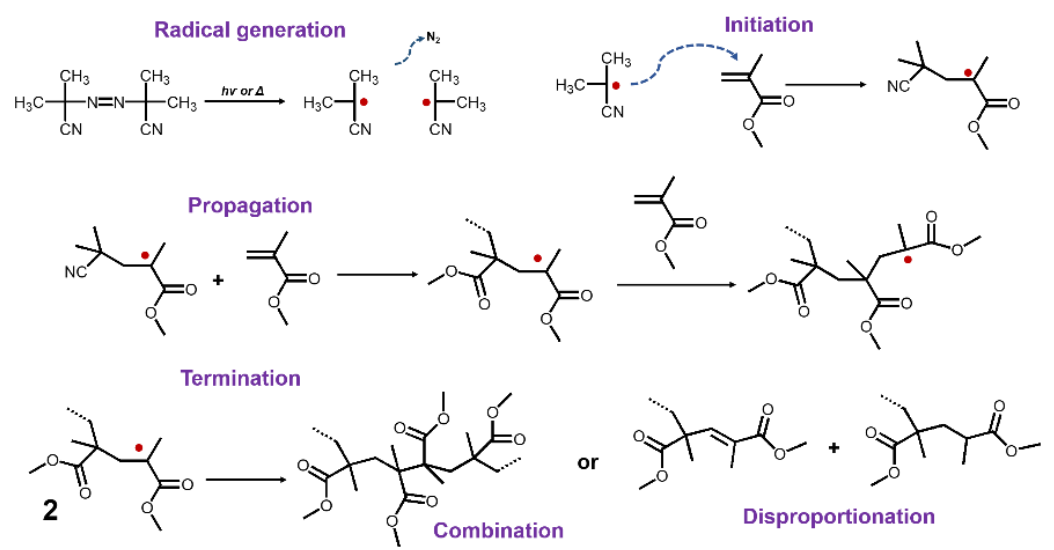

Figure 5: Free radical polymerization steps: radical generation, initiation, propagation and termination. Polymerization of methyl methacrylate (MMA) with AIBN initiator is shown as example. 


\section{Copolymerization}

Polymerization process where two or more different type of monomers involved called as copolymerization. The vast majority of identified imprinted polymers are copolymers particularly where co-polymerization between functional monomer or monovinyl monomers and crosslinking agent or divinyl monomers used. Copolymers can be in three different microstructures: random or statistical, alternating, and block, depending on monomer's relative reactivity in the synthesis. ${ }^{20}$ In random or statistical copolymers, the addition of each monomer can be ideally or truly random such as AA-B-B-B-B-A-B-A-A-B-B-A-B; in alternating, after each A monomer follows with $\mathrm{B}$ such as A-B-A-B-A-B-A-B-A-B-A-B-A-B; and when individual monomer is more reactive to its own unit, it has tendency to generate blockings such as A-A-A-A-A-AA-B-B-B-B-B-B-B.

Kinetic of copolymerization mechanism is the key factor on which type of copolymer is formed. The particular reactivity ratio of generated monomeric radicals $\left(\mathrm{r}_{1}\right.$ $=\mathrm{k}_{11} / \mathrm{k}_{12}$ and $\left.\mathrm{r}_{2}=\mathrm{k}_{22} / \mathrm{k}_{21}\right)$ depends on rate constants for self- $\left(\mathrm{k}_{11}\right.$ or $\left.\mathrm{k}_{22}\right)$ or cross$\left(\mathrm{k}_{12}\right.$ or $\left.\mathrm{k}_{21}\right)$ propagating reactions. If self-propagating reactions are more favorable than cross-propagating ones $\left(\mathrm{k}_{11}>\mathrm{k}_{12}\right.$ and $\left.\mathrm{k}_{22}>\mathrm{k}_{21}\right)$, the mechanism has tendency to form block- or homo- copolymers; in contrary alternating copolymers form. ${ }^{20}$ In ideal case, when $r_{1}$ and $r_{2}$ equal to 1 , monomers incorporate randomly in polymer network. The formation of different type of copolymers may affect stereochemistry which can influence physical and chemical properties of polymers. The pendant groups on a polymer chain arranged in the backbone can diverse tacticity of polymers and the changes in tacticity might cause effects on physical properties of polymers such as having more amorphous or more crystalline structures.

Such MIPs synthesized by free radical copolymerization in the presence of excess number of crosslinking agents called highly crosslinked polymers. Since they are excessively crosslinked polymer networks, the flexibility of polymer chains are lower and this was hypothesized as better for shape related recognition. Considering the comparison of linear polymers (only monovinyl monomer introduced) and copolymers, the effect of crosslinking process on tacticity and regularity of chain formation is important to assess. ${ }^{11}$ If the configurations are the same in polymer chain, they are called isotatic, if it is alternating called syndiotactic and if there is no repeating configuration, they are atactic. The tacticity of polymer chain might affect the recognition of enantiomeric molecules; however, there is no clear explanation on what extent effecting on recognition properties. 


\section{Kinetics}

Kinetic and structural properties of template-tailored synthesized polymers using copolymerization of vinyl and divinyl monomers can vary. Earlier, the rate constants related to self- or cross- propagation reactions were mentioned. Thermodynamically initiated high crosslinked copolymerization mechanism is kinetically considered as different from the formation of linear polymers. The presence of crosslinking agent and its possible interactions with vinyl monomers and template molecules definitely affect the formation of a specific geometry of recognition cavities or regions in imprinted complex. ${ }^{11,21}$ In principle, copolymerization of two or more different monomers, their rate constants, their chemical properties of polymerizable units, and their intramolecular interaction cycles contribute physical formation of polymer networks, and regulate principles in affinity materials. A variety of interacting species in polymerization process such as temple-monomer complexation, monomer-monomer interactions, monomer-crosslinking interactions, crosslinking-template and/or crosslinking effect on template-monomer assemblies influence kinetic parameters in the polymerization.

The rate of polymerization strongly depends on the reactions in initiation, propagation, and termination steps that were earlier mentioned. How monomeric radicals generated, how the pendant groups react with each other-it can start forming polymer chain or some unreacted monomers can be trapped- how presence of excess crosslinking agent affect polymer viscosity, how conversion of system from monomers to polymer change by the changing of polymerization environment-since when polymer viscosity increases the gelation might accelerate termination process decreasing the rate of initiation - and how these all parameters could affect the recognition pockets that aimed to be created in the polymer network must be taken into account. ${ }^{11}$

In terms of understanding the fundamentals in kinetic of polymerization, some useful information can be obtained investigating primary, secondary, and tertiary structures occurring during polymerization. Primary structures is mostly related wit particular monomers used in the polymerization and estimation their relative reactivity. At some extent, the interaction among template, monomer and crosslinking agents, their solubility in the choice of porogen, temperature of polymerization can be studied to make some predictions before starting polymerization process.

Since the interaction between template and functional monomer is essentially the key for the formation of proper recognition pocket, the effect of crosslinking agent right after addition, after starting polymerization and how it would affect the interaction between template and monomer can be considered as secondary structures. 
When formation of polymer chain starts, additional strain introduced in polymerization should not affect the three-dimensional network. The formation of multidentate interactions upon polymerization of functional monomers is considerable important for the mechanism of specific site formation. Tertiary structure is about the formation of three-dimensional polymer network and its physical properties such as porosity, target accessibility, size of particles, inner size of scaffold, surface area, swelling characteristic, etc. The choice of solvent has significant effect on the resulting polymer structure since it would affect swelling capacity of cavities, the distance between functional groups presented in polymer network and polymer morphology.

Considering diversities in the formation of MIPs might help to eliminate possible heterogeneities or polyclonality and non-specific binding sites (Figure 6) might form in the imprinted materials. ${ }^{22}$ Figure 6 represents the formation of possible binding sites based on different accessibility, integrity and stability. For instance, there are macro- or meso- porous structure ( $\mathrm{D}$ and $\mathrm{H}$ ) where accessibility of template in rebinding will be higher compared to micro-pores ( $\mathrm{A}$ and $\mathrm{C}$ ) where the diffusion will be slower. Comparing the binding sites represented macro- or meso- pores to micropores, microporous might show higher for a given volume. There can be undesirable reactions such as non-complexed monomer in the pre-polymerization (E and $\mathrm{G}$ ), lower affinity binding sites due to the imprecise rearrangement of functional monomers around template (B, D, E and $\mathrm{H}$ ), or some collapsed binding sites after template removal (B).

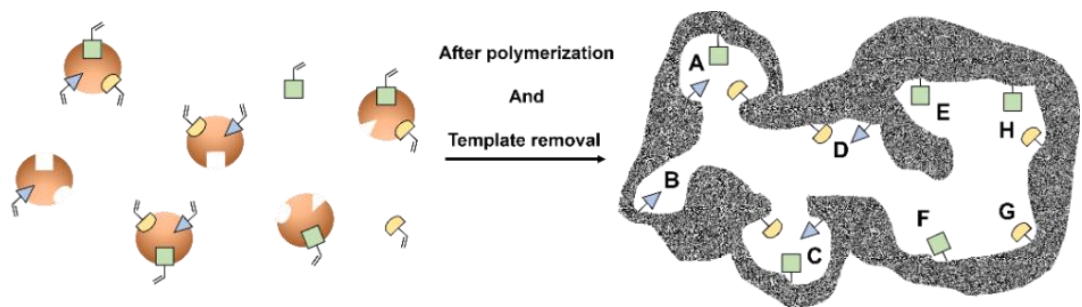

Figure 6: The undesired reactions formed during the polymerization process (left) and its possible effects on heterogeneity in the polymer network after polymerization and template removal (right).

However, not these all polyclonality reflect the full image of one particular imprinted polymer since the diversity could be even higher. For this reason, in order to understand the particular imprinted polymer synthesized for target of interest should be precisely analyzed and characterized before and after polymerization. 


\section{Characterization of MIPs}

Nuclear Magnetic Resonance, NMR is used to study the structure of compounds and their interactions with each other. The titration experiment carried out between monomers and templates can result chemical shifts upon the formation of HG complexes. The data can be fitted binding models to get information about the association constant and stoichiometry between reagents.

Infrared Spectroscopy is used to obtain a number of useful band information about the structure and formation of polymer chains. The monomer reactivity ratio upon conversion can be monitored since the vibration of $-\mathrm{C}=\mathrm{C}-$ vinyl carbons has characteristic absorption peaks between $1600-1641 \mathrm{~cm}^{-1}$.

Thermal Gravimetric Analysis, TGA can give rough measurement about the yield of polymerization comparing particular polymer sample before and after template removal.

Differential Scanning Calorimetry, DSC can give information about the thermal behavior of polymers. For instance, using different crosslinking agents might give different glass transition temperature and removal of template from certain amount of polymer can lead some changes in the melting transition.

Nitrogen Adsorption/Desorption Analysis is used to evaluate Brunauer-EmmettTeller (BET) surface area, total pore volume, average pore diameter, and pore size distribution that helps to get information about pore structure of polymers.

Microscopy such as scanning electron microscopy (SEM) is used to examine imprinted polymers morphologically such as if it has irregular structure or not.

Elemental Analysis is one of the traditional methods used to obtain information about the elemental composition of any material. Quantitative analysis of compounds can be done detecting such as hydrogen, oxygen, nitrogen, halogens, sulphur and phosphorus and compared with the theoretical composition for estimating polymerization yield. 


\section{Thermodynamic Principles underlying MIPs formulation}

The development of an imprinted polymer, which has great complementary and sufficient selectivity towards its particular target, is extensively related with the strengths between template-monomer, monomer-monomer, template-solvent, and monomersolvent presented in the pre-polymerization mixture. Since vast majorities of variables play a critical role to utilize the formation of desired end material, fundamentals behind the interaction of a host and a guest and why or how they do or do not interact with each other; the physical basis of molecular recognition can be thermodynamically explained. In order to define the physical terms that involve in recognition events was thermodynamically expressed by Williams equation (1) which can help to understand the principal contributions of favorable and unfavorable interactions in complex formation..$^{23,24}$

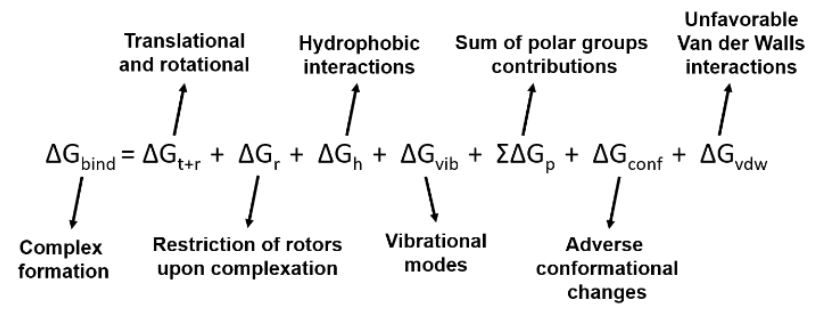

When a pre-polymerization mixture is prepared prior to synthesis of imprinted polymer, the formation of template-functional monomers and the relative strength of interactions depending on noncovalent or covalent imprinting approach play significant role for the number of binding sites and the degree of polyclonality which can be determined by $\Delta \mathrm{G}_{\mathrm{bind}}$. In addition to that, the binding characteristics of a polymer can be examined in terms of physical basis for MIP-ligand interaction. For the recognition of ligand by imprinted materials, $\Delta \mathrm{G}_{\mathrm{conf}}$ and $\Delta \mathrm{G}_{\mathrm{vdw}}$ enthalpic energies can be assumed as non-zero values since the recognition pockets formed in polymers should not possess any conformational strain and adverse interactions. The changes in $\Delta \mathrm{G}_{t+\mathrm{r}}$ should bring energetic advantages while monomers interacting via decreasing number of freedom upon complexation. $\Delta \mathrm{G}_{\mathrm{r}}$ can be minimized due to decreasing moments of inertia, the more rigid templates presented, the less heterogeneity formed. $\Sigma \Delta \mathrm{G}_{\mathrm{p}}$ is about how the electrostatic interactions related with solvent polarity to favor interactions. Maximizing $\Sigma \Delta \mathrm{G}_{\mathrm{p}}$ leads to form more stable complex with better site fidelity. $\Delta \mathrm{G}_{\mathrm{h}}$ is an assembling more favorable interactions arises upon the removal of hydrophobic surfaces. Overall, to produce an imprinted polymer with high-fidelity sites, greater affinity and selectivity, these factors can be considered. ${ }^{24}$ 


\section{Noncovalent Imprinting}

Noncovalent approach in imprinting has been dominantly used to facilitate molecular recognition using these tailor-made affinity materials. ${ }^{25-30}$ Noncovalent imprinting is mainly based on generating hydrogen bonds, ion-pairs, dipole-dipole interactions and van der Walls forces amongst functional monomers and template molecules. In the imprinting process, it is assumed that first complexation occurs between functional monomer and template and then polymer chains grows around this prearranged structure to make more robust end material. Noncovalent approach has its own strengths for imprinting such as its relative simplicity and range of chemical functionalities, translating monomer functionality regarding to template of interest, being dynamically reversed and much faster rebinding kinetics compared to covalent approach. On the other hand, this approach has some major drawbacks like heterogeneity of receptor sites that can yield formation of high and low affinity binding sites, and having low percent of high-affinity pockets compared to template amount used in the prepolymerization mixture due to inadequate rigidity.

\section{Reagents for MIPs synthesis}

Considering the previous sections on copolymerization, kinetics and thermodynamic, the prearrangement of template-monomer and follow-up polymerization process have more dynamic environment and unstable mechanisms. For this reason, the complexity of any particular system should be understood by choice of template, functional monomer (s), crosslinking agent and its density or level, porogenic solvent, and initiator type. These all reagents are important which need to be taken into consideration in detail prior to synthesis of imprinted polymers for particular target.

\section{Template}

Template or target-like molecule is the key reagent for preparation of imprinted polymers. The template, which is going to be used in pre-polymerization mixture, can be target itself or it can be target specific motif such as an epitope. If it is a custom designed template, the specific target structural features of template molecule should fulfill the criteria to make it suitable for imprinting. These templates should be resistance for polymerization conditions, able to solubilize in the choice of solvent, stable in terms of degradation and conformation, and the most important is the ability of forming desired complexation with monomers. Amino acids, proteins, pollutants, drugs and many clinically important molecules have been successfully imprinted..$^{31-33}$ 


\section{Functional Monomers}

Since molecular recognition provided by imprinting technology is about the strength and positioning of the template-monomer complexation, the choice of functional monomer or monomers composition play a critical role to create highly specific recognition pockets in imprinted material. Not only the structural features of template molecules, but also stabilization of monomer-template assemblies, template size and shape, monomer-template conformational rigidity should be taken into consideration while choosing functional monomer(s). Some of acidic, basic, and neutral commercially available functional monomers were shown below.

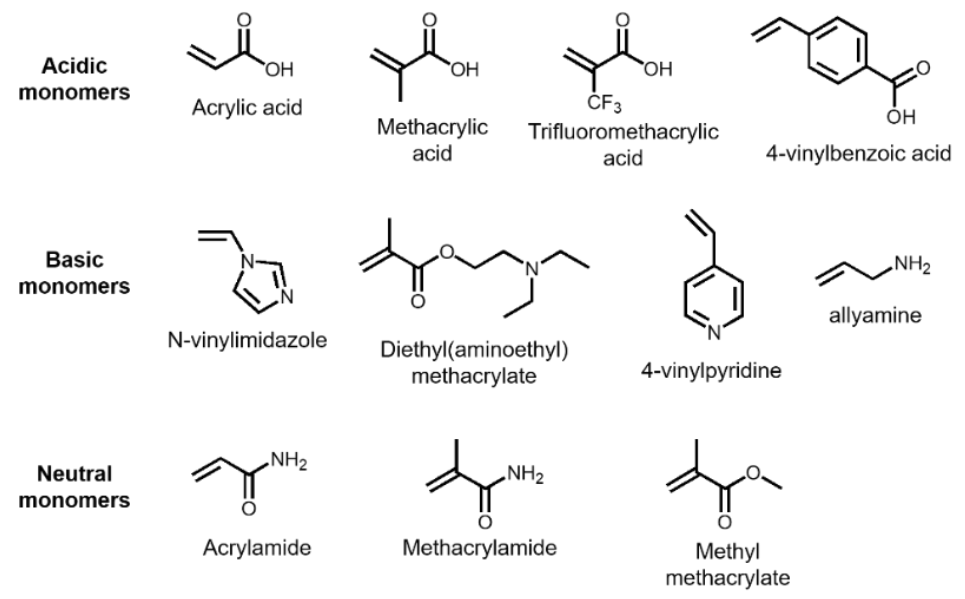

Due to their dual hydrogen bond donor and acceptor property, carboxylic acid-derivative monomers have attracted to be used in imprinting process for the basic templates. ${ }^{15,34}$ Especially, methacrylic acid (MAA) have been extensively carried out in imprinting process since bulk methyl group presents additional advantage such as restriction on rotation and conformational flexibility which can fulfill shape-selective elements of binding pockets. Introducing trifluoromethyl group in the acrylic acid bone can enhance binding selectivity due to increased acidity in the monomer. ${ }^{35} \mathrm{Con}$ cerning about acidic templates, basic monomers were particularly well suited for imprinting; the vinyl pyridines, vinyl imidazole, notably amidines have been developed for imprinting use. ${ }^{36}$ Many experiments were carefully studied on finding optimal structure motifs provided by monomers to complement target molecule functionalities which further leads synthesis of new custom-made monomers such as 2,6-bisacrylamidopyridine for cyclic imide, nucleotide base adenine-based monomer for carboxylic acids, and a polymerizable bis-urea monomers for amino acids..$^{37-40}$ 


\section{Crosslinking agent:}

The use of crosslinking agents in imprinting has many functions such as controlling morphology, stabilizing binding pockets, and providing mechanical stability. ${ }^{41-44}$ Various crosslinking agents that are commercially available and widely used in imprinting were shown below.

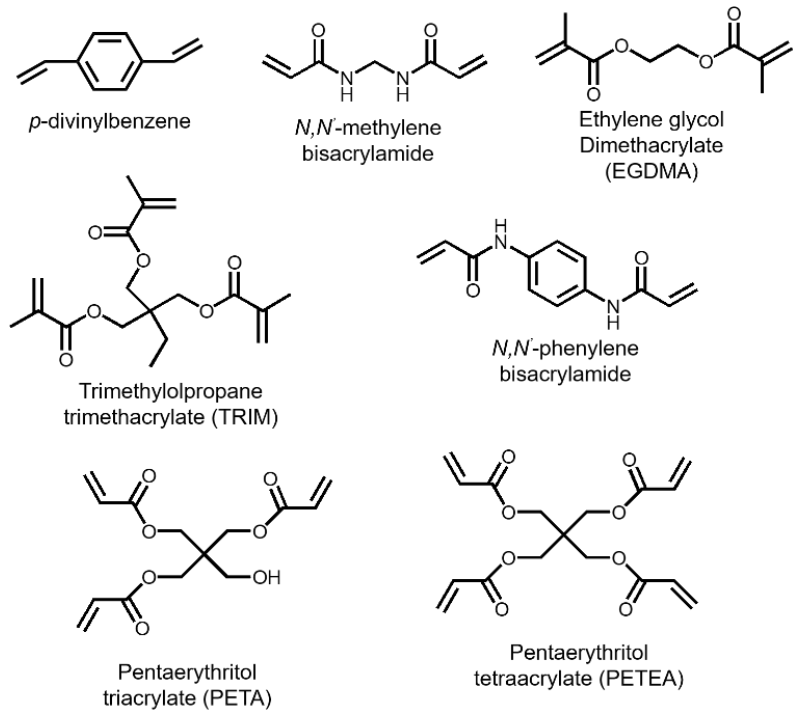

The choice of crosslinking agent is significantly depending on the choice of monomer since polymerizable units should have similar functionality or reactivity to provide equal distributions in polymer matrix. Methacrylate-based crosslinkers are the most commonly used ones in imprinting due to their mechanical and thermal stability, good solubility and wettability in pre-polymerization mixture and compatibility with most rebinding media of ligands, and their rapid mass transfer ability. If strong and stoichiometric complexation between template and functional monomer is provided in prepolymerization mixture, the effect of crosslinking agent on template should be negligible. However, crosslinking agent has high impact on physical properties of polymers. In this manner, crosslinking density play a critical role on polymer morphology, surface area, porosity, and consequently template rebinding capacity. Crosslinking percentage is usually higher than $70 \%$ of the total monomer composition to generate robust end materials and to provide sufficient rebinding of ligand. Amount of crosslinking agent can be optimized regarding to target of interest and its end-use such aqueous-based rebinding environment might require less amount of crosslinking to enhance swelling capacity. 


\section{Porogenic solvents:}

The simple role of porogenic solvent used in imprinting is to bring all reagents used in polymerization into one phase. Another and the most important one is to help creating porous morphology in the polymer network, which enhances accessibility for rebinding mechanism. ${ }^{11,30,45}$ The choice of porogenic solvent is highly depending on template, functional monomer(s), and crosslinking agent since solvent should readily solubilize all reagents without interfering their interactions, and promote the formation of template-monomer complexation during polymerization process. Therefore, not only solubility of reagents is important but also polarity of solvent plays a substantial role. For this reason, non-polar and less polar organic solvents, such as toluene, acetonitrile and chloroform are often carried out as porogenic solvent especially in noncovalent imprinting approach. Considering the interactions in noncovalent imprinting, typically a combination of electrostatic (hydrogen bonding, ionic interactions) and hydrophobic interactions ( $\pi-\pi$-stacking, van-der-Waals interactions), good imprinting efficiency and affinity towards target is achieved when imprinting is done offering low hydrogen bond donor and acceptor an aprotic solvent with low dielectric constant. In terms of polymer properties prepared in good or bad solvent, the growing polymer chains in polymerization process can be affected. Having poor solvent can cause formation of open pore structure introducing more particle mechanism, which leads phase separation when microstructures precipitated. In contrary, homogeneous network provided by good solvent can create intermolecular crosslinking where high swelling ability can be observed compared to poor solvent.

\section{Initiators:}

The most common preparation of imprinted polymers is based on free radical polymerization using thermally or photo-chemically initiators. ${ }^{30}$ Azo compounds, particularly azobisisobutyronitrile (AIBN) and azobisdimethylvaleronitrile (ABDV) are widely used and the chemical structures were shown below.
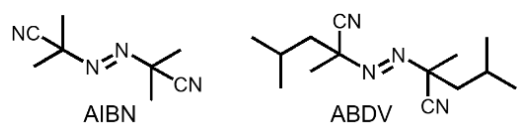

These initiators can decompose at the temperatures of $50{ }^{\circ} \mathrm{C}-70{ }^{\circ} \mathrm{C}$. To initiate the polymerization, the mixture has to be degassed by bubbling inert gas such as argon or nitrogen to remove dissolved oxygen which can inhibit the polymerization. 


\section{PROTEIN PHOSPHORYLATION}

\section{Proteomics and Post-Translational Modifications (PTMs)}

The central dogma of molecular biology is described as follow: The two major types of nucleic acids are Deoxyribonucleic acid (DNA) and RNA (ribonucleic acid). Nucleic acids are linear polymers of nucleotides strung together in a genetically determined order that is critical to their role to store all the generic information that an organism needs to develop, function, and reproduce. ${ }^{46}$ DNA and RNA differ in their chemistry and in their role in the cell. While RNA is mainly single stranded, DNA forms a double-stranded helix based on complementary base paring. DNA serves primarily as the repository of genetic information, whereas RNA molecules, mRNA, tRNA, and rRNA function mainly in expression of the information in DNA by their involvement in protein synthesis. After RNA, molecules are synthesized in the nucleus they are translocated to the cytoplasm. Prior to translocation, mRNA is used in transcription of the template strand of DNA. Thereafter ribosomes, mRNA and tRNA together perform protein synthesis in the cytoplasm of the cell by translating the nucleotide sequence into the corresponding amino acid sequence with the help of the genetic code. Every three mRNA bases are converted to one particular amino acid and form unfolded, long chain of amino acids, also known primary structure sequence. Before newly synthesized polypeptides can function properly, they must fold into the correct three-dimensional shape. The polypeptide chain fold into two main structures, which are $\alpha$-helixes and $\beta$-sheets and form secondary protein structure. These multiple structures create tertiary protein structures where three-dimensional folding occur due to chain interactions. If proteins consists of more than one amino acid chain, quaternary protein structures form into multimeric proteins such as hemoglobin.

Proteomics is an interdisciplinary domain which generally address to the largescale study of proteins that enables the identification of proteomes, but often denote specifically to protein purification and mass spectrometry. ${ }^{47,48}$ Newly synthesized polypeptide chains often require chemical modification before they can function properly, so called post-translational modifications (PTM). Phosphorylation, glycosylation, methylation, protein splicing are crucial examples amongst other 400 different types of PTM. ${ }^{49}$ The chemical alterations in the formation of polypeptide chains 
extend the diversity in protein structure and their functions. Protein-coding genes frequently give rise to multiple distinct protein species, so called proteoforms that have a unique primary amino acid sequence and localized PTMs. This can also result in partition into different protein complexes or show functional differences. Thus, PTM processes make protein structure more complex. The $\sim 20,000$ coding genes are currently estimated to generate more than a million different proteoforms folding in different ways with multiple PTMs. These proteoforms can differ between individual cells, tissues, and disease phenotypes. ${ }^{50,51}$

\section{Phosphorylation as a PTM}

Phosphorylation is one of the most widespread type of PTMs. This process is based on the reversible mechanism of kinases and phosphatases that protein kinases catalyze the addition of $\gamma$-phosphate $\left(\mathrm{PO}_{3}{ }^{2-}\right)$ group of adenosine triphosphate (ATP) to the corresponding amino acid residue and protein phosphatases removes the phosphate group. Phosphate is highly negatively charged and hydrophilic group and phosphorylation can alter the charge and characteristic of proteins that lead to conformational changes and consequently cause effects on its functional outputs such as its activity, localization, stability, and interactions with other proteins (Figure 7). ${ }^{52}$ Since phosphorylation modifies protein functions, this mechanism plays a critical role for many processes in cells such as growth, division and regulation of signal transduction pathways. ${ }^{53-55}$

\section{Functional outputs}

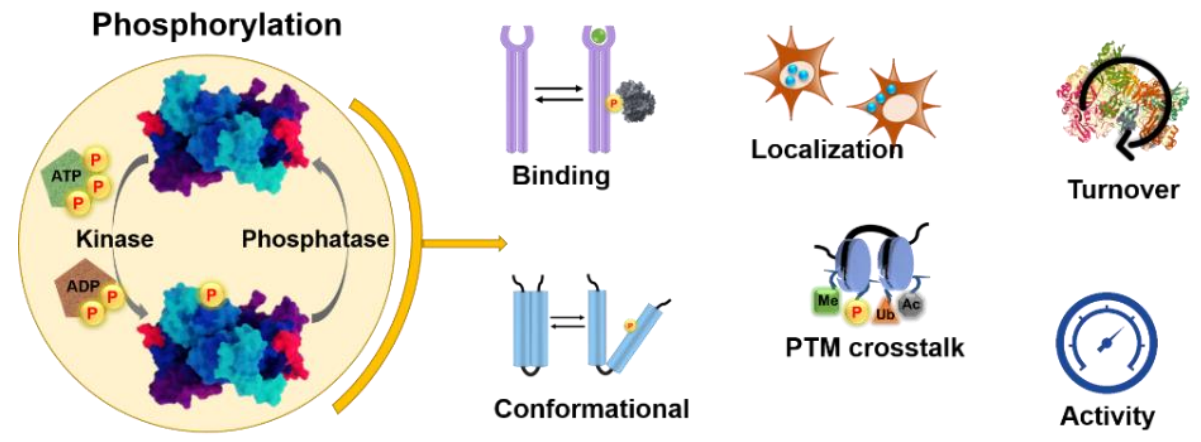

Figure 7: Protein phosphorylation regulated by switch mechanism of kinases and phosphatases and the functional outputs of this particular mechanism such as conformation, localization, binding, interaction with other proteins, PTM crosstalk (Me, Ub, and Ac stand for methylation, ubiquitination, and acetylation, respectively). Adopted from Humphrey et al. ${ }^{52}$ with permission from Elsevier. 
It is estimated that there are over 500 protein kinases, one of the largest gene family calculated about $2 \%$ of the genome, and around 140 phosphatases presented in humans. ${ }^{56,57}$ Protein kinases are generally classified in two subunits, which are dualspecificity of serine/threonine kinases that phosphorylate proteins containing serine (Ser, S) or threonine (Thr, T) residues and tyrosine kinases that phosphorylate proteins, have tyrosine (Tyr, Y) residues. Protein phosphorylation predominantly occurs on serine followed by threonine and the lowest abundance, tyrosine. The phosphorylation ratio on these common phosphorylation events is found as 1800:200:1 (pSer:pThr:pTyr) for Ser, Thr, and Tyr residues. ${ }^{58,59}$ However, phosphorylation events is not restricted only to Ser, Thr, and Tyr. Even though the major efforts have focused on these three amino acids residues, there are rare phosphorylation events that occur to the following six amino acids residues, histidine (His), lysine (Lys), arginine (Arg), cysteine (Cys), glutamic acid (Glu) and aspartic acid (Asp) ${ }^{60-62}$ The main difference amongst these amino acids is where the phosphate group is attached in their sidechains. Ser, Thr, and Tyr are known as phosphomonoesters where the phosphate group is attached to hydroxyl oxygen (O-P bond); His, Lys, Arg are called phosphoamidate here the phosphate group bind to nitrogen (N-P bond), Cys has thiophosphate bond and; Glu and Asp make acyl-phosphate bonds. Phosphorylation on these nine amino acids are shown in Figure $8{ }^{63}$
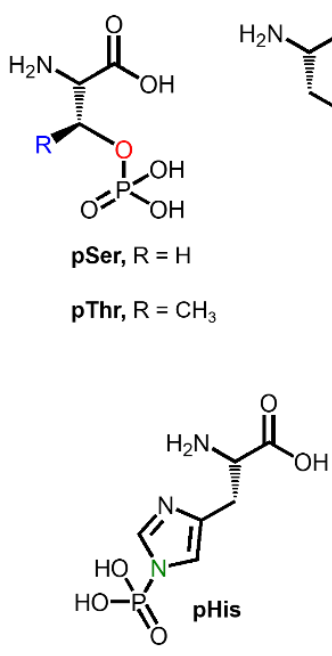
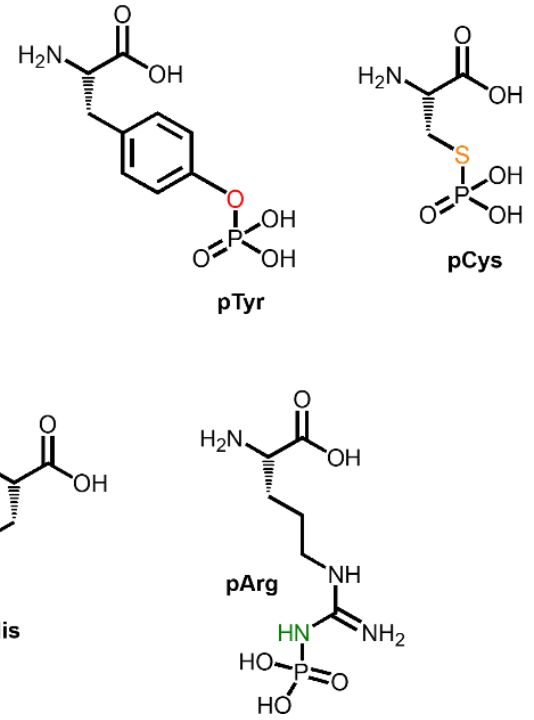
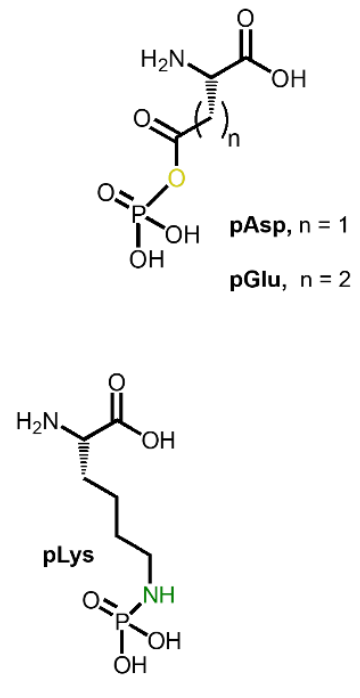

Figure 8: Phosphorylation on Ser, Thr, Tyr (O-P phosphoester bond), Cys ( $S-P$ thiophosphate bond), Asp, Glu (acyl-phosphates), His, Arg, Lys (N-P phosphoamidate bond) are shown with their chemical structure with modification. Adopted from Marmelstein et al. ${ }^{63}$ with permission from Springer. 


\section{Tyrosine Phosphorylation}

The first eukaryotic tyrosine kinase was discovered by Eckhart W., Hunter T., Sefton BM in 1979-1980 through their studies about animal tumor virus transforming protein and viral proto-oncogene tyrosine protein kinase, $\mathrm{v}-\mathrm{Src} .{ }^{64}$ Over the past 40 years, there has been an astounding progress in identification of tyrosine phosphorylation and its effects on functional outputs, which lead to the development of therapeutic drugs related with cancer. ${ }^{64}$

In historical perspective, researches on epidermal growth factor (EGF) and platelet-derived growth factor (PDGF) which are part of receptor tyrosine kinase (RTK) family play a critical role to understand the function of tyrosine phosphorylation in growth factor signaling and its relation in oncogenesis. ${ }^{52,65}$ When the SH2 domain, a phosphodependent-binding domain was discovered in 1986, the binding of this domain to a particular tyrosine phosphorylation had accelerated progress on understanding how the binding of this domain initiates downstream signaling. Furthermore, it led to a better understanding of how intracellular signaling changes when binding of this domain recruits other proteins. ${ }^{66}$ During the following years, other pTyr-binding domains (PTB), were identified such as growth factor receptor-bound protein 2 (Grb2), signal transducer and activator of transcription (STATs) in RTK signaling. ${ }^{66,67}$ In terms of signaling pathways controlled by protein tyrosine kinases (PTKs) / protein tyrosine phosphatases (PTPs), the mitogen-activated protein kinase (MAPK) ${ }^{68}$, phosphatidylinositol-3-kinase (PI3K) ${ }^{69,70}$, Janus kinase (JAK) ${ }^{71,72}$, Zeta-chain-associated protein kinase $70 \mathrm{kDa}(\mathrm{ZAP}-70)^{73-75}$ in the human kinome are some examples for tyrosine kinases.

Compared to pSer and pThr, pTyrs residue represent unique behavior, play large part for secondary interactions, and activate or mediate many biological processes from cell proliferation to aging to human diseases. Why do phosphate groups have an extreme attraction to tyrosine and why do tyrosine phosphorylation have as high potential for further cellular activities? This might be originated from the high bond energy of phosphate ester and phenolic ring in tyrosine providing additional hydrophobic or $\pi$ bond-ring interactions in a chemical perspective. Having much deeper binding pockets, $\mathrm{SH} 2$ domain tend to bind pTyr residues preferentially and specifically than pSer/pThr. ${ }^{76}$ On the other hand, even though tyrosine kinases are relatively large number ( $\sim 90$ human TK), total tyrosine phosphorylation is less than $1 \%$. Unlike $\mathrm{pSer} / \mathrm{pThr}$, tyrosine phosphorylation is essential in protein regulation rather than structural activities. In addition, PTPs have high turnover rate, which makes the half life of pTyr shorter if it is not protected. ${ }^{77,78}$ 


\section{Histidine Phosphorylation}

Oxidative phosphorylation on histidine (pHis) was firstly discovered and scientifically reported by Paul D. Boyer research group in $1962 .{ }^{79} \mathrm{pHis}$ was found significant for prokaryotic signal transduction and an intermediate for metabolic enzyme. Understanding of its function in mammalian system is one of the hot-topic in many research group. Phosphorylation of histidine is based on attachment of phosphate group to imidazole nitrogen atoms (N1 or N3) presented in the histidine structure, and forms highenergy phosphoamidate (P-N) bonds. This phosphoamidate formation can also occurs on lysine and arginine amino acid residues. ${ }^{61}$ Unlike pSer, pThr, and pTyr and their more stable phosphoester bonds (P-O), the high free energy of hydrolysis of $\mathrm{P}-\mathrm{N}$ bond makes pHis relatively unstable and its stability $\mathrm{pH}$-dependent. ${ }^{60,80,81}$ For example, the detection of histidine phosphorylation involves very or slightly acidic treatment which can cause losing the phosphate group (Figure 9) ${ }^{81}$

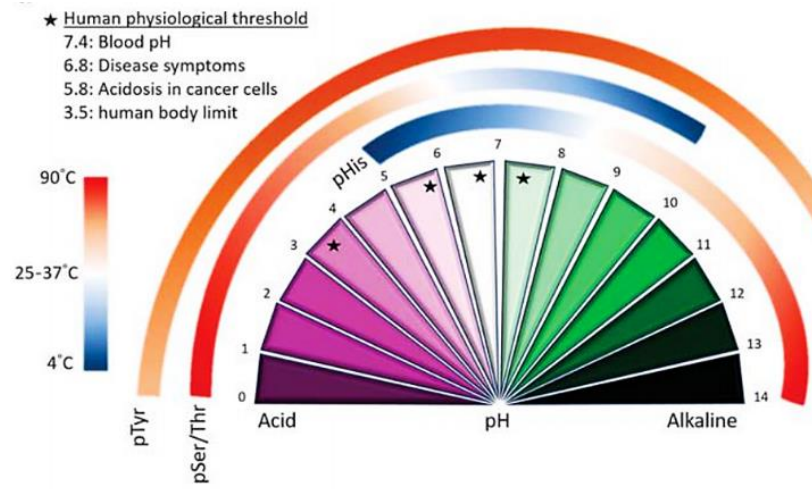

Figure 9: The stability profiles of phosphorylation on tyrosine, serine/threonine phosphoesters and histidine phosphoamidate under different $\mathrm{pH}$ and temperature. Adopted from Hunter et al. ${ }^{81}$ with permission from Nature, Laboratory Investigation.

Since the imidazole ring has two nitrogen, pHis can occur in three different forms. Two different isomeric mono-phosphohistidines: 1-pHis ( $\pi$-pHis) and 3-pHis ( $\tau$-pHis) and one diphospho structure: 1,3 -diphosphohistidine ( $\pi, \tau$-pHis) (Figure 10a). The location of the phosphate group also affects the stability of this particular modification. Between two isomeric forms, 3-pHis found more hydrolytically stable than 1pHis ${ }^{82}$ Comparison of half-lives in between $\mathrm{pH} 4$ and 6, 1-pHis hydrolysis was found ten times more rapid than 3-pHis due to its close positioning to positively charged $\alpha$ nitrogen which increase the electrophilic property of the phosphate group and make easier to dephosphorylate. ${ }^{82}$ 

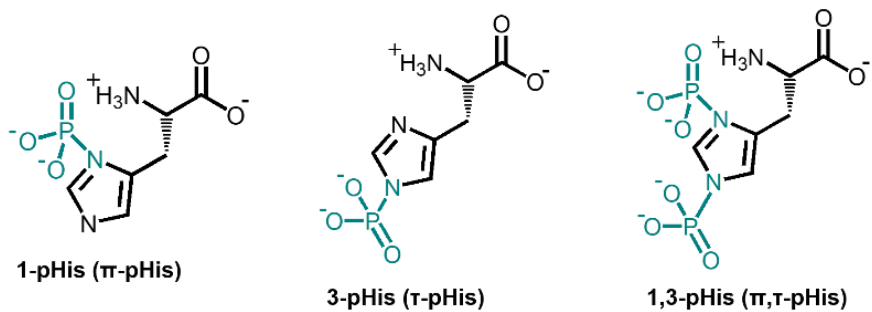

Figure 10: The structures of 1-phosphohistidine, 3-phosphohistidine, and 1,3-diphosphohistidine.

In terms of phosphohistidine proteomics, this acid-labile histidine phosphorylation was estimated as $6 \%$ of total protein phosphorylation in eukaryotic cells by H.R. Matthews who investigated regulator of the mitogen-activated protein kinase cascade in 1995. ${ }^{83}$ This value is still not showing the complete estimation of how large pHis proteome is because of common challenges for identification of histidine phosphorylation such as neutral loss of phosphate and lower ionization efficiency. In terms of the biological role of histidine phosphorylation, it was found that pHis involved in two-component regulatory system excessively including histidine kinase domain in eukaryotes. ${ }^{81,84,85}$ These two component regulatory system are based on an inner membrane-spanning histidine kinase and a cytoplasmic response regulator. In that system, typically a membrane bound histidine kinase autophosphorylates as a response to an extracellular stimuli, phosphorylation and dephosphorylation on histidine can change in changing environmental conditions such as $\mathrm{pH}$, temperature. It has also been shown that histidine phosphorylation has a main role in the sugar phosphotransferase system in bacteria. ${ }^{85}$ In mammalian cells, Nucleoside Diphosphate Kinase 1/2 (NME1/2) were reported as two highly related proteins with the activity of histidine protein kinases and Phosphohistidine Phosphatase 1 (PHPT1) as potential phosphatases activity. ${ }^{86}$ Compared to Ser, Thr, and Tyr phosphorylation and their roles in altering protein conformations, recruiting other proteins or to be preserved by locking domains (e.g. pTyr-SH2 domain), phosphorylation on histidine except involving in enzyme intermediates could in theory participate in the regulation of electrostatic protein-protein interactions. Due to chemical instability and isomerism of pHis residues, detection and analysis of pHis require special efforts such as synthesis of stable pHis analogues to mimic unstable ones, exploring and optimizing mass spectrometry (MS) targeting particularly phosphorylation on histidine, better capture materials and enrichment techniques prior to MS. Despite recent remarkable progress in the histidine phosphorylation research ${ }^{60,80,87,88}$, there are still challenges that need to overcome for studying this elusive PTM and understand its biological role. 


\section{The Role and Significance of Protein Phosphorylation in Cancer}

Reversible protein phosphorylation is responsible for the regulation of many important processes such as cell proliferation, differentiation, and apoptosis, which are vital in signal transduction pathways. The level of cellular kinase phosphorylation is firmly regulated and controlled by kinases and phosphatases. Dysregulation of these processes has been described to contribute to oncogenesis and might result in constitutive activation of different signaling pathways where other signaling proteins and secondary domains are involved. ${ }^{89-91}$ Moreover, this dysregulation might play a critical role for the survival and spread of cancer cells since kinases are involved in misrelated expression, amplification, mutations, chromosomal translocation, genetic or epigenetic altering where there might be many malfunctioning of signaling network leading to cancer. One of the most known kinase is serine/threonine-protein kinase BRAF, BRAF gene that is a class of oncogenes (Figure 11). ${ }^{92}$ When this kinase get mutated, it has potential to cause normal cells to become cancerous. BRAF is one of the common mutated kinase in human cancer and occurred approximately $~ 40-60 \%$ in melanoma, $10-15 \%$ colorectal cancer, and $1-5 \%$ of non-small cell lung cancer.

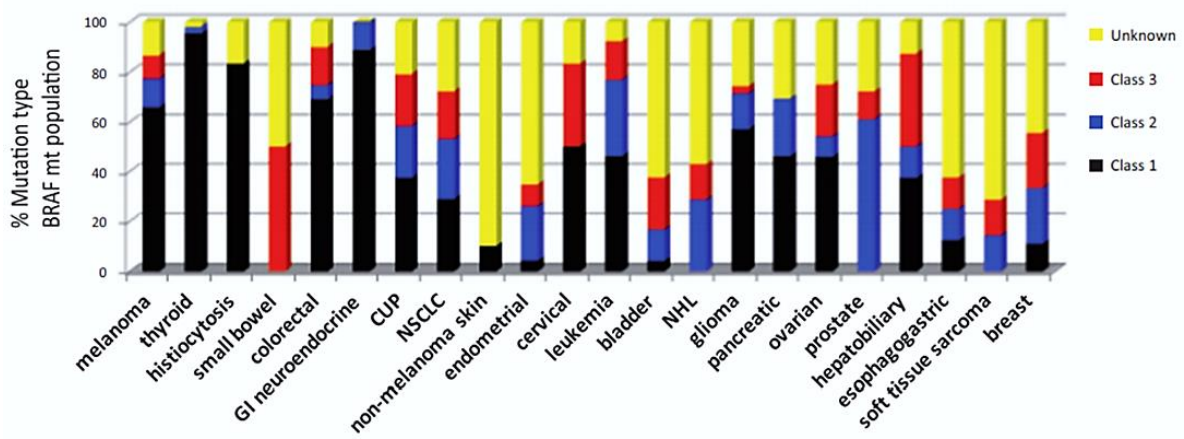

Figure 11: Three classes of BRAF mutations presented in different type of cancers. Class 1: where the strong activation of BRAF's kinase activity, Class 2: Intermediate to high kinase activity, Class 3: where the kinase activity is low or lack of mutation presented. Adopted from Watson et al. ${ }^{92}$ with permission from Nature, Oncogene.

In terms of tyrosine phosphorylation, receptor tyrosine kinases (RTK) were identified as receptor-specific ligands such as when EGFR binds to extracellular regions of RTKs, it activates the receptor and conformational changes allow auto-phosphorylation followed by recruiting other pTyr specific domains. ${ }^{93,94}$ The altering cellular behavior and activation of signaling proteins via tyrosine kinases has no doubt on dominating oncoprotein status that result the oncogenic activation of tyrosine kinase, which play a transforming role in cancer states. 
The discovery of proto-oncogene Src (sarcoma) and its interaction with focal adhesion kinase, FAK (a substrate of $\mathrm{v}$-Src oncogene) is a well-known example for tyrosine phosphorylation of FAK by Src affects cell shape, adhesion and mobility. ${ }^{95-97}$ In terms of binding interaction, when FAK phosphorylated at particular tyrosine positioned at Y397, it recruits by Src-SH2 domain and Src activity increases the maximum phosphorylation level of FAK via autophosphorylation of other tyrosine residues positioned at Y576, Y577, Y861, and Y925 (Figure 12).${ }^{97}$ In the FAK-Src complex, the phosphorylated FAK at Y861 is associated with another binding domain SH3 (Src Homology 3) where proline-rich regions are available. Phosphorylated FAK at Y925 leads Grb2 adaptor protein to be recruited and this regulate extracellular signal-regulated kinase-2 (ERK2) / mitogen-activated protein kinase (MAPK) cascade. This dynamic is a key to modulate not only cell motility but also proliferation of signals in the cell.

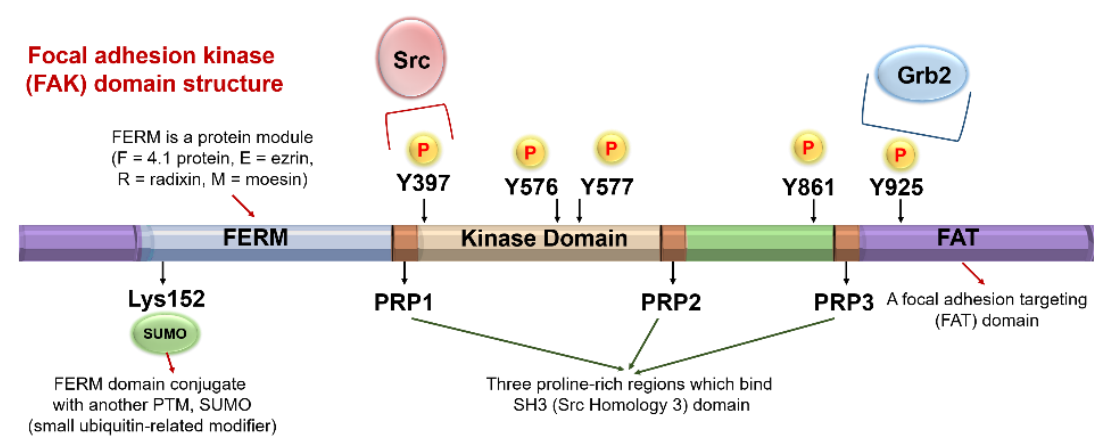

Figure 12: Focal adhesion kinase domain (FAK) structure, phosphorylation sites and binding activities. Adopted from Schlaepfer et al. ${ }^{97}$ with permission from Springer.

Many downstream signaling pathways including MAPK, PI3K and JAK/STAT and overexpression of various growth factor receptors such as EGFR and IGFRs (insulinlike growth factor receptor) are also found associated and vital in various facets of cancer progression. ${ }^{98,99}$

The genetic mutations in kinases and their disease relation are not limited with cancer but also linked with neurodegenerative disease ${ }^{100}$, diabetes ${ }^{101}$, cardiovascular disease ${ }^{102}$, and inflammatory diseases ${ }^{103}$. For this reason, there has been considerable effort and intensive research in identification of drivers for kinases and potential candidates for disease biomarkers. Exploring the role of kinases has not only helped in drug discovery, but also kinase-targeted cancer therapy ${ }^{104-107}$ from monoclonal antibody to development of small-molecule agents known as kinase inhibitors and to immunotherapies and even personalized medicine applications. 
In terms of kinase-targeted therapies, there are many kinase inhibitors used for the treatment of various human cancers due to improved clinical efficiency. Some of U.S. Food and Drug Administration (FAD) approved Ser/Thr kinase targeting and Tyr kinase targeting inhibitors shown below. ${ }^{104}$
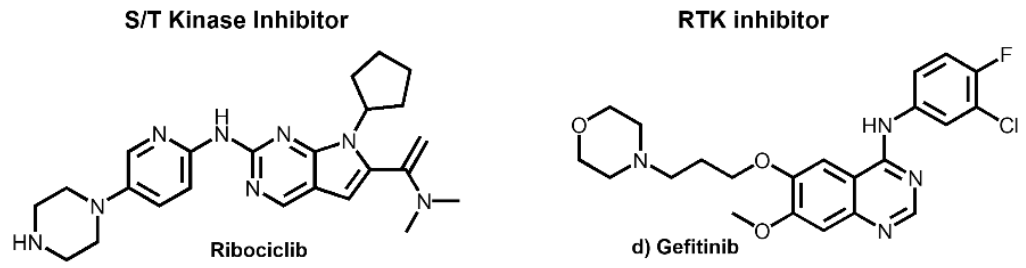

Small molecule kinase inhibitors were classified regarding to their mechanism of action. ${ }^{104}$ For instance, while Type I kinase inhibitors compete for substrate, binds to ATP binding pocket of active conformation, and alter conformational site; Type II kinase inhibitors interact with the catalytic site of nonphosphorylated inactive conformation. There are also other types, allosteric inhibitors (Type III), substrate directed inhibitors (Type IV) and covalent inhibitor (Type V). ${ }^{104}$

Except these small molecule kinase inhibitors, kinase-targeted antibodies have also shown promising efficiency in many cancer types such Trastuzumab is an example, it is a recombinant humanized IgG1 monoclonal antibody against human epidermal growth factor receptor 2, HER-2 receptor and used in breast cancer. ${ }^{108}$

Interestingly, some natural bioactive compounds are also studied and identified as kinase inhibitors. Mostly polyphenol based complexes with antioxidant property such as curcumin and green tea extracts exhibited EGFR inhibition. ${ }^{109}$

Despite the great number of studies on kinase-targeted cancer treatment, cancer is incredibly complex, dynamic interconnected systems with many different clonal heterogeneity in signaling pathways due to different kinases involved. One important issue is the kinase inhibitor resistance, which means that cancer stem cells show resistance to chemotherapy and radiation. For this reason, developing kinase inhibitors with high tolerance to mutations, introducing alternative binding sites, targeting other possible pathways in the chance of any kinase transformation can overcome challenging on resistance to kinase inhibitors. 


\section{Enrichment and Characterization of Protein Phosphorylation}

Given the importance of biological role of phosphorylation, identified many number of kinases, phosphatases enzymes, different isoforms of phosphorylation sites in particular amino acid residues, and advances of phosphoproteomics on disease biomarkers, target drugs; it is essential to develop analysis techniques for complete understanding of the role of dysregulated phosphorylation mechanism for early diagnosis and effective treatment of diseases. A comprehensive analysis of phosphoproteomics consists of enrichment of phosphorylated proteins and peptides, identification of phosphorylated samples, localization of exact amino acid residue that has phosphate prosthetic group as well as quantification of phosphorylation. There are current approaches for the analysis of phosphorylation based on mass spectrometry (MS) such as 'top-down MS' perform at the level of intact proteins and 'bottom-up MS' that perform at the level of peptides. ${ }^{110-113}$ The analysis of phosphoproteomics either protein or peptide level face with some challenges mostly related with physicochemical properties of the phosphorylated residues and possible limitations in characterization techniques. Phosphate group itself causes low ionization efficiency hence phosphogroups are negatively charged and they tend to lose protons to carry negative charges. Phosphoproteomes are in general less abundant species due to dynamic kinase and phosphatase activity compared to their nonphosphorylated forms present in much greater quantities that makes phosphorylated residues suppress in the background via presence of large amount of counterparts, consequently affecting MS analysis. For these reasons, in order to increase abundance of phosphoproteins or phosphopeptides, sample preparation based on enrichment and/or fractionation prior to MS is essential and crucial to be implemented to reduce the sample complexity and to allow more specific phospho-enrichment. ${ }^{56,114,115}$

MS-based phosphoprotemics workflow commonly start extracting of proteins from biological samples (Figure 13). Phosphoprotein enrichment is performed immediately after extraction of protein sample or peptides can be obtained by proteolytic digest of proteins and then phosphopeptide in mixture can be carried out for enrichment. In terms of enrichment strategies, affinity-based chromatography, phospho-specific antibodies and protein domains, or polymer-based capture materials can be used prior to MS. ${ }^{115}$ Moreover; these methods can be combined for better coverage of phosphoproteomes. Enriched fractions are then analyzed using MS based techniques such as matrix assisted laser desorption/ionization (MALDI)-MS or liquid chromatography (LC)-MS. The vast majority of phosphoproteomics is based on bottom-up MS technique for identification and quantification of phosphopeptides. 

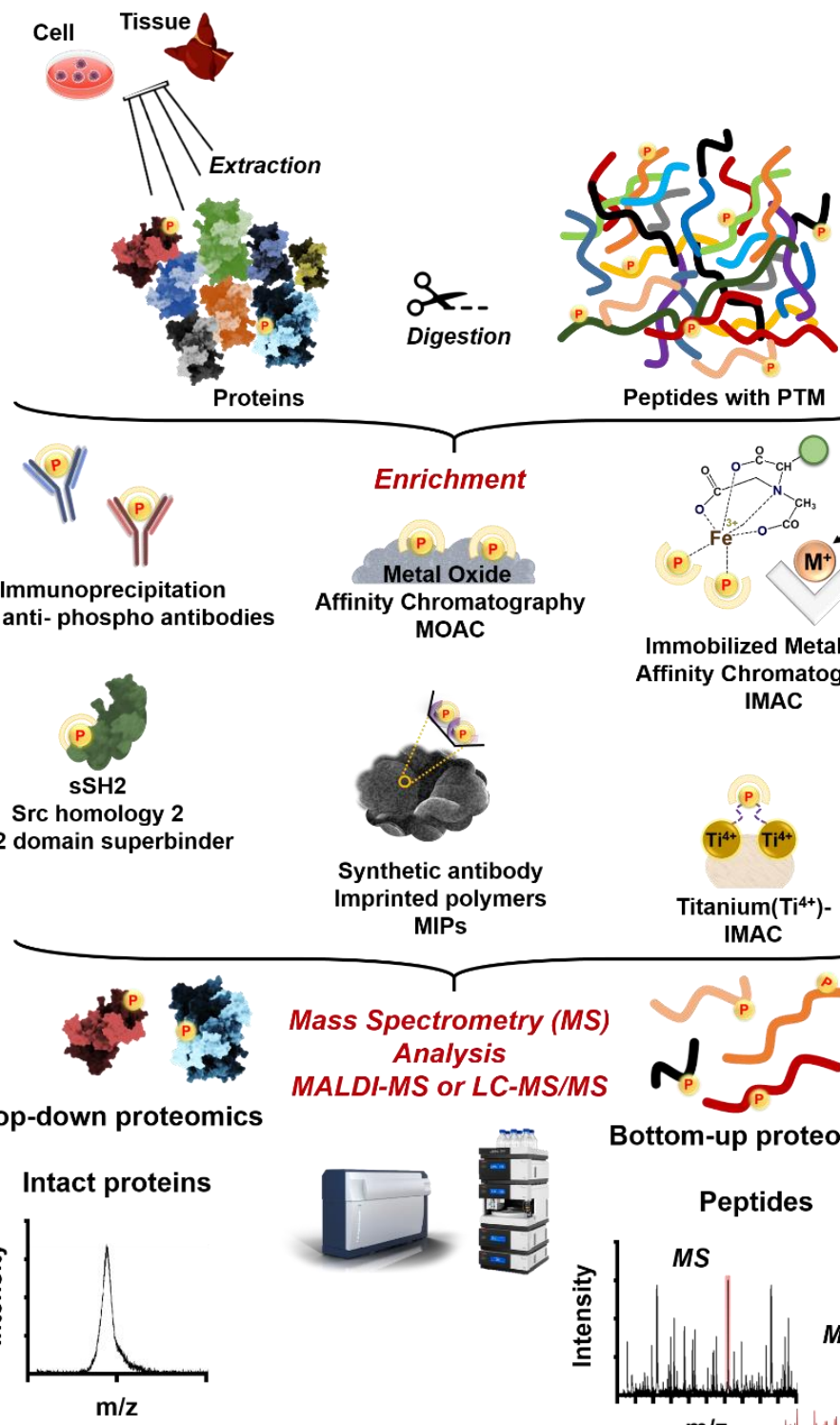

Peptides with PTM

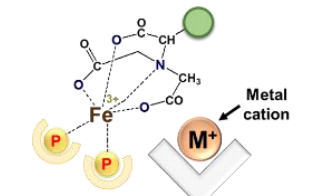

Immobilized Metal lons

Affinity Chromatography IMAC

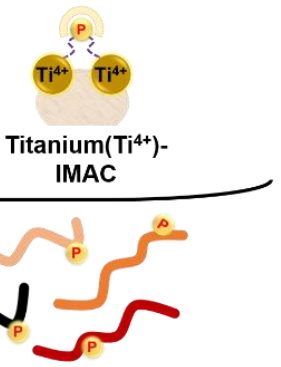

Bottom-up proteomics

\section{Peptides}

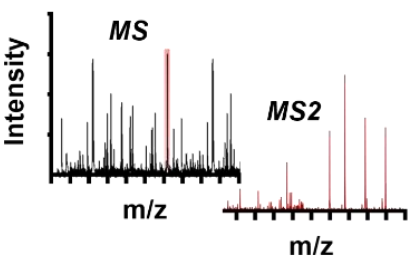

Figure 13: Phosphoproteome enrichment strategies and analysis workflow: Proteins are extracted from biological samples. Enzymatic digest is performed prior to enrichment. Phosphoproteins or phosphosphopeptides enrichment can be done using different techniques (immunoprecitation, IMAC, MOAC, SH2-superbinder, Ti-IMAC, biomimetic polymers) shown in enrichment part. Enriched phosphorylated samples are then analyzed using mass spectrometry based instrumentations (MALDI-MS or LC-MS/MS) for identification and quantification. 


\section{Enrichment Strategies}

\section{Immobilized Metal Ions Affinity Chromatography (IMAC)}

IMAC consists of a supporting matrix composed of a chelating group associated with metal cation. The ability of metal ions to interact with phosphate group is via electrostatic attraction. High valence metal cations ${ }^{116-120}$ such as $\mathrm{Fe}^{3+}, \mathrm{Ga}^{3+}$, or $\mathrm{Ti}^{4+}$ and $\mathrm{Zr}^{4+}$ are noncovalently immobilized on the supporting matrix using chelating agents such as iminodiacetic acid (IDA) and nitrilotriacetic acid (NTA) shown in Figure 14a. ${ }^{121}$ This method had been used for the efficient extraction of Ser, Thr, and Tyr based phosphorylated proteins and peptides. Since the interactions between phosphorylated targets and IMAC binders based on Lewis acid-base reactions, $\mathrm{pH}$ of binding plays a significant role. For this reason, this method suffers from nonspecific binding of especially acidic peptides containing aspartic acid or glutamic acid where carboxylate moiety interacts with metal ion materials. To avoid the nonspecific binding, acidic loading and washing steps are usually carried out to protonate acidic amino acid residues; however, highly acidic condition might leach out the chelating agent from supporting matrix.
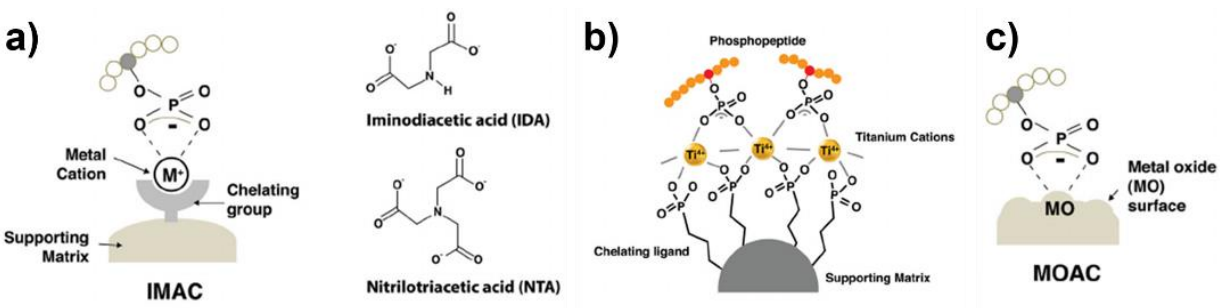

Figure 14: Affinity chromatography techniques schematically presented: a) IMAC (metal ion immobilized affinity chromatography, b) Ti(IV)-IMAC (Titanium-IMAC) and c) MOAC (metal oxide affinity chromatography). Adopted from Mingliang et al. ${ }^{121}$ with permission from Wiley.

Not only metal (III) cations, but also $\mathrm{Ti}^{4+}$ have been extensively used for IMAC (Figure 14b). $\mathrm{Ti}^{4+}-$ IMAC showed more strong coordination with both phosphates and carboxylic acids. Additionally, using new chelating like phosphate $\left(-\mathrm{PO}_{3}{ }^{2-}\right)$ or arsenate $\left(\mathrm{AsO}_{3}{ }^{2-}\right)$ in the design improved loading capacity, robustness, selectivity, and resistant to leaching compared to other IMAC materials. Apart from enrichment of phosphoesters, $\mathrm{Fe}^{3+}$-IMAC and $\mathrm{Cu}^{2+}$-IMAC were also used for the enrichment of acid-labile phosphorylation on histidine peptides. ${ }^{122}$ Moreover, there is an increase attention on using lanthanide elements such as $\mathrm{Gd}^{3+}, \mathrm{La}^{3+}$, and $\mathrm{Er}^{3+}$ due to their tight ionic bonds formation towards phosphates. ${ }^{123}$ 


\section{Metal Oxide Affinity Chromatography (MOAC)}

MOAC matrix composed of metal oxides (MO) or hydroxides where it can form stable bonds toward oxygen anions (Figure 14c). ${ }^{121}$ MO-based affinity materials show higher tolerance for acidic $\mathrm{pH}$ than IMAC. The MO interacts with phosphate groups forming bidentate interaction. Titanium oxide $\left(\mathrm{TiO}_{2}\right)$ is the frequently used $\mathrm{MO}$ for the enrichment of phosphopeptides. ${ }^{124,125}$ The manufacturing of $\mathrm{TiO}_{2}$ particles play a critical role in terms of binding capacity since the morphology in particles effect the surface-to-volume ratio. While $\mathrm{TiO}_{2}$ was used for phosphopeptide enrichment, aluminum hydroxide $\left(\mathrm{Al}(\mathrm{OH})_{3}\right)$ was used for enrichment of phosphoproteins. In addition, zirconium dioxide $\left(\mathrm{ZrO}_{2}\right)$, gallium oxide $\left(\mathrm{Ga}_{2} \mathrm{O}_{3}\right)$, ferric oxide $\left(\mathrm{Fe}_{3} \mathrm{O}_{4}\right)$, niobium oxide $\left(\mathrm{Nb}_{2} \mathrm{O}_{3}\right)$ and stannic oxide $\left(\mathrm{SnO}_{2}\right)$ also carried out for phosphoproteforms enrichment. ${ }^{126-128}$ Comparison of $\mathrm{TiO}_{2}$ and $\mathrm{ZrO}_{2}$ materials, it was found that $\mathrm{TiO}_{2}$ is more selective for mono-phosphorylated residues while $\mathrm{ZrO}_{2}$ for multi-phosphorylated forms. ${ }^{129}$

Even though both IMAC and MOAC present similar properties such as binding mechanism towards phosphate, the combination of these enrichment techniques bring advantages to enhance the phosphoproteome coverage. This approach is based on using first $\mathrm{Fe}^{3+}$-IMAC and then $\mathrm{TiO}_{2}$ that is called sequential elution from IMAC (SIMAC). ${ }^{130,131}$ Following, peptide mixture is treated with $\mathrm{Fe}^{3+}$-IMAC, then the unbound and the fraction washed with acidic solution are separately collected for enrichment of mono-phosphorylated peptides using $\mathrm{TiO}_{2}$. The bound peptides are eluted using basic conditions from $\mathrm{Fe}^{3+}$-IMAC beads for the enrichment of multiple-phosphorylated peptides.

\section{Immunoprecipitation and Superbinders}

When foreign molecule called as antigen enter body, antibody is produced by the immune system. Antibodies bind specifically those antigens and it can cleared from system. Immunoprecipitation (IP) is to isolate a specific antigen from complex mixture using its specific antibody and is used for enrichment of phosphoproteins or phosphopeptides using antibodies against phosphorylated residues. ${ }^{132,133}$ There are commercially available and well-studied antibodies such as pTyr-100 and 4G10 to immunoprecipitate pTyr proteins. ${ }^{121}$ In contrast, phospho-selective antibodies against pSer and pThr have cross-reactivity, consequently not efficient binding for all $\mathrm{pS} / \mathrm{pT}$ site. Due to limitation of antibody-based affinity such as requiring large amount of sample, high cost for sufficient amount and requiring special storage conditions, alternative, costeffective binding reagents was used to perform enrichment. ${ }^{134}$ 
SH2 domain derivative pTyr binding materials, called SH2-superbinders, showed great potential to be used in the enrichment of pTyr peptides. ${ }^{121}$ Since SH2 domain as a sequence-specific phosphotyrosine binding module represents high affinity to a broad range of pTyr peptides, one-step SH2 superbinder eliminating commonly used phosphopeptide enrichment techniques showed significant improvement in pTyr peptides purification, consequently better identification of concentrated and purified residues from complex mixture. SH2 superbinder modified monolithic capillary column showed unique identification of more than 700 pTyr sites using only $100 \mu \mathrm{g}$ of $\mathrm{HeLa}$ cell digest (Figure 15). ${ }^{135}$ This work represented a significant improvement on phosphotyrosine enrichment.

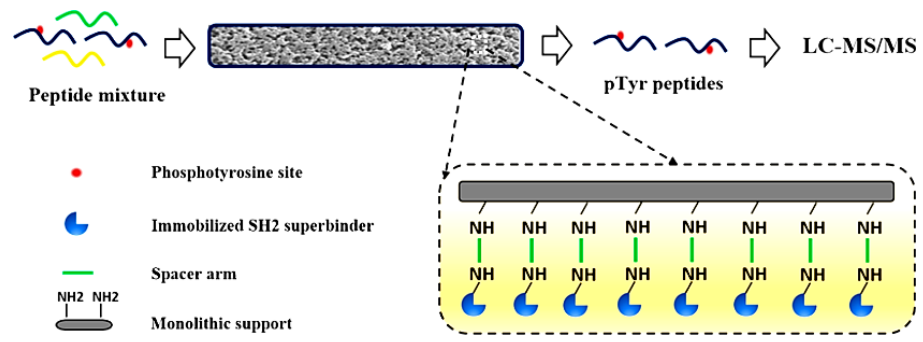

Figure 15: The concept of SH2 superbinder modified monolithic capillary column for selective enrichment and analysis of pTyr from small amount of cell digest. Adopted from Yao et al. ${ }^{135}$ with permission from ACS.

Except affinity chromatography, immunoprecipitation, and $\mathrm{SH} 2$-superbinder, there is also chemical derivatization, which is based on modification of phosphorylated residues prior to enrichment. ${ }^{15} \beta$-elimination is one of example and this process consists of treatment peptide mixture with alkaline conditions where pSer and pThr undergo facile dephosphorylation via $\beta$-elimination in a strongly alkaline environment resulting dehyroalanine and $\beta$-methyldehydroalanine residues, respectively. This reaction combined with subsequent Michael addition for enrichment of pSer and pThr peptides. This approach can be applied to enrich acid-labile phosphorylation on histidine in the presence of base-labile phosphoesters. However, this method is not applicable for tyrosine phosphorylation since pTyr does not undergo $\beta$-elimination. Another one is phosphoamidate chemistry (PAC). This derivatization can be applied to all phosphorylated residues. The concept consists of multi-step chemical modification and is based on reconstituting phosphate group by acid hydrolysis of the phosphoamidate bonds such synthesis of N-phosphorylation of histidine residues using potassium phosphoramidate, or lysine-containing peptides. Due to requiring multiple reaction steps for this derivatization, this technique might cause losing sample in each step. 
Not only these more specific enrichment strategies, but there is also fractionation techniques such as strong cation exchange (SCX), strong anion exchange (SAX), and hydrophilic interaction chromatography (HILIC) which are less selective compared to previously mentioned enrichment techniques. Both SCX and SAX are based on an ion exchange chromatography principle. ${ }^{136}$ In SCX, phosphopeptides eluted earlier from anionic stationary phase, on the contrary, phosphopeptides are strongly retained in SAX due to cationic stationary phase. These both techniques are often used for prefractionation of phosphopeptides prior to enrichment using IMAC or $\mathrm{TiO}_{2}{ }^{59-137}$ HILIC where neutral hydrophilic stationary phased used separate peptides according to their hydrophilicity / hydrophobicity in the acetonitrile-water system. ${ }^{136}$

\section{Imprinted Polymers}

Imprinted polymers, MIPs, earlier mentioned in the previous chapter are known biomimetic polymers had been used to develop phospho- specific affinity materials for enrichment of phosphorylated residues from complex mixture. ${ }^{138-141}$ The very first example on this concept was reported by B. Sellergren in $2008 .{ }^{138}$ Imprinted polymer synthesized via stable complexes formed between the diaryl-urea host monomer and quaternary ammonium phosphate salt showed promising results for targeting pTyr peptides and bias on target peptide in the presence of non-phosphorylated tyrosine and serine counterparts. Using this strategy and optimization later on facilitate the enrichment of pTyr peptides spiked in complex digest. Not only pTyr peptides, but also using designed and synthesized particular template in the synthesis of pSer-specific MIPs showed a complementary selectivity towards phosphoserine-targeting peptides. In another study, four methods including pTyr-MIP, $\mathrm{TiO}_{2}$ and their combinations were used to evaluate and compare in terms of binding specificity towards different phosphorylation sites. ${ }^{142}$ Recently, the study on selective enrichment of histidine-phosphorylated peptides using MIPs (Paper II) represented the design of three different pHis-targeting imprinted polymers and validated selective enrichment of short pHis peptides using high-crosslinked polymers, and larger pHis-peptides using hierarchical MIP (synthesized using silica particles as scaffold in the synthesis of polymers). ${ }^{143}$ In another study, zinc acrylate as functional monomer was used together with phenylphosphonic acid (PPA) as template for the synthesis of imprinted material and thIS study showed promising bias for pTyr peptides in aqueous buffer compared to $\mathrm{TiO}_{2}$ performance in buffer. ${ }^{144}$ 


\section{Characterization - MS-based phosphoproteomics}

\section{Mass Spectrometry (MS)}

Mass spectrometry (MS) is a powerful and versatile analytical tool that is used to identify and quantify of samples and elucidate the structure and chemical properties of molecules. Briefly, the discovery of mass spectrometry was by J. J. Thomson about his initial characterization of electron and he was awarded by Nobel Price 1906. ${ }^{110,145}$ Then continues studies on electric and magnetic field, the beams of positive ions, fast ions and reionization under the vacuum, F. W. Aston firstly described and measured $\mathrm{m} / \mathrm{z}$ values and he was awarded by Nobel Price in 1922. Describing and studying on isotopes for chemically identical atoms, atomic weight measurements, relative abundance, ion-acceleration, double focusing electrostatic, magnetic deflection, high accuracy of isotopic masses; the molecular mass spectrometry method and improvements on this field became very popular during 1925-1950. After 1950s, the qualitative molecular mass spectrometry and careful studies on MS fragmentation of various type of samples accelerated the mass spectrometry growing as a field all around the world. ${ }^{110,145}$ After the revolutionary of analytical devices such as gas chromatography (GC) and liquid chromatography (LC) and their combination with MS techniques increased the advantages of identification enormous number of compounds. In 1966, chemical ionization by F. H. Field and M. S. B. Munson, in 1968 electrospray ionization (ESI) by M. Dole, and in 1985, matrix-assisted laser desorption-adsorption (MALDI) by F. Hillenkamp, M. Karas and co-workers were developed. In 1989, the development of ion trap technique by W. Paul brings him the Nobel Price in Physics. Then, A. Makarov presents the Orbitrap MS in 1999. In $21^{\text {th }}$ century, J. B. Fenn and K. Tanaka were awarded of the Nobel Price in chemistry for the development of soft ionization technique for the MS analyses of biological macromolecules. ${ }^{110,145}$

Mass spectrometer measures the mass-to-charge $(\mathrm{m} / \mathrm{z})$ ratio of ionized atoms or group of atoms. MS is widely used in proteomic analysis for both top-down MS to sequence intact proteins and bottom-up MS to facilitate identification of short peptides obtained by proteolytic digest of proteins (Figure 13). MS-based phosphoproteomics is fundamental to uncover signaling networks in a variety of biological samples, profiling PTMs, localization, and simple quantification of large-scale phosphoproteoforms. MS is composed of three parts: ion source, mass analyzer, and detector (Figure 16). Ionization determines different types of analytes to be measured by combination of mass analyzer and detector that determine the quality, reliability and efficiency of process. 


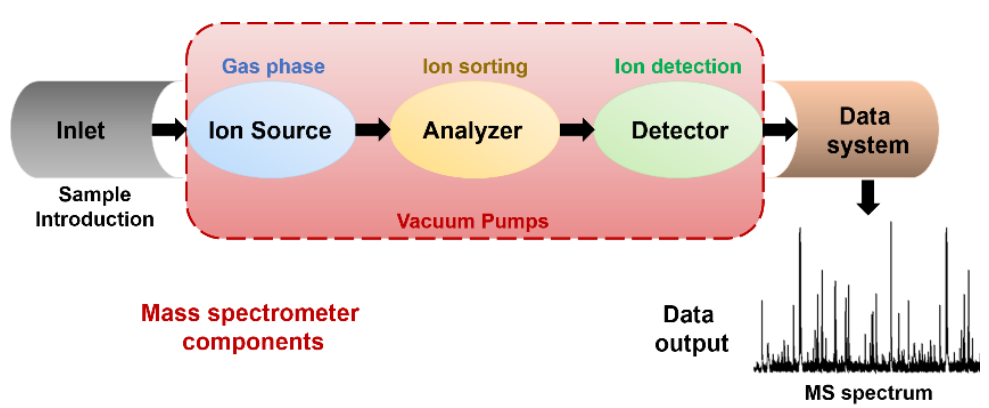

Figure 16: Mass spectrometer components: Inlet, ion source, mass analyzer, detector, data system (data output and data handling).

\section{Ionization}

Ionization of samples in MS is simply where the mechanism of ionization occurs via ionization source. After sample is placed in an instrument and inserted into ionization source via direct insertion, direct infusion or injection followed any number of desorption process such as laser or heat, vaporization and ionization can be facilitated. In ionization process, different methods can be used such as protonation (formation of positive ions), deprotonation (removal of one proton), cationization (charged complex adding positively charged ions e.g. ammonium ion), transfer of a charged molecule to gas phase, electron ejection (ejected electron to get position ion) or electron capture (absorption of an electron). Protonation $\left(\mathrm{M}+\mathrm{H}^{+} \rightarrow \mathrm{MH}^{+}\right)$is a method where positive charge of +1 produces. Since positive charges via protonation make more stable form together with basic residues, peptides are often ionized via protonation.

Two most commonly used ionization methods are MALDI and ESI shown in Figure 17. ${ }^{146-148}$ These two techniques are widely used in proteomic studies that help to generate gas-phase analyte ions to provide efficient and non-destructive ionization of samples.
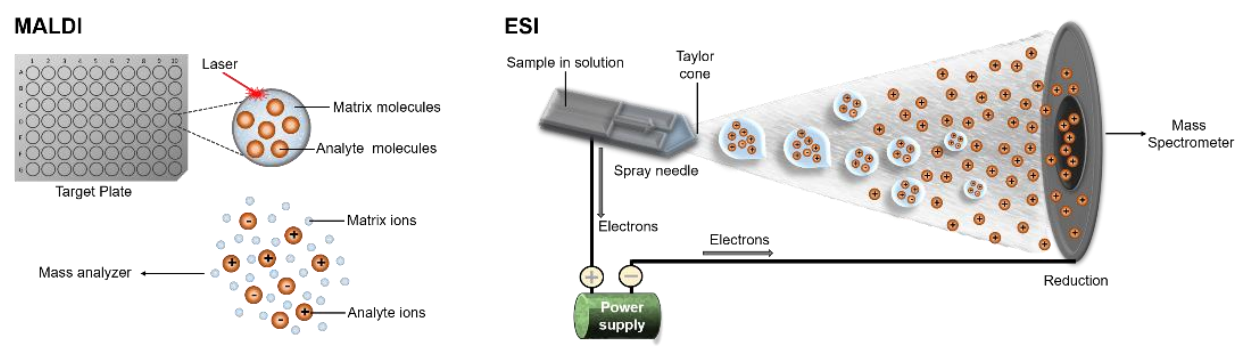

Figure 17: Mass spectrometry ionization techniques are shown in schematic. Matrix-assisted laser desorption/ionization (MALDI) on the left and Electrospray ionization (ESI) on the right. 
In MALDI, the sample is mixed with excessive amount of ultra-violet absorbing matrix with low molecular weight such as 2,5-hydroxy benzoic acid (DHB) or 3,5-dimethoxy-4-hydroxycinnamic acid (sinapinic acid) and then deposited on a metal plate. When laser irradiates, the matrix then absorbs the radiation and converts to heat energy; therefore, it facilitates both desorption and ionization process of the sample. ${ }^{147}$

In ESI, the gas phase ions are formed via passing sample in solution through a capillary maintained at high voltage. Due to strong electrical field, Taylor cone on the tip of spray needle generates formation of aerosol droplets. When the droplets evaporate, they turn into smaller droplets; this process continues concentrating charged droplets to ions. ESI is compatible to be interfaced with liquid chromatography (LC) and it can be coupled with MS. ${ }^{148}$

Both MALDI and ESI are soft ionization methods, which is very useful for proteomics studies since the analyte do not lead to fragmentation.

\section{Mass Analyzer}

The ionized sample is then introduced to mass analyzer that separate and resolve gas phase molecule ions into their characteristics mass components according to their $\mathrm{m} / \mathrm{z}$ ratio using some physical property such as electric or magnetic field. The mass analyzer properties include accuracy (measure of how close the value obtained to the true value that varies from analyzer type), mass range, resolution (measure of how good a MS separates ions of different masses), and scan speed. There are different mass analyzer which can be classified regarding to how ions being introduced such as continuous or pulsed modes. Commonly used mass analyzers in proteomics are Time-ofFlight (TOF), Ion trap, Fourier transform ion cyclotron resonance (FT-ICR), Quadrupole, and Orbitraps. ${ }^{149}$

\section{Time-of-Flight (TOF)}

TOF mass analyzer is a pulsed and non-scanning MS that consists of an accelerator, a field-free region, a reflectron ion mirror, and detector inside the flight tube. TOF MS is based on separation and detection of ions according to their $\mathrm{m} / \mathrm{z}$ ratio by measuring the time for the ions to travel through a field-free region. In principle, accelerated ions via high acceleration voltage generates unique kinetic energy and velocity for particular ions. Each ion flies inside the flight and then reach to ion detector. While the lower $\mathrm{m} / \mathrm{z}$ value ions flies faster, the larger masses flies slower. TOF can be converted to $\mathrm{m} / \mathrm{z}$ to generate mass spectrum. ${ }^{150}$ 
TOF analyzer have two modes that are linear and reflectron (Figure 18). Linear mode is where ions reach to detector at different times. Even though this mode results in low resolution of a one broad peak representing as non-resolved isotope peak (Figure 18a), it has advantages to be used for identification of high molecular samples or the analyte are not stable enough to survive the applied energetic field in the reflectron. Reaccelerating of ions requires high $\mathrm{kV}$ electric field and this high field effects larger molecules e.g. intact proteins that can undergo fragmentation. Reflectron mode provides high-resolution spectrum show all isotope of peaks well-separated and mass accuracy. Introducing reflectron ion mirrors equipped with repulse back electric field into drift zone where the ions are refocused by their masses which helps to ions to reach the detector simultaneously (Figure 18b).
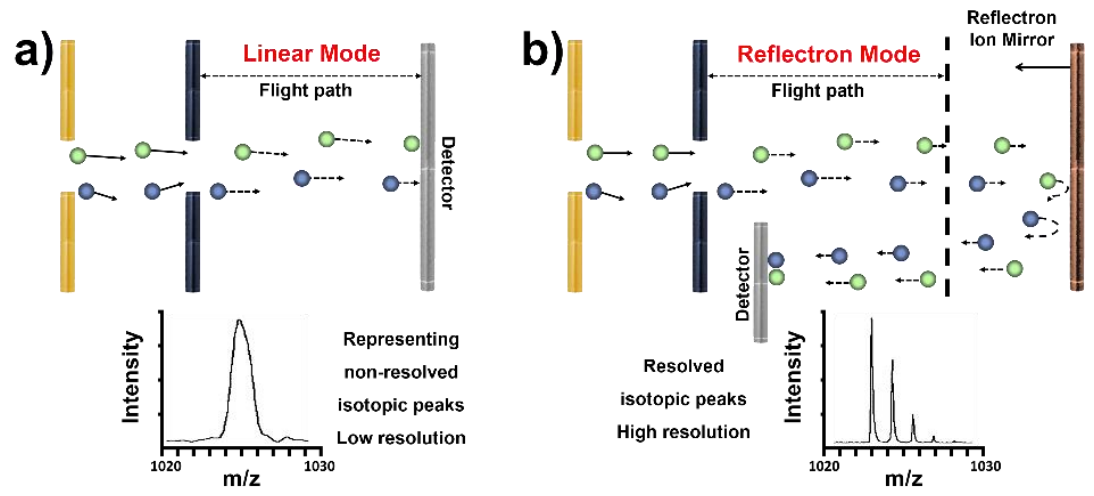

Figure 18: Schematic representation of a) linear mode and b) reflectron mode in TOF analyzer.

TOF analyzer is usually coupled with MALDI that measure masses of both proteins and peptides with simplicity, mass accuracy, high resolution and sensitivity.

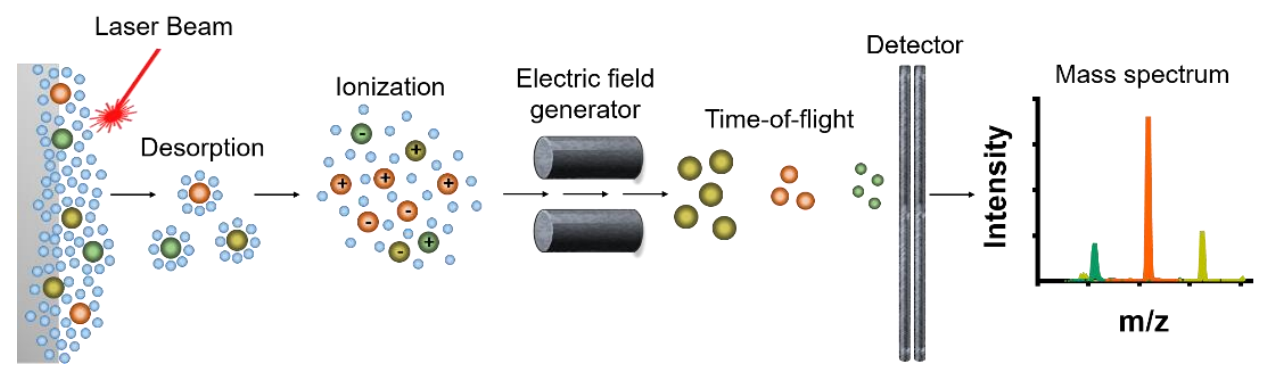

Figure 19: Schematic representation of MALDI-TOF/MS instrument.

MALDI-TOF/MS instrument was used in Paper II, III, and V for quantification of peptide mixture and profiling of fractions in enrichment. 


\section{Quadrupole, Ion Trap and Orbitrap}

The quadrupole mass analyzer (Figure 20a) ${ }^{149}$ based on continuous scanning mass filter, which consists of four cylindrical metal rods parallel to each other in the vacuum chamber. The opposing rod pair is connected together electrically. By generating a direct currents and alternate currents, ions with target $\mathrm{m} / \mathrm{z}$ value pass through the quadruple and then get to the detector. Varying the voltage allows different masses to pass in turn and then mass spectrum can be generated. Quadrupole mass filter can scan through all masses via mass scanning mode or can sit at one fixed mass via single mass transmission mode. LC-MS-ESI instrument with quadruple mass analyzer was used in Paper II and V.

Ion trap mass analyzer (Figure 20b) ${ }^{149}$ is based on ion trapping and pulsed MS. Ion trap analyzer consists of same ion sources like in quadrupole except quadrupole device is replaced with ion trap analyzer. Therefore, ion trap represents three-dimensional device where it can store ions. This can allow getting more information for the ions with lower $\mathrm{m} / \mathrm{z}$ values. For these reason, mass spectra obtained via ion trap are richer in fragmentation ions compared to quadrupole MS.

Orbitrap mass analyzer (Figure 20c) $)^{149}$ is a type of ion trap analyzer where the ions are trapped around central spindle electrode that holds the trap together and aligns it via dielectric-end spacers. The idea of orbitrap is based on composing of all previously described mass analyzers such as trapping and the shaped of electrodes from ion trap, pulsed injection and electrostatic fields from TOF. Using external pulsed ion source, ions are trapped and then ions pushed to orbitrap. Increasing the voltage in the central electrode provides electrodynamic squeezing and ions are trapped. By detection the oscillation of ions, frequencies introduced to get $\mathrm{m} / \mathrm{z}$ values of ions via their oscillation frequencies. The Orbitrap Fusion Lumos Tribrid ${ }^{151}$ instrument combines with LC-MS/MS was used for Paper II and III.
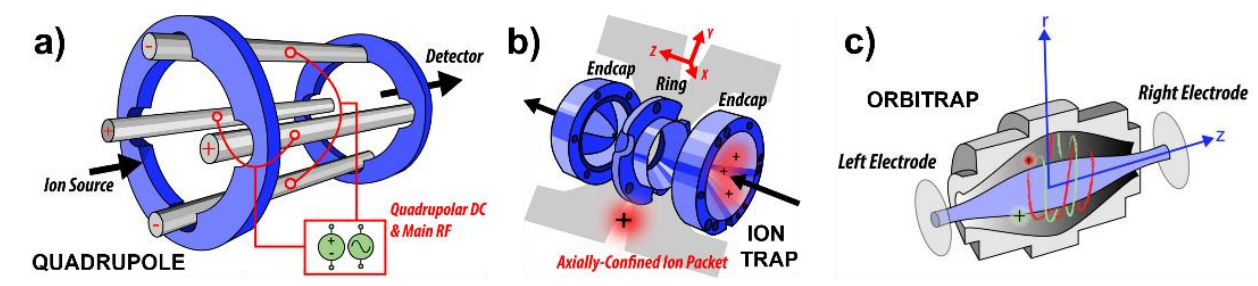

Figure 20: Schematic representation of Quadrupole (a), 3D-Ion Trap (b), and Orbitrap (c). Adopted from Kelleher et al. ${ }^{149}$ with permission from Wiley. 


\section{Detector}

Faraday cup, photomultiplier conversion dynode, array detector, charge (or inductive) detector, and electron multiplier are commonly used ion detectors in MS. ${ }^{152}$ The choice of ion detectors depends on design of instrument and particular experiment to perform. All of them is based on same principle that is generating a signal from incident ions by generating secondary electrons, which are further amplified, or by inducing current ions. In Faraday cup, the stroked incident ion emits electrons and induces a current, which is amplified and recorded. In electron multiplier, two different types can be introduced. One is same principle as Faraday cup, the second occurs due to continuous amplification through repeated collisions. The most common detector used in MS is photomultiplier. The incident ions generate electron emission and these electrons strike a phosphorous screen which turn releasing of photons. Using photons extend the lifetime of the detector that makes this type of detector preferred.

\section{Tandem Mass Spectrometry}

Once samples are ionized to generate a mixture of ions, where parent (precursor) ions obtained via $\mathrm{m} / \mathrm{z}$ values of specific molecules, they are named as MS1. If the sample is unknown then knowing MS1 is not enough to get information about its structure. Tandem MS (or MS/MS, MS ${ }^{\mathrm{n}}$ ) is a technique to break down selected precursor ions into their fragments where product ions are produced. ${ }^{153,154}$ The fragmentation of ions reveal not only chemical structure but also provides information on peptide sequence or PTMs on particular amino acid residues if any. In principle, the selected precursor ions are isolated in narrow $\mathrm{m} / \mathrm{z}$ window and then fragmentation can be done by one or several fragmentation methods to generate different product ions. The fragmentation method is based on dissociation processes that are collision-induced dissociation (CID) $)^{155}$, higher-energy C-trap dissociation (HCD) ${ }^{156}$, electron capture dissociation $(\mathrm{ECD})^{157}$ and electron transfer dissociation (ETD) ${ }^{158}$. CID uses an inert gas there energy generated to break down and it is usually coupled with triple quadrupole where first one selects a given ion and accelerates to second one that has collision cell where fragmentation occurs, and finally the third one scans the fragments. HCD principle is similar with CID but it is more specific for Orbitrap instruments. The generated higher-energy collisions produce more efficient fragmentation for lower mass fragments. ECD and ETD compare to CID and HCD are softer fragmentation techniques. ETD and ECD induces fragmentation by transferring electrons to higher state cationic molecules. ETD is mostly coupled to ion trap-Orbitrap while ECD is primarily used in FTICR. 
In the fragmentation of peptide, peptide bonds break down N-terminus fragments as $\mathrm{a}, \mathrm{b}$, or c ions and C-terminus fragments as $\mathrm{x}, \mathrm{y}$, or $\mathrm{z}$ ions (Figure 21).

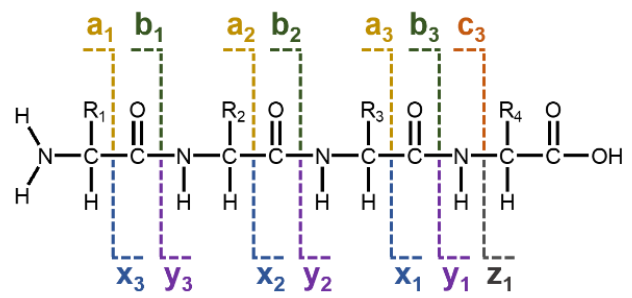

Figure 21: Ions generated due to MS/MS fragmentation: $N$-terminus as $a, b$, or c ions and $C$-terminus as $x, y$, and $z$ ions.

While CID-induced fragmentation of peptides or proteins results in N-terminal b- and C-terminal $y$-ions, ETD fragmentation occurs on the $\alpha$-C of amide bond that generates $\mathrm{c}$ - and z- ions. To get better peptide sequence information, these two dissociation processes combined and called as ETciD / EThcD which help to improve sequence coverage and confident PTM site localization.

In example Figure 22, peptide sequence identification of two model peptides that were used in Paper III represented. GADDSYpYTAR (a) and GADDpYYTAR (b) are single phosphorylated tyrosine forms of GADDSYYTAR peptide that is related with the kinase domain of zeta-associated 70-kDa protein, ZAP-70. The precursor ion mass for both peptide is $1197.43 \mathrm{Da}$. Calculating amino acid masses, not only peptide sequence but also the localization of phosphate group on tyrosine residues either $6^{\text {th }}$ (GADDpYYTAR, Figure 22b) or $7^{\text {th }}$ (GADDYpYTAR, Figure 22a) were identified since phosphate adds $80 \mathrm{Da}$ due to neutral loss of phosphate group, $\mathrm{HPO}_{3}$ in mass.
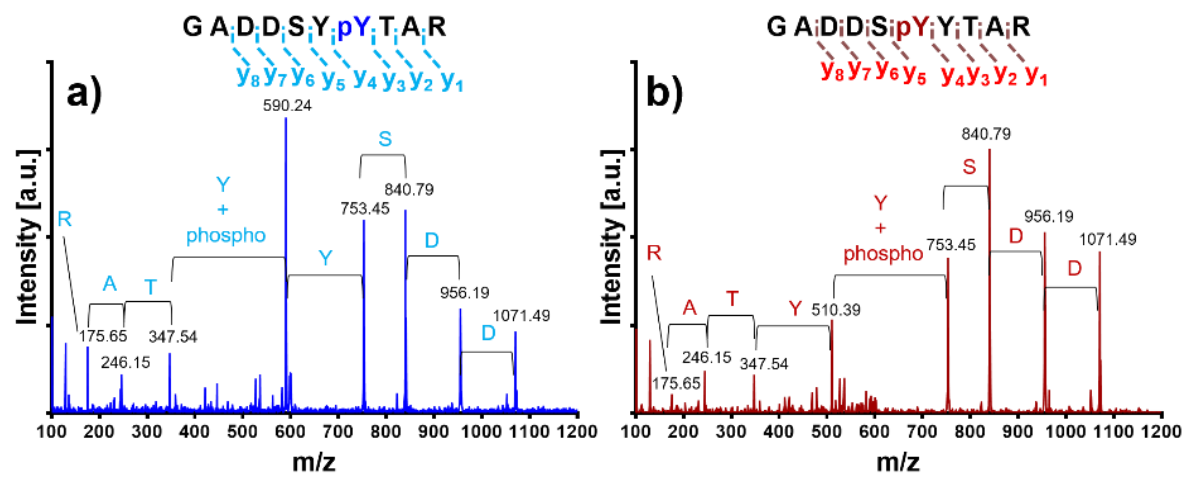

Figure 22: The fragmentation profiles of GADDSYpYTAR (a) and GADDSpYYTAR (b) peptides. 


\section{MIPS FOR PHOSPHOPEPTIDE ENRICHMENT}

\section{State of Art}

In this thesis, the objectives are to design and synthesize template molecules regarding to phospho- targets, to synthesize imprinted polymers via noncovalent approach in the presence of synthesized template molecules together with functional urea monomers using combination of epitope and stoichiometric imprinting approaches, and then to enrich target phosphorylated peptides from complex peptide mixture using these synthetic receptors.

\section{Epitope Imprinting}

In terms of imprinting of biomolecules, there are some intrinsic limitations in conventional polymerization approach to MIP synthesis due to molecular size, complexity, conformational flexibility, and solubility of larger molecules such as peptides and proteins. The traditional noncovalent approach in synthesis of imprinted polymers usually requires excess amount of crosslinking agent resulting high-crosslinked and robust materials. Not only amount of crosslinking agent, but also aprotic solvent is often chosen as porogen to provide better self-assembly between templates and functional monomers. Considering larger biomolecule imprinting under these conditions will lead generating limited binding sites, having additional disadvantages such as steric accessibility of porous materials, and excessive weak interactions due to distinct physicochemical properties of larger molecule. Moreover, when larger biomolecules are used as template, for instance proteins, they might denature, change conformations and even aggregate which make them unsuitable for imprinting.

To overcome these limitation, 'epitope imprinting' idea was developed. ${ }^{159}$ This technique relies on using a small fragment of target peptide or protein as template in polymerization resulting imprinted polymer where able to retain the whole peptide or protein. Using this alternative approach also conquer possible problems such as poor mass transfer, denaturation or heterogeneity in the binding pocket affinity. 


\section{Stoichiometric Imprinting}

The chosen functional monomers to complement around a template molecule lie in self-assembly principle where the complexation between monomers and template determines the binding groups in their positions in receptor binding sites. When targeting phosphorylated residues via MIPs, the design of imprinting is simply based on an anion recognition. Using commercially available monomers such as methacrylate or methacrylamide for the synthesis of anion targeting imprinted polymers, only weak interactions can be formed in the polymer network. ${ }^{160}$ For this reason, in this type of imprinting, usually excess amount of functional monomers being introduced to increase the degree of complexation around template. However, this condition is not compatible with many biomolecules since they might require polar or aqueous environment for imprinting. ${ }^{161}$

To improve this, design and synthesis of new functional monomers that provide strong and stoichiometric interactions with template molecule should be taken into account. Since stoichiometric complexation between template and functional monomer is strong enough, it results generating high percentage of complexation in the prepolymerization mixture, consequently, high yield of selective binding sites in polymer network and reduced degree of nonspecific binding. One of the example of stoichiometric imprinting was demonstrated by Wulff G. in 1997 with the use of amidinebased monomer in the presence of template, phosphonic acid mono ester. ${ }^{162}$

\section{Anion Binding with Urea and Thiourea Receptors}

Due to the importance of not only biological role of anions but also industrial processes and pollutants, the research on anion receptors has expanded greatly in the last few decades. ${ }^{163}$ Much effort has been devoted to synthesize anion receptor motifs such as amides, guanidium, steroids, and pyrroles. ${ }^{164}$ Especially, urea and thiourea derivatives ${ }^{165}$ played and continuous to play a leading role in the field of anion coordination chemistry. Oxyanions such as phosphate, sulfate, or carboxylate are larger, generally have a wide range of geometries and high solvation energy, and display more $\mathrm{pH}$ sensitive interactions compared to their cations, therefore they require high degree of design and complementary to make selective synthetic receptors. One of the first example of using urea derivatives for molecular recognition was reported by Hamilton in 1993. This work introduced hydrogen-bond donating urea hosts capable of recognizing carboxylate guests. ${ }^{166} \mathrm{Up}$ to now, a variety of oxyanion receptors containing urea and thiourea subunit have been developed and studied to understand the significant role of this unique group in the anion recognition. ${ }^{165,167}$ 
Urea and thiourea moiety are potent hydrogen bond (H-bond) donors and hydrogen atoms on the nitrogen can interact with oxygen atoms in oxyanions creating an eightmembered cyclic structure (Figure 23a). ${ }^{165}$ Not only oxyanions, but also halides make complexation forming a six-membered ring via interaction between bifurcated $\mathrm{H}$ bonds and anion in halide complexes (Figure 23b). ${ }^{165}$

a)

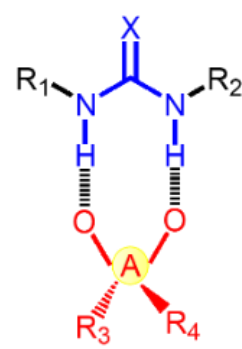

b)

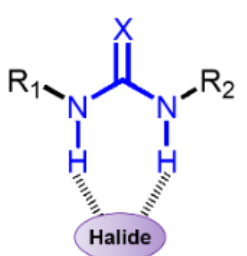

c)<smiles>[X]C(=[X]c1cccc([R2])c1)Nc1cccc([R])c1</smiles>

$\mathrm{X}=0, \mathrm{~S}$

Figure 23: Hydrogen bond orientation of urea or thiourea receptors for the binding of oxyanion $(a)$, or halide complexes $(b)$, and the main structure of diphenyl- urea or thiourea derivatives. Urea: $X=O$ and Thiourea: $X=S$.

H-bond stabilization in the formation of anion-urea or anion-thiourea complexes hence relies on where $\mathrm{H}$-bond acceptors of the anion interacting with $\mathrm{H}$-bond donor of the receptor. For this reason, the strength of $\mathrm{H}$-bonds, consequently the stability of complexation, relies on the acidity of the receptor and the basicity of guest. The unsubstituted thiourea $(\mathrm{pKa}=21.1 \pm 0.1)$ has lower $\mathrm{pKa}$ value than urea $(\mathrm{pKa}=26.9 \pm 0.1)$ which makes it more acidic. In terms of acidic strength, diaryl urea derivatives (Figure $23 \mathrm{c})$ such as $\mathrm{N}, \mathrm{N}^{\prime}$-diphenyl- thiourea $(\mathrm{pKa}=13.4 \pm 0.1)$ are more acidic than dialkyl urea $(\mathrm{pKa}=18.7 \pm 0.1){ }^{165}$ Since the acidity of urea or thiourea derivatives is the key to the strong anion binding, the receptors with aromatic substituents compared to those carrying alkyl groups have attracted more attention; notably this type of derivatives has been extensively used in catalysts defining the field of organocatalysts. ${ }^{168}$

The choice of aryl substituents in 1,3-diaryl-ureas or thioureas also play a critical role in the design of receptors. Derivatives with a nitrophenyl group is one of the most common examples. The presence of $-\mathrm{NO}_{2}$ not only increased the acidity of the $\mathrm{NH}$ bond in the receptor, but also led to chromogenic behavior useful for colorimetric sensors. ${ }^{169}$ In the case of acidic hosts in contact with basic anion guests host deprotonation is possible. For instance, excess addition of fluoride anion ( $\mathrm{F}-$-) in aprotic media to anthracene-based derivatives can deprotonate the receptor. ${ }^{170}$ Especially, the thiourea receptors compared to their corresponding urea ones have tendency to be deprotonated because of their lower pKa values in the presence of highly basic anions. 
Hence, design of a urea and thiourea based receptors involves pKa adjustments via careful tuning of the aryl substituents to match the pKa of the guest. For these reasons, comparing many structural motifs, the strongest interactions with oxyanion guests were achieved using electron-withdrawing trifluoromethyl, $\mathrm{CF}_{3}$ moieties appended to the aryl groups. ${ }^{168,171}$ The most well-known ex-ample of using this $\mathrm{CF}_{3}$ moiety was studied by Schreiner in $2002^{171}$ with the synthesis of diarylthioureas together with 3,5bis(trifluoromethyl) groups in both sides of aromatic structure.

The -CF3 moiety is simply an electron-withdrawing group (EWG) and it makes the acid unit more acidic by delocalizing the charges leading to stronger interactions in the presence of an anion guest. ${ }^{172,173}$ The influence of the number of $\mathrm{CF}_{3}$ groups on the urea pKa is shown in Figure 24a-d. ${ }^{173}$ From dialkyl to diaryl and from two-unit $\mathrm{CF}_{3}$ to four-unit $-\mathrm{CF}_{3}$ decreases the $\mathrm{pKa}$ value of a particular receptor with nearly five units. Not only the presence of $-\mathrm{CF}_{3}$ but also its position in the aromatic groups influences the pKa values (Figure $24 \mathrm{e}, \mathrm{f}$ ).
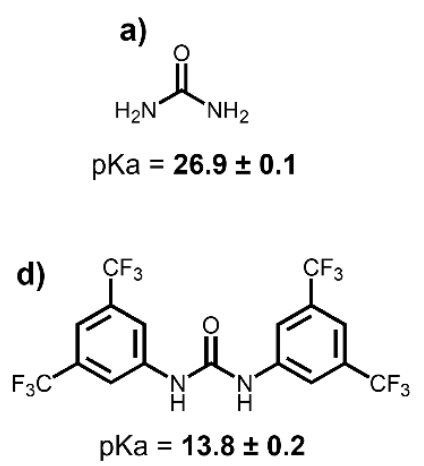

b)<smiles>O=C(Nc1ccccc1)Nc1ccccc1</smiles>

e)<smiles></smiles>

c)<smiles>O=C(Nc1ccccc1)Nc1cc(C(F)(F)F)cc(C(F)(F)F)c1</smiles>

f)<smiles>FC(F)(F)c1cc(NC(=S)Nc2ccccc2)cc(C(F)(F)F)c1</smiles>

pKa $=10.7 \pm 0.1$

Figure 24: Comparison of the effect of $-\mathrm{CF}_{3}$ moiety on $\mathrm{pKa}$ values in urea and thiourea derivatives: a) urea, b) 1,3-diphenyl-urea, c) 1-(3,5-Bis-trifluoromethyl-phenyl)-3-phenyl-urea, d) 1,3-Bis-(3,5bis-trifluoromethyl-phenyl)-urea, e) 1-(3,5-Bis-trifluoromethyl-phenyl)-3-phenyl-thiourea, f) 1 (3,5-Bis-trifluoromethyl-phenyl)-3-phenyl-thiourea. Adopted from Schreiner et al. ${ }^{173}$ with permission from ACS.

Studies have shown that the -CF3 moiety not only acidifies the receptor but also strengthens potential $\pi-\pi$ interactions through the highly polarized aryl groups. This makes -CF3 a preferred substituent when engineering diaryl-urea based receptors. 


\section{Urea-based Functional Monomers}

Emphasizing the importance of 1,3-disubstituted urea structures, their receptor properties for targeting oxyanions, and the effect of functional units introduced in receptor, the model was employed for the synthesis of functional monomers for targeting protein phosphorylation. Regarding to the objectives in this thesis, the synthesized functional monomer was used for the designing and synthesizing of amino acid side chainand sequence- specific synthetic receptors for target phosphorylated residues.

In this thesis, in terms of $\mathrm{HG}$ association and complementing functional groups of template molecules, especially targeting phosphorylated residues, 1,3-disubstituted urea monomers have been chosen as neutral hosts for making complexation in the presence of oxyanion guest. ${ }^{161,174}$ The structural and compositional parameters for tuning HG association strength for the synthesis of urea-based functional monomers shown in Figure 25. ${ }^{174}$

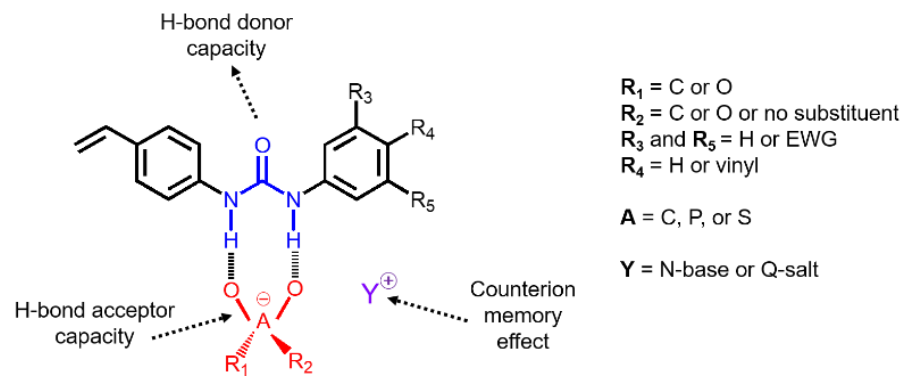

Figure 25: Structural and compositional parameters for tuning host-guest association. EWG: electron withdrawing group, $C=$ carbon, $P=$ phosphate, $S=$ sulfur, $H=$ hydrogen, $O=$ oxygen, $N=$ nitrogen, $Q=$ quaternary.

These neutral hosts provide a 2-fold H-bond donor towards affinity for guest where the affinity increases with the acidity of urea receptors and the basicity of oxyanion. To enhance the acidity of receptor, consequently $\mathrm{H}$-bond donating capacity, $-\mathrm{CF}_{3}$ moiety introduced in ortho- and para- positions of one of the aromatic ring $\left(\mathrm{R}_{3}\right.$ and $\left.\mathrm{R}_{5}\right)$. The strength of interaction between functional monomer and oxyanion also depends on solvent polarity. The advantage of this type of functional monomers is to be able to solubilize in aprotic organic solvent where it can maximize the complexation between template and functional monomer in noncovalent imprinting strategy. Following this approach to synthesize of urea receptors, it was reasoned that this idea would fulfill the tight and selective binding sites required for the synthesis of imprinted polymers for targeting phosphorylated residues. 
The core of this thesis relies on the use of unsymmetrical substituted aromatic urea derivative, 1-(4-vinylphenyl)-3-(3,5-bis(trifluromethyl) phenyl)-urea (1) functional monomer which was synthesized by the condensation of 1,3-bis(trifluromethyl) phenyl isocyanate and 4-amino styrene (Figure 26). ${ }^{161}$

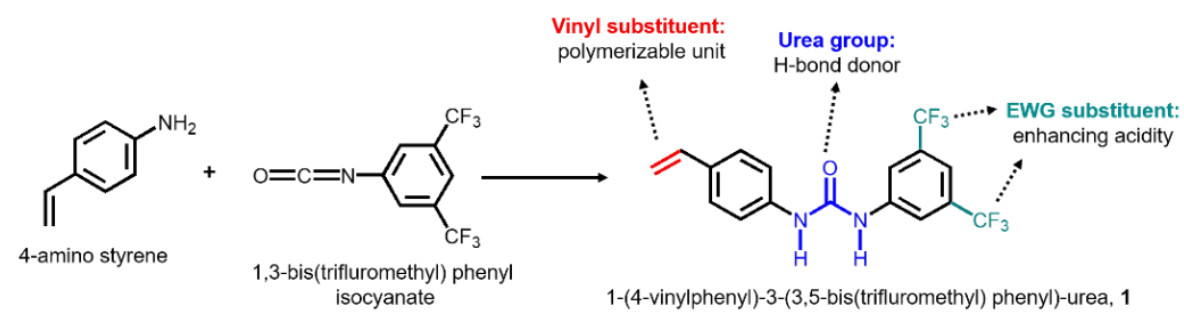

Figure 26: Synthesis of 1-(4-vinylphenyl)-3-(3,5-bis(trifluromethyl) phenyl)-urea (1) functional monomer by the condensation of 4-amino styrene and 1,3-bis(trifluromethyl) phenyl isocyanate.

Considering the oxoanion and oxourea complexation, this urea monomer, $\mathbf{1}$, form ternary HG complex with phosphate dianions (2:1 complex). To study the complexation between 1 and oxyanions and their stoichiometries, nuclear magnetic resonance (NMR) spectroscopy was used. NMR is technique to study the structure of compounds and interactions between various molecules. This spectroscopic technique is based on nuclei of atoms have magnetic properties that can be utilized to yield chemical information. Using this technique helps to determine molecular conformation in solution and physical properties at the molecular level. In this thesis, NMR was used for conformation of the structure and purity check of synthesized compounds and extensively the interaction between templates and functional monomers.

\section{Oxoanion-oxourea Complexation}

The interaction of functional urea monomer, $\mathbf{1}$ with mono tetrabutylammonium (TBA) salt of phenyl phosphonic acid (PPA) was investigated by titrating the receptor monomer solution, which was prepared in $\mathrm{d}_{6}$-DMSO (dimethyl sulfoxide), together with a standard solution of the anion guest up to a ten-fold molar excess. The spectroscopic characterization was done using ${ }^{1} \mathrm{H}$ NMR (Figure 27). ${ }^{174}$ The titration 1 with PPA $\cdot$ TBA resulted strong downfield shift in urea protons $\mathrm{Ha}$ and $\mathrm{Hb}$ which reflect the establishment of an H-bond interaction with guest molecule. The obtained data was then converted for complexation induced shift vs. free concentration of guest and fitted to a binding model to derive the complex stability constant, $\mathrm{K}_{\mathrm{eq}}$ that was calculated as $7005 \mathrm{M}^{-1}$ by fitting the model for 1:1 interaction (Paper I). 

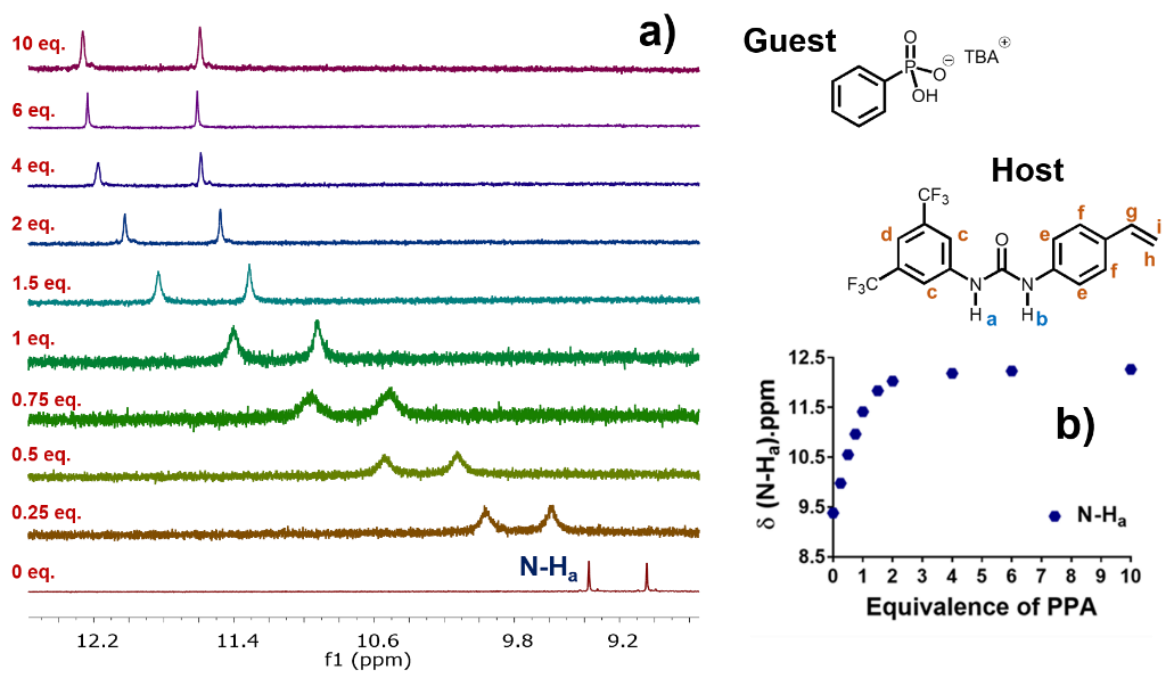

Figure 27: ${ }^{1} H-N M R$ chemical shift changes on aromatic and urea protons of host monomer 1 in DMSO-d6 with Bu4 N + $\mathrm{PhPO}_{3} \mathrm{H}-(\mathrm{PPA} \cdot \mathrm{TBA}$, guest) illustrating spectral shifts of aromatic protons (a), urea protons and the chemical shift profile of $\mathrm{N}$-Ha proton versus number of equivalents of added PPA.TBA $(b)$.

Previous studies found that urea monomers might interact with each other and they can form dimerization. This causes blocking the recognition groups that are supposed to bind guest molecule. For this reason, it is critical to understand monomer-monomer interactions too.

In Paper I, not only to understand the interaction of host $\mathbf{1}$ to another 1, but also to investigate 2:1 (2 equivalent of host: 1 equivalent of guest) binding model, twodimensional NMR with nuclear Overhauser effect spectroscopy (NOESY) was used. This technique is practical for proton resonances are located on the same or adjacent carbon nuclei. NOESY NMR of $\mathbf{1}$ confirms that there is no interaction of urea $\mathrm{N}-\mathrm{H}$ protons of monomer with another one which proves no dimerization.

To understand the complexation between $\mathbf{1}$ and bis pentamethyl piperidine (PMP) salt of PPA guest, the titration was carried out. The addition of half equivalent of PPA-2PMP to 1 caused changes in Ha protons. The visual observation had an indirect support how the addition of bis-PMP salt of PPA to 2 equivalent of 1 makes the solution of $\mathbf{1}$ in aprotic polar solvent tetrahydrofuran (THF) completely transparent (Paper I, Scheme 4). Additionally, molecular dynamic (MD) simulations completed on this binding model agreed for the degree of complexation. These studies simply confirm the 1:1 and 2:1 complexes for pre-polymerization mixture prior to imprinting. 


\section{Side Chain- and Sequence- Specific Imprinted Polymers}

Synthesis of side chain- and sequence- specific imprinted polymers were carried out using N-terminal (via fluorenylmethyloxycarbonyl (Fmoc) group) and C-terminal (via methyl or ethyl groups) protected phosphorylated templates regarding to the target.

In Paper II, 3-[4-Phosphono-[1,2,3]triazol-1-yl]-2-(9H-fluoren-9-ylmethoxycarbonylamino)-propionic acid ethyl ester (Fmoc-pTza-OEt) ${ }^{143}$ was synthesized and used as template for the preparation of imprinted polymers targeting histidine phosphorylated peptides. In Paper III, regarding to the target tyrosine phosphorylation on Y492 and Y493 amino acid residues in the kinase domain (ALGADDSYYTAR) of zeta-associated 70-kDa protein (ZAP-70), Fmoc-pTyr-Tyr-Gly-OMe, Fmoc-TyrpTyr-Gly-OMe, Fmoc-pTyr-pTyr-Gly-OMe, and Fmoc-Tyr-Tyr-Gly-OMe were synthesized and used as template for the preparation of pTyr-based sequence specific receptors. In Paper V, to mimic the behavior of Src-SH2 domain for its unique binding towards EPQpYEEIPIYL peptide, Fmoc-pTyr-Glu-Glu-Ile-OEt and Fmoc-TyrGlu-Glu-Ile-OEt were used as template for the preparation of pYEEI sequence-based recognition.

The general synthesis protocol of imprinted polymers was given in Figure 28. Acetonitrile $(\mathrm{MeCN})$ which is aprotic polar solvent was chosen as porogen. Copolymerization was carried out using pentaerythritol tetraacrylate (PETA) crosslinking agent. Conventional azo-initiated thermal polymerization was used for the formation of polymer network. After synthesis, template removal was carried out for all polymers using methanol (MeOH):0.1 M hydrochloric acid ( $\mathrm{HCl})(80: 20)$ solution.

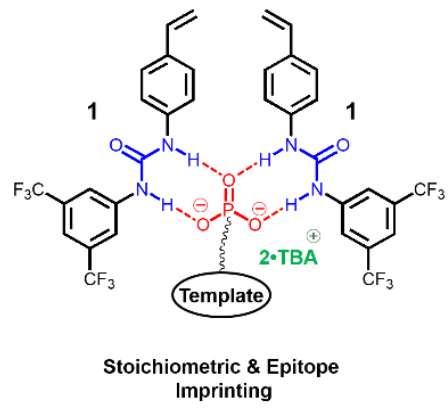

1: Functional urea monomer

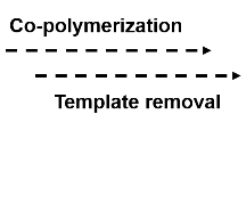

Template removal

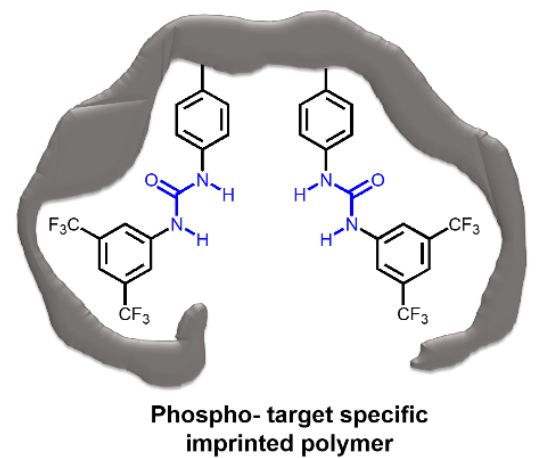

Figure 28: The general synthesis protocol of imprinted polymers using one equivalent of bis-TBA salt of phosphorylated template together with two equivalent of $\mathbf{1}$. 


\section{Static Binding}

For the evaluation of imprinted polymers, one of simple test is static rebinding (batch binding) experiment (Figure 29). This test is based on incubation of polymer particles in the presence of peptide mixture prepared at different solution. After the equilibrium, the polymer is separated from solution and the amount of unbound analyte in solution is measured using high-performance liquid chromatography (HPLC).

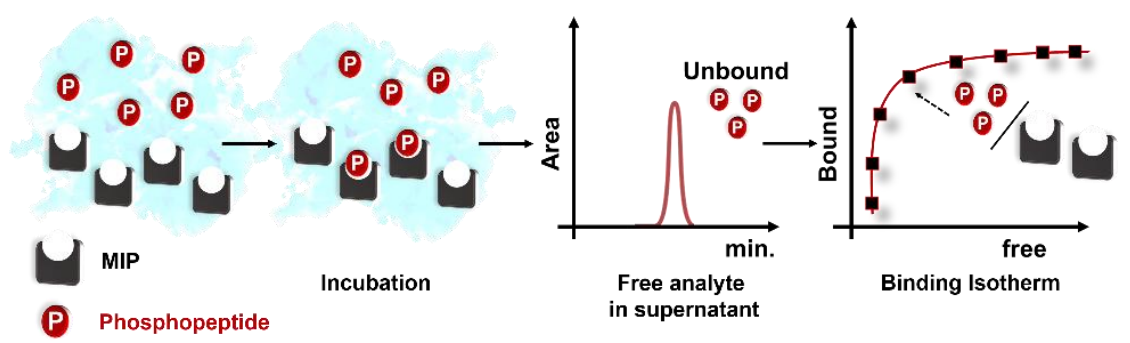

Figure 29: The rebinding experiment: Incubation polymer with analyte, determine free analyte in supernatant, calculate bound analyte, fitting model for free analyte versus bound.

HPLC is a separation technique, which allows purifying, identifying, and quantifying compounds in analytical way. Liquid chromatography is based on where the sample is forced via high pressure through a column that is packed with a stationary phase, generally composed of porous layers, by a liquid called as mobile phase. Stationary phase where the compounds are to be separated, and mobile phase which carries the compounds. The separation of individual components of a sample depends on a difference in their affinity to the stationary phase.

In the batch binding test shown in Figure 29, amount bound analyte per unit of polymer $(B$, in $\mu \mathrm{mol} / \mathrm{g})$ is calculated by followed equation: $B=\left(\mathrm{n}_{0}-\mathrm{n}_{\text {free }}\right) / \mathrm{m}_{\text {polymer }}$ where $\mathrm{n}_{0}$ is the initial concentration of analyte, $\mathrm{n}_{\text {free }}$ is the amount of unbound analyte and $\mathrm{m}_{\text {polymer }}$ is the mass of polymer used in rebinding test. The experimental data can be fitted to a theoretical model, for example Mono-Langmuir, Bis-Langmuir, Freundlich, or Langmuir Freundlich depending on the description of binding sites using a graph fitting software, for example GraphPad Prism (GraphPad Software, La Jolla, CA, USA) or OriginPro (Originlab Corporation, Wellesley Hills, MA, USA). ${ }^{175}$ Mono-langmuir model, where it is assumed that the interactions between analyte and receptor are one type of homogeneous binding, is the simplest model. The obtained adsorption isotherms calculate the maximum bound of analyte, $B_{\max }$, the dissociation, $K_{d}$, and the association constant, $K_{a}$ which is reverse of $K_{d}$ value. 


\section{Enrichment of Target Phosphopeptides}

When the imprinted polymers, which were subjected to batch binding studies, confirm the specificity towards their target phosphorylated peptides, phosphopeptide (p-peptide) enrichment can be carried out. The aim of phosphopeptide enrichment protocol is based on reducing non-target background peptides and increasing target p-peptide quantification (Figure 30).

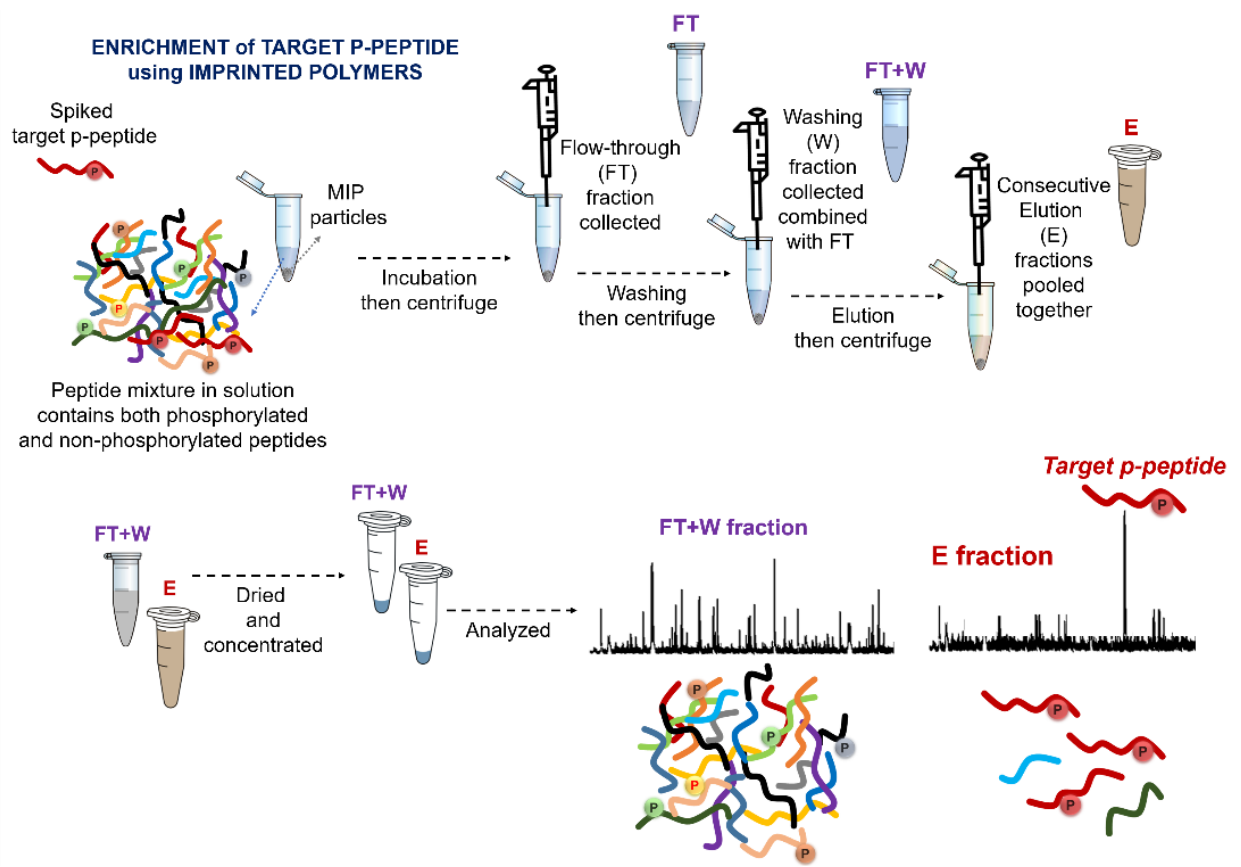

Figure 30: The protocol for enrichment of target phosphopeptide using imprinted polymer: incubation MIP particles with peptide mixture, followed by washing of particles, and then extraction of bound peptides from MIPs and then mass analysis.

First, target p-peptide is spiked into complex peptide mixture (prepared by proteolytic digest of commercially available proteins) containing both phosphorylated and nonphosphorylated residues at different spiking level that is related with the abundance of target p-peptide in total protein phosphorylation. Then incubation of MIP particles together with peptide mixture prepared in aprotic polar solvent, followed by centrifugation and flow-through (FT) fraction is collected. Next, MIP particles is washed with usually the same solution used in FT to get rid of non-specifically bound peptides from particles or container surface, followed by centrifugation and then washing (W) fraction is combined together with FT fraction. In the last step, consecutive elution 
(E) or extraction is carried to remove the bound peptides from imprinted polymers using mostly protic polar solvent and each elution fraction pooled together. Both $\mathrm{FT}+\mathrm{W}$ and $\mathrm{E}$ fractions were dried and concentrated prior to analysis. The concentrated samples were then analyzed using MALDI TOF/MS-MS or LC-MS/MS to see the peptide profile in each fraction and to quantify the bound percentage of target p-peptide.

In Paper II, to fulfill the enrichment profile of pHis-targeting imprinted polymers, $\mathrm{N}$-phosphorylation of histidine residues from horse heart myoglobin were chemically synthesized using potassium phosphoramidate chemistry. After conformation on phosphorylation via mass determination, the histidine-phosphorylated peptides were obtained by proteolytic digest (Figure 31) and the digest were mixed together with commercially available bovine serum albumin (BSA) and $\beta$-casein digest at equimolar and carried out for enrichment using pHis MIPs. ${ }^{143}$

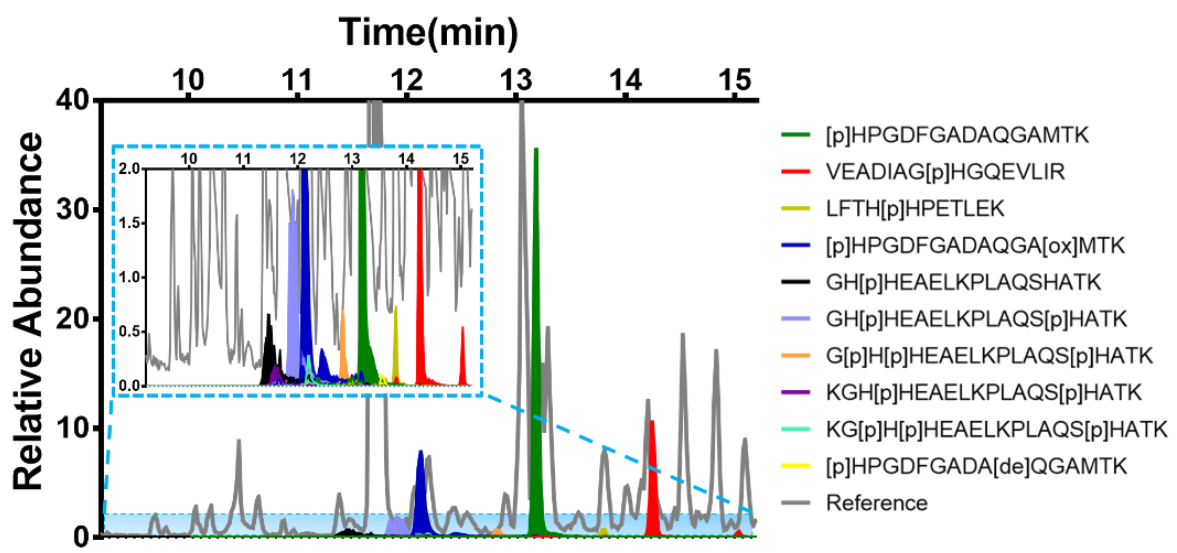

Figure 31: Extracted chromatograms highlighting histidine-phosphorylated peptides obtained from chemical N-phosphorylation of myoglobin. Each peptide indicated in colors. The background peptide signal colored in gray (reference) is the peptides from bovine serum albumin (BSA): $\beta$-casein: myoglobin (1:1:1) digest.

In Paper III, to fulfill the aim of the study, ZAP-70 phosphoprotein was obtained from stimulated Jurkat cells, a human T-lymphocytes cell line. This cell line was chosen be-cause it is known that cytoplasmic tyrosine kinases are highly and specifically activated in these cells. Gel electrophoresis was carried out using cell lysates. This technique is based on separation of macromolecules by their size and charge. Western blotting was then applied where phosphorylation sites on ZAP-70 phosphoprotein was identified using anti pTyr- antibodies. 
After localization and identification of different phosphorylation sites on ZAP-70 protein, in-gel digest was carried out. Characterization and peptide profiling of digests was completed using MALDI-TOF/MS. The schematic explanation of each step was given in Figure 32 and the details for this part was discussed in Paper III.
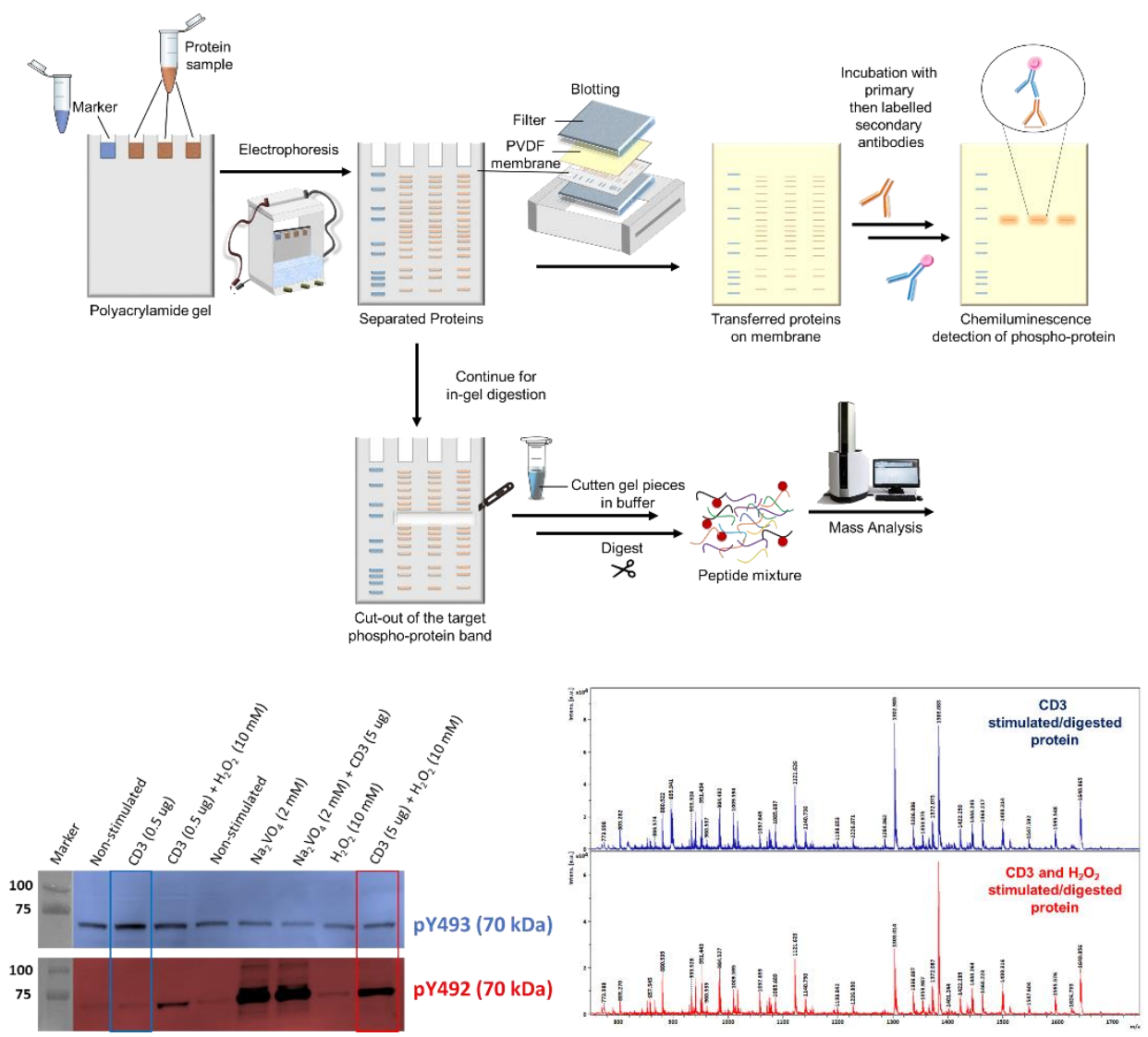

Figure 32: Schematic explanation of the workflow for the extraction of ZAP-70 phosphoprotein obtained by stimulated Jurkat T-cell line, identification by Western Blot, Immunoprecipitation, proteolytic in-gel digest and identification of peptide profile using MALDI-TOF/MS. 


\section{RESULTS AND DISCUSSION}

\section{Summary of Papers I-V}

\section{Paper I:}

\section{Urea-based imprinted polymer hosts with switchable anion preference.}

In term of oxyanions and their biological relevance, the importance of binding interaction is found related with most of the chemical and biochemical processes such as enzymatic sites, structural changes, and storage of genetic information. Earlier in the thesis mentioned that the design of synthetic receptors is particularly challenging for anions compared to cations due to being larger size, having variety of geometries, and being possible $\mathrm{pH}$ dependent. In more detail, the discrimination among phosphate and sulfate and other isoteric oxyanions requires special attention considering their competition in binding.

In Paper I, considering in-depth investigation of MIPs for simplex oxyanions, PPA (phenyl phosphonic acid), PSA (phenyl sulfonic acid), and BA (benzoic acid) were used as template in the imprinting together with functional monomer $\mathbf{1}$ as host monomer. In this study, it was aimed to answer the questions about the level of oxyanion selectivity and affinity via MIPs in either organic or aqueous media, switchable oxyanion preference using small molecule acidic or basic modifiers, and the mechanism underlying imprinting process in the presence and absence of template molecule. Considering oxyanion-oxourea complexation, the stability has been shown to increase in the order of increasing acceptor basicity, i.e., $\mathrm{PhSO}_{3}{ }^{-}<\mathrm{PhOP}(\mathrm{OH}) \mathrm{O}_{2}{ }^{-}<$ $\mathrm{PhP}(\mathrm{OH}) \mathrm{O}_{2}^{-} \leq \mathrm{PhCOO}^{-}<\mathrm{PhPO}_{3}{ }^{2-} \leq \mathrm{PhOPO}_{3}{ }^{2-}$. Evaluating the template binding studies using the neutral, monoanion and dianions of PPA, PSA, and BA via corresponding imprinted and non-imprinted polymers showed that the increase in deprotonation enhances the degree of interaction with $\mathbf{1}$. Further tests such as adsorption isotherms, switchable oxyanion selectivity, and binding in water were completed using PPA imprinted polymers. Here also, more hydrophilic crosslinking agent, PETA was introduced instead of EGDMA and the polymers synthesized with this recipe were conducted for binding selectivity tests. The investigation of imprinting mechanism was completed for fundamental understanding of the imprinting mechanism in the 
presence of template and how the presence of template is affecting the early stage in the polymerization process. The mechanistic studies were completed using differential scanning calorimetry (DSC) to investigate whether the monomer reactivity depends on template presence or randomly incorporated. Moreover, it was presented that using acidic (TFA) or basic (TEA) modifier makes possible to switch the binding of PPA or PSA. The imprinted polymer synthesized using PPA as template showed that with TEA modifier it has more binding preference for PPA; however, TFA modifier switch to binding preference to PSA (Paper I, Figure 6). In term of polymerization mechanism, it was found that the template role is to bring polymerizable units close in space and enhance the stereo- and sequence- controlled monomer incorporation (Paper I, Figure 9 and Figure 10).

\section{Paper II:}

\section{Selective enrichment of histidine phosphorylated peptides using molecularly imprinted polymers}

Even though histidine phosphorylation (pHis) is known over 50 years, the acid-labile nature of pHis hampered study of this modification. The traditional enrichment methods such as IMAC, MOAC for identification of phosphoesters (pSer, pThr, and pTyr) involve acidic conditions for enrichment of these particular phosphorylated residues. However, pHis hydrolyzes and loses its phosphate group under these conditions. Not only enrichment protocols but also the lack of affinity materials for this unique modification cause challenges for identification of pHis. For this reason, it was urge to design and develop efficient tools where pHis can be identified and enriched from complex mixture under mild enrichment conditions where preserving the modification.

Paper II describes a method for selective enrichment of pHis peptides using three different imprinted polymers (MIP1-3) under non-acidic conditions. The pHis MIPs were synthesized using a stable analogue of phosphohistidine (Fmoc-pTza-OEt) template together with functional monomer 1. Their non-imprinted polymers (NIP1-3) were synthesized under same condition by omitting template molecule. MIP1 and MIP2 are high-crosslinked polymers synthesized using different counterions, PMP and TBA.OH, respectively. MIP3 is mesoporous imprinted microspheres synthesized in porous silica microparticles using the same recipe for MIP2. The polymers were tested with peptide mixture prepared in different $\mathrm{MeCN} / \mathrm{H}_{2} \mathrm{O}$ ratios with $0.1 \%$ TEA (triethlyamine, basic modifier). First test was completed using MIP1 and NIP1 to evaluate their affinity towards template molecule, pTza. Further short peptide (VpHI, 
VpSI, VHI, and VEI) mixture was treated with MIP1 and NIP1. The polymer displayed promising affinity towards pHis peptide, $\mathrm{VpHI}$, in $95 \% \mathrm{MeCN}+0.1$ TEA. Due to the interfering affinity for pSer modification, the phosphoesters treated with alkaline conditions via $\beta$-elimination where VpSI and VpTI dephosphorylated. This approach proof the strong retention of $\mathrm{VpHI}$ peptide without interfered affinity. Then MIP1 recipe was changed and MIP2 was synthesized. MIP2 was able to enrich VpHI peptide in the presence of VpSI and VpTI short peptides spiked in tryptic digest of BSA without requiring any $\beta$-elimination. However, when MIP2 was tested for the enrichment of chemically phosphorylated myoglobin pHis-peptides mixed with equimolar BSA and $\beta$-casein digests, it showed poor selectivity. Since MIP2 is highcrosslinked polymer with a wide distribution of pores, ranging from micro-pores with limited larger peptides accessibility to meso- or macro-pores, MIP3 was synthesized resulting mesoporous microspheres featured a controlled and narrow pore size distribution. MIP3 showed improved selectivity and specificity for the enrichment of larger-sized pHis-peptides from complex mixture. Moreover, MIP3 increased the abundance of pHis peptides compared to pSer- and pThr- peptides.

Overall, the MIP-based protocol reported in Paper II exhibited alternative way for the enrichment of pHis peptides from complex mixture that is compatible with preserving $\mathrm{pHis}$ modification and facilitating promising strategy to explore this PTM.

\section{Paper III:}

\section{MIP-Binder for sequence specific phosphopeptide capture of ZAP-70 regu- latory motifs.}

Tyrosine phosphorylation, pTyr is a sub-stoichiometric modification that occurs in low abundance where detection and identification can be done using anti pTyr-antibodies, or metal oxide-based affinity materials. Even though immunoaffinity enrichment coupled to MS based identification and quantification exhibit promising approach translating the knowledge to clinical applications, there is still need for simple and robust techniques capable of separating or sensing this particular PTM for the development of effective diagnostics in early stage and treatment of diseases. Apart from developing a generic fractionation tool that is capable of capturing all phosphorylated residues such as pTyr, pSer, or pThr over non-phosphorylated peptides, the other levels of selectivity are equally critical such as regio- or stoichiometric- selectivity. Therefore, synthetic receptors prepared using imprinting approach that can recognize amino acid sequence around different phosphorylation sites would help in diagnostic applications in the case of biomarker discovery. 
Paper III describes a concept where MIPs can capture phosphopeptides in a sequence specific manner in complex media. In this paper, imprinted polymers were prepared for targeting on phosphorylation of epitope tripeptides of ZAP-70 (zeta-associated 70$\mathrm{kDa}$ protein) which has the following peptide sequence of amino acids, ALGADDSYYTAR (485-496) in the kinase domain. ZAP-70 is a positive prognosticator for the disease chronic lymphatic leukemia, CLL. The phosphorylation of Y492 or Y493 in the kinase domain is identified as regulators for the disease. Regarding to the target pY-ZAP-70, four imprinted polymers were prepared using the synthesized Nand C- protected templates: YpY MIP (targeting pY493), pYY MIP (targeting pY492), pYpY MIP (targeting pY492pY493), and YY MIP as control for the following sequence, ALGADDSY ${ }^{492} \mathbf{Y}^{493} \mathrm{TAR}$.

The synthesized polymers were firstly tested with the mixture of model peptides: GADDSYYTAR, GADDSYpYTAR, GADDSpYYTAR, and GADDSpYpYTAR prepared at different $\mathrm{MeCN} / \mathrm{H}_{2} \mathrm{O}$ ratio buffered with $0.1 \%$ acidic and basic modifiers and aqueous buffers at different $\mathrm{pH}$. The sequence specific $\mathrm{pY}$-targeting polymers displayed promising affinity towards their corresponding targets when tested in $90 \%$ $\mathrm{MeCN}+0.1 \%$ TFA (trifluoroacetic acid) and aqueous buffer, $\mathrm{pH}=7.4$. Next, the model decapeptides were spiked with 12-protein digests at different spiking level. Then, the performance of sequence specific receptors were tested to clear their ability to enrich target p-peptide from complex mixture. The abundance of target peptide in the elution fraction over nontarget and nonphosphorylated peptides using receptors exhibited promising site- and stoichiometric- selective discrimination compared to the abundance of peptides in pre-enrichment fraction (Paper III, Figure 3 and Figure S5). Having determined that these sequence specific binders are able to enrich their particular target from complex mixture, further study on enrichment of dodeca- peptides via digested ZAP-70 phosphoproteins that were extracted from stimulated Jurkat T-cells. In this particular experiment, Jurkat cells were stimulated with different regulators and their combinations. It was found that non-stimulated lysate showed already constitutive hyperphosphorylation of Y493 pTyr site and the other regulators showed variety of phosphorylation profiles (Paper III, Figure 4). The experiments on the enrichment of phosphorylated peptides obtained from stimulated lysates are still undergoing. Nevertheless, the results presented in the enrichment of target p-peptide from complex mixture showed the potential use of imprinting approach to design sequence specific receptors, which are capable of enrichment of phosphorylated residues in other levels of selectivity. 


\section{Paper IV:}

\section{High salt compatible oxyanion receptors by dual ion imprinting.}

Studies showed that using TBA transfer agent together with target anion where functional monomer $\mathbf{1}$ used in the polymerization process promote to generate high affinity binding pocket for recognition. For instance the association constant for PPA $\bullet$ TBA guest and 1 host found as $K_{a}=7005 \pm 985$. However, these synthetic receptors for anion recognition is usually selective in aprotic polar solvent but weaken and become highly competitive in media presented high ionic strength. For this reason, introducing another host compatible with naturally most common counterion of guest could enhance the affinities targeting not only anion but also counterion.

Paper IV describes that using crownether which, is macrocyclic host where it can generate 1:1 host:guest complexation in the presence of metal ion, together with anion guest and $\mathbf{1}$ host in the pre-polymerization can feature strong cooperativity for the recognition of target anions in more amphiphilic nature. In the study, PPA $\cdot \mathrm{Na}$ template was imprinted in the presence of $\mathbf{1}$ and 18-crown-6 (18C6) and their binding profiles in buffer prepared at different $\mathrm{pH}$ was compared. Moreover, the titration of 18C6-Na•PPA complex with 1 resulted an associated constant, $K_{a}=11181 \pm 1655$ which is significantly higher than titrating PPA•TBA complex with $\mathbf{1}$ (Paper IV, Figure S1, Table 1). Moreover, the testing binding capacities and association constant in the absence and presence of NaCI solution revealed (Paper IV, Figure 4) that combining macrocyclic cation host with neutral urea-based monomer in the anion recognition effectively enhance the affinity and capacity in the recognition of anion in buffers.

\section{Paper V:}

\section{Imprinted Src-SH2 domain mimicking: Targeting pYEEI sequence.}

Earlier, the discovery of Src homology 2, SH2 domain was described. SH2 domains are known as phosphorylation- especially pTyr containing peptides and sequence specific- dependent variants that play critical role in receptor signaling and signal transduction pathways. SH2 domain is a part of Src family kinases that are modular signaling domains. There are different members were identified such as Src, Lck, Fyn, Lyn, and more. These subgroups have different pTyr peptide specificity related with its motif and sequence. For instance, Src and Lck prefer to bind the motif of pTyrhydrophilic-hydrophilic-Ile/Pro (Ile: Isoleucine, Pro: Proline). Src-SH2 domain is specifically bind the sequence of pY-E-E-I, pTyr-Glu-Glu-Ile (Glu: Glutamic acid) peptide with higher affinity in $\mathrm{nM}$ (nanomolar) range (Paper V, Scheme 1). 
Paper V described the synthesis of pYEEI sequence specific affinity-capture-material, which can mimic the binding of Src-SH2 domain. In this paper, three different polymerization formats that are high-crosslinked, high-dilution, and mesoporous microspheres were used in the presence of N- and C- protected Fmoc-pYEEI-OEt and Fmoc-YEEI-OEt templates using 1:2 and 1:4 stoichiometric imprinting. The polymers were tested with the mixture of model peptides, EPQpYEEIPIYL and EPQYEEIPIYL, prepared in $95 \% \mathrm{MeCN}+0.1 \%$ TFA or TEA and aqueous buffers at different $\mathrm{pH}$. The promising phospho-template imprinted ones for each polymerization format was further carried out for enrichment and sequence specificity tests. The sequence specificity of imprinted polymers were tested with the mixture of following phosphorylated and non-phosphorylated peptides: EPQYEEIPIYL, EPQpYEEIPIYL，DRVYIHPF，DRVpSIHPF，DRVpYIHPF，GADDSYYTAR, GADDSpYYTAR, GADDSpYpYTAR, and their binding preference compared with their corresponding non-imprinted polymers. The mesoporous microsphere synthesized with 1:2 stoichiometry showed the highest selectivity with a clean extraction towards target p-peptide (Paper V, Figure 4, 5, and 6). Moreover, two model peptides were spiked with $\beta$-casein digest at 1:25 spiking level. The peptide profile in each fraction was monitored using MALDI-TOF/MS. The comparison between elution fractions of pY-MIPs and NIPs confirmed the affinity of pY-MIPs towards target peptide. This study is undergoing and it has great potential on targeted phosphoproteomics, especially considering the development of highly specific kinase inhibitors based on anticancer agents. 


\section{CONCLUDING REMARKS AND FUTURE OUTLOOK}

The research presented in this thesis is focused on designing, synthesizing and developing amino acid side chain- and sequence- specific molecularly imprint-ed polymer receptors targeting protein phosphorylation.

Protein phosphorylation is identified as a reversible PTM that play a critical role in many regulatory mechanisms of the human genome. The process is carried out by protein kinases that transfer phosphate group from ATP to amino acid residues and phosphorylate them. Studies aiming at understanding the fundamental mechanisms of cell signaling are strongly associated cancer medicine. In fact, most protein kinases are related to oncogenesis beside their normal role in basic biological processes such as cell growth and apoptosis. Not only specific mutations or dysregulation on kinases are associated with human malignancies, but also genetically inherited variants of particular kinases is critical for the initiation, promotion and progression of a disease. Therefore, it was inevitable that kinase inhibitors-based drugs have become significantly predominant for the treatment of cancer and other diseases. Among the number of approved kinase-inhibitors, some showed resistance and failure due to kinase mutations. Except for leading to resistance to treatment, most kinase inhibitors are targeting highly conserved ATP-binding pocket, and therefore their affinity and selectivity for a particular kinase and its corresponding disease relevance is not very clear. For this reason, proteomics-derived biomarkers are used to provide a critical disease signature, offering in turn an important guide for further treatment. Biomarkers can be diagnostic, prognostic, and predictive tools. For instance, prostate-specific antigen (PSA) is used for the detection of prostate cancer; however, its poor selectivity fails to discriminate between prostate cancer and other relative pathologies. For this reason, high-throughput genomic, proteomic and metabolomics technologies have been used to find more precise predictive biomarkers for clinical applications. In this context an urgent need for robust affinity techniques has emerged that can provide easy-to-use and reliable biomarker identification and detection.

Protein phosphorylation analysis using mass spectrometry and applications of enrichment techniques such as IMAC and MOAC in particular are commonly restricted to generic enrichment of phosphorylated residues. Here molecularly imprinted 
polymers offers a new dimension in offering the ability to tailor the enrichments towards specific amino acid modifications or peptide sequences. This may reveal new previously unknown phosphorylation sites, or offer an important tool for targeted phosphoproteomics.

Particularly in this thesis, side chain- and sequence- specific imprinted polymers were introduced. We show, for the first time, that sequence specific binders are capable of specific enrichment of the targeted pTyr peptides from a complex biological sample. Moreover, addressing the well-known challenges associated with enrichment and identification of labile phosphorylations, carefully engineered pTza-MIPs are capable of specifically enriching pHis peptides placing MIPs on the map of enrichment techniques for this particular acid-labile phosphorylation.

Despite the above advances, further optimization and validations are required for efficient use of MIPs in clinical phosphoproteomic. For instance, the following points should be further investigated: i) suppression of MIP heterogeneity to boost binding capacity and reduce nonspecific binding, ii) further MIP optimization to enhance compatibility with aqueous media and in turn enrichment of targets from native biological sample iii) further validation of the enrichment protocol and parameter optimization e.g. the sorbent/sample volume ratio, solvent system and modifiers, iv) generating selective binding pockets in nano-sized MIPs for biological applications.

Overcoming possible limitations in the field of imprinting would offer selective, robust, and low production cost synthetic receptors compatible with robust cancer theranostics platforms. The results presented in this thesis suggest that this goal is realistic and that it can be achieved within the foreseeable future. 


\section{ACKNOWLEDGEMENTS}

I would like to say that this thesis would not be possible without the help, support and contributions from the people around me. Particularly I would like to express my gratitude to these people below.

Börje Sellergren, my supervisor for giving this great opportunity to pursue my Ph.D., his continuous support, his immense advice and encouragement. Particularly, for his always enthusiastic and optimistic attitude, and his motivation through research.

Maria Magdalena Stollenwerk, my co-supervisor for her constant support, advice and encouragement, and for her help in cell experiments.

Tautgirdas Ruzgas, my Ph.D. examiner for his assistance through all courses and taking care of any administrative problem.

To collaborators in BIOCAPTURE project, Ole Nørregaard Jensen (Southern Denmark University) for his help, advice and insightful conversations about proteomics and mass spectrometry, Ian Alan Nicholls and Gustaf Olsson (Linnaeus University) for their support, advice, and motivation on molecular dynamic simulations, Knut Rurack for his help and advice in fluorescent materials. To industrial partners, Justin Jordaan, Stoyan Stoychev, and Isak Gerber (ReSyn Biosciences) and Ian Pike (Proteome Sciences) for their collaboration, support and team work to test materials and initiation on commercialization.

Sudhirkumar Arjun Shinde for his help in the beginning of project and his continuous support and advice during my Ph.D., sharing his knowledge about MIPs, phosphoproteomics and endless conversations about science and life.

Celina Wierzbicka for her help in the beginning of project and sharing her knowledge about MIPs and phosphoproteomics.

Ignacio Arribas Diez for his continuous help on phosphoproteomics and mass spectrometry and his endless effort and time for scientific contributions.

Biocapture folks, Liliia Mavliutova, Rashmi Mahajan, Tuğrul Cem Bıçak, Ignacio Arribas Diez, Chau Huynh, Evgeniia Kislenko, Sam Burnage, Nicholas McKitterick, Julie van Coilie, and Rebecca Nason for sharing your friendship and knowledge. Our moments in Biocapture journey were unforgettable. We should cherish and be proud of ourselves. 
All the present and former members of Biofilms Research Center for Biointerfaces group for friendship, discussions, birthdays, and fikas! It was an amazing journey to have you all around. Liliia Mavliutova, Radvile Zubryte, Michele Addosso, Razieh Sahraei, Mona Rouhi, Sing Yee Yeung, Giuliana Grasso, Vivek Chaturverdi, Aura Rocio Hernandez, and Qianjin Li you have no idea how much I appreciate for great start in Malmö University. Thanks Skaidre Jankovskaja, Ekaterina Bogdanova Zevak, Emelie Josefina Nilsson, Hannah Boyd, Dainius Jakubauskas, Sarah Wilde, Maxim Morin, Yubexi Marcano, Michal Szczepanczyk, Abdullah Ali, Nicolo Paracini, Rita Del Giudice, Ramunas Digaitis, Atefeh Shafaat, Mina Fazilati, Stephanie Tassler, Enamul Mojumdar, Sebastian Björklund, Javier Sotres, many others that I did not mention here for creating nice atmosphere both in the office and laboratory.

To Sellegren's Research current group members, Liliia Mavliutova, Thomas Janssens, Marco Dattilo, Elena Verduci, Yulia Sergeeva, Rahma Abouhany, George Lasisi, and Sara Sigurdardottir.

Vitaly Kolcherbitov thank you for your help in DSC.

Marite Cardenas thank you for an amazing summer school, our scientific discussion and letting me to do experiments in your research lab.

Juan Fransisco Gonzales thank you for your help with MALDI.

Peter Falkman thank you for help with SEM, NMR, and all technical problems.

Special thanks to Liliia Mavliutova for being always there and sharing all ups and downs.

Alper Yılmaz, Sinem Evli, Müge Yücel, Ekin Meşe, and Hakan Gül, thank you all for your friendship.

The financial support from ITN project BIOCAPTURE - the European Union's Horizon 2020 research and innovation programme under the Marie Skłodowska-Curie grant agreement No 722171 and Malmö University, Biofilms Research Center for Bio-interfaces (BRCB).

I would like to thank three very important people in my career, first Onur Parlak for always being there when I need any help about science and life, and my supervisors during my master study, Mustafa Muammer Demir and Umit Hakan Yıldız who initiated and helped to develop my passion about science.

Finally to my parents for their endless love and support. 


\section{REFERENCES}

1. Csermely, P., Palotai, R. \& Nussinov, R. Induced fit, conformational selection and independent dynamic segments: An extended view of binding events. Trends Biochem Sci (2010).

2. Part II: Molecular Recognition in Protein Assay. in Nano-Inspired Biosensors for Protein Assay with Clinical Applications (2019).

3. Cragg, P. J. Supramolecular chemistry: From biological inspiration to biomedical applications. Supramolecular Chemistry: From Biological Inspiration to Biomedical Applications (2010).

4. Lehn, J. -M. Perspectives in Supramolecular Chemistry-From Molecular Recognition towards Molecular Information Processing and Self-Organization. Angewandte Chemie International Edition in English (1990).

5. Aida, T., Meijer, E. W. \& Stupp, S. I. Functional supramolecular polymers. Science (2012).

6. Chierotti, M. R. \& Gobetto, R. Supramolecular Chemistry: From Molecules to Nanomaterials. Supramol Chem From Mol to Nanomater (2012).

7. The Chemistry of Molecular Recognition - Host Molecules and Guest Molecules. in Supramolecular Chemistry - Fundamentals and Applications (2006).

8. Ogoshi, T. \& Yamagishi, T. A. CHAPTER 1: Historical background of macrocyclic compounds. in Monographs in Supramolecular Chemistry (2016).

9. Cragg, P. J. \& Vahora, R. Crown and Lariat Ethers. in Supramolecular Chemistry (2012).

10. Barnes, J. C. \& Mirkin, C. A. Profile of Jean-Pierre Sauvage, Sir J. Fraser Stoddart, and Bernard L. Feringa, 2016 nobel laureates in chemistry. Proc Natl Acad Sci U S A (2017).

11. Sellergren, B. Molecularly Imprinted Polymers: Man-Made mimics of antibodies and their applications. Journal of Chemical Information and Modeling (2013).

12. Wulff, G. Molecular Imprinting in Cross-Linked Materials with the Aid of Molecular Templates- A Way towards Artificial Antibodies. Angewandte Chemie International Edition in English (1995).

13. Wulff, G. The role of binding-site interactions in the molecular imprinting of polymers. Trends in Biotechnology (1993).

14. Sellergren, B. \& Shea, K. J. Influence of polymer morphology on the ability of imprinted network polymers to resolve enantiomers. J Chromatogr A (1993).

15. Sellergren, B., Lepist, M. \& Mosbachö, K. Highly enantioselective and substrate-selective polymers obtained by molecular imprinting utilizing noncovalent interactions. NMR and chromatographic studies on the nature of recognition. J Am Chem Soc (1988).

16. Arshady, R. \& Mosbach, K. Synthesis of substrate-selective polymers by host-guest polymerization. Die Makromol Chemie (1981).

17. Wulff, G. Fourty years of molecular imprinting in synthetic polymers: Origin, features and perspectives. Microchimica Acta (2013).

18. Andersson, L., Sellergren, B. \& Mosbach, K. Imprinting of amino acid derivatives in macroporous polymers. Tetrahedron Lett (1984). 
19. Wulff, G., Oberkobusch, D. \& Minárik, M. Enzyme-analogue built polymers, 18 chiral cavities in polymer layers coated on wide-pore silica. React Polym Ion Exch Sorbents (1985).

20. Ward, I. M. Polymers: Chemistry and Physics of Modern Materials, 3rd edition, by J.M.G. Cowie and V. Arrighi. Contemp Phys (2009).

21. Li, W. H., Hamielec, A. E. \& Crowe, C. M. Kinetics of the free-radical copolymerization of methyl methacrylate/ethylene glycol dimethacrylate: 1. Experimental investigation. Polymer (Guildf) (1989).

22. Ansell, R. J. Characterization of the binding properties of molecularly imprinted polymers. Adv Biochem Eng Biotechnol (2015).

23. Nicholls, I. A. et al. Can we rationally design molecularly imprinted polymers? in Analytica Chimica Acta (2001).

24. Nicholls, I. A. Towards the rational design of molecularly imprinted polymers. in Journal of Molecular Recognition (1998).

25. Wulff, G. \& Knorr, K. Stoichiometric noncovalent interaction in molecular imprinting. Bioseparation (2001).

26. Wulff, G. \& Biffis, A. Chapter 4 Molecular imprinting with covalent or stoichiometric noncovalent interactions. in Techniques and Instrumentation in Analytical Chemistry (2001).

27. Sellergren, B. Molecular imprinting by noncovalent interactions. Enantioselectivity and binding capacity of polymers prepared under conditions favoring the formation of template complexes. Die Makromol Chemie (1989).

28. Maeda, M. \& Bartsch, R. A. Molecular and Ionic Recognition with Imprinted Polymers: A Brief Overview. ACS Symposium Series (1998).

29. Haupt, K. Noncovalent Molecular Imprinting of a Synthetic Polymer with the Herbicide 2,4Dichlorophenoxyacetic Acid in the Presence of Polar Protic Solvents. ACS Symp Ser (1998).

30. Yan, M. \& Ramström, O. Molecularly imprinted materials: Science and technology. Molecularly Imprinted Materials: Science and Technology (2004).

31. Puoci, F. et al. Molecularly imprinted solid phase extraction for the selective HPLC determination of $\alpha$-tocopherol in bay leaves. Anal Chim Acta (2007).

32. Pichon, V. \& Chapuis-Hugon, F. Role of molecularly imprinted polymers for selective determination of environmental pollutants-A review. Analytica Chimica Acta (2008).

33. Bossi, A., Bonini, F., Turner, A. P. F. \& Piletsky, S. A. Molecularly imprinted polymers for the recognition of proteins: The state of the art. Biosensors and Bioelectronics (2007).

34. Shi, Y. et al. Uniform molecularly imprinted poly(methacrylic acid) nanospheres prepared by precipitation polymerization: The control of particle features suitable for sustained release of gatifloxacin. J Mater Chem (2012).

35. Matsui, J., Miyoshi, Y. \& Takeuchi, T. Fluoro-functionalized Molecularly Imprinted Polymers Selective for Herbicides. Chem Lett (1995).

36. Kugimiya, A., Matsui, J. \& Takeuchi, T. Sialic acid-imprinted polymers using noncovalent interactions. Mater Sci Eng C (1997).

37. Breton, F., Delépée, R., Jégourel, D., Deville-Bonne, D. \& Agrofoglio, L. A. Selective adenosine-5'-monophosphate uptake by water-compatible molecularly imprinted polymer. Anal Chim Acta (2008).

38. Krstulja, A. et al. Evaluation of molecularly imprinted polymers using 2',3',5'-tri-Oacyluridines as templates for pyrimidine nucleoside recognition. Anal Bioanal Chem (2014). 
39. Spivak, D. A. \& Shea, K. J. Investigation into the scope and limitations of molecular imprinting with DNA molecules. in Analytica Chimica Acta (2001).

40. Yano, K. et al. Molecularly imprinted polymers which mimic multiple hydrogen bonds between nucleotide bases. Anal Chim Acta (1998).

41. Sellergren, B. Polymer- and template-related factors influencing the efficiency in molecularly imprinted solid-phase extractions. TrAC - Trends Anal Chem (1999).

42. Navarro-Villoslada, F., San Vicente, B. \& Moreno-Bondi, M. C. Application of multivariate analysis to the screening of molecularly imprinted polymers for bisphenol a. Anal Chim Acta (2004). 43. Mollnelli, A. et al. Analyzing the mechanisms of selectivity in biomimetic self-assemblies via IR and NMR spectroscopy of prepolymerization solutions and molecular dynamics simulations. Anal Chem (2005).

44. Shi, X., Wu, A., Qu, G., Li, R. \& Zhang, D. Development and characterisation of molecularly imprinted polymers based on methacrylic acid for selective recognition of drugs. Biomaterials (2007).

45. Cormack, P. A. G. \& Elorza, A. Z. Molecularly imprinted polymers: Synthesis and characterisation. Journal of Chromatography B: Analytical Technologies in the Biomedical and Life Sciences (2004).

46. Crick, F. Central dogma of molecular biology. Nature (1970).

47. Jensen, O. N. Interpreting the protein language using proteomics. Nature Reviews Molecular Cell Biology (2006).

48. Blackstock, W. P. \& Weir, M. P. Proteomics: Quantitative and physical mapping of cellular proteins. Trends in Biotechnology (1999).

49. Jensen, O. N. Modification-specific proteomics: Characterization of post-translational modifications by mass spectrometry. Current Opinion in Chemical Biology (2004).

50. Aebersold, R. et al. How many human proteoforms are there? Nature Chemical Biology (2018).

51. Smith, L. M. \& Kelleher, N. L. Proteoform: A single term describing protein complexity. Nature Methods (2013).

52. Humphrey, S. J., James, D. E. \& Mann, M. Protein Phosphorylation: A Major Switch Mechanism for Metabolic Regulation. Trends in Endocrinology and Metabolism (2015).

53. Taylor, S. S. \& Kornev, A. P. Protein kinases: Evolution of dynamic regulatory proteins. Trends in Biochemical Sciences (2011).

54. Cohen, P. The role of protein phosphorylation in human health and disease. . Eur J Biochem (2001).

55. Aschner, Y. \& Downey, G. P. The importance of tyrosine phosphorylation control of cellular signaling pathways in respiratory disease: $\mathrm{pY}$ and pY not. American Journal of Respiratory Cell and Molecular Biology (2018).

56. Mann, M. et al. Analysis of protein phosphorylation using mass spectrometry: Deciphering the phosphoproteome. Trends in Biotechnology (2002).

57. Craig Venter, J. et al. The sequence of the human genome. Science (80- ) (2001).

58. Hunter, T. \& Sefton, B. M. Transforming gene product of Rous sarcoma virus phosphorylates tyrosine. Proc Natl Acad Sci U S A (1980).

59. Olsen, J. V. et al. Global, In Vivo, and Site-Specific Phosphorylation Dynamics in Signaling Networks. Cell (2006). 
60. Attwood, P. V., Piggott, M. J., Zu, X. L. \& Besant, P. G. Focus on phosphohistidine. in Amino Acids (2007).

61. Besant, P., Attwood, P. \& Piggott, M. Focus on Phosphoarginine and Phospholysine. Curr Protein Pept Sci (2009).

62. Attwood, P. V., Besant, P. G. \& Piggott, M. J. Focus on phosphoaspartate and phosphoglutamate. Amino Acids (2011).

63. Marmelstein, A. M., Moreno, J. \& Fiedler, D. Chemical Approaches to Studying Labile Amino Acid Phosphorylation. Topics in Current Chemistry (2017).

64. Yu, J. S. From discovery of tyrosine phosphorylation to targeted cancer therapies: The 2018 Tang Prize in Biopharmaceutical Science. Biomedical Journal (2019).

65. Sörby, M. \& Östman, A. Protein-tyrosine phosphatase-mediated decrease of epidermal growth factor and platelet-derived growth factor receptor tyrosine phosphorylation in high cell density cultures. J Biol Chem (1996).

66. Hunter, T. Tyrosine phosphorylation: thirty years and counting. Current Opinion in Cell Biology (2009).

67. Wagner, M. J., Stacey, M. M., Liu, B. A. \& Pawson, T. Molecular mechanisms of SH2- and PTB-Domain-containing proteins in receptor tyrosine kinase signaling. Cold Spring Harb Perspect Biol (2013).

68. Avruch, J. et al. Ras activation of the Raf kinase: Tyrosine kinase recruitment of the MAP kinase cascade. Recent Progress in Hormone Research (2001).

69. Cuevas, B. D. et al. Tyrosine Phosphorylation of p85 Relieves Its Inhibitory Activity on Phosphatidylinositol 3-Kinase. J Biol Chem (2001).

70. Kapeller, R. \& Cantley, L. C. Phosphatidylinositol 3-kinase. BioEssays (1994).

71. Yamaoka, K. et al. The Janus kinases (Jaks). Genome Biology (2004).

72. Rane, S. G. \& Reddy, E. P. Janus kinases: Components of multiple signaling pathways. Oncogene (2000).

73. Wardenburg, J. B. et al. Phosphorylation of SLP-76 by the ZAP-70 protein-tyrosine kinase is required for T-cell receptor function. J Biol Chem (1996).

74. Di Bartolo, V. et al. Tyrosine 319, a newly identified phosphorylation site of ZAP-70, plays a critical role in T cell antigen receptor signaling. J Biol Chem (1999).

75. Wang, H. et al. ZAP-70: an essential kinase in T-cell signaling. Cold Spring Harbor perspectives in biology (2010).

76. Marasco, M. \& Carlomagno, T. Specificity and regulation of phosphotyrosine signaling through SH2 domains. J Struct Biol X (2020).

77. Park, M. J. et al. SH2 Domains Serve as Lipid-Binding Modules for pTyr-Signaling Proteins. Mol Cell (2016).

78. Hunter, T. Phosphotyrosine - a new protein modification. Trends Biochem Sci (1982).

79. Attwood, P. V. Histidine kinases from bacteria to humans. Biochem Soc Trans (2013).

80. Kee, J. M. \& Muir, T. W. Chasing phosphohistidine, an elusive sibling in the phosphoamino acid family. ACS Chemical Biology (2012).

81. Adam, K. \& Hunter, T. Histidine kinases and the missing phosphoproteome from prokaryotes to eukaryotes. Laboratory Investigation (2018).

82. Hultquist, D. E. The preparation and characterization of phosphorylated derivatives of histidine. BBA - Bioenerg (1968). 
83. Matthews, H. R. Protein kinases and phosphatases that act on histidine, lysine, or arginine residues in eukaryotic proteins: A possible regulator of the mitogen-activated protein kinase cascade. Pharmacology and Therapeutics (1995).

84. Schaller, G. E., Shiu, S. H. \& Armitage, J. P. Two-component systems and their co-option for eukaryotic signal transduction. Current Biology (2011).

85. Puttick, J., Baker, E. N. \& Delbaere, L. T. J. Histidine phosphorylation in biological systems. Biochimica et Biophysica Acta - Proteins and Proteomics (2008).

86. Adam, K., Ning, J., Reina, J. \& Hunter, T. Nme/nm23/ndpk and histidine phosphorylation. Int J Mol Sci (2020).

87. Kee, J. M., Oslund, R. C., Perlman, D. H. \& Muir, T. W. A pan-specific antibody for direct detection of protein histidine phosphorylation. Nat Chem Biol (2013).

88. Fuhs, S. R. et al. Monoclonal 1- and 3-Phosphohistidine Antibodies: New Tools to Study Histidine Phosphorylation. Cell (2015).

89. Harsha, H. C. \& Pandey, A. Phosphoproteomics in cancer. Molecular Oncology (2010).

90. Martin, G. S. Cell signaling and cancer. in Cancer Cell (2003).

91. Alexander, P. B. \& Wang, X. F. Resistance to receptor tyrosine kinase inhibition in cancer: molecular mechanisms and therapeutic strategies. Frontiers of Medicine (2015).

92. Dankner, M., Rose, A. A. N., Rajkumar, S., Siegel, P. M. \& Watson, I. R. Classifying BRAF alterations in cancer: New rational therapeutic strategies for actionable mutations. Oncogene (2018). 93. Du, Z. \& Lovly, C. M. Mechanisms of receptor tyrosine kinase activation in cancer. Molecular Cancer (2018).

94. Lemmon, M. A. \& Schlessinger, J. Cell signaling by receptor tyrosine kinases. Cell (2010).

95. McLean, G. W. et al. The role of focal-adhesion kinase in cancer - A new therapeutic opportunity. Nature Reviews Cancer (2005).

96. Zhao, X. \& Guan, J. L. Focal adhesion kinase and its signaling pathways in cell migration and angiogenesis. Advanced Drug Delivery Reviews (2011).

97. Mitra, S. K., Hanson, D. A. \& Schlaepfer, D. D. Focal adhesion kinase: In command and control of cell motility. Nature Reviews Molecular Cell Biology (2005).

98. Steelman, L. S. et al. JAK/STAT, Raf/MEK/ERK, PI3K/Akt and BCR-ABL in cell cycle progression and leukemogenesis. Leukemia (2004).

99. Brahmkhatri, V. P., Prasanna, C. \& Atreya, H. S. Insulin-like growth factor system in cancer: Novel targeted therapies. BioMed Research International (2015).

100. Flight, M. H. Neurodegenerative diseases: New kinase targets for Alzheimer's disease. Nature Reviews Drug Discovery (2013).

101. Banks, A. S. et al. An ERK/Cdk5 axis controls the diabetogenic actions of PPAR $\gamma$. Nature (2015).

102. Kumar, R., Singh, V. P. \& Baker, K. M. Kinase inhibitors for cardiovascular disease. Journal of Molecular and Cellular Cardiology (2007).

103. Moon, S. Y., Kim, K. D., Yoo, J., Lee, J. H. \& Hwangbo, C. Phytochemicals targeting JAKSTAT pathways in inflammatory bowel disease: Insights from animal models. Molecules (2021).

104. Bhullar, K. S. et al. Kinase-targeted cancer therapies: Progress, challenges and future directions. Molecular Cancer (2018).

105. Yamaoka, T., Kusumoto, S., Ando, K., Ohba, M. \& Ohmori, T. Receptor tyrosine kinasetargeted cancer therapy. International Journal of Molecular Sciences (2018). 
106. Levitzki, A. \& Klein, S. My journey from tyrosine phosphorylation inhibitors to targeted immune therapy as strategies to combat cancer. Proc Natl Acad Sci U S A (2019).

107. Cicenas, J., Zalyte, E., Bairoch, A. \& Gaudet, P. Kinases and cancer. Cancers (2018).

108. Ludwig, J. A. \& Weinstein, J. N. Biomarkers in cancer staging, prognosis and treatment selection. Nature Reviews Cancer (2005).

109. Liang, Y., Zhang, T. \& Zhang, J. Natural tyrosine kinase inhibitors acting on the epidermal growth factor receptor: Their relevance for cancer therapy. Pharmacological Research (2020).

110. McLafferty, F. W. A century of progress in molecular mass spectrometry. Annu Rev Anal Chem (2011).

111. Iwamoto, N. \& Shimada, T. Recent advances in mass spectrometry-based approaches for proteomics and biologics: Great contribution for developing therapeutic antibodies. Pharmacology and Therapeutics (2018).

112. Kelleher, N. L. et al. Top down versus bottom up protein characterization by tandem highresolution mass spectrometry. J Am Chem Soc (1999).

113. Maleknia, S. D. \& Johnson, R. Mass Spectrometry of Amino Acids and Proteins. in Amino Acids, Peptides and Proteins in Organic Chemistry (2011).

114. Wu, H. Y. \& Liao, P. C. Analysis of protein phosphorylation using mass spectrometry. Chang Gung Medical Journal (2008).

115. Thingholm, T. E., Jensen, O. N. \& Larsen, M. R. Analytical strategies for phosphoproteomics. Proteomics (2009).

116. Neville, D. C. A. et al. Evidence for phosphorylation of serine 753 in CFTR using a novel metal- ion affinity resin and matrix-assisted laser desorption mass spectrometry. Protein Sci (1997). 117. Posewitz, M. C. \& Tempst, P. Immobilized gallium(III) affinity chromatography of phosphopeptides. Anal Chem (1999).

118. Riley, N. M. \& Coon, J. J. Phosphoproteomics in the Age of Rapid and Deep Proteome Profiling. Anal Chem (2016).

119. Zhou, H. et al. Robust phosphoproteome enrichment using monodisperse microsphere-based immobilized titanium (IV) ion affinity chromatography. Nat Protoc (2013).

120. Feng, S. et al. Immobilized zirconium ion affinity chromatography for specific enrichment of phosphopeptides in phosphoproteome analysis. Mol Cell Proteomics (2007).

121. Low, T. Y. et al. Widening the bottleneck of phosphoproteomics: Evolving strategies for phosphopeptide enrichment. Mass Spect. Rev. (2021).

122. Napper, S. et al. Selective extraction and characterization of a histidine-phosphorylated peptide using immobilized copper(II) ion affinity chromatography and matrix-assisted laser desorption/ionization time-of-flight mass spectrometry. Anal Chem (2003).

123. Li, X. S., Yuan, B. F. \& Feng, Y. Q. Recent advances in phosphopeptide enrichment: Strategies and techniques. TrAC - Trends in Analytical Chemistry (2016).

124. Thingholm, T. E. \& Larsen, M. R. The use of titanium dioxide for selective enrichment of phosphorylated peptides. in Methods in Molecular Biology (2016).

125. Larsen, M. R., Thingholm, T. E., Jensen, O. N., Roepstorff, P. \& Jørgensen, T. J. D. Highly selective enrichment of phosphorylated peptides from peptide mixtures using titanium dioxide microcolumns. Mol Cell Proteomics (2005).

126. Li, Y. et al. Fe3O4@ Al2O3 magnetic core-shell microspheres for rapid and highly specific capture of phosphopeptides with mass spectrometry analysis. J Chromatogr A (2007). 
127. Han, L. et al. Mesoporous Fe2O3 microspheres: Rapid and effective enrichment of phosphopeptides for MALDI-TOF MS analysis. J Colloid Interface Sci (2008).

128. Kweon, H. K. \& Håkansson, K. Selective zirconium dioxide-based enrichment of phosphorylated peptides for mass spectrometric analysis. Anal Chem (2006).

129. Ficarro, S. B. et al. Phosphoproteome analysis by mass spectrometry and its application to Saccharomyces cerevisiae. Nat Biotechnol (2002).

130. Thingholm, T. E., Jensen, O. N. \& Larsen, M. R. Enrichment and separation of mono- and multiply phosphorylated peptides using sequential elution from IMAC prior to mass spectrometric analysis. Methods in molecular biology (Clifton, N.J.) (2009).

131. Thingholm, T. E. \& Larsen, M. R. Sequential elution from IMAC (SIMAC): An efficient method for enrichment and separation of mono-and multi-phosphorylated peptides. in Methods in Molecular Biology (2016).

132. Grønborg, M. et al. A mass spectrometry-based proteomic approach for identification of serine/threonine-phosphorylated proteins by enrichment with phospho-specific antibodies: identification of a novel protein, Frigg, as a protein kinase A substrate. Mol Cell Proteomics (2002).

133. Kaufmann, H., Bailey, J. E. \& Fussenegger, M. Use of antibodies for detection of phosphorylated proteins separated by two-dimensional gel electrophoresis. Proteomics (2001).

134. Pinkse, M. W. H. et al. Highly robust, automated, and sensitive online TiO2-based phosphoproteomics applied to study endogenous phosphorylation in Drosophila melanogaster. J Proteome Res (2008).

135. Yao, Y. et al. SH2 Superbinder Modified Monolithic Capillary Column for the Sensitive Analysis of Protein Tyrosine Phosphorylation. J Proteome Res (2018).

136. Von Stechow, L., Francavilla, C. \& Olsen, J. V. Recent findings and technological advances in phosphoproteomics for cells and tissues. Expert Review of Proteomics (2015).

137. Nühse, T. S., Stensballe, A., Jensen, O. N. \& Peck, S. C. Large-scale analysis of in vivo phosphorylated membrane proteins by immobilized metal ion affinity chromatography and mass spectrometry. Mol Cell Proteomics (2003).

138. Emgenbroich, M. et al. A phosphotyrosine-imprinted polymer receptor for the recognition of tyrosine phosphorylated peptides. Chem - A Eur J (2008).

139. Haginaka, J., Tabo, H. \& Matsunaga, H. Preparation of molecularly imprinted polymers for organophosphates and their application to the recognition of organophosphorus compounds and phosphopeptides. Anal Chim Acta (2012).

140. Helling, S. et al. Ultratrace enrichment of tyrosine phosphorylated peptides on an imprinted polymer. Anal Chem (2011).

141. Chen, J. et al. Validation of molecularly imprinted polymers for side chain selective phosphopeptide enrichment. J Chromatogr A (2016).

142. Bllaci, L. et al. Phosphotyrosine Biased Enrichment of Tryptic Peptides from Cancer Cells by Combining pY-MIP and TiO2 Affinity Resins. Anal Chem (2017).

143. Sellergren, B. et al. Selective enrichment of histidine phosphorylated peptides using molecularly imprinted polymers. Anal Chem (2021).

144. Li, Q. et al. One-pot synthesis of phenylphosphonic acid imprinted polymers for tyrosine phosphopeptides recognition in aqueous phase. Anal Chim Acta (2013).

145. Griffiths, J. A brief history of mass spectrometry. Analytical Chemistry (2008). 
146. Karas, M. \& Hillenkamp, F. Laser Desorption Ionization of Proteins with Molecular Masses Exceeding 10000 Daltons. Analytical Chemistry (1988).

147. Zaluzec, E. J., Gage, D. A. \& Watson, J. T. Matrix-Assisted Laser Desorption Ionization Mass Spectrometry: Applications in Peptide and Protein Characterization. Protein Expression and Purification (1995).

148. Ho, C. S. et al. Electrospray ionisation mass spectrometry: principles and clinical applications. Clin Biochem Rev (2003).

149. Savaryn, J. P., Toby, T. K. \& Kelleher, N. L. A researcher's guide to mass spectrometry-based proteomics. Proteomics (2016).

150. Kjellstrtöm, S. \& Jensen, O. N. Phosphoric acid as a matrix additive for MALDI MS analysis of phosphopeptides and phosphoproteins. Anal Chem (2004).

151. Senko, M. W. et al. Novel parallelized quadrupole/linear ion trap/orbitrap tribrid mass spectrometer improving proteome coverage and peptide identification rates. Anal Chem (2013).

152. Medhe, S. Chemical and Biomolecular Engineering Mass Spectrometry: Detectors Review. Mass Spectrom Detect Rev Artic Annu Rev Chem Biomol Eng (2018).

153. Larsen, M. R., Trelle, M. B., Thingholm, T. E. \& Jensen, O. N. Analysis of posttranslational modifications of proteins by tandem mass spectrometry. BioTechniques (2006).

154. Coon, J. J., Syka, J. E. P., Shabanowitz, J. \& Hunt, D. F. Tandem mass spectrometry for peptide and protein sequence analysis. BioTechniques (2005).

155. Haddon, W. F. \& McLafferty, F. W. Metastable Ion Characteristics. VII. Collision-Induced Metastables. Journal of the American Chemical Society (1968).

156. Olsen, J. V. et al. Higher-energy C-trap dissociation for peptide modification analysis. Nat Methods (2007).

157. Zubarev, R. A. et al. Electron capture dissociation for structural characterization of multiply charged protein cations. Anal Chem (2000).

158. Syka, J. E. P., Coon, J. J., Schroeder, M. J., Shabanowitz, J. \& Hunt, D. F. Peptide and protein sequence analysis by electron transfer dissociation mass spectrometry. Proc Natl Acad Sci U S A (2004).

159. Rachkov, A. \& Minoura, N. Towards molecularly imprinted polymers selective to peptides and proteins. The epitope approach. Biochim Biophys Acta - Protein Struct Mol Enzymol (2001).

160. Barišić, D., Cindro, N., Vidović, N., Bregović, N. \& Tomišić, V. Protonation and anion-binding properties of aromatic sulfonylurea derivatives. RSC Adv (2021).

161. Hall, A. J. et al. Urea host monomers for stoichiometric molecular imprinting of oxyanions. J Org Chem (2005).

162. Wulff, G., Gross, T. \& Schönfeld, R. Enzyme Models Based on Molecularly Imprinted Polymers with Strong Esterase Activity. Angew Chemie (International Ed English) (1997).

163. Steed, J. W. \& Atwood, J. L. Supramolecular chemistry 2nd edition. Wiley (2009).

164. Caltagirone, C. \& Gale, P. A. Anion receptor chemistry: highlights from 2007. Chem Soc Rev (2009).

165. Blažek Bregović, V., Basarić, N. \& Mlinarić-Majerski, K. Anion binding with urea and thiourea derivatives. Coordination Chemistry Reviews (2015).

166. Fan, E., Van Arman, S. A., Kincaid, S. \& Hamilton, A. D. Molecular Recognition: HydrogenBonding Receptors That Function in Highly Competitive Solvents. J Am Chem Soc (1993). 
167. Amendola, V. et al. Some guidelines for the design of anion receptors. Coordination Chemistry Reviews (2006).

168. Madarász, Á. et al. Thiourea Derivatives as Brønsted Acid Organocatalysts. ACS Catal (2016).

169. Gunnlaugsson, T. et al. Fluorescent Photoinduced Electron Transfer (PET) sensors for anions; From design to potential application. J Fluoresc (2005).

170. Jose, D. A. et al. Role of positional isomers on receptor-anion binding and evidence for resonance energy transfer. Tetrahedron (2007).

171. Wittkopp, A. \& Schreiner, P. R. Metal-free, noncovalent catalysis of Diels - Alder reactions by Neutral hydrogen bond donors in organic solvents and in water. Chem - A Eur J (2003).

172. Lippert, K. M. et al. Hydrogen-bonding thiourea organocatalysts: The privileged 3,5-bis(trifluoromethyl)phenyl group. European J Org Chem (2012).

173. Jakab, G., Tancon, C., Zhang, Z., Lippert, K. M. \& Schreiner, P. R. (Thio)urea organocatalyst equilibrium acidities in DMSO. Org Lett (2012).

174. Shinde, S. et al. Urea-Based Imprinted Polymer Hosts with Switchable Anion Preference. J Am Chem Soc (2020).

175. Thordarson, P. Determining association constants from titration experiments in supramolecular chemistry. Chem Soc Rev (2011). 



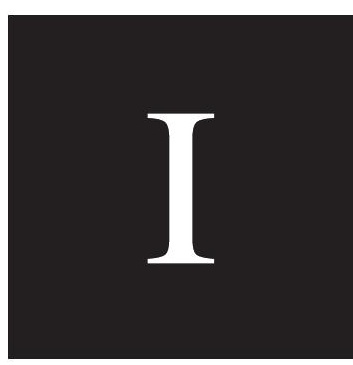





\title{
Urea-Based Imprinted Polymer Hosts with Switchable Anion Preference
}

\author{
Sudhirkumar Shinde, ${ }^{\bigcirc}$ Anil Incel, ${ }^{\bigcirc}$ Mona Mansour, Gustaf D. Olsson, Ian A. Nicholls, Cem Esen, \\ Javier Urraca, and Börje Sellergren*
}

Cite This: J. Am. Chem. Soc. 2020, 142, 11404-11416

\begin{abstract}
ACCESS | 네 Metrics \& More | 回 Article Recommendations | S1 Supporting Information
ABSTRACT: The design of artificial oxyanion receptors with switchable ion preference is a challenging goal in host-guest chemistry. We here report on molecularly imprinted polymers (MIPs) with an external phospho-sulpho switch driven by small molecule modifiers. The polymers were prepared by hydrogen bond-mediated imprinting of the mono- or dianions of phenyl phosphonic acid (PPA), phenyl sulfonic acid (PSA), and benzoic acid (BA) using N-3,5-bis-(trifluoromethyl)-phenyl- $N$-4-vinylphenyl urea (1) as the functional host monomer. The interaction mode between the functional monomer and the monoanions was

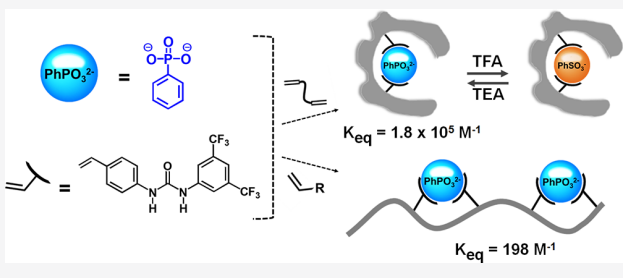
elucidated by ${ }^{1} \mathrm{H}$ NMR titrations and ${ }^{1} \mathrm{H}-{ }^{1} \mathrm{H}$ NMR NOESY supported by molecular dynamic simulation, which confirmed the presence of high-order complexes. PPA imprinted polymers bound PPA with an equilibrium constant $K_{\mathrm{eq}}=1.8 \times 10^{5} \mathrm{M}^{-1}$ in acetonitrile $\left(0.1 \%\right.$ 1,2,2,6,6-pentamethylpiperidine) and inorganic $\mathrm{HPO}_{4}{ }^{2-}$ and $\mathrm{SO}_{4}{ }^{2-}$ with $K_{\mathrm{eq}}=2.9 \times 10^{3} \mathrm{M}^{-1}$ and $4.5 \times 10^{3} \mathrm{M}^{-1}$, respectively, in aqueous buffer. Moreover, the chromatographic retentivity of phosphonate versus sulfonate was shown to be completely switched on this polymer when changing from a basic to an acidic modifier. Mechanistic insights into this system were obtained from kinetic investigations and DSC-, MALDI-TOF-MS-, ${ }^{1} \mathrm{H}$ NMR-studies of linear polymers prepared in the presence of template. The results suggest the formation of template induced 1-1 diad repeats in the polymer main chain shedding unique light on the relative contributions of configurational and conformational imprinting.
\end{abstract}

\section{INTRODUCTION}

The molecular constituents of a living cell are predominantly water-soluble molecules carrying a net negative charge, e.g., cofactors, enzyme substrates, nucleic acids, the lipid double layer, and the glycocalix. ${ }^{1-3}$ As a consequence, evolution has resulted in highly refined receptors capable of recognizing anions in water. Some of the most impressive examples are the proteins designed to selectively bind and/or transport either sulfate or phosphate ions. These two pyramidal anions are nearly isosteric with similar molecular volumes and central atom-oxygen bond lengths, whereas they differ with respect to Lewis basicity and hydrophilicity (Table 1).

Discrimination between phosphate and sulfate and other isosteric oxyanions (e.g., arsenate) occurs in the sulfate and phosphate binding proteins predominantly through multiple complementary hydrogen bonding ( $\mathrm{H}$-bond) interactions involving main chain amides (nests) in a water-poor microenvironment with minor involvement of charge complementary residues. ${ }^{1,4,5}$ Notably, no charged residue is involved in the sulfate binding protein binding site which binds sulfate specifically with an equilibrium binding constant $K_{\mathrm{eq}}=10^{6} \mathrm{M}^{-1}$ in water $(5.0 \leq \mathrm{pH} \leq 8.0),{ }^{5}$ an impressive feat given the strong hydration of this anion. As a consequence, this overturns the Hofmeister series of the salting out tendency for anions which otherwise increases in the order; $\mathrm{CH}_{3} \mathrm{COO}^{-}<\mathrm{HPO}_{4}{ }^{2-}<$ $\mathrm{SO}_{4}^{2-}$.

Inspired by this remarkable performance, much effort has been devoted to the development of neutral sulfate/phosphate binding hosts. ${ }^{4,5}$ In contrast to the more common approach based on charged receptors, neutral hosts can achieve higher selectivity but are challenging to construct since H-bonding is considerably weaker in polar solvents. Nevertheless, both macrocyclic e.g. pyrrole, saphyrine, ${ }^{6}$ peptide ${ }^{7}$ and acyclic podand hosts e.g. ureas, ${ }^{8-11}$ squaramides $^{12-14}$ have been reported showing high sulfate affinity. However, these hosts rarely overturn the Hofmeister solvation governed series of anion affinity especially with respect to the relative preference for phosphate/sulfate. ${ }^{6}$ Moreover, these anion receptors are seldomly capable of switching the anion preference or of binding and releasing the guest upon external stimuli. ${ }^{13}$

Received: January 23, 2020

Published: May 19, 2020 
Table 1. Physical Properties of Sulfate and Phosphate Anions

$\begin{array}{cccccc}\text { anion } & \mathrm{pK}_{\mathrm{a}} 1^{a} & \mathrm{pK}_{\mathrm{a}} 2^{a} & \mathrm{pK}_{\mathrm{a}} 3^{a} & \text { molecular volume }{ }^{b}\left(\AA^{3}\right) & \text { anion hydration energy }{ }^{b}\left(\mathrm{~kJ} / \mathrm{mol}^{2}\right) \\ \mathrm{PO}_{4}{ }^{3-} & 2.1 & 7.2 & 10.9 & 56 & -498 \\ \mathrm{SO}_{4}{ }^{2-} & -3 & 1.9 & & 51 & -1130 \\ { }^{a} \mathrm{pK} K_{\mathrm{a}} \text { of conjugate acid. }{ }^{b} \text { Calculated values assuming } \mathrm{pH} & =6 \text { and an average charge of }-2 \text { for sulfate and }-1.4 \text { for phosphate. }^{3}\end{array}$

This highlights a need of neutral hosts with tunable microenvironment capable of alternating phosphate/sulfate recognition. This can in principle be achieved by embedding designed low molecular weight hosts in a polymer scaffold imparting a lower dielectric microenvironment. ${ }^{1}$ Alternatively, a macromolecular host can be designed from simpler building blocks by molecular imprinting. ${ }^{15-20}$ Monomers are here chosen or designed to complement functional groups of a template molecule. Polymerizing the monomer-template complexes into a cross-linked polymer matrix followed by removal of the template, leaves behind sites capable of recognizing the template. This approach benefits from spontaneous self-assembly guiding the binding groups to their positions in the receptor site. Thus, the structure of the final binding site is a priori unknown.

Notable examples of MIPs for anion recognition in water have been reported using both charged, e.g., imidazolium, ${ }^{21-23}$ amidinium, ${ }^{24-27}$ and neutral host monomers, e.g., urea, ${ }^{28-31}$ thiourea, ${ }^{32}$ squaramide, ${ }^{33}$ and others. ${ }^{34}$ Some time ago we introduced urea based host monomers acting as potent $\mathrm{H}$ bond donors for stoichiometric imprinting of oxyanions or other H-bond acceptors. ${ }^{35}$ Receptors for $\beta$-lactam antibiotics, ${ }^{36}$ phospho-peptides, ${ }^{28}$ peptide biomarkers, ${ }^{37}$ phospholipids, ${ }^{22}$ sugar acids, ${ }^{31}$ and sulfated peptides ${ }^{29}$ were demonstrated.

To gain insight into the nature of these imprinted receptors at a molecular level we here report an in-depth investigation of MIPs for simple oxyanions (Scheme 1).

Scheme 1. Plausible Structural Formulas of H-Bonded Complex between Oxyanions and 1,3-Diaryl Urea Monomer 1 (left) and Structures of Oxyacids Used in the Study (Right) ${ }^{a}$

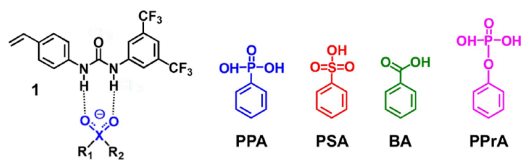

${ }^{a} \mathrm{PPA}=$ phenylphosphonic acid; PSA $=$ phenylsulfonic acid; $\mathrm{BA}=$ benzoic acid; and PPrA = phenylphosphoric acid.

With this we aimed to answer the following questions: (1) What level of oxyanion selectivity and affinity can be achieved with MIPs in organic and aqueous media? (2) Can MIPs recognize inorganic anions in water? (3) Can the Hofmeister preference be overturned? (4) Can we switch oxyanion preference using the same MIP? (5) What are the mechanisms underlying the imprinting process?

On the basis of combined physical characterization, modeling, spectroscopic studies of prepolymerization complexes, and studies of the polymerization kinetics we here offer answers to the aforementioned questions.

\section{RESULTS AND DISCUSSION}

Monomer/Template Complex Formation. 1,3-Disubstituted ureas have long been exploited as neutral hosts $(\mathrm{H})$ for complexing oxyanion guests $(\mathrm{G}){ }^{38,39}$ They provide a 2 -fold $\mathrm{H}$ bond donor which with the acceptor (e.g. carboxylate, phosphate, sulfate) results in a cyclic H-bonded complex. Also, several structural and compositional parameters can be readily tuned to enhance $\mathrm{HG}$ association strengths (Scheme 2).

Scheme 2. Structural and Compositional Parameters for Tuning Host-Guest Association Strength ${ }^{a}$

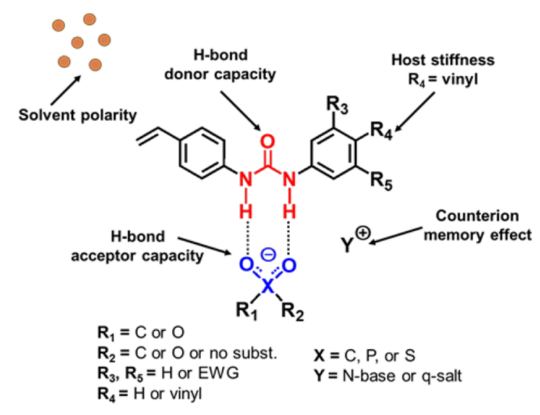

${ }^{a} \mathrm{EWG}=$ electron withdrawing group.

The affinity for the guest increases with the acidity of the urea protons (donor capacity, $\mathrm{R}_{3}, \mathrm{R}_{4}, \mathrm{R}_{5}=$ electron withdrawing groups (EWGs)) and the basicity of the oxyanion (acceptor capacity), but this can be offset by the ability of the host to self-associate and host deprotonation. ${ }^{40}$ The latter leads to nondirectional electrostatic interactions and is promoted by increased solvent polarity. ${ }^{41}$ This is an undesired effect since it undermines the structural integrity of the hydrogen bond connected HG complex-hence a compromise between host acidity and guest basicity has to be found that maximizes the complex strength. In this context, no deprotonation has so far been observed for the host monomer, 1, used in the current study (Scheme 1). The nature of the countercation also influences the HG complex stability. Complexation can be easily induced by proton transfer to amine bases but this causes undesirable competition between the urea host and the protonated amine for binding the guest. Stronger complexation is therefore induced by the use of bulky tertiary amines (e.g., pentamethylpiperidine, PMP) or quaternary ammonium ions like tetrabutylammonium (TBA), which have been shown to produce pronounced counterion memory effects. ${ }^{28,42}$ Another factor is the level of host preorganization, which impacts on the size of the entropic penalty upon guest complexation due to the need to freeze internal rotors. For monourea host monomers this can in principle be enhanced by introducing two polymerizable groups (cross-linkers) in the host thereby 
increasing host stiffness $\left(\mathrm{R}_{4}=\right.$ vinyl in Scheme 2). ${ }^{41}$ For ternary HG complexes formed from phosphate dianions and urea hosts (2:1 complex in Scheme 3), host preorganization has been enhanced by introducing the cleft-like host monomer 2 (1:1 complex in Scheme 3)..$^{28}$

Scheme 3. Anticipated Ternary or Binary MonomerTemplate Complexes Formed Using Mono- (Left) Or Di(Right) Functional Urea Host Monomers

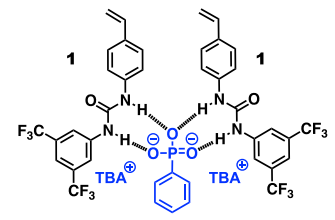

2:1 complex

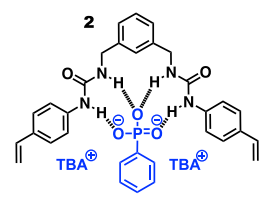

1:1 complex
Considering oxyanion-oxourea complexation, the stability has been shown to increase in the order of increasing acceptor basicity, i.e., $\mathrm{PhSO}_{3}{ }^{-}<\mathrm{PhOP}(\mathrm{OH}) \mathrm{O}_{2}{ }^{-}<\mathrm{PhP}(\mathrm{OH}) \mathrm{O}_{2}{ }^{-} \leq$ $\mathrm{PhCOO}^{-}<\mathrm{PhPO}_{3}{ }^{2}-\leq \mathrm{PhOPO}_{3}{ }^{2-} \cdot{ }^{43}$ To verify this trend we decided to investigate 1 with respect to its complex stability with model anions in the form of TBA-salts of PPA, PPrA, BA, and PSA. Interaction strength and complex stoichiometries were investigated through ${ }^{1} \mathrm{H}$ NMR titration or isothermal titration calorimetry (ITC) (see the Supporting Information (SI)). Titrating 1 with PPA.TBA in DMSO- $\mathrm{d}_{6}$ and PPA.2PMP in THF- $\mathrm{d}_{8}$ resulted in strong downfield shifts of urea protons $\mathrm{Ha}$ and $\mathrm{Hb}$ and more moderate shifts of the aromatic protons Hc-f (Figure S1). The moderate downfield shift of $\mathrm{He}$ and upfield shift of Hf are in agreement with previous NMR titration study of 1,3-bis(4-nitrophenyl)urea with acetate. ${ }^{44}{ }^{1} \mathrm{H}$ NMR studies using Jobs method of continuous variation confirmed a preferred 1:1 stoichiometry. Complex stability constants were calculated using the induced downfield shifts of the urea protons of $\mathbf{1}$ (Figure S1). With respect to monoanions, $\mathbf{1}$ formed more stable complexes with benzoate anion BA $\left(K_{\mathrm{eq}}=8820 \mathrm{M}^{-1}\right)$ followed by PPA $\left(K_{\mathrm{eq}}=7005\right.$ $\left.\mathrm{M}^{-1}\right)$ and $\operatorname{PPrA}\left(K_{\mathrm{eq}}=676 \mathrm{M}^{-1}\right)$, whereas the complex with the sulfonate PSA was too weak to be detected (Table S1). This agrees with the trend determined by Kelly et al. ${ }^{43}$ Comparing with monoanions, the dianions of PPA and PPrA interact more strongly with $1 .{ }^{28}$ The relative affinity of the two dianions is reflected in the downfield shifts of $\mathrm{Ha}$ and $\mathrm{Hb}$ (Figures 1 and 2) where PPrA (Figure 1c) display larger shifts than PPA (Figure 1b), again in line with the literature results.

To gain more insight we performed molecular dynamics (MD) simulations of the prepolymerization mixtures. MD simulations offer unique insights into the nature of the interactions between polymerization mixture components providing diagnostic/prognostic correlation to polymer performance. ${ }^{46,47}$ Compositions of simulated and evaluated systems and chemical structures are presented in Table 2 and Figure S15.

Full system all-atom simulations were performed using the same stoichiometries employed for polymer synthesis. The results overall confirmed the presence of stable interactions between the neutral, mono-, and di-valent anions of PPA and $\mathbf{1}$ (Tables 3 and S8-S10 and Figure S15) with the dianion of PPA (PP2) (PPrA not investigated using MD) displaying a

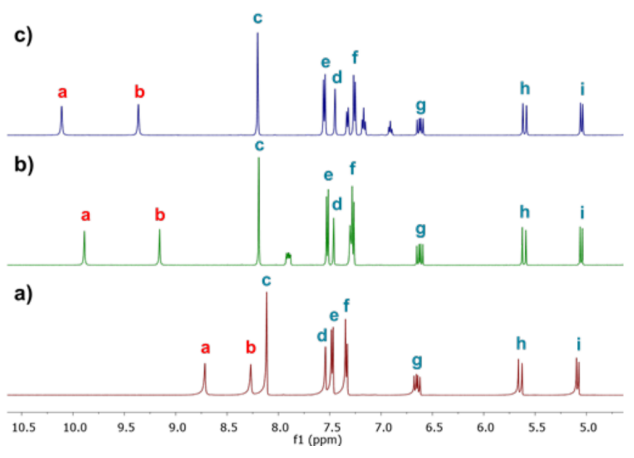

Figure 1. ${ }^{1} \mathrm{H}$ NMR spectra of (a) 1 and (b) 2:1 ratio of 1:PPA.2PMP and (c) 2:1 ratio of 1:PPrA.2PMP in THF- $\mathrm{d}_{8}$. [1] $=30 \mathrm{mM}$.<smiles>CN(C(=O)N(C)c1ccc(C(F)(F)F)cc1F)c1ccc(C(F)(F)F)cc1</smiles>

$\Delta \delta$ PPA
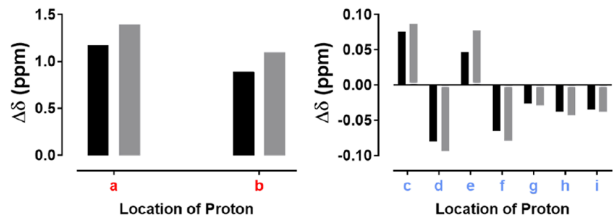

Figure 2. ${ }^{1} \mathrm{H}$ NMR chemical shift changes of urea host monomer 1 complexation with PPA.2PMP or PPrA.2PMP. $\Delta \delta=\delta$ (in the presence of anion) $-\delta$ (in the absence of anions), induced by addition of $1 / 2$ equiv of different anions to receptor 1 .

higher degree of H-bonding than the monoanion (PP1) or neutral PPA template.

However, the monoanionic PSA template (PS1) performs as well, even slightly better than PP2 considering complex formation with 1 (Tables S8-S10). A rank order of affinity for 1 was observed where anionic neutral PSA $<$ neutral PPA $<$ anionic PP1 < dianionic PP2 < PS1 (Tables 3 and S8-S10). This obviously disagrees with the NMR results where no interactions between PS1 and 1 could be detected. The systems differ though with respect to solvent polarity, the base, presence of cross-linker, and the concentration of all species which we believe can account for these contrasting results (vide infra). ${ }^{45-48}$

To gain further insight into the nature of the urea complexes between 1 and the two anions we performed 2D-NOESY NMR experiments and molecular modeling as reported by others. ${ }^{11}$ As shown in Figure 3a the free 1 shows strong crosspeaks with the residual water signal at ca. $2.9 \mathrm{ppm}$ indicating water protons close in space to the urea protons. In addition, weaker cross-peaks are observed between the two-urea protons $\mathrm{Ha}$ and $\mathrm{Hb}$ resulting from the $Z, Z$-urea conformer with the two protons juxtaposed (Figure $3 \mathrm{e}$ ). The addition of half an equivalent of PPA.2PMP (Figure 3b) and PPrA.2PMP (Figure 
Table 2. Composition of Systems Simulated and Evaluated ${ }^{a}$

\begin{tabular}{lllllllll}
\multicolumn{1}{c}{$c$} & P1N & P1C & P2N & P2C & P3N & P3C & P4N & P4C \\
$T^{b}$ & PPA & PP1 & PPA & PP2 & PSA & PS1 & PSA \\
PMP $^{b}$ & 20 & & 40 & & 40 & 20 & 20 & 40 \\
PMPH $^{+b}$ & & 20 & 20 & 40 & 40 & 20 & 20 & 40 \\
1 & 20 & 800 & 800 & 800 & 800 & 800 & 800 \\
EGDMA $^{b}$ & 800 & 2760 & 2760 & 2760 & 2760 & 2760 & 800 \\
THF & 2760 & 2760 &
\end{tabular}

${ }^{a}$ Numbers of virtual molecular models of each molecule included in performed simulations of systems. See Figure S15 for molecular representations and defined abbreviations. ${ }^{b} T=$ template $(20$ equiv $)$, PP $1=$ PPA monoanion, PP $2=$ PPA dianion, PS $1=$ PSA monoanion, PMP $=$ 1,2,2,6,6-pentamethylpiperidine, EGDMA = ethylenglycoldimethacrylate. ${ }^{c} \mathrm{PXN} / \mathrm{C}$, Polymer system X, neutral (N) or charged (C).

Table 3. H-Bond Analysis Results ${ }^{a}$

\begin{tabular}{|c|c|c|c|c|c|c|c|}
\hline & & $T^{b}$ & PMP & $\mathrm{PMPH}^{+}$ & 1 & EGDMA & THF \\
\hline \multirow[t]{3}{*}{ P1C } & PP1 & 0.00 & & 0.00 & 0.11 & 0.00 & 0.00 \\
\hline & $\mathrm{PMPH}^{+}$ & 64.54 & & 0.00 & 0.00 & 0.00 & 0.01 \\
\hline & 1 & 24.26 & & 0.00 & 0.11 & 18.27 & 9.50 \\
\hline \multirow[t]{2}{*}{ P1N } & PPA & 77.27 & 0.00 & & 0.04 & 46.69 & 18.41 \\
\hline & 1 & 13.42 & 0.00 & & 0.10 & 25.93 & 12.96 \\
\hline \multirow[t]{2}{*}{$\mathrm{P} 2 \mathrm{C}$} & $\mathrm{PMPH}^{+}$ & 23.42 & & 0.00 & 0.00 & 0.00 & 0.00 \\
\hline & 1 & 74.37 & & 0.00 & 0.27 & 15.11 & 7.55 \\
\hline \multirow[t]{2}{*}{$\mathrm{P} 2 \mathrm{~N}$} & PPA & 38.96 & 0.00 & & 0.09 & 77.59 & 14.66 \\
\hline & 1 & 33.57 & 0.00 & & 0.50 & 48.62 & 24.68 \\
\hline \multirow[t]{2}{*}{ P3C } & $\mathrm{PMPH}^{+}$ & 75.69 & & 0.00 & 0.00 & 0.00 & 0.13 \\
\hline & 1 & 47.39 & & 0.00 & 0.05 & 11.84 & 6.02 \\
\hline \multirow[t]{2}{*}{ P3N } & PSA & 0.08 & 0.00 & & 0.02 & 2.80 & 2.00 \\
\hline & 1 & 1.06 & 0.00 & & 0.36 & 29.18 & 14.78 \\
\hline \multirow[t]{2}{*}{ P4C } & $\mathrm{PMPH}^{+}$ & 80.58 & 0.00 & 0.00 & 0.00 & 0.00 & 0.01 \\
\hline & 1 & 85.22 & 0.00 & 0.00 & 0.19 & 16.85 & 7.15 \\
\hline \multirow[t]{2}{*}{ P4N } & PSA & 0.08 & 0.00 & & 0.05 & 2.76 & 2.00 \\
\hline & 1 & 1.50 & 0.00 & & 0.39 & 30.18 & 15.69 \\
\hline
\end{tabular}

${ }^{a}$ Summarized average interactions, as percent of total simulation time, between all atom pairs of indicated molecular species. See Figure S15 for molecular representations and defined abbreviations. All analyses involving templates were averaged against the number of templates ( 20 in each system). All interactions including the urea monomer 1 but not the template was averaged against the number of urea monomer molecules in each system (see Table 2). Cross-linker interactions with solvent and bases were averaged against the number of cross-linkers ( 800 in all systems) and, finally, base self-interaction and solvent interaction were averaged against the number of base molecules in each system (20 in P1, P3 N/C systems and P4C, 40 in P2 N/C and P4N systems). ${ }^{b}$ Interactions with the template in the analyzed system.

3c) to 1 gave rise to a stronger NOE between these protons and an interesting crosspeak between the more acidic $\mathrm{Ha}$ proton and the exchangeable $\mathrm{PMPH}^{+}$proton, visible as a broad signal at $3.5 \mathrm{ppm}$ (Figure S1e).

The former reflects stabilization of the Z,Z-conformer, whereas the latter a close contact between 1 and PMP, this providing an indirect support for the existence of the anticipated ternary complex between 1, PMP and the dianions (Scheme 4) This is in agreement with previous reports on urea- and squaramide-based sulfate receptors. ${ }^{12,49}$ Results from MD simulations also support the argument for higher order complexes observed in cameo images from the production run (Figures S16 and S17) and in the radial distribution function (RDF) analyses (Table 4).

As expected, the degree of complexation increases with the template charge, e.g., P2C ( $\mathrm{T}=$ PPA dianion $)$ display clusters involving any part of 6 molecules of 1 within $5 \AA$ from a template molecule, whereas only half of this number is seen in P1C. Generally, $\mathrm{PMPH}^{+}$was found in the simulations to be in close proximity to the templates and engaged in extensive $\mathrm{H}$ bonding with charged templates (Table 3 ). These results support the observed solubilization of the template PPA.2PMP in the presence of $\mathbf{1}$ in THF (Scheme 4). Whereas the ammonium phosphonate salt was poorly soluble in this solvent, the solution became completely clear after addition of 2 equiv of 1 . This provides unequivocal visual proof for the presence of prepolymerization complexes between 1 and PPA. 2PMP.

Polymer preparation and characterization. Imprinted and nonimprinted polymers were thereafter prepared and characterized (see SI and Tables S2-S4) using the urea host monomer 1 in a 2:1 and 1:1 stoichiometric ratio to the templates: PPA, PSA, or BA in their monoanionic or dianionic forms (Scheme 4). Nonimprinted polymers $\left(P_{N}\right)$ were prepared identically to the imprinted polymers though in the absence of template. Figure S2 shows the $25-36 \mu \mathrm{m}$ particle fraction obtained after crushing and sieving of the polymer monoliths and template removal by solvent extraction. These were subjected to physical characterization as follows: C, H, and $\mathrm{N}$ elemental analysis of imprinted and nonimprinted polymers after removal of template matched calculated values after correction for water uptake. This is reflected in the agreement between calculated and found $\mathrm{N} / \mathrm{C}$ ratios (Table S3). Thermogravimetric analysis (TGA) of the polymers produced weight loss curves typical for highly cross-linked networks with onset above $200{ }^{\circ} \mathrm{C}$ (Figure S3) and complete weight loss obtained at $450{ }^{\circ} \mathrm{C}$. Meanwhile, the transmission FTIR spectra (Figure S4) showed all characteristic bands with 

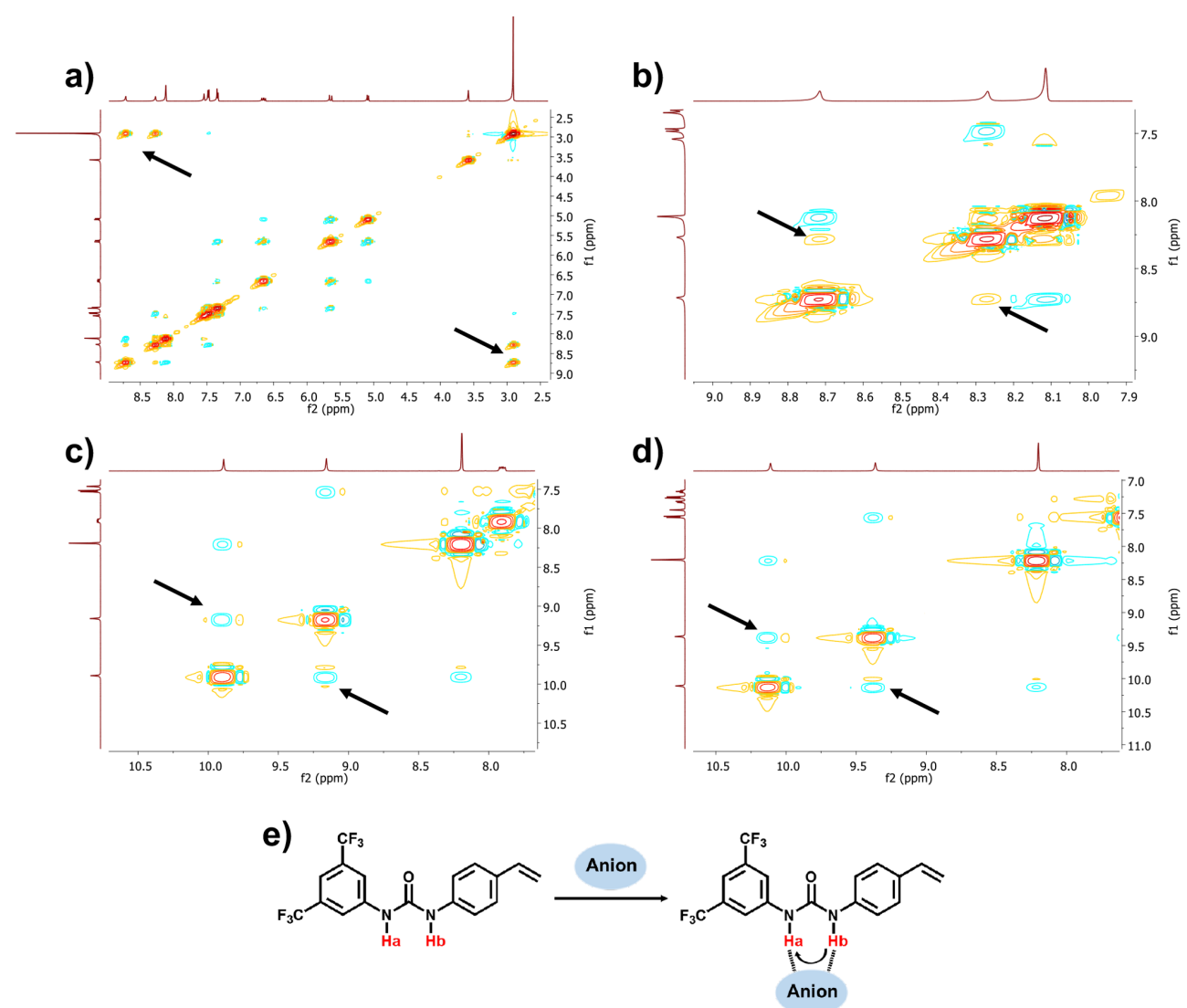

Figure 3. ${ }^{1} \mathrm{H}-{ }^{1} \mathrm{H}$ NMR NOESY spectra of 1: (a) and (b) (close-up), (c) 2:1 ratio of 1:PPA-2PMP and (d) 2:1 ratio of 1:PPrA-2PMP in THF- $d_{8}$ and (e) anion induced conformational stabilization detected by an increase ${ }^{1} \mathrm{H}-{ }^{1} \mathrm{H}$ NMR NOESY between $\mathrm{Ha}$ and $\mathrm{Hb}$. [1] $=30 \mathrm{mM}$. The arrows indicate the urea-water $(\mathrm{a})$ and urea $-\mathrm{Ha}, \mathrm{Hb}$ cross peaks $(\mathrm{b}-\mathrm{d})$.

Scheme 4. Solubilization of the Bis-PMP-Salt of PPA by the Addition of 2 Equiv of Urea Host Monomer 1 in THF and Procedure for Imprinting

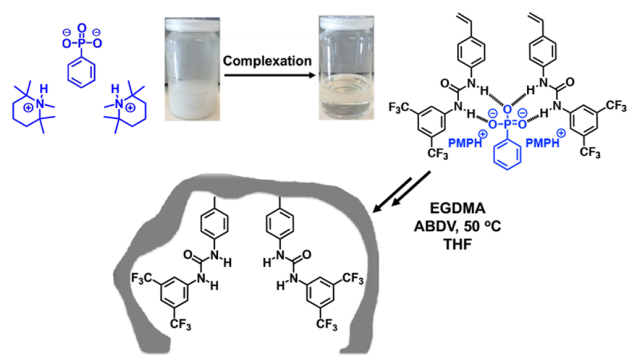

no apparent difference between imprinted and nonimprinted polymers all in all, indicating a stoichiometric monomer
Table 4. Numbers of Molecules within a 5.05 Å Cutoff from Each Template $^{a}$

$\begin{array}{lcccccc} & \text { EGDMA } & \text { PMPH }^{+} & \text {PMP } & \text { T } & \text { THF } & 1 \\ \text { T-P1C } & 8 & 13 & & 0 & 15 & 3 \\ \text { T-P1N } & 12 & & 0 & 5 & 17 & 1 \\ \text { T-P2C } & 5 & 33 & & 0 & 7 & 6 \\ \text { T-P2N } & 13 & & 0 & 2 & 17 & 3 \\ \text { T-P3C } & 8 & 14 & & 1 & 14 & 4 \\ \text { T-P3N } & 12 & & 0 & 0 & 21 & 1 \\ \text { T-P4C } & 8 & 11 & 0 & 0 & 14 & 6 \\ \text { T-P4N } & 12 & & 1 & 0 & 20 & 1\end{array}$

${ }^{a}$ Values presented are obtained through integration of RDFs, assuming the template as the solute molecule with an average number of molecules within the cutoff for an average template.

incorporation and successful template removal from the imprinted polymers. 
This contrasted with the results from nitrogen sorption analysis and swelling tests, both reflecting the porous and structural properties of the polymers (Figure S5, Table S4). All polymers exhibited mesoporous morphology with surface areas exceeding $200 \mathrm{~m}^{2} \mathrm{~g}^{-1}$ and average pore diameters of roughly 4 $\mathrm{nm}$. However, in agreement with our previous report, ${ }^{28}$ the imprinted polymers showed a lower surface area and a higher swelling factor than the nonimprinted polymers. We tentatively attributed this template-induced difference in morphology to two effects of the divalent anion, (1) its action as a physical cross-linking agent and (2) its action as a good solvent for the growing chains leading to delayed phase separation and a more gel-like morphology. Below we will invoke a third alternative explanation to the observed differences based on the dianions ability to coordinate two monomer units of $\mathbf{1}$ in a geometry favoring cyclopolymerization (vide infra).

Template Binding Studies. To evaluate the anion recognition properties of the materials we first assessed their ability to rebind their corresponding templates under static conditions. The polymers were incubated in acetonitrile in presence or absence of base (PMP) with the anion templates (Figure 4) followed by quantification of unbound template by HPLC.

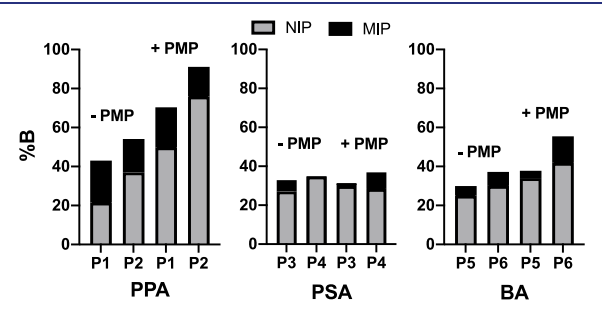

Figure 4. Absorbed amount in percent of PPA (a), PSA (b), and BA (c) by anion imprinted and nonimprinted polymers in the absence and presence of $0.1 \%$ PMP modifier.

Figure 4 shows the degree of template binding to the polymers in presence or absence of PMP. MIPs generally bound more of the templates than nonimprinted polymers (NIPs) with the largest uptake shown by the polymers prepared from a 2:1 monomer/template ratio and with a significant increase observed upon PMP initiated deprotonation. These results are all in line with the anticipated binding model and both observations correlate with the MD simulation results, whereby increasing the amount of functional monomer in the prepolymerization mixtures increases the extent of template H-bonding (Table 3). Furthermore, template complexation with PPA increases with the degree of deprotonation (neutral < monoanion < dianion). Increasing the amount of PMP will increase the degree of template deprotonation, which increases the degree of interaction with 1. Ranking the polymers in order of decreasing uptake showed that only PPA, and to a lesser extent BA, led to polymers exhibiting these effects, whereas the PSA imprinted polymers lacked significant affinity for the template. This agrees with the weak basicity of the corresponding anions and weak interactions observed for this anion in aprotic solvents. The poor performance of the BA-MIPs P5 and P6 however, disagrees with the basicity trend. To understand the reason for this, the differencies in anion size and functionality should be considered. PPA monoanion is larger and features an additional $\mathrm{H}$-bond donor in $\mathrm{PhP}(\mathrm{OH}) \mathrm{O}_{2}{ }^{-}$which can interact with the polymer. This is lacking in $\mathrm{PhCO}_{2}^{-}$. In view of the observed trends, we chose to characterize the PPA imprinted polymers in more detail.

Adsorption Isotherms and Binding Parameters. The binding-energy distribution of the polymers was obtained from single-component adsorption isotherms determined by a batch equilibrium binding experiment. ${ }^{50}$ The binding curves of PPA on $\mathrm{P} 1 / \mathrm{P}_{\mathrm{N}} 1$ and $\mathrm{P} 2 / \mathrm{P}_{\mathrm{N}} 2$ in acetonitrile buffered with PMP are seen in Figure $5 \mathrm{a}$ and $5 \mathrm{~b}$. Comparing different binding

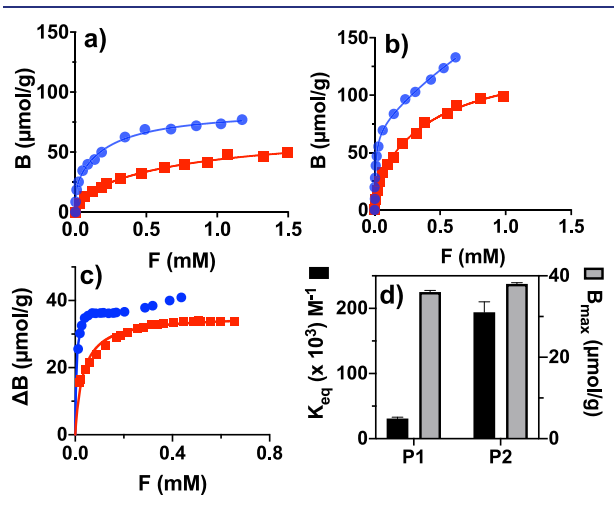

Figure 5. Equilibrium binding isotherms of PPA adsorbed to P1 (blue circles) and $\mathrm{P}_{\mathrm{N}} 1$ (red squares) (a) and $\mathrm{P} 2$ (blue circles) and $\mathrm{P}_{\mathrm{N}} 2$ (red squares) (b) in acetonitrile (0.1\% PMP). In (c) the binding to P1 (red squares) and P2 (blue circles) corrected for binding to the corresponding nonimprinted polymers is shown. The isotherms were fitted to a Langmuir binary-site (a, b) or monosite (c) models resulting in the binding parameters listed in Table 5 . In $c$, the data points above $X=0.2$ have been excluded from the fitting of the data for P2. (d) shows the binding parameters obtained from Figure $5 \mathrm{c}$.

equations (Figure S6) showed that the data were best fitted to the Langmuir binary site model. The associated binding parameters are given in Table 5. The curves exhibited saturation behavior with $\mathrm{P} 2 / \mathrm{P}_{\mathrm{N}} 2$ taking up approximately two times more PPA at a given free concentration. This is in line with the nominal capacity of the materials, which is two times higher for $\mathrm{P} 2 / \mathrm{P}_{\mathrm{N}} 2$ than $\mathrm{P} 1 / \mathrm{P}_{\mathrm{N}} 1$ and correlates with results derived from $\mathrm{MD}$ simulations. The NIPs displayed consistently less binding than the MIPs with the corresponding binding curves rapidly diverging in the low concentration interval of $0-0.5 \mathrm{mM}$. This appears clearly in the plots of the differential uptake in Figure $5 \mathrm{c}$ reflecting MIP binding corrected for binding to the NIP (assuming the latter to reflect the nonspecific binding contribution). Both of these curves fitted with a Langmuir monosite model resulted in nearly identical $B_{\max }$ values reflecting a similar imprinting efficiency of ca. $30 \%$ (given identical template concentration in the prepolymerization mixture) and with P2 showing a markedly higher affinity than P1 (Figure 5d). The latter is reflected in the higher equilibrium constant of $\mathrm{P} 2\left(K_{\mathrm{eq}}=1.9 \times\right.$ $\left.10^{5} \mathrm{M}^{-1}\right)$ versus P1 $\left(K_{\mathrm{eq}}=3.1 \times 10^{4} \mathrm{M}^{-1}\right)$.

This difference can be attributed to the state of template complexation prior to polymerization and is supported by the results obtained from MD simulations. Again, both $\mathrm{H}$-bond 
Table 5. Equilibrium Constants $\left(K_{\mathrm{eq}}\right)$ and Binding Capacities $\left(B_{\max }\right)$ of PPA Imprinted Polymers ${ }^{a}$

$\begin{array}{cccccc}\text { polymer } & \text { site class } & K_{\text {eq }}\left(\times 10^{3} \mathrm{M}^{-1}\right) & B_{\max }(\mu \mathrm{mol} / \mathrm{g}) & R^{2} & \mathrm{IE}^{b}(\%) \\ \mathrm{P} 1 & \text { HI } & 265 \pm 107 & 23 \pm 3 & 0.99 & 18 \\ & \text { LO } & 4.4 \pm 0.8 & 63 \pm 2 & 0.99 & 50 \\ \mathrm{P}_{\mathrm{N}} 1 & \text { HI } & 24 \pm 22 & 14 \pm 8 & 0.99 & \\ & \text { LO } & 1.1 \pm 0.6 & 56 \pm 5 & 0.99 & \\ \mathrm{P} 2 & \text { HI } & 110 \pm 52 & 70 \pm 14 & 0.97 & 56 \\ & \text { LO } & 0.4 \pm 1.2 & 326 \pm 804 & 0.97 & 260 \\ \mathrm{P}_{\mathrm{N}} 2 & \text { HI } & 53 \pm 30 & 24 \pm 8 & 0.99 & \\ & \text { LO } & 2 \pm 0.5 & 115 \pm 5 & 0.99 & \\ \mathrm{P}_{\text {corr }}{ }^{c} & & 31 \pm 2 & 36 \pm 0.4 & 0.96 & 29 \\ \mathrm{P}_{\text {corr }}{ }^{c} & & 194 \pm 16 & 38 \pm 0.3 & 0.96 & 31\end{array}$

${ }^{a}$ The binding parameters were obtained by fitting of the binding data in Figure 5 to a Langmuir monosite $\left(\mathrm{P} 1_{\text {corr }}, \mathrm{P} 2_{\text {corr }}\right)$ or binary site binding model. ${ }^{b}$ Imprinting efficiency IE $=100 \times B_{\max } / B_{\max } *$, where $B_{\max }{ }^{*}=125 \mu \mathrm{mol} / \mathrm{g}=$ nominal capacity assuming quantitative template removal and reoccupancy. ${ }^{c}$ Fitting parameters for isotherms corrected for nonspecific binding to nonimprinted polymer (e.g., $\Delta \mathrm{B}$ $\left.=\mathrm{B}_{\mathrm{P} 1}-\mathrm{B}_{\mathrm{PN} 1}\right)$.

analysis results (Table 3) and the RDF-analysis (Table 4) indicate a higher degree of complexation of $\mathbf{1}$ with the template when increasing the amount of monomer.

On the basis of mass balance equations and assuming the solution complexation constants in Table S1, we predict that PPA is present in the form of a 1:1 complex, whereas for P2, ternary complexes are dominating. Turning to the RDF-results (Table 4), the calculated number of molecules within the given cutoff varies with the cutoff and order of the selected solvent/ solute pairs. However, in the neutrally templated PPA system $\left(\mathrm{P}_{\mathrm{N}} 1\right)$ there is one molecule of 1 within $5 \AA$ of any average template. This number increases to three when either increasing the amount of $\mathbf{1}$ or deprotonating the template PPA to monoanionic PPA. This offers support for the suggested complexation model that indicates the formation of ternary complexes. Increasing the amount of base further leads to dianionic PPA for which RDF results (Table 4) show six molecules of $\mathbf{1}$ in close proximity to any template. The results obtained moving from $1: 1$, through $3: 1$ to $6: 1$ stoichiometries of 1 and template complexes, in association with the base driven increase in degree of deprotonation, add support for the formation of ternary complexes.

Assuming that the structures of the prepolymerization complexes are carried into the polymer scaffold, the 1:2 complex will result in a cleft-like receptor featuring four $\mathrm{H}$ bond donors, whereas the 1:1 complex will yield a site with a 2fold H-bond donor. Accounting for the entropic gain, the resulting binding constant can exceed that of the $1: 1$ site by $2-$ 3 orders of magnitude, which is clearly not the case here. Plausible explanations for this disagreement will be discussed below.

Switchable Oxyanion Selectivity. The crushed polymer monoliths of $\mathrm{P} 1 / \mathrm{P}_{\mathrm{N}} 1$ and $\mathrm{P} 2 / \mathrm{P}_{\mathrm{N}} 2$ were sieved and packed in columns for chromatographic characterization of their retentive properties for the model oxoacids (Scheme 1). The acids were injected in an acetonitrile rich mobile phase as such or buffered with either triethylamine (TEA) or trifluoroacetic acid (TFA). Basic conditions will promote deprotonation leaving the oxyanions to interact with the MIP via $\mathrm{H}$ bonding. ${ }^{28}$ The decisive role of this factor became obvious in the context of the MD simulations of the imprinting process.
Hence, increasing the extent of deprotonation of PPA strongly influences template complexation with both 1 and $\mathrm{PMPH}^{+}$.

The MIP exhibited strong affinity for its template (PPA) in this mobile phase (Figures 6 and Figures S7) with ca. $75 \%$ of

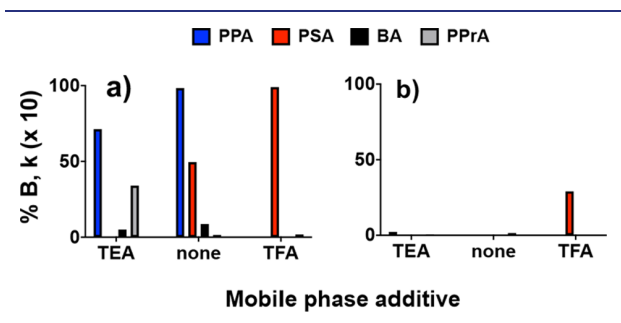

Figure 6. Percent bound (TEA, none) and retention factor $(k \times 10)$ (TFA) for PPA, PSA, BA, and PPrA on columns packed with P2 (a) and $\mathrm{P}_{\mathrm{N}} 2$ (b) using acetonitrile with different modifiers as mobile phases as follows: TEA: Acetonitrile/Water 90:10 (0.1\% TEA); TFA: Acetonitrile/Water 95:5 (0.1\% TFA); and none: 100\% Acetonitrile.

injected analyte remaining stuck on the column. Meanwhile, with the exception of PPrA, none of the other acids were retained whereas $\mathrm{P}_{\mathrm{N}} 2$ exhibited no affinity for any of the analytes. This contrasts with the retention results in the TFAbuffered mobile phase. Here we note a complete switch of anion preference with PSA being the only retained analyte. In absence of modifier both PPA and PSA are retained by P1 and $\mathrm{P} 2$ with no retention observed by nonimprinted $\mathrm{P}_{\mathrm{N}} 1$ and $\mathrm{P}_{\mathrm{N}} 2$.

A detailed look at the ionization states of the acids may offer clues to the origin of the turn on/off effect. Table S5 lists the $\mathrm{p} K_{\mathrm{a}}$ values and anticipated charges in the presence of the two modifiers of the acids in the study. In presence of base, PPA and PPrA carry a net charge of -2 , whereas the monovalent acids are negatively charged -1 . In this state both PPA and PPrA can bind to the P2 site while benefiting from 4 complementary H-bond donors. The PPA dianion is the most strongly retained anion agreeing with the fact that this anion was used as template and hence fits best into the binding sites. The weak retention of the monovalent anion of PSA we ascribe to its weak basicity and its hydrophilicity, whereas planar BA lacks steric complementarity with the PPA templated site. In presence of TFA, however, $\left(\mathrm{p} K_{\mathrm{a}}=0.5\right), \operatorname{PPA}\left(\mathrm{p} K_{\mathrm{a}}=0.9\right)$, and PPA $\left(\mathrm{p} K_{\mathrm{a}}=1.8\right)$ with $\mathrm{p} K_{\mathrm{a}}$ values in vicinity are partially or fully protonated weakening their interactions with the ureas of the stationary phase. PSA $\left(\mathrm{p} K_{\mathrm{a}}=-2.8\right)$ should be fully ionized in water but less so in $95 \%$ acetonitrile in the presence of TFA. As a consequence, PSA is less hydrated under these conditions and can more easily partition into the stationary phase. With only one of the three oxygen acceptors blocked by protonation PSA, as a crude mimic of the PPA dianion, is effectively recognized by the urea binding site.

Some insight is provided by the MD simulations. Regarding template rebinding in absence of modifier, it is clear from the $\mathrm{H}$-bond analysis results (Table 3 ) that with respect to neutral templates, 1 will interact more strongly with PPA than with PSA. This agrees with the observed retention order in pure acetonitrile (Figure 6a). These experimental results are based on investigations of polymer system $\mathrm{P} 2$, prepared using higher levels of 1 and PMP (Table 2, Figure S15). Interestingly, the $\mathrm{H}$-bond analysis results (Table 3 ) revealed that increasing the amount of 1 promotes template interaction while barely 
affecting involvement of $\mathrm{PMPH}^{+}$. Further, increasing the degree of deprotonation of template reduces templatetemplate interactions, which is reasonable given the increased charge repulsion. Strong support for the formation of higher order complexes is offered by the RDF analysis. The degree of aggregation is noticeable when inspecting the simulated systems visually (Figures 7, S16, and S17). There is a markedly

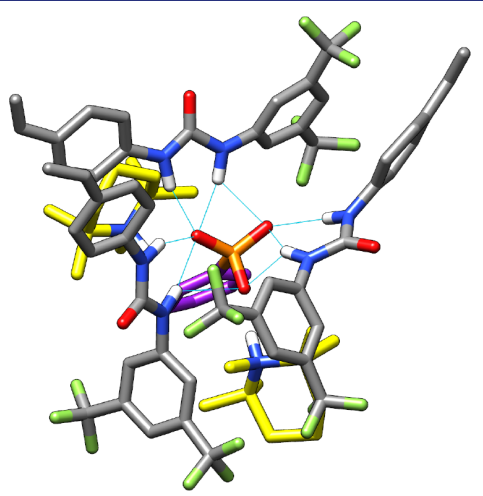

Figure 7. Example of an observed ternary complex in the P2C system, here involving three molecules of $\mathbf{1}$ (gray) engaged in H-bonding interactions with the template (PP2) (purple/magenta) and two non$\mathrm{H}$-bonding base molecules $\left(\mathrm{PMPH}^{+}\right)$(yellow). $\mathrm{H}$-bonds are indicated with dashed lines.

higher presence of $\mathrm{PMPH}^{+}$(2-fold) in the vicinity of PPA in the P2C system (Table 4) than in all the other systems. This supports the presence of ternary complexes and their possible influence on polymer structure and morphology (vide infra). ${ }^{50}$

Binding of Inorganic Phosphate and Sulfate in Water. Given the strong and switchable ion binding shown by the MIP in water poor media, our next goal was to investigate whether the polymers would cross-react with inorganic anions in buffer. Several synthetic receptors have been developed for this purpose to address various water processing or sensing applications, ${ }^{6}$ but so far only few receptors have shown effective anion recognition in water and even more rare are those functioning in high ionic strength media. ${ }^{1,51}$ A key problem is that both phosphate and sulfate are strongly hydrated in water (Table 1 ) which has a destabilizing effect on the interactions with the imprinted site.

To increase particle wettability, we prepared a new set of polymers as $\mathrm{P} 2 / \mathrm{P}_{\mathrm{N}} 2$ but using pentaerythritoltriacrylate (PETA) as a hydrophilic crosslinker. Ion binding was measured by conductometry in buffer $\mathrm{pH}=9.0$ where both anions carry a net 2 -fold negative charge (Figure 8 ). To our surprise, clear imprinting effects were observed with P7 exhibiting a consistently higher uptake of both phosphate and sulfate compared to nonimprinted $\mathrm{P}_{\mathrm{N}} 7$. In agreement with the Hofmeister series, describing the salt effect on protein solubility, phosphate is the most strongly bound ion followed by PPA, sulfate and PSA, the trend being weaker on $\mathrm{P}_{\mathrm{N}} 7$ compared to P7 (Table 6). The effect of imprinting appears clearly in the plots of the differential uptake in Figure $8 \mathrm{c}$ reflecting MIP binding corrected for binding to the NIP (assuming the latter to reflect the nonspecific binding contribution). Fitting these data with the Langmuir monosite
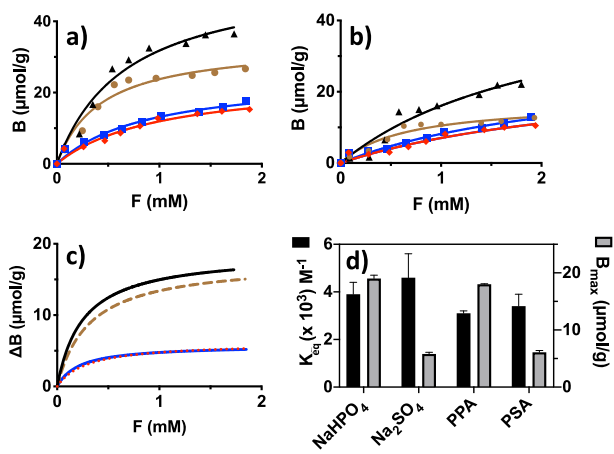

Figure 8. Equilibrium binding isotherms of $\mathrm{Na}_{2} \mathrm{HPO}_{4}$ (black curve, triangles), PPA (brown curve, circles), PSA (red curve, diamonds), and $\mathrm{Na}_{2} \mathrm{SO}_{4}$ (blue curve, squares) on polymer $\mathrm{P} 7$ (a) and $\mathrm{P}_{\mathrm{N}} 7$ (b) in $0.1 \mathrm{M}$ sodium bicarbonate buffer $\mathrm{pH}=9.0$ (c) Binding isotherms of P7 corrected for binding to the nonimprinted polymer $\mathrm{P}_{\mathrm{N}} 7$. (d) Equilibrium constants $\left(K_{\mathrm{eq}}\right.$, black bars) and binding capacities $\left(B_{\max }\right.$ gray bars) for the indicated anions interacting with $\mathrm{P} 7$ in buffer $\mathrm{pH}$ 9.0 The binding parameters were obtained by fitting of the corrected binding data in (c) to a Langmuir monosite binding model.

Table 6. Equilibrium Constants $\left(K_{\text {eq }}\right)$ and Binding Capacities $\left(B_{\max }\right)$ of PPA Imprinted Polymers ${ }^{a}$

\begin{tabular}{clccc} 
polymer & \multicolumn{1}{c}{ anion } & $K_{\text {eq }}\left(\times 10^{3} \mathrm{M}^{-1}\right)$ & $B_{\max }(\mu \mathrm{mol} / \mathrm{g})$ & $R^{2}$ \\
\hline $\mathrm{P7}$ & $\mathrm{NaHPO}_{4}$ & $1.5 \pm 0.3$ & $53 \pm 5$ & 0.973 \\
& $\mathrm{Na}_{2} \mathrm{SO}_{4}$ & $1.1 \pm 0.2$ & $25 \pm 3$ & 0.976 \\
& $\mathrm{PPA}$ & $2.2 \pm 0.6$ & $34 \pm 3$ & 0.966 \\
& $\mathrm{PSA}$ & $1.0 \pm 0.3$ & $24 \pm 3$ & 0.959 \\
$\mathrm{P}_{\mathrm{N}} 7$ & $\mathrm{NaHPO}_{4}$ & $0.5 \pm 0.2$ & $51 \pm 18$ & 0.940 \\
& $\mathrm{Na}_{2} \mathrm{SO}_{4}$ & $0.4 \pm 0.2$ & $30 \pm 8$ & 0.970 \\
& $\mathrm{PPA}$ & $1.5 \pm 0.5$ & $18 \pm 2$ & 0.950 \\
& $\mathrm{PSA}$ & $0.3 \pm 0.2$ & $27 \pm 11$ & 0.940 \\
$\mathrm{P}_{\text {corr }}$ & $\mathrm{NaHPO}_{4}$ & $3.9 \pm 0.5$ & $19 \pm 0.6$ & 0.990 \\
& $\mathrm{Na}_{2} \mathrm{SO}_{4}$ & $4.6 \pm 1.0$ & $5.8 \pm 0.3$ & 0.973 \\
& $\mathrm{PPA}$ & $3.1 \pm 0.1$ & $18 \pm 0.1$ & 0.999 \\
& $\mathrm{PSA}$ & $3.4 \pm 0.5$ & $6.1 \pm 0.3$ & 0.990
\end{tabular}

${ }^{a}$ The binding parameters were obtained by fitting of the binding data in Figure 8 to a Langmuir monosite binding model.

model resulted in the data shown in Figure 8d. Interestingly, in spite of the higher uptake of phosphate, sulfate binds with a slightly higher equilibrium constant $\left(K_{\mathrm{eq}}=4.6 \times 10^{3} \mathrm{M}^{-1}\right)$ than phosphate $\left(K_{\mathrm{eq}}=3.9 \times 10^{3} \mathrm{M}^{-1}\right)$. These values exceed the anion affinity of most reported neutral receptors with respect to anion binding in buffered media. ${ }^{4,5}$ To investigate a possible $\mathrm{pH}$ dependent anion preference, we also measured ion binding at lower $\mathrm{pH}$-values (Figure S8). Binding of both PPA and PSA decrease with decreasing $\mathrm{pH}$. Although an overturned binding preference could not be observed, the increase in the $B_{\mathrm{PSA}} / B_{\mathrm{PPA}}$ ratio shows that the preference for PPA decreases.

Investigation of Imprinting Mechanism. Having proven the binding performance of the anion imprinted receptors, further refinements require a fundamental understanding of the imprinting mechanism and the structural nature of the imprinted sites. An overlooked issue in molecular imprinting concerns the effect of template on the early stages of polymerization. ${ }^{52-55}$ Is the template capable of inducing a 


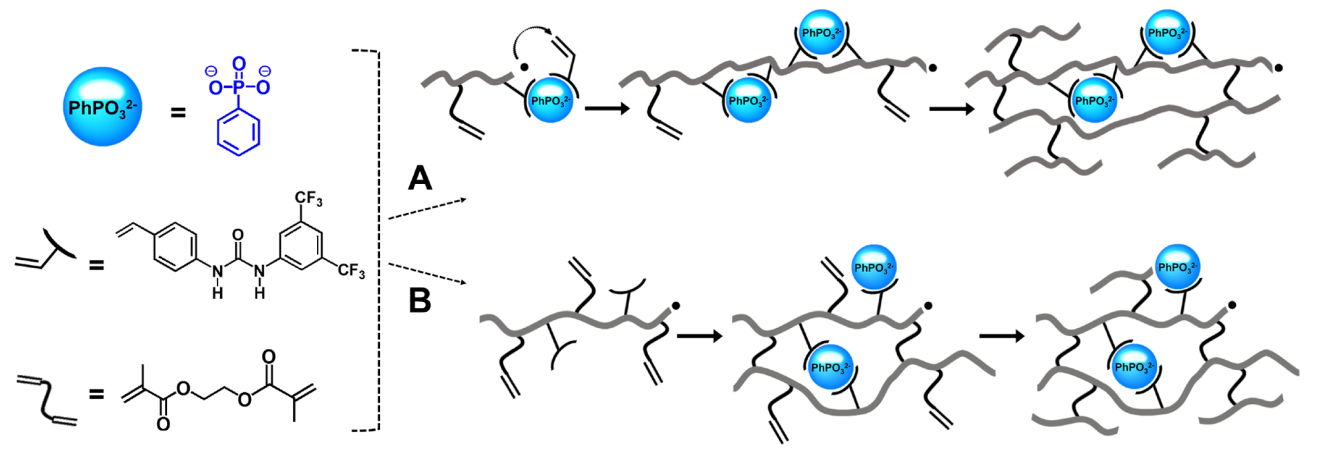

Figure 9. Alternative routes to the buildup of recognitive sites in network polymers. (A) Strong monomer-template interactions lead to template induced host monomer diads in the main chain that are subsequently stabilized by cross-linking. (B) Weak monomer-template interactions lead to sites formed by multidentade interactions involving preformed polymer chains. Counterions have been omitted for clarity.

preferred sequence or stereoregularity in the polymer main chain that may in turn influence its molecular recognition properties?

We raised this question in view of the ternary monomertemplate complexes anticipated to be present during polymerization, the presence of which finds strong support in results from the MD simulations (Tables 3 and 4 and Figures 7, S16, and S17). Such an arrangement may place the reactive double bonds of two monomers in close vicinity (see the left two molecules of $\mathbf{1}$ in Figure 7). One consequence of this arrangement may be a template induced incorporation of two urea groups juxtaposed in the same chain (Figure 9, route A) akin to the established template polymerization mechanism. ${ }^{55-58}$ The role of the template is here to bring two or more polymerizable groups close in space to achieve rate enhancement combined with stereo- and sequence- controlled monomer incorporation. The template can be covalently or noncovalently attached to the monomers and is cleaved off after polymerization to free up pendent functional groups. ${ }^{56}$ This results in high molecular weight copolymers with controlled tacticity and regularity.

To investigate whether monomer reactivity in our system is template controlled or the monomers are randomly incorporated, we used differential scanning calorimetry (DSC), NMR, and MALDI-TOF mass spectrometry to study the initial stages of polymerization. Cross-linked polymers P1 and P2 were compared with linear copolymers of $\mathbf{1}$ and methyl methacrylate (MMA). All polymers were prepared in absence or presence of template, the latter in the form of mono- or bis-TBA salts of PPA. First, we studied the curing process by DSC monitoring the heat generation upon double bond conversion. The onset and peak maxima temperatures of the curing exotherm were registered (Figures 10, S9, and S10), and the double bond conversion was estimated from the total heat generated per unsaturation divided by the literature value for the MMA double bond enthalpy $\left(\Delta H_{0}=13.1 \mathrm{kcal} / \mathrm{mol}\right)$ (Table S6). All copolymer curing systems experienced a lowering of the peak maxima temperatures upon added template, the effect being more pronounced for PPA.2TBA than for PPA.TBA (Figure 10a). This contrasted with the effect of template addition on the curing of MMA alone (Figure S10), where a slight increase in the onset temperature as well as a significantly lower
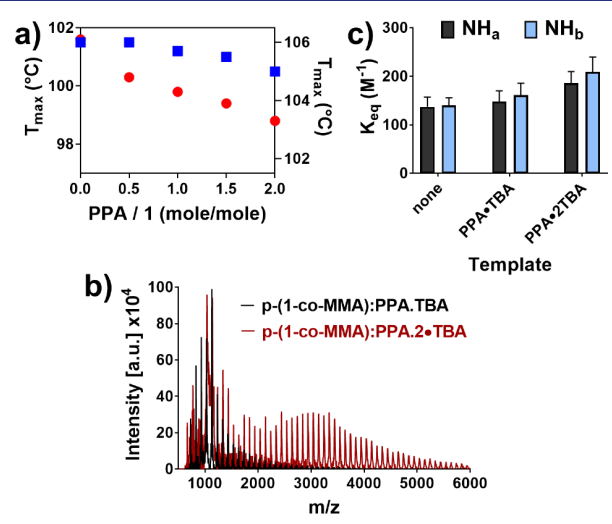

Figure 10. (a) Exotherm peak maxima temperatures upon curing of a mixture of 1 and MMA (1/MMA: $1 / 5 \mathrm{~mol} / \mathrm{mol})$ in the presence of PPA.2TBA (red circles) and PPA.TBA (blue squares). (b) MALDITOF-MS spectra of $\mathrm{p}$-1-co-MMA prepared in the presence of PPA TBA (black spectrum) and PPA.2TBA (red spectrum). (c) Equilibrium constants of PPA.2TBA binding to imprinted and nonimprinted p-(1-co-MMA). Data obtained from the binding curves in Figure S13 with fitting parameters listed in Table S7.

conversion was observed. This indicates that PPA acts as an inhibitor in absence of the host monomer. Overall, the template had no significant effect on the monomer conversion, which was $40-50 \%$ in all cases. We cautiously interpret these results as being due to a template assisted monomer incorporation with PPA.2TBA prearranging two molecules of 1 leading to a catalyzed propagation. The molecular weight distribution of $\mathrm{p}$-(1-co-MMA) determined by MALDI-TOFMS supports this explanation (Figures $10 \mathrm{~b}$ and S11). This technique provides detailed information on the molar mass distribution, end groups, and repeat units of linear polymers and in the tandem mode, confirmation of their structural identity. ${ }^{59}$

The polymers were isolated from the crude reaction mixtures by precipitation in methanol and deposited together 
Table 7. Properties of Linear Copolymers Prepared in the Presence of the Templates Indicated

\begin{tabular}{|c|c|c|c|c|c|c|}
\hline polymer & template & $\Delta T_{\max }{ }^{a}\left({ }^{\circ} \mathrm{C}\right)$ & $M_{\mathrm{w}}^{b}(\mathrm{~g} / \mathrm{mol})$ & $M_{\mathrm{n}}^{b}(\mathrm{~g} / \mathrm{mol})$ & $\mathrm{PDI}^{b}$ & $\mathrm{MMA} / 1^{c}$ \\
\hline p-MMA & PPA.2TBA & 0 & 1114 & 1025 & 1.09 & $\mathrm{n} / \mathrm{a}$ \\
\hline $\mathrm{p}-(1-c o-\mathrm{MMA})$ & none & $\mathrm{n} / \mathrm{a}$ & 1493 & 1174 & 1.27 & 1.74 \\
\hline $\mathrm{p}-(1-c o-\mathrm{MMA})$ & PPA.TBA & -1.0 & 1642 & 1319 & 1.24 & 2.36 \\
\hline $\mathrm{p}-(1-c o-\mathrm{MMA})$ & PPA.2TBA & -2.8 & 2700 & 1926 & 1.40 & 1.57 \\
\hline
\end{tabular}

${ }^{a}$ Change in exotherm peak maxima calculated from the data in Figures S9, S10, and 10a. ${ }^{b}$ Number $\left(M_{\mathrm{n}}\right)$ and weight $\left(M_{\mathrm{w}}\right)$ average molecular weights and polydispersity index (PDI) calculated as described in the SI. ${ }^{c}$ Calculated from the integrals of the signals corresponding to the $-\mathrm{OCH}_{3}$ protons of MMA $(\delta=3.6 \mathrm{ppm})$ and the aromatic protons $\mathrm{Hc}$ of $1(\delta=8.2 \mathrm{ppm})$.

with the MALDI matrix on the target plate followed by recording of the spectra. As seen in Figures $10 \mathrm{~b}$ and S11 the oligomer distributions were found in the ranges $\mathrm{m} / \mathrm{z} 100-$ 1500 p-MMA; 500-4000 p-(1-co-MMA); 700-5000 p-(1-coMMA) (template: PPA-TBA); 800-7000 p-(1-co-MMA) (template: PPA-2TBA), with the signal mass separations corresponding to the expected repeat units i.e. $100.1 \mathrm{~g} / \mathrm{mol}$. The corresponding number $\left(M_{\mathrm{n}}\right)$ and weight $\left(M_{\mathrm{w}}\right)$ average molecular weights and polydispersity index (PDI) are listed in Table 7.

From these results we conclude that polymerization in the presence of the doubly charged template leads to a pronounced increase in the average molecular weight of the oligomers, and a significant reduction in polydispersity, all in agreement with the anticipated mechanism.

Finally, we analyzed all polymers by ${ }^{1} \mathrm{H}$ NMR spectroscopy. This technique has a broad information value in polymer chemistry. ${ }^{60}$ From integral ratios of incorporated monomers, the copolymer composition can be estimated, and the technique can be used to estimate the number-average molecular weight, ${ }^{56,61}$ and polymer stereo- and sequenceregularity. ${ }^{55}$ Of particular relevance in our case is whether the signals from pendent urea groups are visible and if yes, whether their affinity vis-à-vis the template can be determined.

Figure $\mathrm{S} 12 \mathrm{a}$ shows a representative solution spectrum (DMSO- $\left.d_{6}\right)$ of $\mathrm{p}$-(1-co-MMA) prepared in absence of template. We gratefully noted that protons characteristic for both monomers were clearly visible, i.e., the urea protons $\mathrm{Ha}$ and $\mathrm{Hb}$ and the aromatic protons $\mathrm{Hc}$ of 1 and the $-\mathrm{OCH}_{3}$ protons of MMA. The integrals of the $\mathrm{Hc}$ and $-\mathrm{OCH}_{3}$ signals were used to estimate the stoichiometry of incorporated monomers. This is expressed as the ratio MMA/1 and the values are listed in Table 7 . For all polymers the ratios are lower than $5 / 1$ indicating an enhanced incorporation of monomer $\mathbf{1}$ in the chains. More interesting, however, is the low ratio observed for the template induced polymers, most notably for the polymer prepared in the presence of the doubly charged template. This agrees with the anticipated catalytic action of PPA.2TBA enhancing the reactivity of 1 . We thereafter monitored the complexation induced shifts of the urea protons upon addition of PPA.2TBA. As seen in Figure S12, titration was accompanied by pronounced downfield shifts and signal broadening which confirm the expected template-polymer interactions. The CIS plots for all polymers are seen in Figure S13 with the binding parameters listed in Table S7. The increase of the average equilibrium constants from $K_{\text {eq }}=139 \mathrm{M}^{-1}$ for the nontemplated polymer to $K_{\text {eq }}=$ $198 \mathrm{M}^{-1}$ for the templated polymer we ascribe to an enhanced number of main chain 1-1 diads, configured to bind the template via multiple $\mathrm{H}$-bonds.

We therefore conclude that a template memory can be induced by control of the monomer sequence in the polymer main chain alone. So, what is the role of the cross-linking agent? Judging from the equilibrium constants for adsorption, the affinity of the cross-linked P2 for PPA $\left(K_{\mathrm{eq}}=1.9 \times 10^{5}\right.$ $\mathrm{M}^{-1}$ ) exceeds by far that of the linear polymer. The contribution of cross-linking to the polymer affinity for the template is hence obvious. The importance of the cross-linking monomer is further highlighted in the results obtained from MD simulations. Looking at the neutral templated systems (PXN), cross-linker and $\mathbf{1}$ form $\mathrm{H}$-bonds between 30 and 50\% of the total simulation time (Table 3). Even in the PPA templated systems, extensive $\mathrm{H}$-bonding between the template and the cross-linking monomer is observed, $60-80 \%$. There is a high presence of cross-linker around templates and competition for access to functional groups on templates. The interactions are also stable, often more so than interactions between template and 1 or $\mathrm{PMP} / \mathrm{PMPH}^{+} . \mathrm{H}-$ bond interactions with template are lower in PSA systems than in PPA systems, even if the presence of cross-linker is similar. As observed in several other systems, ${ }^{44-48}$ the presence of cross-linker in close vicinity to the template is observed in prepolymerization mixtures and is observed to influence template-polymer rebinding.

\section{FINAL DISCUSSION}

Charge neutral receptors interacting with ligands via $\mathrm{H}$ bonding have for long been associated with poor compatibility with aqueous media. The presence of water and other protic solvents effectively disrupts the HG interactions in these systems. ${ }^{1}$ Some exceptions to this rule have recently been reported, although neutral receptors displaying affinity for phosphate and sulfate in pure water or buffer are still rare. Inspiration from naturally occurring receptors can be used to move forward. These display high affinity and selectivity in physiological conditions by shielding the anion from the surrounding solvent molecules and thereby freeing it to interact by $\mathrm{H}$-bonding in a nest like site. ${ }^{1,5}$ To mimic this principle, we have designed oxyanion imprinted polymers incorporating charge neutral urea groups in a hydrophobic scaffold. The polymers exhibit an unprecedented switchable anion binding behavior as well as strong ion affinity in buffered media comprising both phosphate and the strongly hydrated sulfate anion. This performance has a practical relevance in view of the broad need for robust anion binders. As we previously showed, this behavior may be exploited in bioseparations for fractionation of phosphorylated and sulfated peptides or saccharides. ${ }^{29}$ Another classical problem concerns sulfate separation from nitrate-rich radioactive mixtures which has been a long-term goal in nuclear waste remediation. Several synthetic receptors have been developed for this purpose but so far only few receptors offer effective anion recognition in water and even more rare are those functioning in high ionic strength media. Through further improvements of 
the imprinted receptors, such as incorporation of dual receptors $^{62,63}$ or other scaffolds, we hope to sufficiently boost affinity and selectivity for the MIPs to offer a viable alternative host for these purposes.

We also provide detailed molecular level insight into the anion recognitive sites. Comparing stoichiometric imprinting based on $1: 1$ or $1: 2$ phosphate anion-urea monomer ratios shows the superiority of the ternary complex imprinting to generate high fidelity binding sites. In spite of the stoichiometric complexation as proven by ${ }^{1} \mathrm{H}$ NMR $2 \mathrm{D}$ NOESY and CIS plots, the imprinting efficiency did not exceed $30 \%$ when counting the high affinity sites. This is likely related to the amorphous nature of this class of MIPs, ${ }^{64}$ supported by the heterogeneity of observed complexes in these mixtures (Figures S16 and S17). MIPs prepared by free radical polymerization belong to the thermoset class of materials characterized by extensive and irreversible cross-linking and insoluble end products. The heterogeneity of these materials ranges from the molecular level to the micro scale which precludes molecular level insights into the structural and dynamic features of the binding sites. This stands in contrast to the range of available tools for characterizing biomacromolecules. Nevertheless, reports focusing on well characterized monomer template systems employing advanced techniques such as solid state NMR with isotopically enriched templates, $^{65-68}$ infrared $^{69,70}$ or fluorescence spectroscopy $^{42,71,72}$ have increased our knowledge in this regard. As discussed above, the propagation rate versus the on/off rate of the template-monomer interaction is an indicator for the templates ability to induce repeat units in the main chain. ${ }^{73}$ Very few studies on how the template influences the initial stages of the polymerization have been reported. ${ }^{54,55}$ This warrants further studies in this regard. As we have shown in this report, a solution binding constant for the host monomer template interaction exceeding $7000 \mathrm{M}^{-1}$ is sufficient for achieving this. This contrasts with situations where the rate of propagation is slow with respect to the on/off rate. Here the template will have time to dissociate before the next monomer addition. In this case, imprinting follows the mechanism outlined in Figure 9b where preformed polymer chains are conformationally stabilized by the template via multidentate interactions. Although this is likely the dominating mechanism in most noncovalent imprinting systems, ${ }^{16-20}$ studies of corresponding linear polymers can provide further important insights into the imprinting process.

\section{ASSOCIATED CONTENT}

(5) Supporting Information

The Supporting Information is available free of charge at https://pubs.acs.org/doi/10.1021/jacs.0c00707.

Experimental section outlining synthetic procedures, characterization techniques and data evaluation and dynamic simulations, and supporting tables and graphics (PDF)

\section{AUTHOR INFORMATION}

\section{Corresponding Author}

Börje Sellergren - Department of Biomedical Sciences, Faculty of Health and Society, Malmö University, 20506 Malmö, Sweden; Faculty of Chemistry, Technical University of Dortmund, 44227 Dortmund, Germany; $\odot$ orcid.org/00000002-2392-3305; Email: borje.sellegren@mau.se

\section{Authors}

Sudhirkumar Shinde - Department of Biomedical Sciences, Faculty of Health and Society, Malmö University, 20506 Malmö, Sweden; Faculty of Chemistry, Technical University of Dortmund, 44227 Dortmund, Germany; $\odot$ orcid.org/00000001-9460-0936

Anil Incel - Department of Biomedical Sciences, Faculty of Health and Society, Malmö University, 20506 Malmö, Sweden

Mona Mansour - Department of Biomedical Sciences, Faculty of Health and Society, Malmö University, 20506 Malmö, Sweden

Gustaf D. Olsson - Bioorganic \& Biophysical Chemistry Laboratory, Linneaus University Center for Biomaterials Chemistry, Department of Chemistry \& Biomedical Sciences, Linnaeus University, 39182 Kalmar, Sweden

Ian A. Nicholls - Bioorganic \& Biophysical Chemistry Laboratory, Linneaus University Center for Biomaterials Chemistry, Department of Chemistry \& Biomedical Sciences, Linnaeus University, 39182 Kalmar, Sweden; ๑ orcid.org/ 0000-0002-0407-6542

Cem Esen - Faculty of Chemistry, Technical University of Dortmund, 44227 Dortmund, Germany

Javier Urraca - Faculty of Chemistry, Technical University of Dortmund, 44227 Dortmund, Germany

Complete contact information is available at: https://pubs.acs.org/10.1021/jacs.0c00707

\section{Author Contributions}

These authors contributed equally. The manuscript was written through contributions of all authors. All authors have given approval to the final version of the manuscript.

\section{Funding}

Marie Skłodowska-Curie Actions PITN-GA-2010-264699 and H2020-MSCA-ETN-2016, 722171; Swedish Vetenskapsrådet (VR) under grant number 2014-3794; Sweden KnowledgeFoundation (KKS) for research under project number 20150086. Deutsche Forschungsgemeinsc haft (DFG) (Se 777/9-1).

\section{Notes}

The authors declare no competing financial interest.

\section{ACKNOWLEDGMENTS}

We gratefully acknowledge financial support from the Marie Skłodowska-Curie Actions (PITN-GA-2010-264699 and H2020-MSCA-ITN-2016, 722171), Vetenskapsrådet (VR) under grant number 2014-3794, the Deutsche Forschungsgemeinschaft (DFG) under grant number Se 777/9-1 and the Sweden and the Sweden Knowledge-Foundation (KKS) for research under project number 20150086. S.S. gratefully acknowledges a Matching Funds fellowship from the Deutsche Akademische Austauschdienst (DAAD). C.E. is grateful for the postdoctoral research fellowship from the Council of Higher Education in Turkey (YÖK) and for the visiting academic funding from the German Academic Exchange Service (DAAD).

\section{REFERENCES}

(1) Langton, M. J.; Serpell, C. J.; Beer, P. D. Anion Recognition in Water: Recent Advances from a Supramolecular and Macromolecular Perspective. Angew. Chem., Int. Ed. 2016, 55 (6), 1974-1987.

(2) Kubik, S. Supramolecular Chemistry in Water; Wiley-VCH, 2019; p 244. 
(3) Heymann, J. J.; Weaver, K. D.; Mietzner, T. A.; Crumbliss, A. L. Sulfate as a Synergistic Anion Facilitating Iron Binding by the Bacterial Transferrin FbpA: The Origins and Effects of Anion Promiscuity. J. Am. Chem. Soc. 2007, 129 (31), 9704-9712.

(4) Hargrove, A. E.; Nieto, S.; Zhang, T.; Sessler, J. L.; Anslyn, E. V. Artificial Receptors for the Recognition of Phosphorylated Molecules. Chem. Rev. 2011, 111 (11), 6603-6782.

(5) Ravikumar, I.; Ghosh, P. Recognition and separation of sulfate anions. Chem. Soc. Rev. 2012, 41 (8), 3077-3098.

(6) Fowler, C. J.; Haverlock, T. J.; Moyer, B. A.; Shriver, J. A.; Gross, D. E.; Marquez, M.; Sessler, J. L.; Hossain, M. A.; Bowman-James, K. Enhanced anion exchange for selective sulfate extraction: overcoming the Hofmeister bias. J. Am. Chem. Soc. 2008, 130 (44), 14386-14387.

(7) Sommer, F.; Kubik, S. Anion binding of a neutral bis(cyclopeptide) in water-methanol mixtures containing up to $95 \%$ water. Org. Biomol. Chem. 2014, 12 (44), 8851-8860.

(8) Liu, W.-X.; Jiang, Y.-B. Intramolecular Hydrogen Bonding and Anion Binding of N-Benzamido- $\mathrm{N}^{\prime}$-benzoylthioureas. J. Org. Chem. 2008, 73 (3), 1124-1127.

(9) Duke, R. M.; O’Brien, J. E.; McCabe, T.; Gunnlaugsson, T. Colorimetric sensing of anions in aqueous solution using a charge neutral, cleft-like, amidothiourea receptor: tilting the balance between hydrogen bonding and deprotonation in anion recognition. Org. Biomol. Chem. 2008, 6 (22), 4089-4092.

(10) Ravikumar, I.; Lakshminarayanan, P. S.; Arunachalam, M.; Suresh, E.; Ghosh, P. Anion complexation of a pentafluorophenylsubstituted tripodal urea receptor in solution and the solid state: selectivity toward phosphate. Dalton Transactions 2009, No. 21, 4160-4168.

(11) Emami Khansari, M.; Mirchi, A.; Pramanik, A.; Johnson, C.; Leszczynski, J.; Hossain, M. Remarkable hexafunctional anion receptor with operational urea-based inner cleft and thiourea-based outer cleft: Novel design with high-efficiency for sulfate binding. Sci. Rep. 2017, 7 DOI: 10.1038/s41598-017-05831-x.

(12) Jin, C.; Zhang, M.; Wu, L.; Guan, Y.; Pan, Y.; Jiang, J.; Lin, C.; Wang, L. Squaramide-based tripodal receptors for selective recognition of sulfate anion. Chem. Commun. 2013, 49 (20), 2025-2027.

(13) Busschaert, N.; Elmes, R. B. P.; Czech, D. D.; Wu, X.; Kirby, I. L.; Peck, E. M.; Hendzel, K. D.; Shaw, S. K.; Chan, B.; Smith, B. D.; Jolliffe, K. A.; Gale, P. A. Thiosquaramides: $\mathrm{pH}$ switchable anion transporters. Chemical Science 2014, 5 (9), 3617-3626.

(14) Qin, L.; Wright, J. R.; Lane, J. D. E.; Berry, S. N.; Elmes, R. B. P.; Jolliffe, K. A. Receptors for sulfate that function across a wide $\mathrm{pH}$ range in mixed aqueous-DMSO media. Chem. Commun. 2019, 55 (82), 12312-12315

(15) Whitcombe, M. J.; Kirsch, N.; Nicholls, I. A. Molecular imprinting science and technology: a survey of the literature for the years 2004-2011. J. Mol. Recognit. 2014, 27 (6), 297-401.

(16) Chen, L.; Wang, X.; Lu, W.; Wu, X.; Li, J. Molecular imprinting: perspectives and applications. Chem. Soc. Rev. 2016, 45 (8), 2137-2211.

(17) Haupt, K.; Ayela, C. Molecular Imprinting; Springer, 2012.

(18) Sellergren, B.; Hall, A. J., Molecularly Imprinted Polymers. In Supramolecular Chemistry: from Molecules to Nanomaterials; Steed, J. W., Gale, P. A., Eds.; John Wiley \& Sons, Ltd: Chichester, UK, 2012; pp 3255-3282.

(19) Takeuchi, T.; Hayashi, T.; Ichikawa, S.; Kaji, A.; Masui, M.; Matsumoto, H.; Sasao, R. Molecularly Imprinted Tailor-Made Functional Polymer Receptors for Highly Sensitive and Selective Separation and Detection of Target Molecules. Chromatography 2016, 37 (2), 43-64.

(20) Cheong, W. J.; Yang, S. H.; Ali, F. Molecular imprinted polymers for separation science: A review of reviews. J. Sep. Sci. 2013, 36 (3), 609-628.

(21) Wierzbicka, C.; Liu, M.; Bauer, D.; Irgum, K.; Sellergren, B. Cationic $\mathrm{pTyr} / \mathrm{pSer}$. imprinted polymers based on a bis-imidazolium host monomer: phosphopeptide recognition in aqueous buffers demonstrated by $\mu$-liquid chromatography and monolithic columns. J. Mater. Chem. B 2017, 5 (5), 953-960.
(22) Sulc, R.; Szekely, G.; Shinde, S.; Wierzbicka, C.; Vilela, F.; Bauer, D.; Sellergren, B. Phospholipid imprinted polymers as selective endotoxin scavengers. Sci. Rep. 2017, 7, 44299.

(23) Liu, M.; Torsetnes, S. B.; Wierzbicka, C.; Jensen, O. N.; Sellergren, B.; Irgum, K. Selective Enrichment of Phosphorylated Peptides by Monolithic Polymers Surface Imprinted with bisImidazolium Moieties by UV-Initiated Cryopolymerization. Anal. Chem. 2019, 91 (15), 10188-10196.

(24) Wulff, G.; Gross, T.; Schönfeld, R. Enzyme models based on molecularly imprinted polymers with strong esterase activity. Angew. Chem., Int. Ed. Engl. 1997, 36, 1962-9164.

(25) Wulff, G.; Knorr, K. Stoichiometric imprinting. Bioseparation 2001, 10, 257.

(26) Cutivet, A.; Schembri, C.; Kovensky, J.; Haupt, K. Molecularly Imprinted Microgels as Enzyme Inhibitors. J. Am. Chem. Soc. 2009, 131 (41), 14699-14702.

(27) Ambrosini, S.; Beyazit, S.; Haupt, K.; Tse Sum Bui, B. Solidphase synthesis of molecularly imprinted nanoparticles for protein recognition. Chem. Commun. 2013, 49 (60), 6746-6748.

(28) Emgenbroich, M.; Borrelli, C.; Shinde, S.; Lazraq, I.; Vilela, F.; Hall, A. J.; Oxelbark, J.; De Lorenzi, E.; Courtois, J.; Simanova, A.; Verhage, J.; Irgum, K.; Karim, K.; Sellergren, B. A PhosphotyrosineImprinted Polymer Receptor for the Recognition of Tyrosine Phosphorylated Peptides. Chem. - Eur. J. 2008, 14, 9516-9529.

(29) Shinde, S.; Bunschoten, A.; Kruijtzer, J. A. W.; Liskamp, R. M. J.; Sellergren, B. Imprinted Polymers Displaying High Affinity for Sulfated Protein Fragments. Angew. Chem., Int. Ed. 2012, 51 (33), 8326-8329.

(30) Wan, W.; Biyikal, M.; Wagner, R.; Sellergren, B.; Rurack, K. Fluorescent Sensory Microparticles that "Light-up" Consisting of a Silica Core and a Molecularly Imprinted Polymer (MIP) Shell. Angew. Chem., Int. Ed. 2013, 52 (27), 7023-7027.

(31) Shinde, S.; El-Schich, Z.; Malakpour, A.; Wan, W.; Dizeyi, N.; Mohammadi, R.; Rurack, K.; Gjörloff Wingren, A.; Sellergren, B. Sialic acid imprinted fluorescent core-shell particles for selective labeling of cell surface glycans. J. Am. Chem. Soc. 2015, 137, 13908-13912.

(32) Kugimiya, A.; Takei, H. Selectivity and recovery performance of phosphate-selective molecularly imprinted polymer. Anal. Chim. Acta 2008, 606 (2), 252-256.

(33) Manesiotis, P.; Riley, A.; Bollen, B. Polymerisable squaramide receptors for anion binding and sensing. J. Mater. Chem. C 2014, 2 (42), 8990-8995.

(34) Lübke, C.; Lübke, M.; Whitcombe, M. J.; Vulfson, E. N. Imprinted polymers prepared with stoichiometric template-monomer complexes: Efficient binding of ampicillin from aqueous solutions. Macromolecules 2000, 33, 5098-5105.

(35) Manesiotis, P.; Hall, A. J.; Emgenbroich, M.; Quaglia, M.; De Lorenzi, E.; Sellergren, B. An enantioselective imprinted receptor for Z-glutamate exhibiting a binding induced color change. Chem. Commun. 2004, 2278-2279.

(36) Urraca, J. L.; Hall, A. J.; Moreno-Bondi, M. C.; Sellergren, B. A Stoichiometric Molecularly Imprinted Polymer for the Class-Selective Recognition of Antibiotics in Aqueous Media. Angew. Chem., Int. Ed. 2006, 45, 5158-5161.

(37) Urraca, J. L.; Aureliano, C. S. A.; Schillinger, E.; Esselmann, H.; Wiltfang, J.; Sellergren, B. Polymeric Complements to the Alzheimer's Disease Biomarker $\beta$-Amyloid Isoforms $\mathrm{A} \beta 1-40$ and $\mathrm{A} \beta 1-42$ for Blood Serum Analysis under Denaturing Conditions. J. Am. Chem. Soc. 2011, 133 (24), 9220-9223.

(38) Fan, E.; Van Arman, S. A.; Kincaid, S.; Hamilton, A. D. Moleclar recognition: Hydrogen bonding receptors that function in highly competitive solvents. J. Am. Chem. Soc. 1993, 115, 369-370.

(39) Gomez, D. E.; Fabbrizzi, L.; Licchelli, M.; Monzani, E. Urea vs. thiourea in anion recognition. Org. Biomol. Chem. 2005, 3, 14951500 .

(40) Pérez-Casas, C.; Yatsimirsky, A. K. Detailing Hydrogen Bonding and Deprotonation Equilibria between Anions and Urea/ Thiourea Derivatives. J. Org. Chem. 2008, 73 (6), 2275-2284. 
(41) Wan, W.; Descalzo, A. B.; Shinde, S.; Weißhoff, H.; Orellana, G.; Sellergren, B.; Rurack, K. Ratiometric Fluorescence Detection of Phosphorylated Amino Acids Through Excited-State Proton Transfer by Using Molecularly Imprinted Polymer (MIP) Recognition Nanolayers. Chem. - Eur. J. 2017, 23 (63), 15974-15983.

(42) Wagner, S.; Zapata, C.; Wan, W.; Gawlitza, K.; Weber, M.; Rurack, K. Role of Counterions in Molecularly Imprinted Polymers for Anionic Species. Langmuir 2018, 34 (23), 6963-6975.

(43) Kelly, T. R.; Kim, M. H. Relative binding affinity of carboxylate and its isoteres: Nitro, phosphoate, phosphonate, sulfonate and $\mathrm{d}$ lactone. J. Am. Chem. Soc. 1994, 116, 7072-7080.

(44) Boiocchi, M.; Del Boca, L.; Gómez, D. E.; Fabbrizzi, L.; Licchelli, M.; Monzani, E. Nature of Urea-Fluoride Interaction: Incipient and Definitive Proton Transfer. J. Am. Chem. Soc. 2004, 126 (50), 16507-16514.

(45) Karlsson, B. C.; O’Mahony, J.; Karlsson, J. G.; Bengtsson, H.; Eriksson, L. A.; Nicholls, I. A. Structure and dynamics of monomertemplate complexation: an explanation for molecularly imprinted polymer recognition site heterogeneity. J. Am. Chem. Soc. 2009, 131 (37), 13297-304.

(46) Shoravi, S.; Olsson, G. D.; Karlsson, B. C.; Nicholls, I. A. On the influence of crosslinker on template complexation in molecularly imprinted polymers: a computational study of prepolymerization mixture events with correlations to template-polymer recognition behavior and NMR spectroscopic studies. Int. J. Mol. Sci. 2014, 15 (6), 10622-34.

(47) Golker, K.; Karlsson, B. C. G.; Olsson, G. D.; Rosengren, A. M.; Nicholls, I. A. Influence of Composition and Morphology on Template Recognition in Molecularly Imprinted Polymers. Macromolecules 2013, 46 (4), 1408-1414.

(48) Olsson, G. D.; Niedergall, K.; Bach, M.; Karlsson, B. C. G.; Tovar, G.; Nicholls, I. A. Simulation of imprinted emulsion prepolymerization mixtures. Polym. J. 2015, 47 (12), 827-830.

(49) Pramanik, A.; Thompson, B.; Hayes, T.; Tucker, K.; Powell, D. R.; Bonnesen, P. V.; Ellis, E. D.; Lee, K. S.; Yu, H.; Hossain, M. A. Seven-coordinate anion complex with a tren-based urea: Binding discrepancy of hydrogen sulfate in solid and solution states. Org. Biomol. Chem. 2011, 9 (12), 4444-4447.

(50) Shimizu, K. D. Binding isotherms. In Molecularly Imprinted Materials: Science and Technology 2004, 419-434.

(51) Busschaert, N.; Caltagirone, C.; Van Rossom, W.; Gale, P. A. Applications of Supramolecular Anion Recognition. Chem. Rev. 2015, 115 (15), 8038-8155.

(52) Vaughan, A. D.; Sizemore, S. P.; Byrne, M. E. Enhancing molecularly imprinted polymer binding properties via controlled/ living radical polymerization and reaction analysis. Polymer 2007, 48 (1), 74-81.

(53) Yungerman, I.; Srebnik, S. Factors Contributing to Binding-Site Imperfections in Imprinted Polymers. Chem. Mater. 2006, 18 (3), $657-663$.

(54) Lanza, F.; Ruther, M.; Hall, A.J.; Dauwe, C.; Sellergren, B. Studies on the Process of formation, Nature and Stability of Binding Sites in Molecularly Imprinted Polymers. Mater. Res. Soc. Symp. Proc. 2002, 723, 1-11.

(55) Wulff, G.; Hohn, J. Chirality of polyvinyl compounds. 2. An asymmetric copolymerization. Macromolecules 1982, 15 (5), 125561.

(56) Pasini, D.; Takeuchi, D. Cyclopolymerizations: Synthetic Tools for the Precision Synthesis of Macromolecular Architectures. Chem. Rev. 2018, 118 (18), 8983-9057.

(57) Połowiński, S. Template polymerisation and co-polymerisation. Prog. Polym. Sci. 2002, 27 (3), 537-577.

(58) Chapiro, A. Auto-acceleration in free radical polymerizations caused by oriented monomer-polymer association complexes. Pure Appl. Chem. 1981, 53, 643-655.

(59) Giordanengo, R.; Viel, S.; Hidalgo, M.; Allard-Breton, B.; Thévand, A.; Charles, L. Analytical strategy for the molecular weight determination of random copolymers of poly(methyl methacrylate) and poly(methacrylic acid). J. Am. Soc. Mass Spectrom. 2010, 21 (6), 1075-1085.

(60) Diehl, B., Chapter 2 - NMR Applications for Polymer Characterisation. In NMR Spectroscopy in Pharmaceutical Analysis; Holzgrabe, U., Wawer, I., Diehl, B., Eds.; Elsevier: Amsterdam, 2008; pp $157-180$.

(61) Terashima, T.; Kawabe, M.; Miyabara, Y.; Yoda, H.; Sawamoto, M. Polymeric pseudo-crown ether for cation recognition via cation template-assisted cyclopolymerization. Nat. Commun. 2013, 4 (1), 2321.

(62) Kim, S. K.; Sessler, J. L. Ion pair receptors. Chem. Soc. Rev. 2010, 39 (10), 3784-3809.

(63) Shinde, S.; Mansour, M.; Incel, A.; Mavliutova, L.; Wierzbicka, C.; Sellergren, B. High salt compatible oxyanion receptors by dual ion imprinting. Chem. Sci. 2020, 11 (16), 4246-4250.

(64) Sellergren, B.; Shea, K. J. On the influence of polymer morphology on the ability of imprinted polymers to separate enantiomers. J. Chromatogr. 1993, 635, 31.

(65) Shea, K. J.; Sasaki, D. Y. An analysis of small molecule binding to functionalized synthetic polymers by $13 \mathrm{C} \mathrm{CP/MAS} \mathrm{NMR} \mathrm{and} \mathrm{FT-}$ IR spectroscopy. J. Am. Chem. Soc. 1991, 113, 4109-4120.

(66) Shea, K. J.; Sasaki, D. Y. On the Control of Microenvironment Shape of Functionalized Network Polymers Prepared by Template Polymerization. J. Am. Chem. Soc. 1989, 111, 3442-3444.

(67) Skogsberg, U.; Meyer, C.; Rehbein, J.; Fischer, G.; Schauff, S.; Welsch, N.; Albert, K.; Hall, A. J.; Sellergren, B. A solid-state and suspended-state magic angle spinning nuclear magnetic resonance spectroscopic investigation of a 9-ethyladenine molecularly imprinted polymer. Polymer 2007, 48, 229-238.

(68) O’Mahony, J.; Molinelli, A.; Nolan, K.; Smyth, M. R.; Mizaikoff, B. Anatomy of a successful imprint: Analysing the recognition mechanisms of a molecularly imprinted polymer for quercetin. Biosens. Bioelectron. 2006, 21 (7), 1383-1392.

(69) Molinelli, A.; O'Mahony, J.; Nolan, K.; Smyth, M. R.; Jakusch, M.; Mizaikoff, B. Analyzing the Mechanisms of Selectivity in Biomimetic Self-Assemblies via IR and NMR Spectroscopy of Prepolymerization Solutions and Molecular Dynamics Simulations. Anal. Chem. 2005, 77 (16), 5196-5204.

(70) Kostrewa, S.; Emgenbroich, M.; Klockow, D.; Wulff, G. Surface-enhanced Raman scattering on molecularly imprinted polymers in water. Macromol. Chem. Phys. 2003, 204 (3), 481-487.

(71) Shea, K. J.; Sasaki, D. Y.; Stoddard, G. J. Fluorescence probes for evaluating chain solvation in network polymers. An analysis of the solvatochromic shift of the dansyl probe in macroporous styrenedivinylbenzene and styrene-diisopropenylbenzene copolymers. Macromolecules 1989, 22, 1722-1730.

(72) Svenson, J.; Andersson, H. S.; Piletsky, S. A.; Nicholls, I. A. Spectroscopic studies of the molecular imprinting self-assembly process. J. Mol. Recognit. 1998, 11 (1-6), 83-86.

(73) Sellergren, B.; Hall, A. J. Fundamental aspects on the synthesis and characterization of imprinted network polymers. In Molecularly Imprinted Polymers. Man-Made Mimics of Antibodies and Their Applications in Analytical Chemistry; Sellergren, B., Ed.; Elesevier Science B.V.: Amsterdam, 2001; Vol. 23, pp 21-57. 

II 


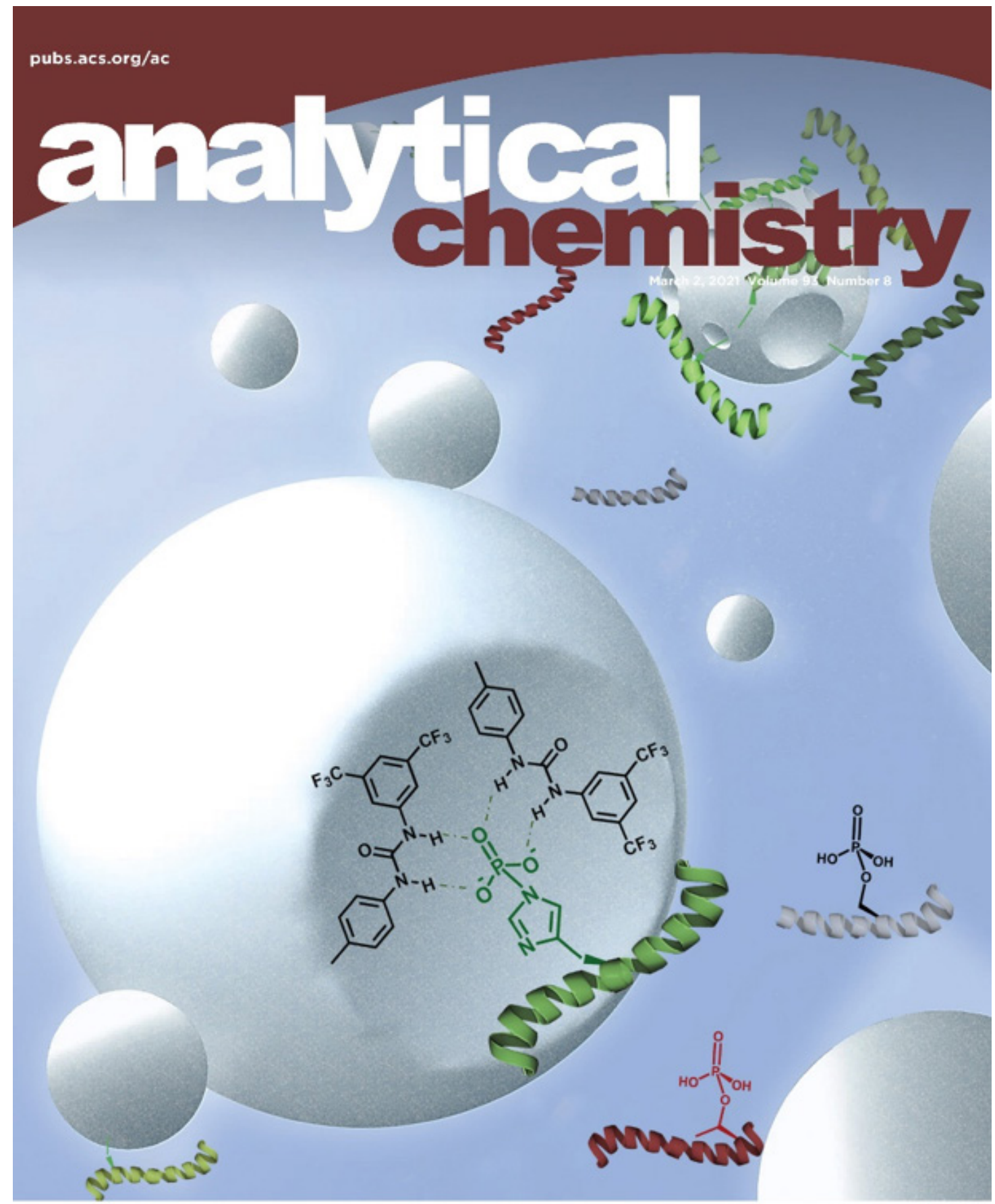




\section{Selective Enrichment of Histidine Phosphorylated Peptides Using Molecularly Imprinted Polymers}

Anıl Incel, Ignacio Arribas Díez, Celina Wierzbicka, Katarzyna Gajoch, Ole N. Jensen, and Börje Sellergren*

\section{INTRODUCTION}

Protein phosphorylation is one of the most widely studied and best-understood post-translational modifications (PTMs). It is involved in the regulation of vital biological processes, including cellular signal transduction. ${ }^{1}$ Although phosphorylation can occur on at least nine different amino acid residues, i.e., serine, threonine, tyrosine, histidine, lysine, arginine, aspartate, glutamate, and cysteine, the vast majority of phosphoproteomics research is focused on the phosphorylation of the former three residues. In recent years, however, the role of histidine phosphorylation (pHis) has gained attention. This modification is well known to be involved in two-component protein-signaling networks in prokaryotes and lower eukaryotes $^{2-4}$ and is also found in mammals and implicated in certain human disease states. ${ }^{5-}$

Phosphohistidine can exist in two isomeric forms, i.e., $\pi$ pHis and $\tau$-pHis, and the doubly phosphorylated $\tau, \pi$-pHis (Figure 1), of which the former two exist in vivo. ${ }^{8}$ It has been estimated that histidine phosphorylation in eukaryotes accounts for $6 \%$ of the total protein phosphorylation ${ }^{9}$ and is more abundant than the phosphotyrosine (pTyr) modification. Nonetheless, little is known about the biological roles of pHis as compared to phosphoester modifications, i.e., phosphoserine (pSer), phosphothreonine (pThr), and phosphotyrosine. This is due to significant technical challenges in the enrichment and<smiles>NC(Cc1cncn1P(=O)(O)O)C(=O)O</smiles><smiles>NC(Cc1cn(S(=O)(=O)O)cn1)C(=O)O</smiles><smiles></smiles><smiles>C=Cc1ccc(NC(=O)Nc2cc(C(F)(F)F)cc(C(F)(F)F)c2)cc1</smiles><smiles>CCOC(=O)COCCOC(=O)C(Cn1cc(P(O)(O)(O)O)nn1)NC(=O)OCC1c2ccccc2-c2ccccc21</smiles>

Figure 1. Structures of $\mathrm{pHis}$ isomers, neutral-urea-based functional monomer 1, and template Fmoc-pTza-OEt.

Received: October 22, 2020

Accepted: February 4, 2021

Published: February 16, 2021 
detection of pHis in complex biological samples. Unlike the phosphoester amino acids, the phosphoryl group of $\mathrm{pHis}$ is attached to a nitrogen atom, making it a phosphoramidate.

The high-energy phosphoramidate bond is susceptible to hydrolysis in acidic environments and $\mathrm{pHis}$ exhibits a unique stability profile. ${ }^{10}$ While O-phosphohydroxy amino acids (pSer, pThr, pTyr) are stable in acidic media and labile in alkali environment (pSer, pThr), the phosphoramidates display an inverse stability profile, i.e., they are base-stable and acidlabile. ${ }^{11}$ Most of the proteomics methods used for phosphopeptide (p-peptide) enrichment and detection involve acidic conditions during sample preparation and thus fail to preserve the pHis modification. Therefore, dedicated bioanalytical methods and protocols are needed to study pHis in proteins. Several techniques for the enrichment, detection, and analysis of pHis modification have been reported to date and reviewed elsewhere. ${ }^{8,12-14}$ Mild enrichment methods, ideally avoiding acidic treatment of the sample, are of critical importance for the development of pHis detection methods. Metal oxide affinity chromatography (MOAC, including $\mathrm{TiO}_{2}$ ) and immobilized metal ion affinity chromatography (IMAC), which are commonly used for enrichment of phosphorylated peptides, are in principle not suitable for enrichment of $\mathrm{pHis}$ since they employ acidic conditions. ${ }^{15}$ Nevertheless, an attempt to enrich pHis peptides using $\mathrm{Cu}^{2+}$-IMAC with mild acidic conditions and $\mathrm{Fe}^{3+}$-IMAC has been reported before. $^{16-20}$ Recent efforts to produce phosphohistidine antibodies proved successful owing to the development of stable phosphohistidine analogues. ${ }^{21-24}$ Notwithstanding these advances, the drawbacks of antibodies include high production cost, poor reproducibility, and limited stability.

We report on a method for enrichment of pHis peptides using custom-made molecularly imprinted polymers (MIPs). In this proof-of-concept study, we demonstrate two approaches for selective enrichment of pHis peptides. In the first chemoaffinity approach, a pHis-MIP (MIP1) is used to enrich pHis peptides with moderate pHis discrimination. Subsequently, base-labile phosphoserine (pSer) and phosphothreonine (pThr) peptides are depleted from the sample by treatment with $\mathrm{Ba}(\mathrm{OH})_{2}$, resulting in a method obviating any acid-based sample treatment. The second method is based on newgeneration pHis-MIPs (MIP2 and MIP3), which was finetuned in two steps with respect to binding site design and pore size distribution. The optimized pHis-MIP (MIP3) enabled enrichment of $\mathrm{pHis}$ peptides from tryptic digests of protein mixtures prior to analysis and sequencing by liquid chromatography-mass spectrometry (LC-MS).

\section{EXPERIMENTAL SECTION}

Preparation of pHis-Imprinted Polymer Particles by Crushing and Sieving. Fmoc-pTza-OEt (see Figures S1 and 1) $(0.04 \mathrm{mmol})$ was dispersed in dry $\mathrm{MeCN}(0.43 \mathrm{~mL})$ in a 2 $\mathrm{mL}$ screw-capped reaction vial equipped with a rubber septum. This was followed by addition of 1,2,2,6,6-pentamethylpiperidine (PMP, $0.08 \mathrm{mmol})(\mathrm{MIP} 1 / \mathrm{NIP} 1)$ or tetrabutylammonium hydroxide (TBA.OH) (MIP2/NIP2), functional monomer 1 (Figure 1) $(0.08 \mathrm{mmol})$, acrylamide $(0.08 \mathrm{mmol})$, pentaerythritol triacrylate (PETA, $1.09 \mathrm{mmol}$ ), and finally the thermal initiator ABDV ( $1 \% \mathrm{w} / \mathrm{w}$ of total monomer mass). The solution was cooled to $0{ }^{\circ} \mathrm{C}$ and purged with a flow of nitrogen gas for $15 \mathrm{~min}$, and the polymerization was initiated by placing the reaction vessel in a water bath heated to $50{ }^{\circ} \mathrm{C}$. After $24 \mathrm{~h}$, the polymer was removed from the vessel, lightly crushed, and washed with $\mathrm{MeOH} / 0.1 \mathrm{M} \mathrm{HCI}(60: 40 \mathrm{v} / \mathrm{v})$ for template removal. The non-imprinted polymer (NIP) was prepared in the same way but in the absence of template molecule. The resulting polymers were further crushed and sieved to isolate a grain size fraction of $25-50 \mu \mathrm{m}$. This fraction was used for all further tests.

Preparation of pHis-Imprinted Polymer Microspheres. Polymer microspheres (MIP3/NIP3) were prepared as reported previously ${ }^{32}$ by templated synthesis using AcNH@ $\mathrm{Si}(1.0 \mathrm{~g})$ as sacrificial template. In brief, the particles were firstly deaerated in a $50 \mathrm{~mL}$ Schlenk tube by three freeze-thaw cycles with intermittent $\mathrm{N}_{2}$-purging and then allowed to soak in prepolymerization mixtures, identical to those used to prepare MIP2 and NIP2, under continuous nitrogen flow. The volume prepolymerization mixture was adjusted to barely fill the internal pore system while avoiding particle aggregation. The mixtures were placed in a water bath and heated to $50{ }^{\circ} \mathrm{C}$ overnight. The resulting composite beads were treated in $3 \mathrm{M}$ $\mathrm{NH}_{4} \mathrm{HF}_{2}$ solution to remove the silica template, followed by pHis-template removal as described in the previous section.

Template Binding Tests. Each polymer $(10 \mathrm{mg})$ was suspended in $1.0 \mathrm{~mL}$ of an equimolar mixture of Fmoc-pTzaOEt, Fmoc-pTyr-OEt, and Fmoc-pSer-OEt (each $100 \mu \mathrm{M}$ ) prepared in $\mathrm{MeCN} / \mathrm{H}_{2} \mathrm{O}$ at different ratios buffered with $0.1 \%$ triethylamine (TEA). Each suspension was shaken vigorously for $2 \mathrm{~h}$ and then each sample was centrifuged. The supernatant $(500 \mu \mathrm{L})$ was dried by using a Genevac EZ-2 evaporator, then reconstituted in $40 \% \mathrm{MeCN}+0.1 \%$ trifluoroacetic acid (TFA) $(200 \mu \mathrm{L})$, and analyzed by reversed-phase high-performance liquid chromatography (HPLC). The column used was Prodigy $5 \mu \mathrm{m}$ ODS-3 $100 \AA$ (Phenomenex, $150 \times 4.6$ $\mathrm{mm}^{2}$ ). Mobile phases were (A) $99.9 \% \mathrm{H}_{2} \mathrm{O}+0.1 \%$ TFA and (B) $99.9 \% \mathrm{MeCN}+0.1 \%$ TFA. A linear gradient method involving $40 \% \mathrm{~B}$ to $60 \% \mathrm{~B}$ in $12 \mathrm{~min}$ at a flow rate of $0.6 \mathrm{~mL} /$ min was used. The injection volume was $50.0 \mu \mathrm{L}$ and the detection was performed by UV absorbance measurement at $265.0 \mathrm{~nm}$. All experiments were performed in three parallel replicates.

Synthesis of pHis Reference Peptides. MIP selectivity was probed using two different pHis peptides, $\mathrm{VpHI}$ and DRVYIpHPF, obtained by chemical phosphorylation of VHI and DRVYIHPF, respectively, using potassium phosphoramidate (see Supporting Information). ${ }^{25}$ This method is highly selective towards histidine and no other amino acid residues are phosphorylated. Furthermore, the major product of the reaction is the $\tau$-pHis isomer. The identity of the two pHis peptides was confirmed by electrospray ionization mass spectrometry (ESI MS). The chemical shifts of the imidazole ring protons in the ${ }^{1} \mathrm{H}$ nuclear magnetic resonance (NMR) spectra of VpHI peptide (Figure S2) were in agreement with previously reported data for $\tau$-pHis isomers. ${ }^{25,26}$ Amino acid sequencing by matrix-assisted laser desorption ionization tandem mass spectrometry (MALDI MS/MS) of the octapeptide (Figure S3) confirmed phosphorylation of the histidine residue.

Investigation of pHis Peptide Stability. A solution of each peptide $(50 \mu \mathrm{M})$ prepared in $0.1 \%$ TFA, $0.1 \% \mathrm{FA}$, and 10 $\mathrm{mM} \mathrm{NH}_{4} \mathrm{HCO}_{3}(\mathrm{pH}=8)$ was sampled at time points $0,1,2$, $4,6,12$, and $24 \mathrm{~h}$ followed by injection $(100 \mu \mathrm{L})$ and analysis by HPLC. The concentration of phosphorylated peptide was determined by using external standards.

Binding Test Using Tri-Peptide Standards. Each polymer $(10 \mathrm{mg})$ was suspended in $1.0 \mathrm{~mL}$ of an equimolar 
mixture of VpSI, VpHI, VEI, and VHI (each $20 \mu \mathrm{M}$ ) prepared in $\mathrm{MeCN} / \mathrm{H}_{2} \mathrm{O}$ at different ratios buffered with $0.1 \%$ TEA. After the binding test, the supernatant was dried and each sample was reconstituted using $10 \mathrm{mM} \mathrm{NH} \mathrm{NHCO}_{3}$ solution $(200 \mu \mathrm{L})$ and analyzed by reversed-phase HPLC. The column used was XBridge $\mathrm{C} 185 \mu \mathrm{m}\left(50 \times 4.6 \mathrm{~mm}^{2}\right)$. Mobile phases were (A) $10 \mathrm{mM} \mathrm{NH} \mathrm{HCO}_{3}$ and (B) $\mathrm{MeCN} / 10 \mathrm{mM}$ $\mathrm{NH}_{4} \mathrm{HCO}_{3}$ (9:1). A linear gradient method involving $0 \% \mathrm{~B}$ to $35 \% \mathrm{~B}$ in $18 \mathrm{~min}$ at a flow rate of $0.6 \mathrm{~mL} / \mathrm{min}$ was used. The injection volume was $50.0 \mu \mathrm{L}$ and the detection was performed by $\mathrm{UV}$ absorbance measurement at $265.0 \mathrm{~nm}$. All experiments were performed in three replicates.

$\boldsymbol{\beta}$-Elimination Protocol. VpSI and VpTI were dissolved separately in $0.1 \mathrm{M} \mathrm{Ba}(\mathrm{OH})_{2}(500 \mu \mathrm{L})$ in the presence of $\mathrm{VpHI}$ and $\mathrm{VHI}$ (concentration of each peptide $=5 \mathrm{mM}$ ). Each solution was kept at $50{ }^{\circ} \mathrm{C}$ overnight and the percentage dephosphorylation of $\mathrm{VPSI}$ and $\mathrm{VpTI}$ was analyzed at the time points $0,6,12,24$, and $48 \mathrm{~h}$ by HPLC. At each time point, the sample was firstly neutralized with $1.0 \mathrm{M} \mathrm{HCI}$ and acidified with $0.1 \% \mathrm{FA}$ to $\mathrm{pH}=5$. The sample was passed through a C18 silica gel column (activated with $\mathrm{MeCN}+0.1 \% \mathrm{FA}$ and equilibrated with $0.1 \%$ FA prior to use), the column was washed with $0.1 \% \mathrm{FA}$, and the peptides were eluted with $95 \%$ $\mathrm{MeCN}+0.1 \%$ TEA. Complete dephosphorylation of all phosphoesters was confirmed after $24 \mathrm{~h}$ of treatment with the base media.

Tryptic Digest of Bovine Serum Albumin (BSA). Bovine serum albumin (BSA, $10 \mathrm{mg}$ ) dissolved in $100 \mathrm{mM}$ $\mathrm{NH}_{4} \mathrm{HCO}_{3}(1 \mathrm{~mL})$ was reduced with $50 \mathrm{mM}$ dithiothreitol (DTT) for $30 \mathrm{~min}$ at $50{ }^{\circ} \mathrm{C}$ and alkylated with $100 \mathrm{mM}$ IAA for $30 \mathrm{~min}$ at room temperature in the dark. Then, the protein was digested with trypsin $(200 \mu \mathrm{g})$ for $24 \mathrm{~h}$ at $37^{\circ} \mathrm{C}$. The peptide sample was then acidified with TFA and desalted on a C18 silica gel column (Bond Elut C18, Analytichem International, CA). The column was first activated with $\mathrm{MeCN}+0.1 \%$ TFA $(2 \mathrm{~mL})$ and conditioned with $0.1 \%$ TFA $(2 \times 2 \mathrm{~mL})$. Finally, the peptides were eluted with $80 \% \mathrm{MeCN}$ $+0.1 \%$ TFA $(2 \mathrm{~mL})$; the digest was lyophilized and stored at $-20{ }^{\circ} \mathrm{C}$ prior to use.

pHis Peptide Enrichment by Combined MIP and $\beta$ Elimination. The mixture of $\mathrm{VpHI}(40 \mu \mathrm{M}), \mathrm{VpSI}(40 \mu \mathrm{M})$, and tryptic digest of BSA $(200 \mu \mathrm{M})$ (spiking level is 1:5) was prepared in $0.2 \mathrm{M} \mathrm{Ba}(\mathrm{OH})_{2}(25 \mu \mathrm{L})$ and equilibrated at $37^{\circ} \mathrm{C}$ for $2 \mathrm{~h}$. Then, $0.4 \mathrm{M}$ of $\left(\mathrm{NH}_{4}\right)_{2} \mathrm{SO}_{4}(12.5 \mu \mathrm{L})$ was added and $\mathrm{BaSO}_{4}$ was formed as a white precipitate. The sample was filtered to remove the insoluble $\mathrm{BaSO}_{4}$ salt and the residue was washed with water $(12.5 \mu \mathrm{L})$. The filtrate was diluted with $\mathrm{MeCN}+0.1 \%$ TEA $(950 \mu \mathrm{L})$. This sample $(200 \mu \mathrm{L})$ was mixed with MIP and NIP $(5 \mathrm{mg})$ separately in a $1.5 \mathrm{~mL}$ microcentrifuge plastic tube and shaken for $2 \mathrm{~h}$. After incubation, the samples were transferred to a $200 \mu \mathrm{L}$ pipette tip microcolumn protected with a C8 plug and passed through the microcolumn. The flow-through fraction was collected as a first step of the enrichment process. The washing step was performed with $95 \% \mathrm{MeCN}+0.1 \%$ TEA $(2 \times 200 \mu \mathrm{L})$ and the elution step was performed with $50 \% \mathrm{MeCN}+0.1 \%$ TEA $(2 \times 200 \mu \mathrm{L})$. Each fraction was dried (Genevac EZ-2 evaporator), redissolved in water $(200 \mu \mathrm{L})$, and then analyzed by HPLC with ESI-MS detection.

HPLC ESI-MS Analysis. The HPLC ESI-MS analyses were performed in the reversed-phase mode using an analytical C18 column (XBridgeTM C18 $5 \mu \mathrm{m} 50 \times 4.6 \mathrm{~mm}^{2}$, Waters, Milford, MA). The mobile phases were (A) $10 \mathrm{mM}$
$\mathrm{NH}_{4} \mathrm{HCO}_{3}$ and (B) $\mathrm{MeCN} / \mathrm{NH}_{4} \mathrm{HCO}_{3}$ (9:1). The chromatography was performed using a linear gradient from $0 \% \mathrm{~B}$ to $100 \% \mathrm{~B}$ in $30 \mathrm{~min}$ at a flow rate of $0.5 \mathrm{~mL} / \mathrm{min}$. The injection volume was $100 \mu \mathrm{L}$ and the detection was performed by UV absorbance measurement at $210 \mathrm{~nm}$ and ESI-MS (Waters, micromassZQ, MM1, LAA1108, Milford, MA) selective ion monitoring (SIM) with the mass window of 1.0 Da. The mass spectra were recorded in positive ion mode. The analysis parameters were set as follows: capillary voltage $5 \mathrm{kV}$, cone voltage $30 \mathrm{~V}$, source temperature $120{ }^{\circ} \mathrm{C}$, and desolvation temperature $250{ }^{\circ} \mathrm{C}$.

LC-MS/MS Analysis of Phosphopeptides. Dried peptide samples were redissolved and analyzed on a Dionex Ultimate 3000 RSLCnano system coupled online to an Orbitrap Fusion Tribrid mass spectrometer (Thermo Scientific). To avoid loss of pHis, dried fractions were redissolved in cold water, vortexed for $1 \mathrm{~min}$, and sonicated for 1 min immediately before LC-MS/MS analysis. The volume was adjusted so as to inject $60 \mathrm{fmol}$ of peptides in a single injection of $50 \mu \mathrm{L}$. The injected peptide samples were desalted on-line using a C18 trap column $(300 \mu \mathrm{m}$ i.d. $\times 5 \mathrm{~mm}, 5 \mu \mathrm{m}$, $100 \AA /$ PepMap TM 100, Thermo Scientific) for 3 min using $2 \% \mathrm{MeCN}$ in $0.1 \%$ FA. Peptides were eluted and separated on an in-house packed $75 \mu \mathrm{m}$ i.d. $\times 30 \mathrm{~cm}$ column with ReproSilPur $120 \mathrm{C} 181.9 \mu \mathrm{m}$ particles (Dr. Maisch) at a flow rate of $300 \mathrm{~nL} / \mathrm{min}$ with a linear gradient of $2-34 \%$ solvent B $(95 \%$ $\mathrm{MeCN}+0.1 \%$ FA) against solvent $\mathrm{A}\left(99.9 \% \mathrm{H}_{2} \mathrm{O}+0.1 \%\right.$ FA). ${ }^{27}$ Precursor ion (MS) spectra were acquired in the $\mathrm{m} / \mathrm{z}$ range $350-1800(2+$ to $4+$ ion charge state) at a mass resolution setting of $120 \mathrm{k}$ at $\mathrm{m} / z 400$ in profile mode, with an AGC target of $2 \mathrm{e} 5$ ions and $3 \mathrm{~s}$ cycle time. Precursor ions were fragmented with higher-energy collisional dissociation (HCD, NCE $=33 \%$ ) and dynamically excluded for $15 \mathrm{~s}$. Fragments were detected in the Orbitrap at a mass resolution setting of 15000 at $\mathrm{m} / z 400$ in centroid mode. For the analysis of the microspheres MIP3 and NIP3, MS1 mass resolution was set to $60 \mathrm{k}$ and precursors were fragmented with internally calibrated electron transfer dissociation (ETD) with HCD supplemental activation (EThcD). Fragment ions were analyzed in the Orbitrap at $30 \mathrm{k}$ mass resolution.

Enrichment of $\mathrm{VpHI}$ from Tripeptides Spiked in BSA $\boldsymbol{\beta}$-Casein Digest. The three short p-peptides (VpHI, VpSI, $\mathrm{VPTI}$ ) were combined in an equimolar mixture prior to mixing them with $\mathrm{BSA} / \beta$-Casein digest at $1: 10$ and $1: 20$ ratio, respectively. Fifty picomoles of BSA $/ \beta$-Casein were used per enrichment and were spiked with 5 pmol of each peptide for the $1: 10$ ratio and 2.5 pmol for the 1:20 ratio. Prior to solidphase extraction (SPE), the peptide mixture was mixed with $\mathrm{MeCN}$ and $0.1 \%$ TEA to a final solvent composition of $95 \%$ $\mathrm{MeCN}+0.1 \%$ TEA in $300 \mu \mathrm{L}$. Peptides were then incubated with $3 \mathrm{mg}$ of MIP or NIP for $2 \mathrm{~h}$ with vigorous shaking in a low-binding $1.5 \mathrm{~mL}$ Eppendorf tube. Three replicates for each spiking level were performed on each polymer, with three preenrichment control samples ( $R$, reference) for each spiking level. After the first incubation, tubes were centrifuged for 5 $\mathrm{min}$ at $14 \mathrm{k} \mathrm{rpm}$ before the liquid was collected. Next, the MIP/ NIP was washed with $400 \mu \mathrm{L}$ of load/wash solvent for $15 \mathrm{~min}$ and the liquid was pooled with the previous collection and labeled "loading + washing $(\mathrm{L}+\mathrm{W})$ " fraction. Phoshopeptides were recovered from the polymers by two sequential elutions with $500 \mu \mathrm{L}$ of elution (E) solvent $(99.9 \% \mathrm{MeOH}+0.1 \%$ TEA) for $15 \mathrm{~min}$ and then for $30 \mathrm{~min}$. The liquid fractions were pooled and labeled "E". Both $\mathrm{L}+\mathrm{W}$ and $\mathrm{E}$ fractions were 
dried by vacuum centrifugation and redissolved prior to LCMS/MS analysis. Every fraction was injected with the same theoretical amount of tripeptide $(60 \mathrm{fmol})$ by adjusting the volume. The peak areas of the tripeptides were calculated using the Genesis algorithm implemented in the LC-MS/MS data analysis software FreeStyle (v. 1.3, Thermo Scientific). Tryptic peptides from BSA $\beta$-Casein were identified and annotated by processing the LC-MS/MS data using the MASCOT search engine (Matrix Science) through Proteome Discoverer 2.4 (Thermo Scientific).

Chemical N-Phosphorylation of Myoglobin. Horse heart myoglobin $(\mathrm{Mb})$ was chosen for chemical $\mathrm{N}$-phosphorylation of histidines using potassium phosphoramidate (PPA). $\mathrm{Mb}$ contains 11 histidine residues and no reactive cysteine residues. Approximately $1 \mathrm{mg}$ of myoglobin was dissolved in 1 $\mathrm{mL}$ of water by thorough vortexing. PPA was added to the protein solution in a 1:600 protein/PPA molar ratio and incubated at room temperature for $24 \mathrm{~h}$. Phosphorylation was confirmed by intact protein mass determination using static nanoESI-MS (Orbitrap Fusion Tribrid, Thermo Scientific). Nanoelectrospray needles were obtained from Fisher Scientific (NC0355451). The protein (50 pmol) was desalted/ concentrated using a C 8 membrane plug in a $200 \mathrm{uL}$ pipette tip and eluted in a small volume of $50 \% \mathrm{MeCN}+1 \%$ FA. Mass spectra were acquired in the Orbitrap mass analyzer at $120 \mathrm{k}$ resolution with a $70 \% \mathrm{RF}$ lens, 5e5 AGC target, and $100 \mathrm{~ms}$ maximum injection time for a total acquisition time of $2 \mathrm{~min}$.

Enrichment of pHis Peptides from the Mixture of the $\mathrm{Mb} / \mathrm{BSA} / \boldsymbol{\beta}$-Casein Digest. Protein digests of equimolar mixtures of BSA, $\beta$-Casein, and histidine phosphorylated myoglobin were initially de-salted using porous $\mathrm{R} 3$ resin. A 200 $\mu \mathrm{L}$ pipette tip was plugged with $\mathrm{C} 18$ membrane and filled with $750 \mu \mathrm{g}$ of $\mathrm{R} 3$ resin. The beads were conditioned with $100 \%$ $\mathrm{MeCN}$ and equilibrated with $100 \mu \mathrm{L}$ of water. For either MIP or NIP, a total of $7.36 \mu \mathrm{g}$ of protein digest $(200 \mathrm{pmol})$ was loaded on the tip and the liquid passed through with manual centrifugation. The flow-through was collected and passed once more through the tip. The beads were washed twice with $100 \mu \mathrm{L}$ of water before being eluted with $50 \mu \mathrm{L}$ of $50 \% \mathrm{MeCN}$. The eluted peptides were dried and redissolved in water prior to SPE. The de-salted digest was re-dissolved in $60 \mu \mathrm{L}$ of $0.1 \%$ TEA and divided into three aliquots of $15 \mu \mathrm{L}(50 \mathrm{pmol})$. Then, the aliquots were diluted with $99.9 \% \mathrm{MeCN}+0.1 \%$ TEA to a final volume of $300 \mu \mathrm{L}$. LC-MS/MS analysis and peptide identification were performed as described in the previous section.

\section{RESULTS AND DISCUSSION}

Design and Synthesis of a First-Generation pHis MIP (MIP1). We previously showed that imprinted polymers (MIPs) prepared using urea-based N-3,5-bis(trifluoromethyl)-phenyl- $N^{\prime}$-4-vinylphenylurea, functional monomer 1 (Figure 1), display a high affinity for phosphorylated peptides ${ }^{28,29}$ and that the selectivity for either phosphoserine (pSer) or phosphotyrosine ( $\mathrm{pTyr}$ ) peptides can be programmed using the appropriate template. ${ }^{30-32}$ Mechanistically, the hydrogen bond-driven recognition and the charge-neutral resin character distinguish these phases from currently used phosphoenrichment tools, e.g., IMAC, $\mathrm{TiO}_{2}$, and antibodies, and could explain the reduced chargedependent sequence bias of the enriched phosphopeptide pool. Here we adopted the same urea-based approach for the recognition of histidine-phosphorylated peptides.
目 Fmoc-pTza-OEt $\square$ Fmoc-pSer-OEt 四 Fmoc-pTyr-OEt
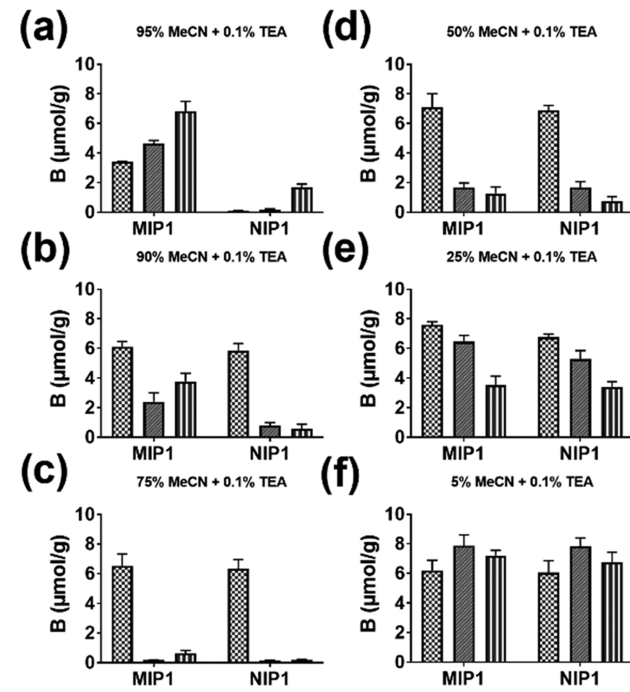

Figure 2. Absorbed amounts of Fmoc-pTza-OEt, Fmoc-pSer-OEt, and Fmoc-pTyr-OEt by imprinted and non-imprinted polymers in different solvent systems: (a) 95\%, (b) 90\%, (c) 75\%, (d) 50\%, (e) $25 \%$, and (f) $5 \% \mathrm{MeCN}$ and each buffered with $0.1 \%$ TEA.

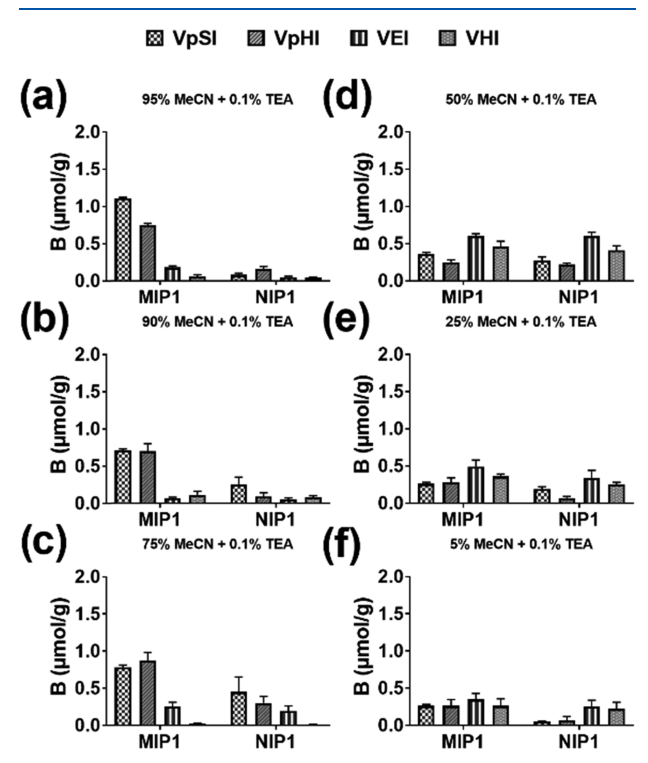

Figure 3. Amounts of VpSI, VpHI, VEI, and VHI bound to imprinted and non-imprinted polymers in different solvent systems: (a) $95 \%$, (b) $90 \%$, (c) $75 \%$, (d) $50 \%$, (e) $25 \%$, and (f) $5 \% \mathrm{MeCN}$ and each buffered with $0.1 \%$ TEA. 


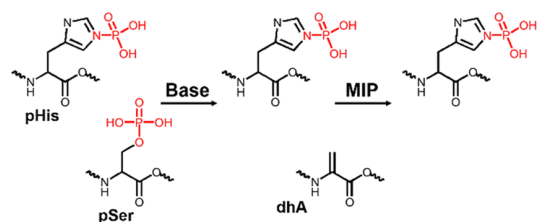

Figure 4. Concept of the MIP-based method for enrichment of $\mathrm{pHis}$ peptides. pSer (or pThr) undergoes facile dephosphorylation via $\beta$ elimination in the presence of a strong base (e.g., sodium or barium hydroxide) and it is converted to dehydroalanine ( $\mathrm{dhA})$. pHis stability is not affected under such conditions and it can be enriched by the MIP in the next step.
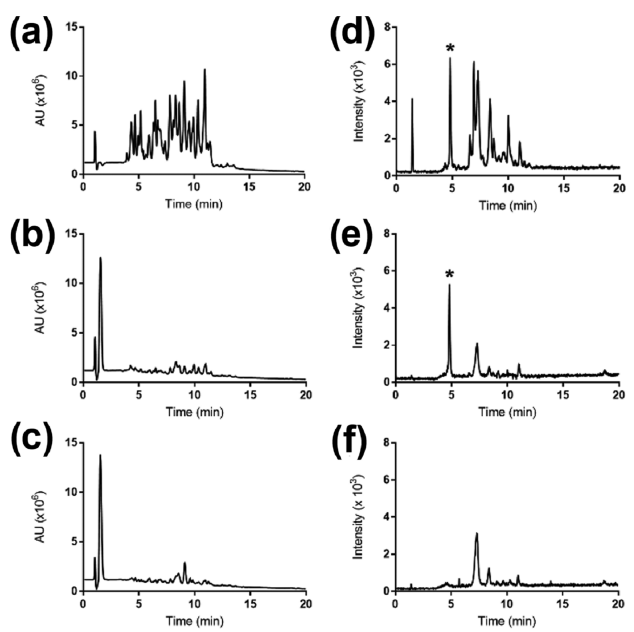

(e)
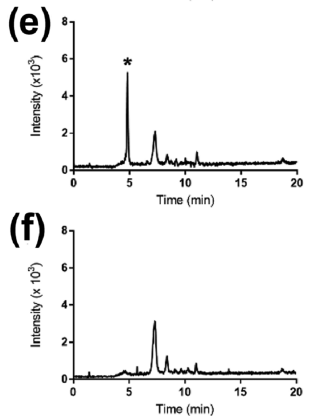

Figure 5. HPLC-UV chromatograms $(\mathrm{a}-\mathrm{c})$ and corresponding ESIMS selective ion monitoring (SIM, $m / z=447.5)$ spectra $(d-f)$ of the sample before enrichment (a, d) and elution fractions from MIP1 (b, e) and NIP1 (c, f). The VpHI peptide $(0.2 \mu \mathrm{mol})$ was spiked in BSA $(1 \mu \mathrm{mol})$ digest. The signal from $\mathrm{VpHI}$ is marked with an asterisk.

One of the key elements in the design of the imprinted polymer is the choice of the template. The labile nature of phosphohistidine posed many problems when used as an antigen to raise antibodies since it undergoes rapid dephosphorylation after injection into animals. ${ }^{10}$ Inspired by previous reports on stable phosphohistidine analogues, ${ }^{12,21,33}$ we prepared Fmoc-pTza-OEt (Figure 1), which mimics the $\tau$ $\mathrm{pHis}$ isomer, and used it as a template for imprinting. The template was synthesized according to Figure S1 using the copper-catalyzed click reaction. Imprinted polymers were then prepared by free radical polymerization using a 1:2 ratio of template (as its PMPH or TBA salt) to 1 , pentaerythritoltriacrylate (PETA) as cross-linker, and acetonitrile ( $\mathrm{MeCN})$ as porogen in bulk or microsphere format as described in the Supporting Information (Table S1).

Probing Affinity and Selectivity of Polymers in Simple Amino Acid and Peptide Mixtures. Previously reported protocols for MIP-based enrichment of pTyr and pSer peptides employed acidic (0.1\% TFA) loading, washing, and elution steps. ${ }^{28-30,34}$ Under these conditions the phosphate group is not fully protonated and with one oxygen negatively charged, it is a potent hydrogen bond acceptor that can interact with the urea $\mathrm{N}-\mathrm{H}$ hydrogen bond donor. Such conditions are however not appropriate for enrichment of $\mathrm{pHis}$ due to its acid-labile nature. Instead, phosphoramidates such as pHis are stable under basic conditions, and since the phosphate group is deprotonated, it engages in strong interactions with the urea functional groups of the MIP. We therefore used triethylamine (TEA) as mobile phase modifier to ensure the stability of $\mathrm{pHis}$ modification and to provide strong interactions with the imprinted polymer.

We first tested the affinity of MIP1 and NIP1 towards N,Cprotected amino acids pTza, pSer, and pTyr in $\mathrm{MeCN} / \mathrm{H}_{2} \mathrm{O}$ mixtures buffered with $0.1 \%$ TEA as basic modifier (Figure 2). As seen in Figure 2a, the contrasting retention behavior of MIP1 and NIP1 in 95\% MeCN reflects a strong impact of imprinting. Although the MIP captured each of the phosphorylated amino acids, the highest imprinting factor (IF $\left.=\mathrm{B}_{\mathrm{MIP}} / \mathrm{B}_{\mathrm{NIP}}\right)$ was registered for Fmoc-pTza-OEt (see Table S2). This reflects a slight memory for the template accompanied by a rather strong nonspecific contribution. The latter increased dramatically with further increase in the water content. Thus, retention differences between MIP1 and NIP1 could no longer be observed at $10 \%$ water and beyond. However, under these conditions both polymers showed a pronounced selectivity for the pTza template. Given that both MIP1 and NIP1 displayed this effect, we attribute this to an involvement of the triazole ring in the polymer template interactions. A plausible hydrogen-bonding motif places the triazole nitrogen juxtaposed to the phosphate group at hydrogen bond distance from the urea group (Figure S4), thereby stabilizing additionally the monomer-template complexes.

We next probed the ability of the imprinted polymer to recognize the histidine phosphorylated peptide $\mathrm{VpHI}$ in the presence of structurally related VHI, VpSI, and VEI in $\mathrm{MeCN} /$ $\mathrm{H}_{2} \mathrm{O}$ mixtures $+0.1 \%$ TEA as modifier (Figure 3). Also, in this case we observed the strongest imprinting effect in $95 \%$ $\mathrm{MeCN}$, reflected in a high binding on the MIP1 and low binding on the NIP1 (see IF values, Table S3). Moreover, despite a clear preference for the phosphopeptides VpSI and $\mathrm{VpHI}$ over the negatively charged VEI, no significant pHis selectivity was noted in this test. Instead, we noted an overall decrease in both binding and imprinting effect with increasing water content. The absence of pHis selectivity further supports the above-invoked role of the triazole nitrogen in the urea template interactions (vide supra).

Considering the lack of strong pHis discrimination, we turned to an alternative enrichment approach where pSer and pThr peptides were selectively dephosphorylated in the presence of $\mathrm{pHis}$ peptides followed by the MIP-based enrichment step (Figure 4).

pHis Discrimination by $\beta$-Elimination of pSer and pThr. Phosphohistidine modification is known to undergo facile dephosphorylation in acidic environment. The synthesized VpHI and DRVYIpHPF were therefore subjected to a stability test (Figure S5). Indeed, the studied peptides were dephosphorylated in the presence of acids in contrast to basic buffer, where no significant decomposition was observed even after $24 \mathrm{~h}$. However, a short treatment $(<30 \mathrm{~min})$ of the peptides with acids did not lead to extensive dephosphorylation. Using short acidic LC gradients is another approach for detection of pHis by LC-MS/MS. ${ }^{14,19}$ In another study, 

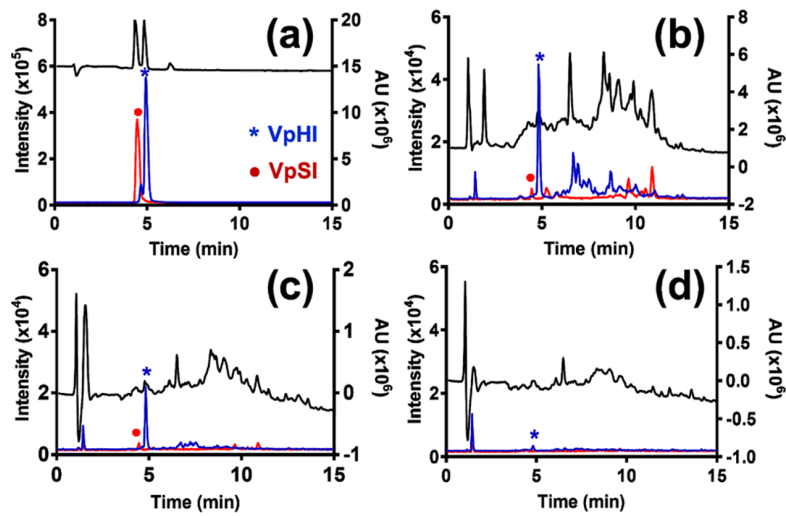

- m/z $447.5-m / 2397.4-U V @ 210 n m$

Figure 6. HPLC-UV chromatogram and corresponding ESI-MS selective ion monitoring of VpHI $(\mathrm{m} / z=447.5)$ and VpSI $(\mathrm{m} / z=397.4)(\mathrm{a})$, spiked in BSA tryptic digest and treated with $\mathrm{Ba}(\mathrm{OH})_{2}(\mathrm{~b})$, and in the elution fractions from MIP1 (c) and NIP1 (d).
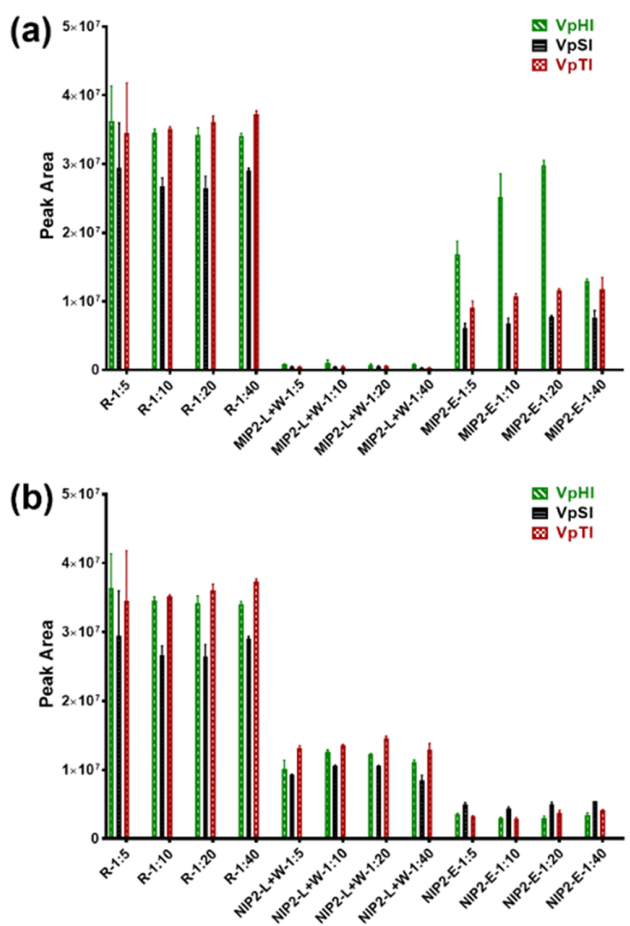

Figure 7. Peak areas of the three phosphorylated tripeptides obtained from each SPE fractions obtained in MIP2 (a) and NIP2 (b) at 1:5, $1: 10,1: 20$, and $1: 40$ spiking levels $(\mathrm{R}=$ reference, $\mathrm{L}+\mathrm{W}=$ loading + washing, $\mathrm{E}=$ pooled elution).

copper-immobilized metal ion affinity chromatography $\left(\mathrm{Cu}^{2+}\right.$ IMAC) was adopted for extraction of pHis peptide from a
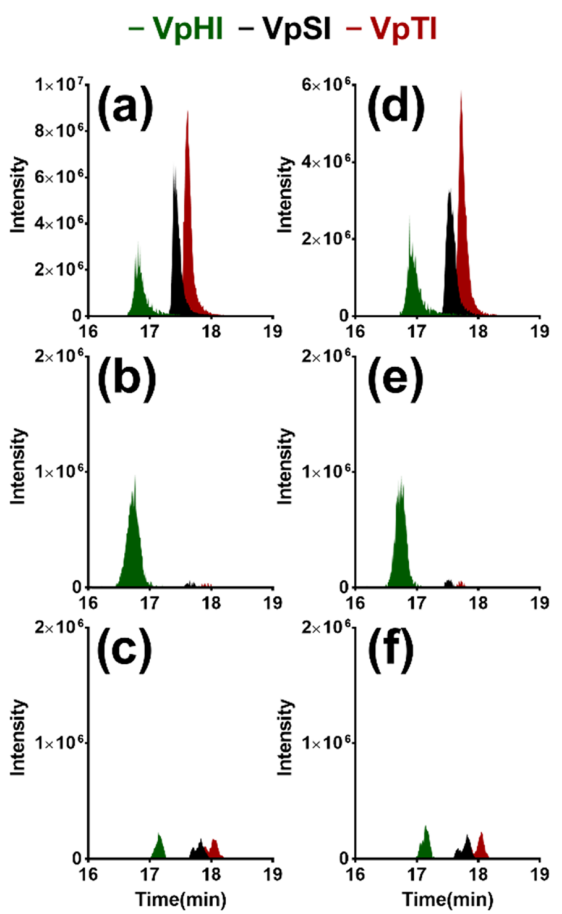

Figure 8. Extracted chromatogram of tripeptides (VpHI (green), VpSI (black), and VpTI (red)) for 1:10 $(\mathrm{a}-\mathrm{c})$ and 1:20 (d-f) spiking level from pre-enrichment (a,d), elution fractions obtained from MIP2 (b, e), and those obtained from $\operatorname{NIP2}(c, f)$.

digest of phosphorylated HPr protein from Escherichia coli. ${ }^{16}$ The sample was in this case loaded on a column conditioned with $0.1 \%$ acetic acid and the washing step was performed with 

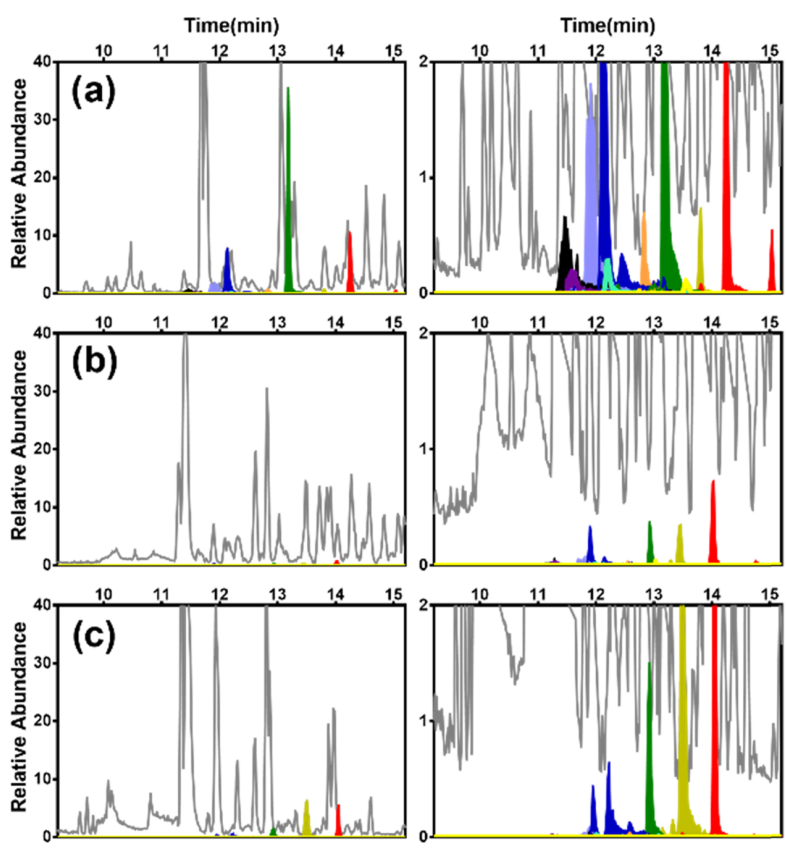

- Full

- [p]HPGDFGADAQGAMTK

- GH[p]HEAELKPLAQS[p]HATK

- VEADIAG[p]HGQEVLIR

- LFTH[P]HPETLEK

- [p]HPGDFGADAQGA[ox]MTK

- G[p]H[p]HEAELKPLAQS[p]HATK

- KGH[p]HEAELKPLAQS[p]HATK

- GH[p]HEAELKPLAQSHATK

- KG[p]H[p]HEAELKPLAQS[p]HATK

- [p]HPGDFGADA[de]QGAMTK

Figure 9. Extracted chromatograms highlighting histidine-phosphorylated peptides (indicated in color) corresponding to the elution fractions after enrichment using (a) MIP3 and (b) NIP3, and (c) no enrichment of a 1:1:1 BSA/ $\beta$-Casein/myoglobin digest (the range for relative abundance is from 0 to 40 on the left and from 0 to 2 in the right).

$0.1 \%$ acetic acid in $50 \% \mathrm{MeCN}$. However, the stability of individual $\mathrm{pHis}$ peptides can vary depending on the sequence and thus ideally any acidic treatment should be avoided.

We therefore took advantage of the contrasting stability profile of $\mathrm{pHis}$ and $\mathrm{pSer} / \mathrm{pThr}$ in alkaline environment (Figure 4). This approach was used to distinguish these modifications in a histidine kinase assay. ${ }^{35,36}$ Both pSer and $\mathrm{pThr}$ undergo facile dephosphorylation via $\beta$-elimination in a strongly alkaline environment. This reaction combined with subsequent Michael addition was used as a chemical derivatization method in protocols for enrichment of pSer and pThr peptides. ${ }^{37,38}$ Treatment of the mixture of $\mathrm{VpSI}$ and $\mathrm{VpHI}$ with $1 \mathrm{M} \mathrm{NaOH}$ at $60{ }^{\circ} \mathrm{C}$ for $30 \mathrm{~min}$ led to complete dephosphorylation of $\mathrm{VpSI}$ to give VdhAI (dhA = dehydroalanine). VpHI was not affected under these conditions. The sample was used in a binding equilibrium test (see Supporting Information for experimental). It was clear that VdhAI did not bind to the MIP1 and thus only VpHI was captured by the imprinted polymer (Figure S6). This result verifies the advantage of $\beta$ elimination for specific capture of phosphoamidates.

Probing Affinity and Selectivity with Spiked BSA Digest. After we confirmed the affinity of the MIP1 for the histidine phosphorylated peptide in a simple model system, we turned to more complex samples. As an initial test, we spiked $200 \mathrm{pmol}$ of $\mathrm{VpHI}$ peptide in $1 \mathrm{nmol}$ of BSA tryptic digest and incubated this mixture with MIP1 followed by transfer of the suspension to a micro-column. Next, washing and elution steps were performed as described in the Experimental Section.

The original sample, the combined flow-through and washing fractions, and elution fractions from MIP1 and NIP1 were analyzed by LC ESI MS with selective ion monitoring (SIM) set at $m / z 447.5$ corresponding to the mass of $\mathrm{VpHI}$ peptide (Figures 5 and S7). Treatment of the sample with both MIP1 and NIP1 led to a significant sample cleanup as can be seen from the UV chromatograms of the elution fractions (Figure $5 b, c$ ).

However, the pHis peptide was found only in the elution fraction from the MIP1 (Figure 5e). The NIP1 on the other hand did not retain this peptide, as it was present only in the combined flow-through and washing fraction (Figure S7d).

Repeating the test using DRVYIpHPF as pHis model peptide gave a similar result and thus a significant sample cleanup (Figure S8). These results confirmed the ability of the MIP1 to recognize $\mathrm{pHis}$ peptides in a protein digest. 
Probing Affinity and Selectivity with Base Treated Tryptic Peptides. The dominating phosphorylated residues in proteins are pSer and pThr. The high abundance of these motifs can mask less abundant motifs such as pTyr and especially labile motifs such as pHis. Focusing on the latter, base-stable phosphorylation concept, we developed a protocol where the affinity for $\mathrm{pHis}$ was enhanced by elimination of the interference of the most abundant base-labile phosphorylations (pSer and $\mathrm{pThr}$ ).

Although sodium hydroxide has been frequently used in the past for $\beta$-elimination of $\mathrm{p}$-peptides, the inherent disadvantage of this approach is the sample desalting step, which requires chromatographic purification and thus generates a risk of sample loss. ${ }^{39-41}$ To minimize sample handling, we chose barium hydroxide as the alkaline agent. $\mathrm{Ba}(\mathrm{OH})_{2}$ can be removed from the sample by precipitation in a form of waterinsoluble barium sulfate and simple filtration. ${ }^{42}$ Furthermore, it is more efficient in $\beta$-elimination and can be used at lower concentration (0.1-0.2 M compared to $1 \mathrm{M}$ for $\mathrm{NaOH}$ ), thus minimizing the degradation of peptides (Figure S9). Both VPSI and VpTI peptides dephosphorylated after treatment with $0.1 \mathrm{M} \mathrm{Ba}(\mathrm{OH})_{2}$ in $24 \mathrm{~h}$. The samples were further tested with MIP1 and NIP1. It was shown that the dephosphorylated forms of pSer and pThr tri-peptides, VdhAI and VmdhAI (mdhA, $\beta$-methyldehydroalanine), respectively, did not bind to the MIP and only VpHI was captured with enhanced binding percentage (Figure S9c). This result confirms that combining the $\beta$-elimination of phosphoesters (pSer and $\mathrm{pThr}$ ) with MIPbased enrichment enhances the specific capture of phosphoamidates (pHis).

As a proof of concept we used a sample of tryptically digested BSA spiked with both $\mathrm{VpSI}$ and $\mathrm{VpHI}$ at 1:5 spiking level. The sample was treated with barium hydroxide in order to deplete the pSer peptide.

The samples both before and after treatment with MIP1 and NIP1 were analyzed by LC-MS (Figures 6 and S10) using SIM of ions with $m / z=447.5$ (VpHI) and $m / z=397.4$ (VpSI). Compared to the reference sample with only VpSI and $\mathrm{VpHI}$ (Figure 6a), it was clear that treatment of the spiked sample with $\mathrm{Ba}(\mathrm{OH})_{2}$ resulted in dephosphorylation of $\mathrm{VpSI}$ (Figure 6b). This sample was then treated with MIP1 or NIP1; only the MIP1 extraction resulted in significant enrichment of the pHis peptide (Figure $6 \mathrm{c}, \mathrm{d}$ ). The HPLC-UV chromatograms confirmed that a significant cleanup had been achieved.

Unfortunately, testing this method at lower spiking levels and with more complex digests led to low recoveries and reduced selectivity (Figure S11). We therefore turned to a different strategy for further optimization of the pHis-MIP affinity and selectivity.

Design and Synthesis of a Second Generation of pHis-MIP (MIP2). Comprehensive optimization of imprinted polymer performance involves both structural and compositional parameters. In case of the hydrogen bond-mediated imprinting of phosphoanions using urea monomers, the binding affinity and selectivity are influenced by both monomer and template structural parameters. This was covered in depth in our previous report. ${ }^{43}$ The nature of the counter-cation has been shown to strongly influence the template-monomer complexation and in turn the target affinity exerted by the MIP. Enhanced complex stability is obtained by the use of quaternary ammonium ions like tetrabutylammonium (TBA), also accompanied by pronounced counterion memory effects. ${ }^{44}$
Focusing on this parameter, we prepared MIP2/NIP2 (Table S1) in a similar manner to MIP1/NIP1 but by replacing the tertiary amine PMP with the quaternary ammonium base TBA.OH. The polymers were freed from template and processed in an otherwise identical manner. The polymers were compared by testing their ability to enrich the model $\mathrm{pHis}$ peptide target $(\mathrm{VpHI})$ from structurally similar $\mathrm{VPSI}$ and VpTI tripeptides at four spiking levels of BSA $/ \beta$ Casein digests (Figure S12a).

We reasoned that pHis selectivity in this case would provide unequivocal evidence for the presence of side-chain-discriminative binding sites. With most of the spiked peptides recovered in the load/wash fractions, both NIPs failed to enrich any of the targets regardless of spiking level (Figures S13, S14, and $7 \mathrm{~b}$ ). MIP1 prepared using tertiary amine counterion $\left(\mathrm{PMPH}^{+}\right)$revealed a similar behavior (Figure $\mathrm{S} 13 \mathrm{a}$ ). On the other hand, MIP2 increased VpHI abundance relative to the other two tripeptides (Figure $7 \mathrm{a}$ ) especially at spiking ratios 1:10 and 1:20, whereas the effect was lost at 1:40. The possible reasons for this behavior are discussed below. To further confirm this observation, the experiment was repeated in triplicate at spiking levels 1:10 and 1:20 (Figure S14).

Here, MIP2 displayed pronounced target selectivity as seen by the relative peak area ratio (ApHis/(ApSer + ApThr) exceeding 10 of the spiked peptides in the eluted fractions (Figure 8). The results showed that the choice of counterion, in addition to the template and urea host monomer, plays a crucial role in the generation of high-affinity sites compatible with complex matrices.

LC-MS Test of pHis Peptide Enrichment Using MIP2. To test whether MIP2 and NIP2 were suitable for enrichment of tryptic pHis peptides from a more complex protein sample, we generated a tryptic digest of histidine phosphorylated myoglobin, BSA, and $\beta$-Casein. The enrichment protocol is shown in Figure S12b and detailed in the Experimental Section. Despite the promising results observed for the model tripeptides, the results for the endogenous tryptic peptides were not as expected, as judged by the number of phosphopeptides identified (Figure S15) and pHis peptide abundance (Figure S16). These results may have several causes. We previously ascribed the relatively low phosphoenrichment observed for complex digests to the hydrophobic character of the MIP scaffold resulting in rapid fouling and blockage of access to the discriminative sites.

Moreover, the polymers in this study were amorphous resins with a wide distribution of pores ranging from micro-pores with limited peptide access to accessible meso- or macro-pores. This may result in a size-dependent binding preference and poor accessibility for larger-sized peptides.

Enrichment of Tryptic pHis Peptides Using Controlled-Pore-Size-Imprinted Polymers (MIP3). We previously introduced hierarchically phosphopeptide epitopeimprinted polymers in a microsphere format to improve the overall binding efficiency and minimize the biomolecule size bias. $^{32}$ The resulting mesoporous microspheres (MIP3) featured a controlled and narrow pore size distribution and were capable of enriching larger p-peptide fragments.

To test the approach, we prepared $\mathrm{pHis}$ microspheres MIP3 and NIP3 (Table S1) and tested them on the His phosphorylated myoglobin, BSA, and $\beta$-Casein digest according to the protocol in Figure S12b. Figures 9 and S17 show the extracted chromatograms of the pre-enrichment control 
sample and post-enrichment (elution) sample using the two mesoporous capture materials with pHis peptides indicated in color. The latter comprise 10 mono- and multiple-Hisphosphorylated peptides ranging from 11 to 18 amino acids in length (Table S4).

First, we noted a striking reduction of the matrix-related peaks (shown in gray) after MIP enrichment, most notably for retention times exceeding $15 \mathrm{~min}$ (Figure S17). All modified peptides were identified in the MIP3 eluate (Figure 9a and Table S4), whereas the NIP3 revealed only modified peptides of low abundance (Figure 9b). Equally striking was the ability of the MIP3 to capture multiply phosphorylated peptides (light blue, orange, purple, and turquoise chromatograms). Confident phosphorylation site localization was achieved by tandem mass spectrometry using EThcD fragmentation, producing high amino acid sequence coverage and conserving the PTM on fragment ions (Figure S18).

The number of peptide spectrum matches (PSMs) of pHis peptides (Table S4 and Figure S19) confirmed the high selectivity of MIP3 for pHis-containing peptides. We observed a strong increase in the number of $\mathrm{pHis}$ peptide PSMs relative to other phosphopeptides (pSer, pThr), also when compared to the pre-enrichment control sample and the eluate of NIP3. We therefore conclude that a dual MIP templating strategy and microsphere format can be used to achieve enrichment of phosphohistidine-containing peptides obtained by tryptic digestion of phosphoproteins.

\section{CONCLUSIONS}

Protein histidine phosphorylation was reported more than 50 years ago but its biological role is still poorly understood. For many years, the acid-labile nature of pHis hampered development of methods for detection and analysis of this important PTM. This is now changing as new bioanalytical approaches and efficient tools are emerging. The molecularly imprinted polymer is such a tool that enables enrichment of $\mathrm{pHis}$ peptides under mild $\mathrm{pH}$ conditions. Using a refined $\mathrm{pHis}$ selective capture phase with tailored porosity and binding chemistry we have demonstrated a method, MIP3, offering the possibility to extract pHis peptides from complex mixtures under such mild conditions. This now opens new possibilities for highly robust modification-specific peptide enrichment strategies that we will continue to explore. We foresee that MIP-based methods for enrichment of pHis will play an important role in unraveling the biological roles of phosphohistidine in proteins using large-scale proteomics approaches.

\section{- ASSOCIATED CONTENT}

\section{(s) Supporting Information}

The Supporting Information is available free of charge at https://pubs.acs.org/doi/10.1021/acs.analchem.0c04474.

Detailed overview of experimental section outlining synthesis of template, reference peptide, and supporting tables and graphs (PDF)

\section{AUTHOR INFORMATION}

\section{Corresponding Author}

Börje Sellergren - Department of Biomedical Science, Faculty of Health and Society, Malmö University, 20506 Malmö, Sweden; $\odot$ orcid.org/0000-0002-2392-3305;

Email: borje.sellergren@mau.se

\section{Authors}

Anll Incel - Department of Biomedical Science, Faculty of Health and Society, Malmö University, 20506 Malmö, Sweden; $\odot$ orcid.org/0000-0003-1723-9803

Ignacio Arribas Díez - Department of Biochemistry \& Molecular Biology and VILLUM Center for Bioanalytical Sciences, University of Southern Denmark, DK-5230 Odense M, Denmark; ๑ orcid.org/0000-0001-7064-5283

Celina Wierzbicka - Department of Biomedical Science, Faculty of Health and Society, Malmö University, 20506 Malmö, Sweden

Katarzyna Gajoch - Department of Biomedical Science, Faculty of Health and Society, Malmö University, 20506 Malmö, Sweden

Ole N. Jensen - Department of Biochemistry \& Molecular Biology and VILLUM Center for Bioanalytical Sciences, University of Southern Denmark, DK-5230 Odense M, Denmark; $\odot$ orcid.org/0000-0003-1862-8528

Complete contact information is available at:

https://pubs.acs.org/10.1021/acs.analchem.0c04474

\section{Author Contributions}

C.W. performed the initial experiments with assistance from K.G. C.W. wrote the first version of the manuscript with contributions from B.S. and O.N.J. A.I. worked on the new recipe for MIP synthesis and collaborated with I.A.D. on the quantification by mass spectrometry of $\mathrm{pHis}$ peptides at lower spiking levels. I.A.D. performed all LC-MS/MS experiments and data analysis, and synthesized pHis myoglobin samples under the supervision of O.N.J. B.S. and O.N.J. initiated and supervised the project. All authors contributed to the writing of this manuscript and approved the final version of the manuscript.

\section{Notes}

The authors declare no competing financial interest.

\section{ACKNOWLEDGMENTS}

This work was supported by the EU-funded Marie Curie ITN project PEPMIP (PITN-GA-2010-264699) and the Marie Skłodowska-Curie Actions (H2020-MSCA-ITN-2016, 722171-Biocapture). Proteomics and mass spectrometry research at SDU is supported by generous grants from the VILLUM Foundation to the VILLUM Center for Bioanalytical Sciences (Grant No. 7292 to O.N.J.) and from the Danish Ministry of Higher Education and Science to the research infrastructure PRO-MS: Danish National Mass Spectrometry Platform for Functional Proteomics (Grant No. 5072-00007B to O.N.J.).

\section{REFERENCES}

(1) Cohen, P. Nat. Rev. Drug Discovery 2002, 1, 309-315.

(2) Li, D.; Agrellos, O. A.; Calderone, R. Curr. Opin. Microbiol. 2010, 13, 424-430.

(3) Khorchid, A.; Ikura, M. Int. J. Biochem. Cell Biol. 2006, 38, 307312 .

(4) Hérivaux, A.; So, Y. S.; Gastebois, A.; Latgé, J. P.; Bouchara, J. P.; Bahn, Y. S.; Papon, N. PLoS Pathog. 2016, 12, No. e1005683.

(5) Besant, P. G.; Tan, E.; Attwood, P. V. Int. J. Biochem. Cell Biol. 2003, 35, 297-309.

(6) Tan, E.; Besant, P. G.; Zu, X. L.; Turck, C. W.; Bogoyevitch, M. A.; Lim, S. G.; Attwood, P. V.; Yeoh, G. C. Carcinogenesis 2004, 25, 2083-2088.

(7) Attwood, P. V. Biochem. Soc. Trans. 2013, 41, 1023-1028. 
(8) Kee, J. M.; Muir, T. W. ACS Chem. Biol. 2012, 7, 44-51.

(9) Matthews, H. R. Pharmacol. Ther. 1995, 67, 323-350.

(10) Attwood, P. V.; Piggott, M. J.; Zu, X. L.; Besant, P. G. Amino Acids 2007, 32, 145-156.

(11) Fuhs, S. R.; Hunter, T. Curr. Opin. Cell Biol. 2017, 45, 8-16.

(12) Mukai, S.; Flematti, G. R.; Byrne, L. T.; Besant, P. G.; Attwood,

P. V.; Piggott, M. J. Amino Acids 2012, 43, 857-874.

(13) Besant, P. G.; Attwood, P. V. Mol. Cell. Biochem. 2009, 329, 93-106.

(14) Kleinnijenhuis, A. J.; Kjeldsen, F.; Kallipolitis, B.; Haselmann, K. F.; Jensen, O. N. Anal. Chem. 2007, 79, 7450-7456.

(15) Collins, M. O.; Yu, L.; Choudhary, J. S. Proteomics 2007, 7, 2751-2768

(16) Napper, S.; Kindrachuk, J.; Olson, D. J. H.; Ambrose, S. J.; Dereniwsky, C.; Ross, A. R. S. Anal. Chem. 2003, 75, 1741-1747.

(17) Ruprecht, B.; Koch, H.; Domasinska, P.; Frejno, M.; Kuster, B.; Lemeer, S. Methods Mol. Biol. 2017, 1550, 47-60.

(18) Ruprecht, B.; Koch, H.; Medard, G.; Mundt, M.; Kuster, B.; Lemeer, S. Mol. Cell. Proteomics 2015, 14, 205-215.

(19) Potel, C. M.; Lin, M. H.; Prust, N.; Van Den Toorn, H. W. P.; Heck, A. J. R.; Lemeer, S. Anal. Chem. 2019, 91, 5542-5547.

(20) Potel, C. M.; Lin, M. H.; Heck, A. J. R.; Lemeer, S. Nat. Methods 2018, 15, 187-190.

(21) Kee, J. M.; Villani, B.; Carpenter, L. R.; Muir, T. W. J. Am. Chem. Soc. 2010, 132, 14327-14329.

(22) Kee, J. M.; Oslund, R. C.; Perlman, D. H.; Muir, T. W. Nat. Chem. Biol. 2013, 9, 416-421.

(23) Kee, J. M.; Oslund, R. C.; Couvillon, A. D.; Muir, T. W. Org. Lett. 2015, 17, 187-189.

(24) Fuhs, S. R.; Meisenhelder, J.; Aslanian, A.; Ma, L.; Zagorska, A.; Stankova, M.; Binnie, A.; Al-Obeidi, F.; Mauger, J.; Lemke, G.; Yates, J. R.; Hunter, T. Cell 2015, 162, 198-210.

(25) Medzihradszky, K. F.; Phillipps, N. J.; Senderowicz, L.; Wang, P.; Turck, C. W. Protein Sci. 1997, 6, 1405-1411.

(26) Schrecker, O.; Stein, R.; Hengstenberg, W.; Gassner, M.; Stehlik, D. FEBS Lett. 1975, 51, 309-312.

(27) Kovalchuk, S. I.; Jensen, O. N.; Rogowska-Wrzesinska, A. Mol. Cell. Proteomics 2019, 18, 383-390.

(28) Emgenbroich, M.; Borrelli, C.; Shinde, S.; Lazraq, I.; Vilela, F.; Hall, A. J.; Oxelbark, J.; De Lorenzi, E.; Courtois, J.; Simanova, A.; Verhage, J.; Irgum, K.; Karim, K.; Sellergren, B. Chem. - Eur. J. 2008, 14, 9516-9529.

(29) Helling, S.; Shinde, S.; Brosseron, F.; Schnabel, A.; Müller, T.; Meyer, H. E.; Marcus, K.; Sellergren, B. Anal. Chem. 2011, 83, 18621865 .

(30) Chen, J.; Shinde, S.; Subedi, P.; Wierzbicka, C.; Sellergren, B.; Helling, S.; Marcus, K. J. Chromatogr. A 2016, 1471, 45-50.

(31) Bllaci, L.; Torsetnes, S. B.; Wierzbicka, C.; Shinde, S.; Sellergren, B.; Rogowska-Wrzesinska, A.; Jensen, O. N. Anal. Chem. 2017, 89, 11332-11340.

(32) Wierzbicka, C.; Torsetnes, S. B.; Jensen, O. N.; Shinde, S.; Sellergren, B. RSC Adv. 2017, 7, 17154-17163.

(33) McAllister, T. E.; Nix, M. G.; Webb, M. E. Chem. Commun. 2011, 47, 1297-1299.

(34) Chen, J.; Shinde, S.; Koch, M. H.; Eisenacher, M.; Galozzi, S.; Lerari, T.; Barkovits, K.; Subedi, P.; Krüger, R.; Kuhlmann, K.; Sellergren, B.; Helling, S.; Marcus, K. Sci. Rep. 2015, 5, No. 11438.

(35) Wei, Y. F.; Matthews, H. R. Methods Enzymol. 1991, 200, 388414

(36) Tan, E.; Zu, X. L.; Yeoh, G. C.; Besant, P. G.; Attwood, P. V. Anal. Biochem. 2003, 323, 122-126.

(37) Oda, Y.; Nagasu, T.; Chait, B. T. Nat. Biotechnol. 2001, 19, $379-382$.

(38) McLachlin, D. T.; Chait, B. T. Anal. Chem. 2003, 75, 68266836.

(39) Goshe, M. B.; Conrads, T. P.; Panisko, E. A.; Angell, N. H.; Veenstra, T. D.; Smith, R. D. Anal. Chem. 2001, 73, 2578-2586.

(40) Li, W.; Boykins, R. A.; Backlund, P. S.; Wang, G.; Chen, H. C. Anal. Chem. 2002, 74, 5701-5710.
(41) Steen, H.; Mann, M. J. Am. Soc. Mass Spectrom. 2002, 13, 9961003.

(42) Thompson, A. J.; Hart, S. R.; Franz, C.; Barnouin, K.; Ridley, A.; Cramer, R. Anal. Chem. 2003, 75, 3232-3243.

(43) Shinde, S.; Incel, A.; Mansour, M.; Olsson, G. D.; Nicholls, I A.; Esen, C.; Urraca, J.; Sellergren, B. J. Am. Chem. Soc. 2020, 142, 11404-11416.

(44) Wagner, S.; Zapata, C.; Wan, W.; Gawlitza, K.; Weber, M.; Rurack, K. Langmuir 2018, 34, 6963-6975. 


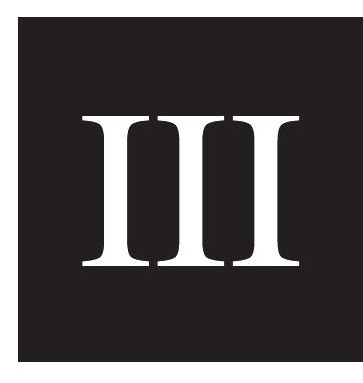




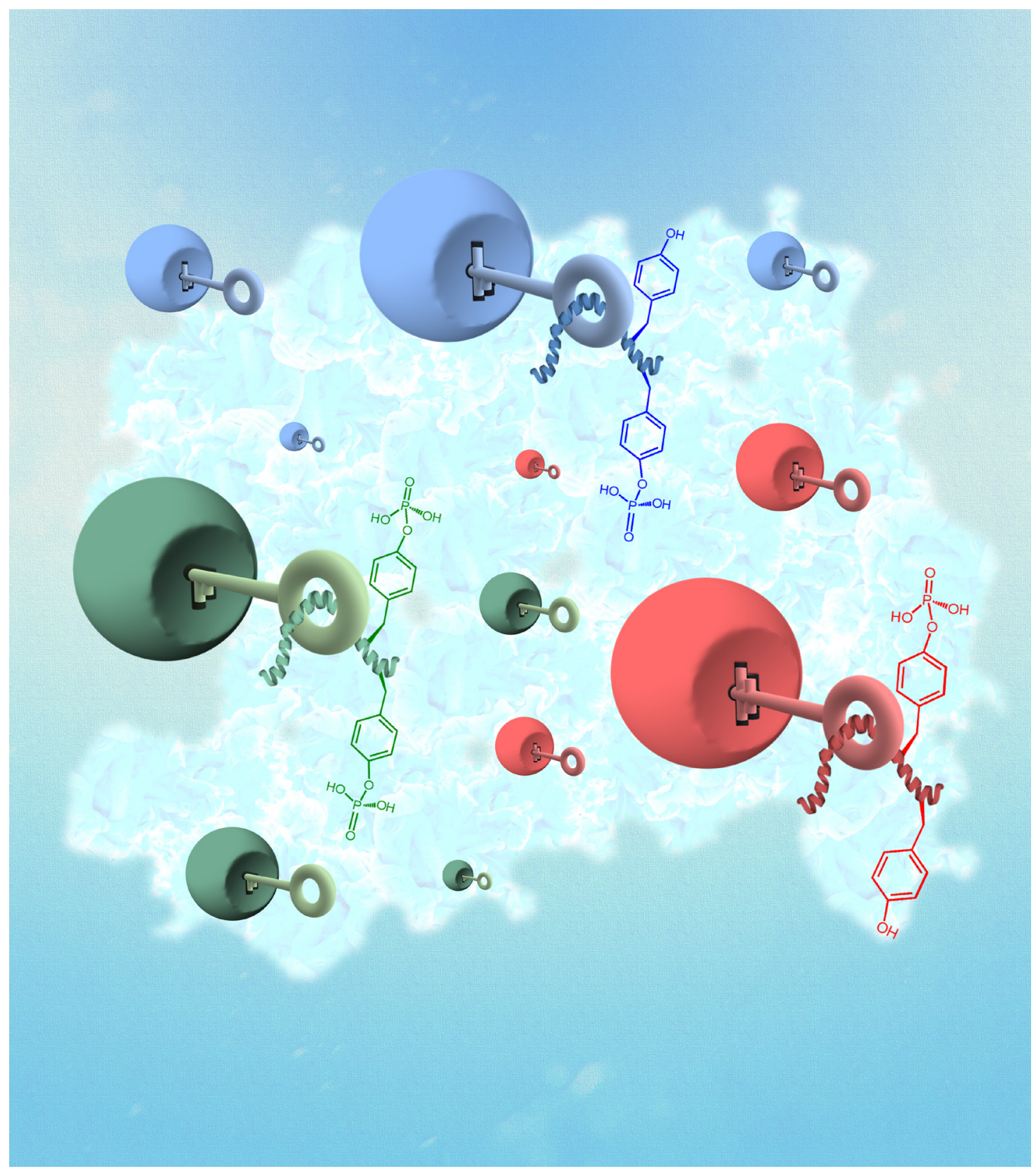




\title{
MIP-Binders for Sequence Specific
}

\section{Phosphopeptide Capture of ZAP-70 regulatory motifs}

\author{
Anıl Incel ${ }^{1}$, Sudhirkumar Shinde ${ }^{1}$, Ignacio A. Diez ${ }^{2}$, Alper Yılmaz ${ }^{1}$, Thomas Sjoberg ${ }^{1}$, Maria M. \\ Stollenwerk $^{1}$, Ole N. Jensen ${ }^{2}$ and Börje Sellergren ${ }^{1}$
}

\begin{abstract}
Immunoaffinity enrichment based on antipeptide antibodies coupled to mass spectrometry based identification and quantification is considered a promising approach to translate proteomics to clinical assays with a diagnostic value. Unfortunately, there is currently a lack of adequate tools for studying phospho-signaling networks, which hampers the development of effective diagnostics and treatment. This includes phosphopeptide specific antibodies which are either scarce or lack adequate performance. We here show that "plastic antibodies" prepared by molecular imprinting offers a potential solution to this problem. This technique fuses the advantages of synthetic molecularrecognition-elements and biological receptors, to result in molecularly imprinted polymers (MIPs) with receptor like binding affinity. Recently we reported on MIPs recognizing tyrosine or serine phosphorylations for biased enrichment of peptides from cancer cells or cerebrospinal fluid (CSF) respectively. We here present a proof of concept demonstration showing that MIPs can capture phosphopeptides in a sequence specific manner in highly complex biomedia. This can in principle be translated into robust, low cost multiplex assays with a potential impact on clinical phosphoproteomics.
\end{abstract}

\footnotetext{
${ }^{1}$ Department of Biomedical Science, Faculty of Health and Society, Malmö University, 20506 Malmö, Sweden

2Department of Biochemistry and Molecular Biology and VILLUM Center for Bioanalytical Sciences, University of Southern Denmark, DK-5230 Odense M, Denmark
} 
Proteomic relies on the quantitative analysis of proteins, protein-protein interactions, worldwide mapping of protein complexes. ${ }^{1,2}$ One of the most substantial research of proteomics is based on the identification and analysis of post-translational modifications (PTMs). The general steps involved in MS-based proteomics was shown in Scheme 1a including extracting proteins from bio samples, followed by digest and identify by MS analysis to obtain proteome database. Protein phosphorylation (covalent attachment of phosphate group to aminoacid(s)) is known as one of the ubiquitous PTM among identified chemical alterations. ${ }^{3-5}$ Phosphorylation is a natural and dynamic process, which is controlled by the reversible mechanism of phosphatases/kinases enzymes. ${ }^{6}$

It has been estimated that there are around $30 \%$ of proteins that are phosphorylated in eukaryotic organism ${ }^{7}$ and 518 kinases $^{8}$ and about 150 protein phosphatases ${ }^{9}$ were identified in the human genome. Phosphorylation mostly occurs on three main aminoacid residues, which are serine (Ser), threonine (Thr), and tyrosine (Tyr) in the level of $86.4 \%, 11.8 \%$, and $1.8 \%$ for pSer, pThr, and pTyr, respectively ${ }^{10}$. The attachment of phosphate groups to amino acids causes changes such as increasing hydrophilic property resulting in conformational change of the whole protein. These changes play a vital role in signal transduction and the regulation of many biochemical processes such as cell proliferation and differentiation ${ }^{11}$ as a consequence of diversity in cellular signaling pathway such as activity and interaction of proteins, mostly related with human disease status. ${ }^{12-15}$ Several diseases, notably cancer, are associated with in the protein phosphorylation signaling networks and consequently, drugs predominantly target protein kinases. ${ }^{16}$

ZAP-70 ( $\xi$-associated protein of $70 \mathrm{kDa})$ is cytoplasmic tyrosine kinase identified as tyrosine phosphoprotein in T-cell antigen receptor (TCR) signaling. ${ }^{17,18}$ The TCR simulation in the localization and activation of ZAP-70 is based on the downstream of protein tyrosine kinases (PTKs) which is shown in Scheme 1b. ${ }^{17-21}$

The transferring of extracellular signals to intracellular structure due to the engagement of TCR with major histocompatibility complex (MHC) introduces T-cell activation pathway. ${ }^{19,22,23}$ This pathway is controlled by the invariable polypeptide chains of one of the co-receptor in TCR signaling, CD3 (cluster of differentiation 3) complex which represents $\gamma, \delta, \varepsilon$, and $\zeta$ (non-covalently associated) chains. $^{24-26}$ This CD3 complex and $\zeta$ chain as cell signaling molecules comprise immunoglobulin receptor family tyrosinebased activation motif (ITAM) in the cytoplasmic tails. ${ }^{27-29}$ This conserved motif consists of two tyrosine sides which are subunits of SFK (Src family kinase) and Lck (lymphocyte-specific PTK), as a member of SFK, positioned on CD4 surface protein ( $T$ helper cell) activates the phosphorylation of tyrosines on ITAM which utilizes the recruitment of other proteins such as ZAP-70 onto phosphorylated tyrosine motif. ${ }^{19,21,30}$ The phosphorylation on ZAP-70 mediated by Lck leads to the kinase activity of ZAP-70 and its autophosphorylation. ${ }^{31-35}$ These effects are most likely associated with the structural changes in the proteins induced by the phosphorylation. Activated ZAP-70 further engage with immunoreceptors of $T$ lymphocytes and phosphorylate other crucial proteins such as SLP-76 (Src homology 2 (SH2) domain containing leukocyte protein of $76 \mathrm{kDa}$ ) which mediates diversity in downstream signaling pathway of TCR leading to cellular responses. ${ }^{19,36-39}$ ZAP-70 as cytoplasmic tyrosine kinase and its kinase activity in T cell is associated with disease states..$^{21,40}$ The overall structural organization of ZAP-70 is presented in Scheme 1c. 
a
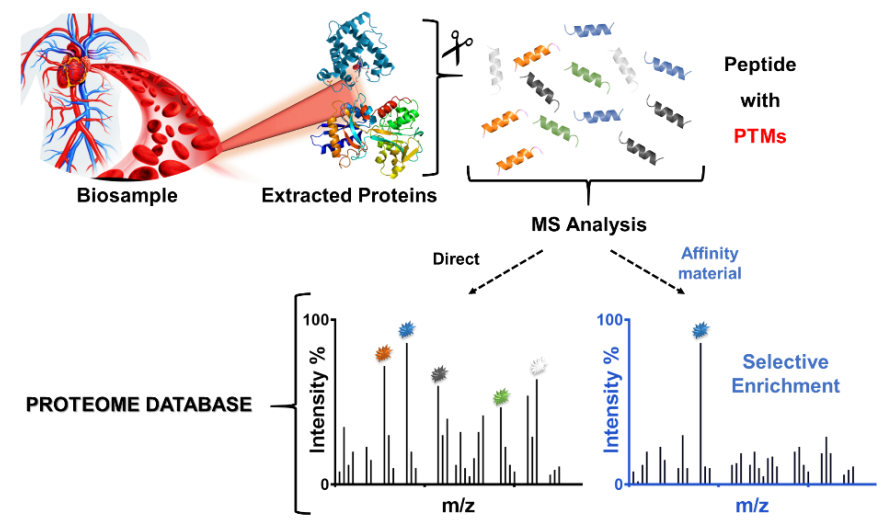

b

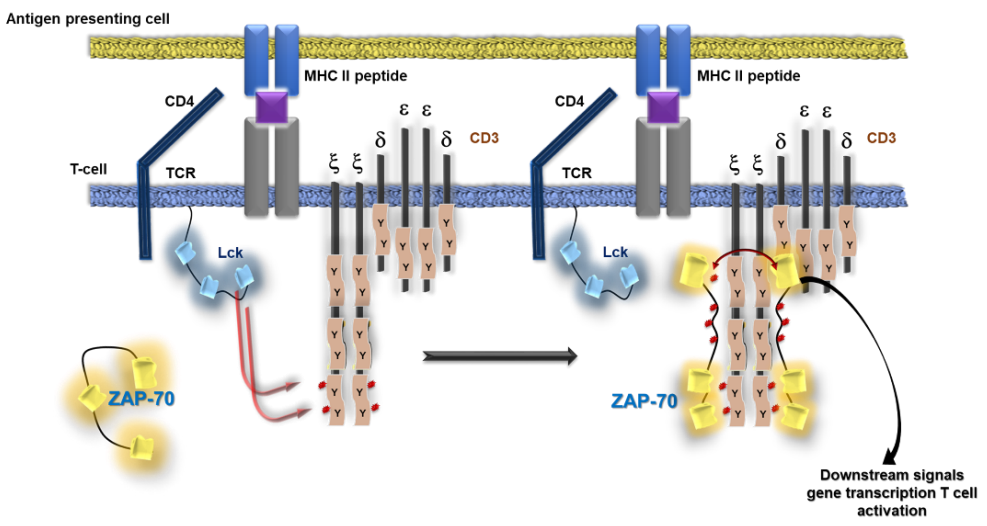

C
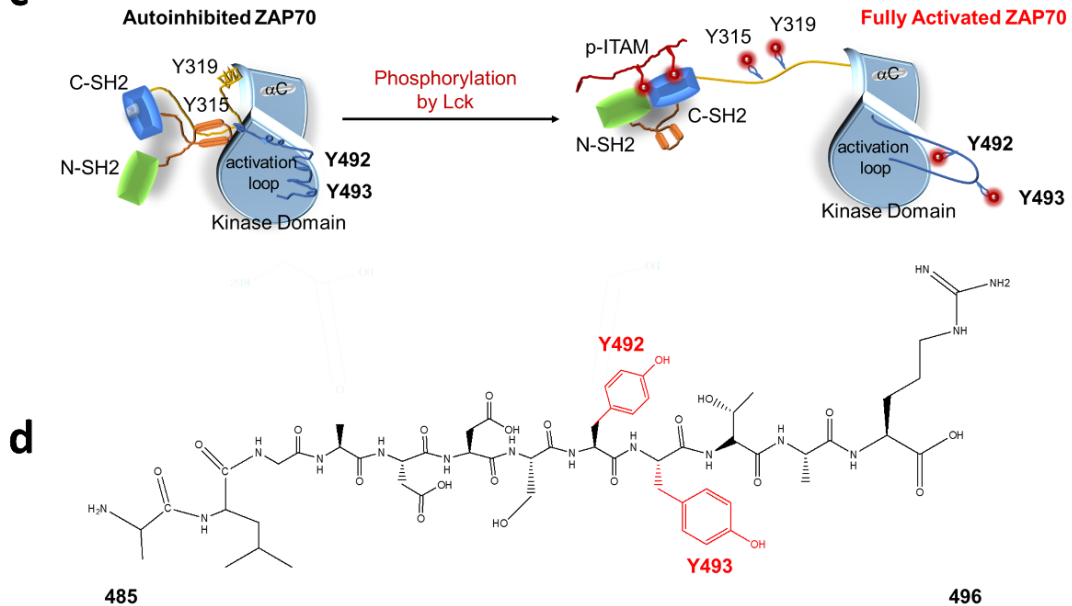

Scheme 1: $\mathbf{a}$. The general steps for identification of PTMs by MS analysis $\mathbf{b}$. The model for early steps of TCR signaling and the mechanism for activated phosphorylation on zeta-associated protein of $70 \mathrm{kDa}$ (ZAP-70) before downstream signaling pathway. The schematic had been adopted from the literature ${ }^{19} \mathbf{c}$. The domain organization of ZAP-70 and phosphorylated tyrosine residues, which are Y492 and Y493 in kinase domain and d. Sequence of the peptide (ALGADDSYYTAR) amino acids $485-496$ of ZAP-70 in the kinase domain. 
The structure of ZAP-70 consists of amino- and carboxyl- terminal $\mathrm{SH} 2$ domains (N-SH2 and $\mathrm{C}$ $\mathrm{SH} 2$ ) composed with interdomain (Y315, Y319) and kinase domain (Y492, Y493) in the activation loop. ${ }^{19}$ The tyrosine sites in interdomain are known as positive regulators which are initially phosphorylated by Lck and they act as hampering the reverse kinase mechanism in the auto-phosphorylation of kinase domain. ${ }^{41}$ Moreover, two tyrosine sites which are Y492 and Y493 are expressed in kinase domain considering the full peptide sequence represented in Scheme 1d.

Phosphorylation on these two tyrosine residues in the activation loop is found as the regulation of quantitative TCR signaling related to the prognostic values of chronic lymphocytic leukemia (CLL) disease identified as B-cell malignancy. ${ }^{42-44}$ In the identification of diseases and their stages, the membranebound immunoglobulin (Ig) receptor plays a vital role to understand the B-cell development. ${ }^{45}$ In the analysis of normal and malignant B-cell, heavy chain variable genes (IgVh) express the profile of patient disease states in the field of B-CLL. ${ }^{43,46-48}$ However, some studies have shown that CLL cells in aggressive/indolent disease not only related with the mutated/unmutated IgVh gene but also correlated by presence/lack of ZAP-70 expression. ${ }^{47,49}$ The identification of ZAP-70 signaling in B-cells is based on the regulation for CLL and/or regulation of mutational structure of IgVh gene which makes the phosphorylation of ZAP-70 expressed in B-cell as strong candidate for early treatment of CLL disease.

Since kinases are present in low abundance and phosphorylation is often non-stoichiometric, the elucidation of regulation mechanism is vital and requires analytical methods that can identify specifically the phosphorylation site(s) in small amounts of proteins. The current analytical strategies for the identification and enrichment of phosphorylated peptides and proteins, which are on immunoprecipitation, metal-oxide based affinity chromatography and chemical derivatization.

These techniques have their particular benefits for the extraction and analysis of phosphorylation with some drawbacks. Immunoprecipitation-based enrichment requires high quantity of sample ${ }^{50}$ with lowstability and non-reusability. Antibodies against pSer and pThr cause lack of side discrimination because of low-immunogenicity of these phospho-sites. Affinity chromatography based identification might show cross-reactivity with non-phosphorylated peptides dealing with acidic residues with low capacity and less selectivity from complex samples. In terms of chemical derivatization, $\beta$-elimination (based on facile dephosphorylation under alkali conditions) exploits unique property for the identification of phosphoamidate residues such as phosphorylation on histidine (pHis) over phosphoesters such as pSer and pThr; however, pTyr is not dephosphorylated in alkaline environment and this situation causes limitation on enrichment efficiency.

In this manner, smart capture phases based on molecularly imprinted polymer (MIP) technology $\mathrm{y}^{51,52}$ is an emerging field of research which fuses the advantages of synthetic molecular-recognition-elements with biological receptors, recently targeting higher molecular-weight analytes ${ }^{53-59}$ such as peptides and proteins as well. Many studies have been reported to develop phosphopeptide selective MIPs. In terms of the level of phosphopeptide discrimination, the validation of phospho-residues over nonphosphorylated ones and the identification of different phospho-sites using controlled bias materials increase the attention on imprinting technology to design robust, low-cost, and non-immuno-based capture materials. 
a

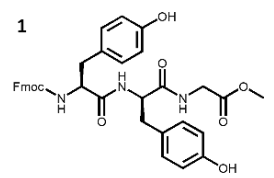

Fmoc-Tyr-Tyr-Gly-OMe

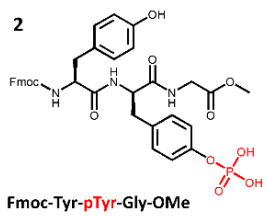

3

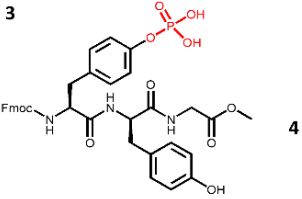

Fmoc-pTyr-Tyr-Gly-OMe

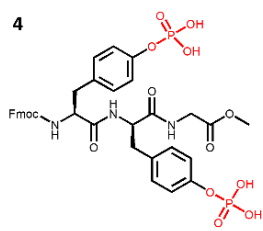

Fmoc-pTyr-pTyr-Gly-OMe

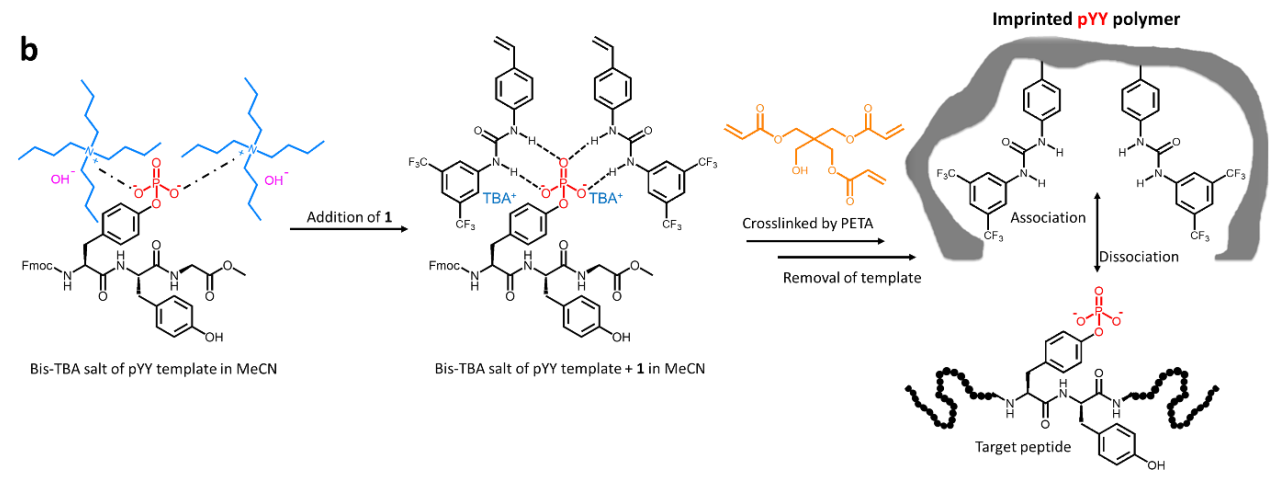

Scheme 2: a. Synthesized N- and C- protected non-phosphorylated and phosphorylated templates. b. Synthesis protocol of imprinted polymers and rebinding mechanism target-pYY-imprinted polymer was given as example-

In this paper, a study was reported targeting on phosphorylation of epitope tripeptides of ZAP70 , which has the peptide sequence of (ALGADDSYYTAR) amino acids 485-496 in the kinase domain. This approach is based on a method relying on the use of molecularly imprinted polymers to design smart capture phases for ZAP-70 phosphopeptides enrichment using sequence specific pY-MIPs due to epitope imprinting idea of four $\mathrm{N}$ - and $\mathrm{C}$ protected templates (Scheme 2a, see SI for synthesis of templates and characterization Figure S1a) together with neutral urea based receptor (Scheme $\mathbf{2 b}$ ).

In the study, regarding to the target pY-ZAP-70, four imprinted polymers were prepared us-ing the synthesized $\mathrm{N}$ - and $\mathrm{C}$ - protected templates: YpY MIP (targeting pY493), pYY MIP (targeting pY492), pYpY MIP (targeting pY492pY493), and YY MIP as control for the following sequence,
ALGADDSY ${ }^{492} Y^{493} T A R$. The proposed method allows to clearly extracting of phosphopeptides (p-peptides) with specifically targeting site selective or stoichiometric enrichment from a complex tryptic digest with lower spiking level. Considering pTyr modification and the other level of selectivity such as region- or stoichiometric selectivity for the development of effective diagnostics in early stage and treatment of diseases, this study is a proof-ofconcept using imprinting approach for synthesis of artificial binders and the results presented in the enrichment of target $p$ peptide from complex mixture showed the potential use of imprinting approach to design sequence specific receptors, which are capable of enrichment of phosphorylated residues in other levels of selectivity. 


\section{Results}

Probing affinity and selectivity of polymers in peptide mixture. To evaluate the recognitions of imprinted polymers, we firstly assessed their abilities to bind their corresponding templates (Fig. S1b) which confirm biased selectivity of binders towards corresponding pTyr targets and followed testing of deca-peptides (Fig. 1). For this aim, the polymers were incubated with the mixture of four peptides prepared in various $\mathrm{MeCN} / \mathrm{H}_{2} \mathrm{O}$ mixtures buffered with $0.1 \%$ three different modifiers: TFA, FA, or TEA. In general, traditional enrichment protocols for phosphopeptide enrichment is based on acidic treatment to eliminate binding of acidic amino acids such as carboxylic acid containing peptides. In here, three different modifiers were used to see the effect of $\mathrm{pH}$ in the binding of peptides towards affinity materials.

The bound percent of target phosphopeptide was further divided by the bound percent of non-target phosphorylated / nonphosphorylated peptides to calculate the selectivity ratio of target peptide to the corresponding polymer (Fig. 1, Fig. S2) - for instance YpY MIP targets GADDSYpYTAR peptide, the ratio of binding percent of GADDSYpYTAR(YpY) over GADDSYYTAR (YY) gives how much YpY MIP has preference on YpY peptide over YY peptide.

The target peptide showed significant selectivity towards non-target peptides when the binding was carried out in MeCN-rich content modified with $0.1 \%$ TFA (Fig. 1a, b, c). In contrast, the selectivity ratio of target peptide found lower and inconsistent when water-rich media or other modifiers, FA and TEA, used in binding. Since here, we are dealing with $\mathrm{H}$-bond complex, which occurs via the interaction between urea groups in macromolecular scaffold and prosthetic phosphate residue in peptides, the protonation state of phosphate plays critical role in affinity towards binding cavities. Given that the basicity of employed binding solution increases from TFA to TEA, the protonation degree of phosphate group changes from monoanion to dianion. Complete deprotonation of phosphate
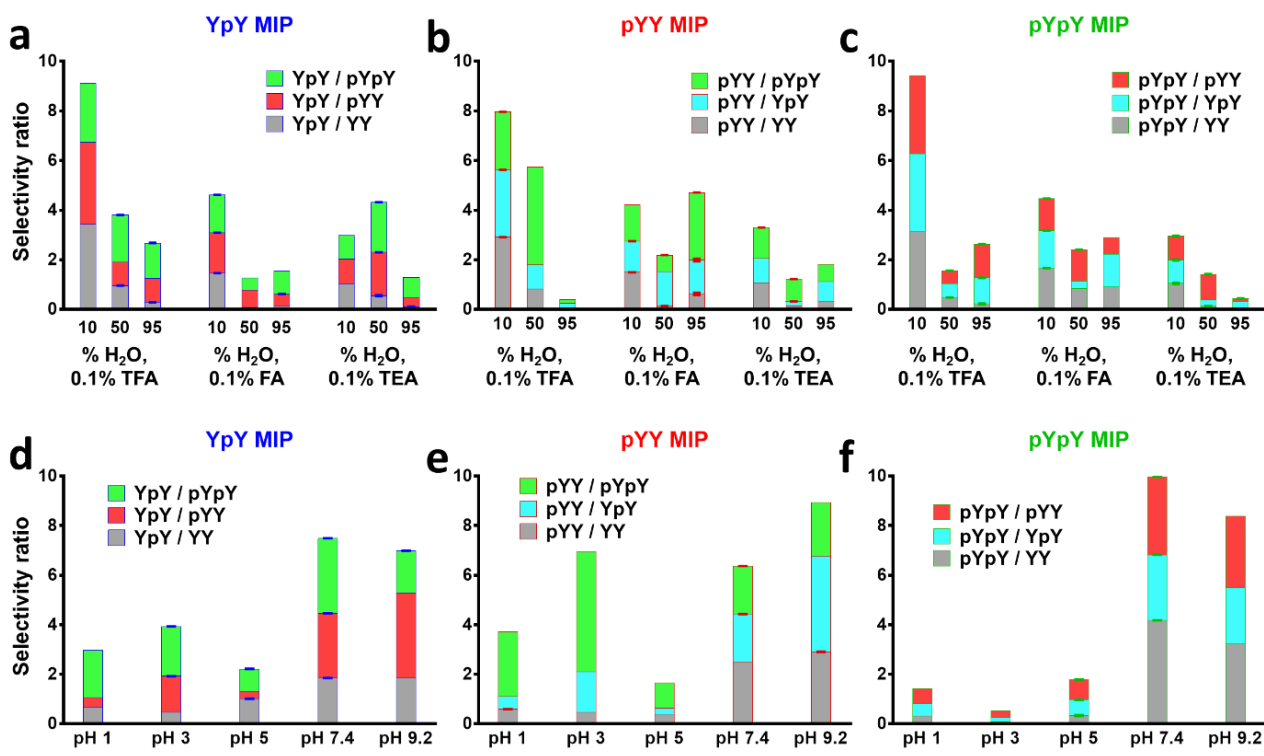

Figure 1: The selectivity ratio of phosphopeptide-targeting imprinted polymers. Testing completed in $10 \%, 50 \%$, and $95 \% \mathrm{H}_{2} \mathrm{O}+0.1 \%$ TFA, FA, or TEA modifiers for a YpY b pYY c pYpY MIP. Testing completed in aqueous buffers at different $\mathrm{pH}=1.0,3.0,5.0,7.4$, and 9.2 for $\mathbf{d}$ YpY e $\mathrm{pYY} \mathbf{f} \mathrm{pYpY}$ MIP. Labels represent target/non-target. pYpY = GADDSpYpYTAR, $p Y Y=G A D D S p Y Y T A R, Y p Y=$ GADDSYpYTAR, and $Y Y=$ GADDSYYTAR. Error bars for triplicate. 
group might be enhanced the formation of more $\mathrm{H}$-bonds but the pronounced bias towards target peptide could no longer be observed (Fig. S2g). Addition to this, we noted that the adsorbed amount of peptides decreases with increasing amount of water presented in the binding solution (Fig. S2). The lack of efficient recognition in water-rich media is due to highly hydrated form of phosphate anion that weakens the interaction with urea groups in receptor. Moreover, due to less hydrophilicity of non-phosphorylated peptide compared to phosphorylated ones promotes the non-specific contribution of $Y Y$ peptide at 95\% water present in binding solution (Fig. S2c, f, i).

In order to achieve, specific recognition of target phosphopeptides in water media, the polymers were later on incubated with the peptide mixture prepared at aqueous buffers at different $\mathrm{pH}=1.0,3.0,5.0,7.4$, and 9.2. In here, the purpose is to address destabilizing effect on the interactions -due to hydrated state of phosphopeptides in water- by presenting ionic strength media.

The selectivity ratio of target peptide over nontarget peptides was calculated as described before. The presence of salts in aqueous media had positive effect on selective binding of target peptide at $\mathrm{pH}=7.4$ and 9.2 compared to acidic pH (Fig. 1d, e, f). This is most likely related to the different protonation states of phosphate anion and the contribution of salt concentration on hydrated shells. The phosphate group is fully deprotonated at neutral or basic $\mathrm{pH}$. The presence of salts in aqueous media stabilize hydrated peptides due to reducing surface tension by decreasing electrostatic energy among peptide molecules. This leads increase on hydrophobic solubility and will help carrying peptides towards binding cavities when the mixture treated with $\mathrm{pH} 7.4$ or 9.2. In acidic $\mathrm{pH}$, phosphate is fully or singly protonated. The more hydrophobicity created the higher interaction with urea moiety established but diminished the target selectivity (Fig. S3).

To highlight the contribution of sequence specific binding, the results obtained from $Y Y$ MIP (prepared using non-phosphorylated template in the synthesis) was taken into account. Similar binding profile obtained using YY MIP for each peptide proves that how the lack of phosphate group in the synthesis of imprinted polymers effects polymer structure, binding cavities, and in turn target affinity exerted by receptor.

Adsorption isotherms and binding parameters of polymers. The binding-energy distributions of phospho-peptide targeting polymers are given by single-component adsorption in $90 \%$ $\mathrm{MeCN}+0.1 \%$ TFA and aqueous buffer at $\mathrm{pH}=7.4$ (Fig. 2). The corresponding polymers targeting $\mathrm{YpY}, \mathrm{pYY}, \mathrm{pYpY}$ peptides displayed a higher uptake of their target peptide compared to their non-target phosphorylated peptides (Fig. 2a, c). Expectedly, the uptake percent is much lower in buffer (Fig. 2b, d) than MeCNrich media -due to hydration of anions- still, the differential uptake clearly confirms the effect of imprinting. Fitting these data with the Langmuir mono-site binding model resulted curves and fitting parameters $K_{a}$ (association constant) and $B_{\max }$ (maximum binding in $\mu \mathrm{mol} / \mathrm{g}$ ) given in Table S1. The presence of much steeper slope before saturation point for target peptide by corresponding polymer confirms the affinity for sequence specific phospho-site recognition with tight binding. The preference for target peptide is reflected in higher association constants recorded for target peptide with higher binding maxima. The association constants for target peptide was found in $\mu \mathrm{M}$ range in both binding solution that exceed the anion affinity for most reported neutral receptors such as antibodies with respect to anion binding especially in aqueous buffers ${ }^{60,61}$. In contrast, binding isotherms of non-target peptides displayed shallower curves and reach to binding maxima immediately and association constant was 

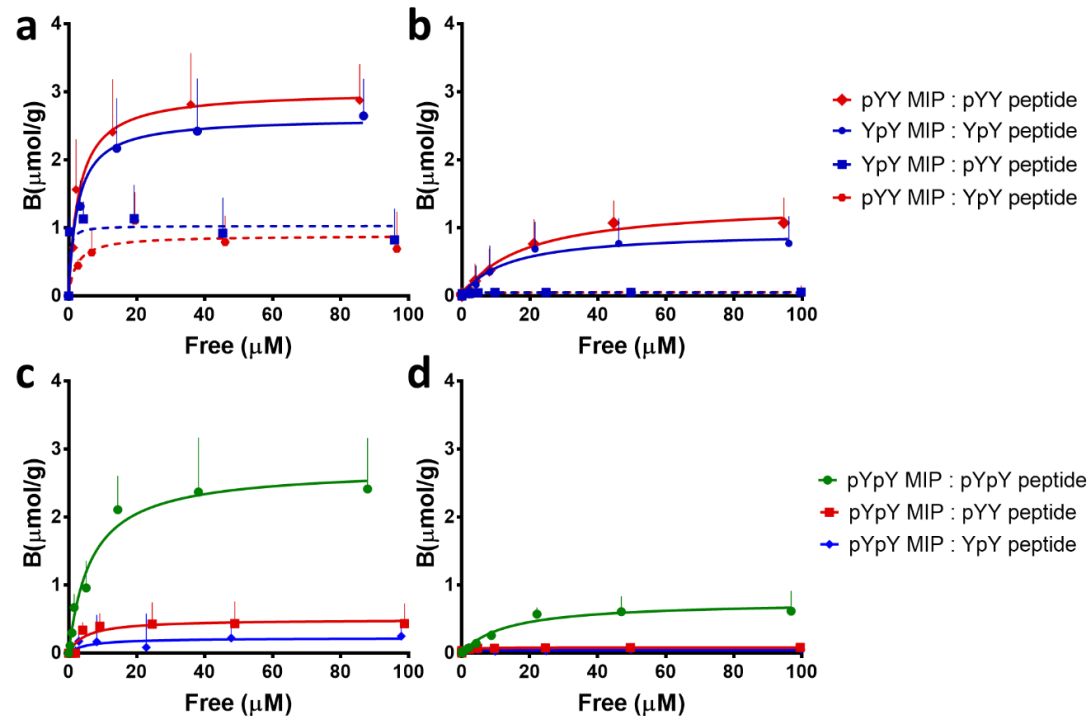

Figure 2: The binding-energy distributions of phospho-peptide targeting polymers. Equilibrium binding isotherms of YpY and pYY MIPs in a $90 \% \mathrm{MeCN}+0.1 \%$ TFA, $\mathbf{b}$ Aqueous buffer at $\mathrm{pH}=7.4$. Equilibrium binding isotherms of $\mathrm{pYpY}$ MIP in c $90 \% \mathrm{MeCN}+0.1 \%$ TFA, d Aqueous buffer at $\mathrm{pH}=7.4$. pYY $=$ GADDSpYYTAR, YpY $=$ GADDSYpYTAR and pYpY $=$ GADDSpYpYTAR.

found around hundred times less compared to $\mathrm{K}_{\mathrm{a}}$ values of polymers with respect to target peptide. These all contrast with the results for YY MIP - where non-phosphorylated template used in the synthesis. The isotherms obtained from YY MIP displayed no preference on any peptide with immediate saturation.

Enrichment of spiked phosphorylated ZAP-70 deca-peptides from complex protein digest mixture. Having proven the binding and affinity performance of imprinted polymers on distinguishing stoichiometric and/or site selective phosphate groups in the same sequenced peptide, further investigation is required to understand their ability to enrich target $p$-peptide from complex mixture. For this aim, we tested the performance of imprinted polymers for $\mathrm{p}$-peptide enrichment using the mixture of ZAP-70 deca-peptides spiked with twelve protein digests according to the workflow. Fig. 3 (spiking level is 1:10) and Fig. S5 (spiking level is $1: 100$ ) show the extracted chromatograms of the preenrichment control (reference, R) sample and the post-enrichment (flow through + wash,
$\mathrm{FT}+\mathrm{W}$ and elution, E) fractions using $\mathrm{p}$-peptide targeting imprinted polymers.

First we noted that matrix-related peaks (shown in light pink) showed reduction in the post-enrichment fractions compared to reference. This means that non-specific contributions of peptides from complex protein digest were eliminated during loading and washing steps. The abundance of target peptide over non-target peptides in the elution fractions (Fig 3a, d) compared to preenrichment samples (Fig $\mathbf{3 c}$, f) provide unequivocal evidence for the presence of site selective discriminative binding sites. In terms of targeting double phosphorylated peptide, the abundance of $\mathrm{pYpY}$ peptide compare to YpY and pYY peptides did not look clearly pronounced from elution fraction (Fig $\mathbf{3 h}$ ). However, when the relative peak area ratio (ApYpY / AYpY and ApYpY / ApYY) in E fractions compared to $\mathrm{FT}+\mathrm{W}$ fractions obtained using pYpY MIP, it was found that while single phosphorylated peptides were 10 times higher in FT + W than target, pYpY peptide showed 10 times higher intensity in the elution fraction than non-targets. This switching behavior 

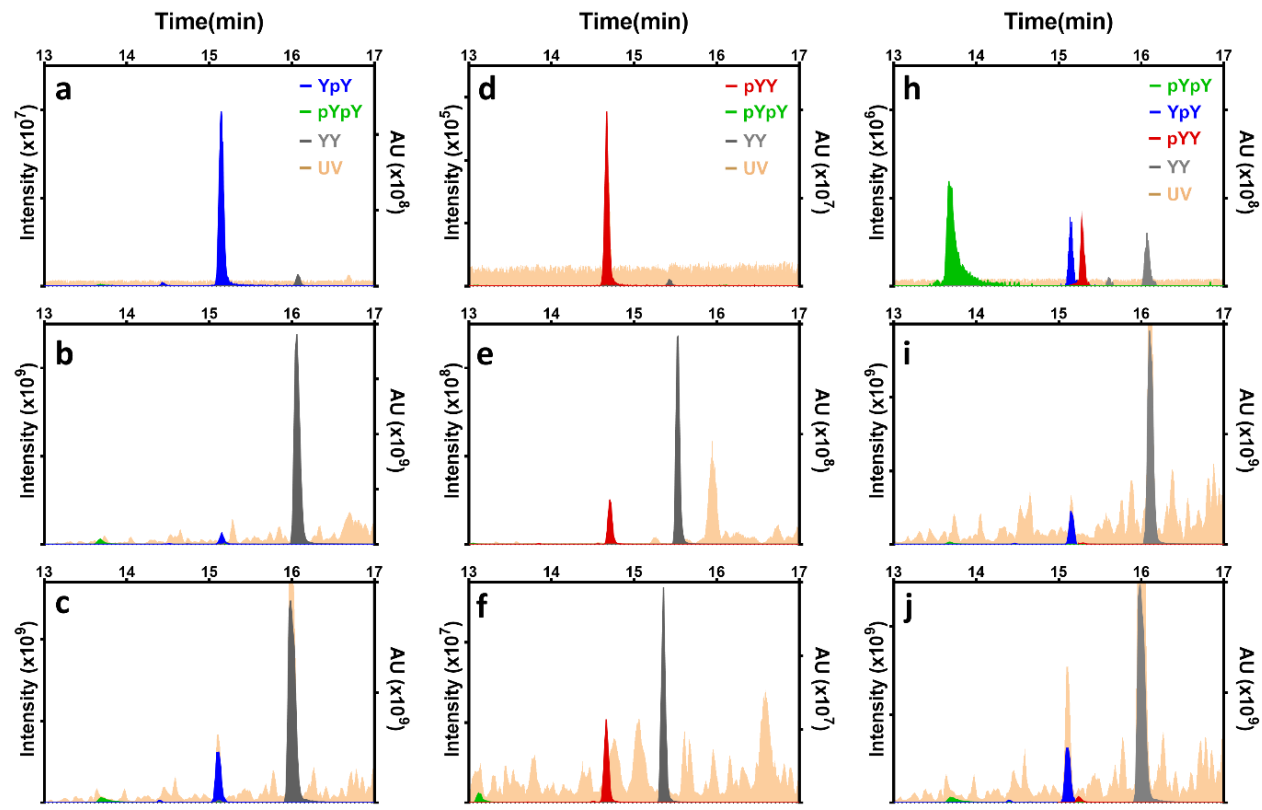

Figure 3: Extracted LC-MS chromatogram of highlighting spiked ZAP-70 peptides (indicated in colors) corresponding to fractions after enrichment. a YpY-MIP E, b YpY-MIP FT+W, c YpY-MIP R, d pYY-MIP E, e pYY-MIP FT+W, f pYY-MIP R, $h$ pYpY-MIP E, i pYpY-MIP FT+W, j pYpY-MIP R. E = Elution, $F T+W=$ flow-through + loading, $R=$ reference $p Y p Y=$ GADDSpYpYTAR (green), YpY = GADDSYpYTAR (blue), pYY = GADDSpYYTAR (red), and YY = GADDSYYTAR (gray). The light pink chromatogram in the background stands for full chromatogram. The left $y$-axis shows intensity of spiked peptides, the right $y$-axis refers for the intensity of full chromatogram.

confirms the possibility to extract target $p$ peptides from complex mixture especially at 1:10 spiking level. Even though the contribution of non-phosphorylated peptide increased at 1:100 spiking level (Fig S4), the selective enrichment of single phosphorylated YpY and pYY peptides were clear. On the other hand, the elution fraction obtained by $\mathrm{pYpY}$ MIP (Fig S4h) showed reduced selectivity on target peptide. The calculated preference ratio of $\mathrm{pYpY}$ peptide over non-target peptides ((Atarget / Anon-target) $)_{\mathrm{E}}$ divided to (Atarget /

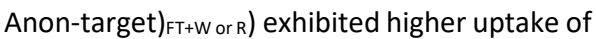
pYpY peptide, which proves the high-affinity sites generated for particular imprinted polymer compatibility with complex matrices.

Enrichment of ZAP-70 phosphopeptides obtained by stimulated Jurkat T-cells. ZAP-70 non-receptor protein tyrosine kinase is implicated as controlling drive for signaling via $\mathrm{T}$ cell antigen receptors. The genetic abnormalities in ZAP-70 expression are extremely correlated with immunodeficiency. The overexpression of ZAP-70 in B cell chronic lymphocytic leukemia (B-CLL) is related with the progress of the disease. Subsequent gene expression profiling showed that ZAP-70 expression was the most discriminating feature between the two subtypes. ${ }^{62}$ In fact, ZAP-70 protein expression in $\mathrm{B}-\mathrm{CLL}$ patients appear to have more predictive value than $\mathrm{IgVh}$ mutations, and its expression level appears to be constant during the course of disease ${ }^{47}$ The phosphorylation of ZAP-70, instead, is associated with B-cell receptor signaling as negative prognostic factor on the disease.

Having determined that the sequence specific imprinted polymers exhibited promising characteristic for enrichment on custom-made ZAP-70 peptides, we set out to further study on the enrichment of ZAP-70 p-peptides extracted from stimulated Jurkat T-cells, a human leukaemic $\mathrm{T}$ cell lymphoblast cell line. 
First, a number of experiments on stimulation of cells were performed. $\mathrm{CD} 3, \mathrm{H}_{2} \mathrm{O}_{2}, \mathrm{Na}_{2} \mathrm{VO}_{4}$, $\mathrm{CD} 3+\mathrm{H}_{2} \mathrm{O}_{2}$, and $\mathrm{CD} 3+\mathrm{Na}_{2} \mathrm{VO}_{4}$ combinations were used for cell treatment. Next, the overexpression of phosphorylation on either Y492 or Y493 due to different stimulations was confirmed using anti ZAP-70 phosphoantibodies. After profiling and identification of the overexpressed ZAP-70 phosphoproteins, finally, in-gel proteolytic digest was performed to cleave $p$-proteins and extract peptides from digested protein for further enrichment experiments (Scheme. S2).

For the stimulation, different regulators and their combinations were employed. CD3 was used as a protein complex involved in activating T-cell receptor signaling where phosphorylated and activated tyrosine kinase ZAP-70 is recruited. ${ }^{63}$ Signaling function of CD3 stimulates TCR as well as tyrosine kinase activity. $\mathrm{H}_{2} \mathrm{O}_{2}$ as reactive oxygen species, which activates the response of extracellular receptor kinase via oxidative stress in T cells, initiates TCR and activate protein-tyrosine kinase ZAP$70 .{ }^{64} \mathrm{Na}_{2} \mathrm{VO}_{4}$ was used as an inhibitor of phosphotyrosyl protein phosphatases (PAP) activity, which helps and possibly increase the cellular levels of protein phosphorylation. ${ }^{65}$

The expression of phosphorylation on $\mathrm{Y} 492$ and Y493 - placed in ALGADDSSYYTAR sequence in kinase domain- using different stimulation was shown in Fig. 4a. Moreover, the raw band intensity values of target $p$-proteins in different stimulated samples plotted against the nonstimulated control sample and the comparison shows varying expression levels of the target protein in Fig. $\mathbf{4 b}$.

First, we observed the non-stimulated control sample has constitutive hyperphosphorylation of Y493. Since Jurkat leukemic T-cell line was utilized in this study, the potential phosphorylation in the kinase activity of ZAP70 may function. The overexpressed phosphorylation of Y493 and mechanism on ZAP-70 catalytic activity were previously studied elsewhere. ${ }^{66}$ It was found that the phosphorylation of $\mathrm{Y} 493$ as positive regulatory mechanism ${ }^{67}$ which is required for activation of
ZAP-70 and lymphocyte antigen receptor function. ${ }^{68}$

CD3 stimulation on cells slightly increased the expression of $\mathrm{Y} 493$ phosphorylation compared to basal level in non-stimulated one while $\mathrm{Y} 492$ remained unchanged. On the other hand, $\mathrm{H}_{2} \mathrm{O}_{2}$ stimulation alone had no effect on both tyrosine; indeed, it caused slight decrease on Y493 phosphorylation.

However, the presence of either $\mathrm{H}_{2} \mathrm{O}_{2}$ or $\mathrm{Na}_{2} \mathrm{VO}_{4}$ and their combinations together with CD3 caused an increase in the accumulation of phosphorylation on Y492 induced by kinase activity while the phosphorylation on $\mathrm{Y} 493$ remained indistinguishable from nonstimulated or CD3-stimulated one.

Several previous studies showed that the catalytic activity of ZAP-70 kinase domain is related with the phosphorylation of tyrosine residues $\mathrm{Y} 493$ in the activation loop ${ }^{66,69,70}$ because $\mathrm{Y} 493$ phosphorylation allows the rearrangement of the activation loop which helps access the catalytic center for further activation of downstream signaling. On the other hand, Y492 phosphorylation is defined as negative regulator for ZAP-70 function and its biological role is still unclear. ${ }^{70}$ It was revealed that the phosphorylation on Y493 might follow by auto-phosphorylation at $Y 492 .^{71}$ This suggest that increase phosphorylation on $\mathrm{Y} 492$ stimulated by presence of either $\mathrm{H}_{2} \mathrm{O}_{2}$ or $\mathrm{Na}_{2} \mathrm{VO}_{4}$ might not be related with the kinase activity of this particular phosphorylation itself, but might be related with the structural changes in ZAP-70 protein after kinase activity provided by phosphorylation on Y493. Since the phosphorylated Y493 promotes rearrangements in the conformation of active ZAP-70 and this can increase more accessibility of phosphate substrate towards phenolic hydroxyl groups with consequent enhance the activity of phosphate groups on $\mathrm{Y} 492$.

After conformation of phosphorylation on either $\mathrm{Y} 492$ or $\mathrm{Y493}$, only cell lysates stimulated with $\mathrm{CD} 3+\mathrm{H} 2 \mathrm{O} 2$ and $\mathrm{CD} 3$ were conducted for in-gel digest to obtain peptide mixture (see experimental). 

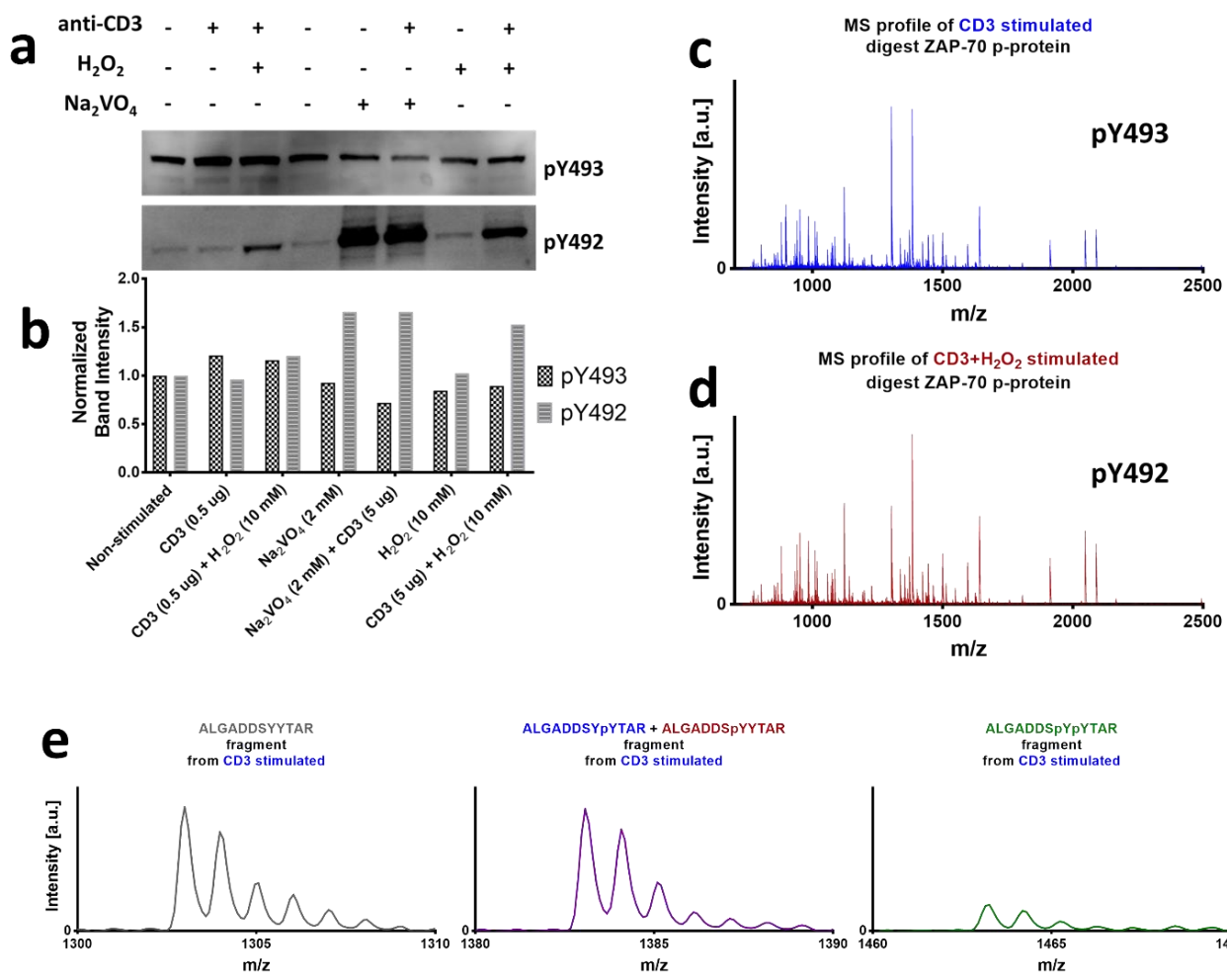

ALGADDSYPYTAR + ALGADDSPYYTAR fragment
from $C D 3$ stimulated

ALGADDSpYpYTAR from $\mathrm{CD} 3$ stimulated
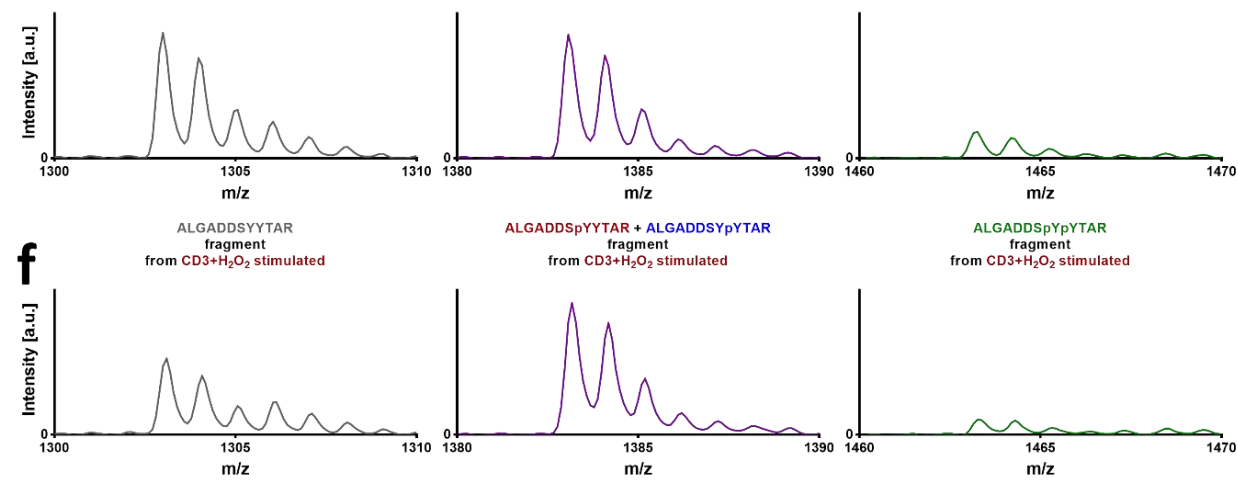

ALGADDSPYYTAR + ALGADDSYPYTAR fragment
from $\mathrm{CD} 3+\mathrm{H}_{2} \mathrm{O}_{2}$ stimulated ALGADDSPYPYTAR
fragment from $\mathrm{CD} 3+\mathrm{H}_{2} \mathrm{O}_{2}$ stimulated
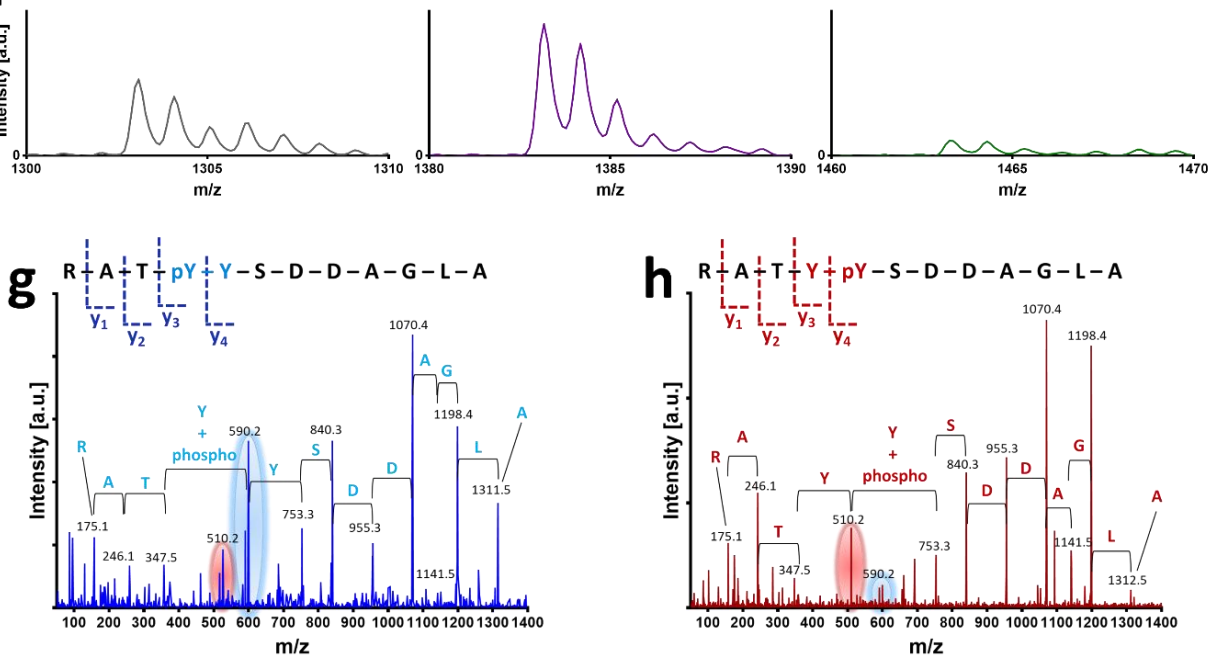

Figure 4: ZAP-70 phosphopeptides obtained by stimulated Jurkat T-cells. a Schematic workflow of stimulation of cells and extraction of proteins. $\mathbf{b}$ Western blot analysis of various stimulated cells with anti-phosphotyrosine antibodies (pY493 and pY492). MALDI/MS spectrum of digested ZAP-70 phosphoproteins stimulated by c only CD3, $\mathbf{d}$ combination of $\mathrm{CD} 3+\mathrm{H}_{2} \mathrm{O}_{2}$, $\mathbf{e}$ and $\mathbf{f}$ close-up of non-phosphorylated and phosphorylated peptides related with the target represented in the kinase domain sequence. LC-MS/MS fragmentation of $\mathbf{g}$ ALGADDSYpYTAR from CD3 sample, $\mathbf{h}$ ALGADDSpYYTAR from $\mathrm{CD} 3+\mathrm{H}_{2} \mathrm{O}_{2}$ sample. 
The full length of ZAP-70 YY (nonphosphorylated) protein (residues 1 - 619, Uniprot-P43403) was given in (see SI) and the peptide sequence in kinase domain was highlighted (residues 476-500). The MS-digest (cleavage by trypsin) list of possible short peptides of ZAP-70 YY (Table S2) and various phosphorylation states in the kinase domain namely: non-phosphorylated $\left(\mathrm{Y}^{0} \mathrm{Y}^{0}\right), \quad \mathrm{Y} 492$ phosphorylated $\left(\mathrm{Y}^{\mathrm{P}} \mathrm{Y}^{\mathrm{O}}\right), \mathrm{Y} 493$ phosphorylated $\left(Y^{0} Y^{P}\right)$, and both phosphorylated $\left(Y^{P} Y^{P}\right)$ with their mass-to-charge ratio was shown in Table S3. The sample complexity and peptide profiles of digested ZAP-70 p-proteins were screened using MALDI-MS (Fig. 4c, d). $Y^{0} Y^{0}$ (1302.5), $Y^{P} Y^{0}$ or $Y^{0} Y^{P}$ (1382.5) and $Y^{P} Y^{P}$ (1462.5) peptide sequences were identified. Particularly, the superimposed spectra shown in Fig. $4 e$ and $f$ stands for the peptides corresponding to residues 485-496 of human ZAP-70 in their different phosphorylation states.
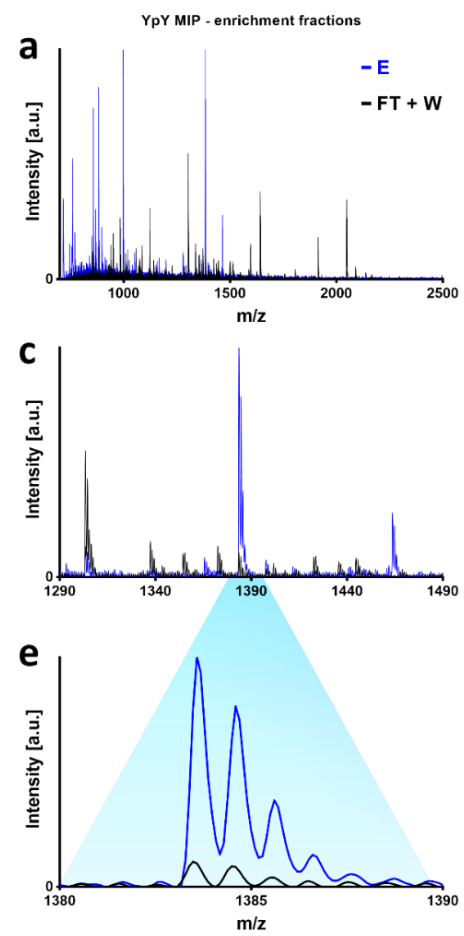

Confident phosphorylation site localization was achieved by LC-MS/MS fragmentation producing high sequence coverage and conserving the phosphorylation on fragment ions (Fig. 4g, 4h, Fig. S5). Comparison of fragmentation patterns of single p-peptides, the corresponding fragments peaks (indicated $\mathrm{y}$-ions in Fig. 4g, 4h) for ALGADDSYpYTAR (m/z $=590.2)$ and ALGADDSpYYTAR $(\mathrm{m} / \mathrm{z}=510.2)$ were confirmed the overexpressed phosphosites.

\section{Enrichment of p-peptides from natural extract} For the enrichment of p-peptides obtained from stimulated digest, we tested the performance of YpY MIP and PYY MIP with peptide mixtures obtained from $\mathrm{CD} 3$ and $\mathrm{CD} 3+$ $\mathrm{H}_{2} \mathrm{O}_{2}$ stimulated digest, respectively. MALDI$\mathrm{MS}$ profiles of $\mathrm{FT}+\mathrm{W}$ (Flow through+washing) and $E$ (elution) fractions obtained from enrichment using YpY and pYY MIPs shown in Fig. 5.
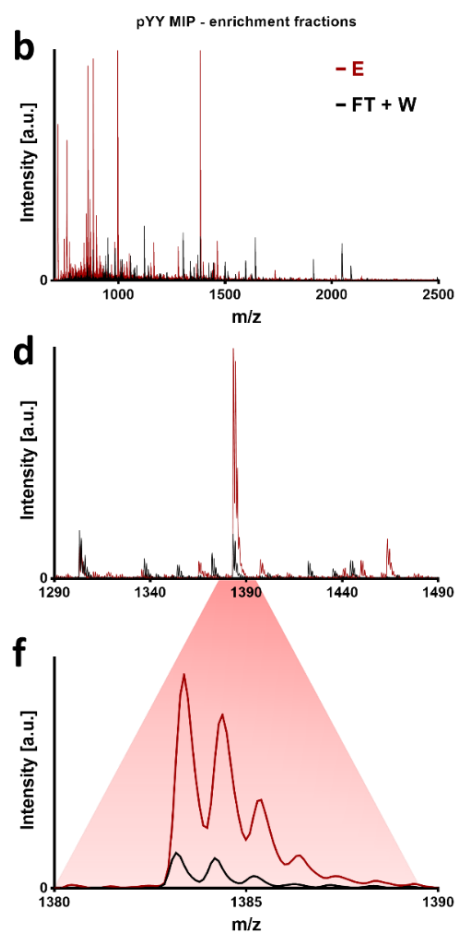

Figure 5: Enrichment profile of YpY and pYY MIPs. MALDI-MS spectrum of Flow Through + Wash (FT+W) and Elution (E) fractions obtained from enrichment using a YpY MIP and $\mathbf{b}$ pYY MIP, $\mathbf{c}$ and $\mathbf{d}$ close up for assigned peptides (YY, YpY or $\mathrm{pYY}$, and $\mathrm{pYpY}$ ) and $\mathbf{e}$ and $\mathbf{f}$ close up for target $\mathrm{p}$-peptide either $\mathrm{YpY}$ or $\mathrm{pYY}$. 
As can be seen in Fig. 5 and Fig. S6, the abundance of peptides is lower in $E$ fractions compared to $\mathrm{FT}+\mathrm{W}$ fractions. In the comparison of target short peptide region between $1290-1490 \mathrm{~m} / \mathrm{z}$ range where none (1302.5), single (1382.5) and double (1463.5) phosphorylated peptides can be detected (Fig. $\mathbf{5 c}$ and $\mathbf{5 d}$ ), non-phosphorylated peptides was found in FT+W fraction while the abundance of phosphorylated peptides were found more pronounced in the $E$ fraction (Fig. 5e and $\mathbf{5 f}$ ).

\section{Discussion and Outlook}

Overall, in this study, sequence specific pTyrtargeting imprinted polymer were synthesized regarding to the target ZAP-70 which is prognosticator for CLL disease. The presented proof-of-concept results showed that synthetic receptors synthesized by imprinting approach are capable of enrichment target $p$-peptide in sequence specific manner from complex mixture at very low spiking level. This level of selectivity discrimination via artificial receptors would lead in diagnostic applications in the case of biomarker discovery after completing more experiment on enrichment of target $p$ peptides from natural extract, optimization of enrichment conditions, testing all polymers and conclude the particular selectivity achieved using these binders.

\section{Experimental Section and Methods}

Preparation of Imprinted Polymers. The bistetrabutylammonium (bis-TBA) salt of Fmoc-Tyr-pTyr-GlyOMe, Fmoc-pTyr-Tyr-Gly-OMe (0.25 mmol) and FmocpTyr-pTyr-Gly-OMe (0.125 mmol) as a template, urea functional monomer (1) $(0.5 \mathrm{mmol})$, pentaerythritol triacrylate (PETA) (13.3 $\mathrm{mmol})$ as crosslinker (CL), acetonitrile (MeCN) (6.1 $\mathrm{mmol})$ as a solvent and azobis(2,4-dimethyl)valeronitrile (ABDV) (1\% w/w of total monomer amount) as initiator, respectively. For preparation of YY MIP, Fmoc-Tyr-Tyr-Gly-OMe as a template was used otherwise followed exactly same procedure as above. The each prepared solution for the polymerization was purged with a flow of nitrogen gas for $5 \mathrm{~min}$. and the polymerization was initiated by placing the solution containers in a water bath heated to $50 \mathrm{oC}$ for overnight. Each container was removed after polymerization and the polymers were crushed, and washed by $\mathrm{MeOH}: 1 \mathrm{~N} \mathrm{HCl}(1: 1 / v: v) \times 3$ times and $\mathrm{MeOH} \times$ 2 times for the removal of template. This process was followed by sieving to obtain the particles between $25 \mu \mathrm{m}$ and $50 \mu \mathrm{m}$ to use the polymers for further analysis.

Binding Test Using Short Peptides. Each polymer (10 mg) was suspended in $1.0 \mathrm{~mL}$ of a mixture of equimolar GADDSYYTAR, GADDSYPYTAR, GADDSPYYTAR, and GADDSpYpYTAR (each $20 \mu \mathrm{M}$ ) prepared in various ratios of MeCN:H2O buffered with $0.1 \%$ TFA, FA, or TEA. The same test was also completed using aqueous buffers at different $\mathrm{pH}=1.0,3.0,5.0,7.4,9.2$. Each suspension was shaken vigorously for $2 \mathrm{~h}$ to provide binding equilibrium and then each sample was centrifuged. The supernatant $(500 \mu \mathrm{L})$ was dried by using Genevac EZ-2 evaporator, then reconstituted in $95 \% \mathrm{H}_{2} \mathrm{O}+0.1 \%$ TFA $(200 \mu \mathrm{L})$, and analyzed by reversed phase HPLC. The column was Prodigy $5 \mu \mathrm{m}$ ODS-3 $100 \AA$ (Phenomenex, $150 \times 4.6 \mathrm{~mm}$ ). Mobile phases were (A) $100 \% \mathrm{H}_{2} \mathrm{O}+0.1 \%$ TFA and (B) $100 \% \mathrm{MeCN}+0.1 \%$ TFA. A linear gradient method of $5 \%$ $B$ to $20 \%$ B in $10 \mathrm{~min}$ at a flow rate of $1.5 \mathrm{~mL} / \mathrm{min}$. was used. The injection volume was $100.0 \mu \mathrm{L}$ and the detection was performed by UV absorbance measurement at $275.0 \mathrm{~nm}$. All experiments were performed in three parallel replicas.

Binding Isotherms. The imprinted polymers (10.0 mg) were separately suspended in $1.0 \mathrm{~mL}$ solution of different concentrations $(0-100 \mu \mathrm{M})$ of the peptides (GADDSYYTAR, GADDSYPYTAR, GADDSPYYTAR, GADDSpYpYTAR) in 90\% MeCN + 0.1\% TFA and aqueous buffer at $\mathrm{pH}=7.4$. The vials were shaken for $2 \mathrm{~h}$ followed by centrifugation and quantification of unbound analyte by HPLC using the method described above. The amount of bound analyte per unit mass of polymer $(B)$ was calculated. The binding curves were constructed by plotting the graph $B(\mu \mathrm{mol} / \mathrm{g})$ corresponding to free concentration, Cfree, and were subsequently fitted in the GraphPad Prism 7 software (GraphPad Software, La Jolla, CA, USA) by Langmuir mono-site binding model in the following formula, which is $Y=B \max * X /(K d+X)$, to calculate Bmax and Ka.

LC MS/MS Analysis. ZAP70 peptides were spiked in 12 protein digest (Carbonic anhydrase, BSA, Ovalbumin, Alpha casein, Beta casein, Beta lacto globulin, RNaseB, Alcohol dehydrogenase, Myoglobin, Transferrin, Lysozyme, Alpha amylase) with two different spiking level: 1:10 (1 pmol of peptide mix:10 pmol digest) and 1:100 (100 fmol of peptide mix:10 pmol digest). Each solution was prepared in $90 \% \mathrm{MeCN}+0.1 \%$ TFA. The polymer particle $(10 \mathrm{mg}$ ) was packed in single fritted SPE cartridges (ISOLUTE, Biotage) and was protected with a frit on top. In the enrichment protocol, the polymers were conditioned by using $90 \% \mathrm{MeCN}+0.1 \%$ TFA $(3 \times 1 \mathrm{~mL})$ followed by loading the spiked solution ( $1 \mathrm{~mL}$ prepared by $90 \% \mathrm{MeCN}+0.1 \% \mathrm{TFA})$. The washing step was performed by using loading solution ( $2 \times 0.5 \mathrm{~mL})$ and $100 \% \mathrm{H} 2 \mathrm{O}+$ 
$0.1 \%$ TFA $(2 \times 0.5 \mathrm{~mL})$, lastly the elution step was done by using two solutions, which were $80 \% \mathrm{MeOH}+0.1 \%$ TFA $(0.5 \mathrm{~mL})$ and $100 \% \mathrm{MeOH}+0.1 \% \operatorname{TFA}(0.5 \mathrm{~mL})$. In the regeneration, each SPE column was washed with following solutions: $80 \% \mathrm{MeOH}+20 \% \mathrm{HCl}(0.1 \mathrm{M})(3 \times 1$ $\mathrm{mL}), 100 \% \mathrm{H} 2 \mathrm{O}(3 \times 1 \mathrm{~mL})$, and $90 \% \mathrm{MeCN}+0.1 \% \mathrm{TFA}(3$ $x 1 \mathrm{~mL}$ ). Three fractions as loading (L), washing (W), and elution (E) were dried and elution fractions were used to test in LC-MS analysis. Each dried elution fraction for different spiking level with three replicas were tested in LC-MS analysis. Each fraction was firstly dissolved, vortexed and sonicated. The preparation of fraction for testing in LC-MS followed as: 1:100 elution fractions were dissolved in $18.8 \mu \mathrm{L}$ in $0.1 \% \mathrm{FA}(5.5 \mathrm{fmol} / \mu \mathrm{L})$ and then 2 $\mu \mathrm{L}$ of the solution was diluted in $9 \mu \mathrm{L}$ of loading solvent, 1:10 elution fractions were dissolved in $181.1 \mu \mathrm{L}$ of $0.1 \%$ FA $(5.5 \mathrm{fmol} / \mu \mathrm{L})$ and then $2 \mu \mathrm{L}$ of the solution was diluted in $9 \mu \mathrm{L}$ of loading solvent (each dilution process was estimated as $1 \mathrm{fmol} / \mu \mathrm{L}$ peptides exist). . Redissolved peptide samples were then analyzed on a Dione Ultimate 3000 RSLCnano system coupled online to an Orbitrap Fusion Tribrid mass spectrometer (Thermo Scientific). In the analysis of sample, $10 \mathrm{fmol}$ of each elution fraction (approximately) was injected in $10 \mu \mathrm{L}$ for $1 \mathrm{fmol} / \mu \mathrm{L}$ of peptides as expecting an elution of $100 \%$ recovery. This is the way to compare each elution fraction among each other. Additionally, two positive controls, which are 10.0 fmol injection of equimolar of ZAP70 peptides and 1:10 spiking solution in $10 \mathrm{fmol}$ of ZAP70 peptides spiked in $100 \mathrm{fmol}$ of protein digest, were analyzed. The LTQOrbitrap Velos was operated in positive ion mode with data-dependent acquisition. The full scan was acquired in the Orbitrap with an automatic gain control (AGC) target value of $1 \times 106$ ions and a maximum fill time of $500 \mathrm{~ms}$. Full-MS scans were acquired with resolution of 60000 fwhm followed by $10 \mathrm{MS} / \mathrm{MS}$ scans of the most intense ions, also acquired with a mass resolution at 15000 (HCD normalized collision energy $=35$; activation time $10 \mathrm{~ms}$ ). Raw data were viewed in Xcalibur v2.0.7 (Thermo Fisher Scientific, U.S.A.).

Stimulation of cells and protein analysis. Jurkat (ATCC TIB-152), were cultured under conventional conditions $\left(37^{\circ} \mathrm{C}\right.$, humidified atmosphere, containing $\left.5 \% \mathrm{CO}_{2}\right)$ in RPMI-1640 supplemented with 10\% FCS (Gibco), sodium pyruvate $(1 \mathrm{mM})$ and glucose $(4.5 \mathrm{~g} \mathrm{I} 1)$, penicillin and streptomycin. Before stimulation, cells were serum starved for 3 hours before stimulation. Thereafter cells were sedimented at $300 \mathrm{xg}$ for $5 \mathrm{~min}$ and resuspended at $3 \times 10^{6}$ cells $/ \mathrm{ml}$ in RPMI-1640 without serum and stimulated with either anti-CD3 (mouse monoclonal, clone OKT3, Sigma aldrich, USA) at the indicated concentrations, anti-CD3 with $3 \% \mathrm{H}_{2} \mathrm{O}_{2}$ (VWR, USA) and $3 \% \mathrm{H}_{2} \mathrm{O}_{2}$ for $2 \mathrm{~min}$ at $37^{\circ} \mathrm{C}$. To find out the optimal stimulation, cells were also stimulated with sodium orthovanadate (Sigma-Aldrich, Sweden) and sodium orthovanadate with $3 \% \mathrm{H}_{2} \mathrm{O}_{2}$ for $15 \mathrm{~min}$ at $37^{\circ} \mathrm{C}$. After stimulation, cells were immediately placed on ice, $1 \mathrm{ml}$ ice cold DPBS were added quickly before centrifugation at $300 \times \mathrm{g}$ for $5 \mathrm{~min}$. The pellets were lysed for $30 \mathrm{~min}$ at $4^{\circ} \mathrm{C}$ with $150 \mu \mathrm{l}$ lysis buffer (RIPA, Thermo Scientific, USA) including phosphatase inhibitor PhosSTOP (Sigma aldrich, USA) as well as protease inhibitor (Sigma aldrich, USA). Cellular debris were sedimented at $12000 \mathrm{xg}$ for $20 \mathrm{~min}$ at $4^{\circ} \mathrm{C}$ and supernatant harvested and stored at $-80^{\circ} \mathrm{C}$ for subsequent studies. Quantitation of proteins were performed with PierceTM BCA protein assay kit (Thermo Scientific, USA). SDS-PAGE and Western blotting were performed according to the manufacturer. Briefly, reduced SDS-PAGE was performed with Bio-Rad CriterionTM $12 \%$ respectively $7,5 \%$ TGX Stain-FreeTM Precast gels (Bio-Rad Laboratories, Hercules,CA) and proteins were transferred to $0,2 \mu \mathrm{m}$ PVDF (Bio Rad, USA) using Trans-blot Turbo transfer pack, mini format Trans blot Turbo Transfer system (Bio Rad, USA), Mini-PROTEAN TGX (Bio Rad, USA). Thereafter membranes were blocked with $0,1 \%$ TBS-T with $5 \%$ BSA for 60 min with agitation before incubation with Rabbit polyclonal antibodies to ZAP70 phospho Y493 (Abcam, Storbritannien), Rabbit monoclonal antibodies [EP2291Y] to ZAP70 phospho Y492 (Abcam, Storbritannien), and Anti-beta Actin antibodies (Abcam, Storbritannien. Antibody binding was detected with secondary antibodies Goat Anti-Rabbit IgG H\&L (HRP) (Abcam, Storbritannien) and HRP Anti-beta Actin antibody (Abcam, Storbritannien) and enhanced via Clarity MaxTM Western ECL chemiluminescence (ECL) detection kit (Amersham, Buckinghamshire, United Kingdom). The ChemiDoc Imaging systems (Bio Rad, USA) were used for imaging.

In-gel Proteolytic Digest. 25 mM NH${ }_{4} \mathrm{HCO}_{3}(100 \mathrm{mg} / 50$ $\mathrm{ml}), 25 \mathrm{mM} \mathrm{NH} \mathrm{HCO}_{3}$ in $50 \% \mathrm{MeCN}, 50 \% \mathrm{MeCN} / 5 \%$ formic acid, $12.5 \mathrm{ng} / \mu \mathrm{L}$ trypsin in $25 \mathrm{mM} \mathrm{NH}_{4} \mathrm{HCO}_{3}$ were firstly prepared. Acrylamide gel was cut between $50 \mathrm{kDa}$ to $75 \mathrm{kDA}$ - where ZAP-70 phosphoprotein were detected- and gel was sliced into small pieces and placed into siliconized tubes. Gels were covered with $500 \mu \mathrm{L}$ of $25 \mathrm{mM} \mathrm{NH}_{4} \mathrm{HCO}_{3}$ in $50 \% \mathrm{MeCN}$, vortexed for $15 \mathrm{~min}$. and the supernatant discarded. This protocol repeated twice. The gels were dried using Speed Vac. to complete dryness. The dried gels were then incubated with $200 \mu \mathrm{L}$ of $10 \mathrm{mM} \mathrm{DTT}$ in $25 \mathrm{mM} \mathrm{NH} 4 \mathrm{HCO} 3(1.5 \mathrm{mg} / \mathrm{mL})$ for $1 \mathrm{~h}$ at $56^{\circ} \mathrm{C}$. After reaction, the supernatant was removed and $200 \mu \mathrm{L}$ of $55 \mathrm{mM}$ iodoacetamide in $25 \mathrm{mM} \mathrm{NH}_{4} \mathrm{HCO}_{3}$ (10 $\mathrm{mg} / \mathrm{mL}$ ) was added. The reaction allowed proceeding in the dark for 45 minutes at room temperature. Then, the supernatant was removed and the fels were washed with $200 \mu \mathrm{L}$ of $25 \mathrm{mM} \mathrm{NH}_{4} \mathrm{HCO}_{3}$ and vortexed for $15 \mathrm{~min}$. This protocol was repeated three times and then the gels were dehydrated with $25 \mathrm{mM} \mathrm{NH}_{4} \mathrm{HCO}_{3}$ in $50 \% \mathrm{MeCN}$. The protocol was repeated twice and the gels were dried completely using Speed Vac. For digest, 100 uL of 12.5 
$\mathrm{ng} / \mu \mathrm{L}$ trypsin in $25 \mathrm{mM} \mathrm{NH}_{4} \mathrm{HCO}_{3}$ was added and the containers were placed on ice for 15 minutes for dehydration. Before incubation, $200 \mu \mathrm{L}$ of $25 \mathrm{mM}$ $\mathrm{NH}_{4} \mathrm{HCO}_{3}$ in $50 \% \mathrm{MeCN}$ was added and the samples were incubated at $37^{\circ} \mathrm{C}$ for $16 \mathrm{~h}$. After, the solution was transferred to clean tube and $100 \mathrm{uL}$ of $50 \% \mathrm{MeCN} / 5 \%$ formic acid was added into gel pieces and vortexed for 30 minutes and the extracted digested pooled together. This protocol was repeated for three times and the extracted protein digest samples was concentrated.

MALDI-TOF MS/MS Analysis. The residue was dissolved in $\mathrm{H}_{2} \mathrm{O}+0.1 \%$ TFA $(10 \mu \mathrm{L})$. The $1 \mu \mathrm{L}$ of matrix solution (DHB matrix was prepared by dissolving $25 \mathrm{mg}$ DHB in 1 $\mathrm{mL}$ of $50 \% \mathrm{MeCN}$ and $1 \%$ phosphoric acid $+0.1 \%$ TFA) and $1 \mu \mathrm{L}$ of sample was deposited together on the target plate. Mass-spectrometric analysis of the fractions was performed using a MALDI reflector time-of-flight mass spectrometer (Ultraflex mass spectrometer, BrukerDaltonics $\mathrm{GmbH}$, Bremen, Germany) equipped with a Scout-384 source in positive reflector mode unless otherwise stated. The spectra was collected by accumulating $\mathbf{5 0 0 0}$ laser shots under linear mode (relative laser focus: 80\%) and further analyzed with the Flexanalysis 3.0 software (Bruker Daltonics). The scanning was performed by using the RP Pepmix Par method, and the mass spectra were analyzed with flex Control software (Brucker Daltonic FLEXControl).

\section{REFERENCES}

1. Blackstock, W. P. \& Weir, M. P. Proteomics: Quantitative and physical mapping of cellular proteins. Trends in Biotechnology (1999).

2. Jensen, $\mathrm{O}$. N. Interpreting the protein language using proteomics. Nature Reviews Molecular Cell Biology (2006).

3. Reinders, J. \& Sickmann, A. State-of-the-art in phosphoproteomics. Proteomics (2005).

4. Ubersax, J. A. \& Ferrell, J. E. Mechanisms of specificity in protein phosphorylation. Nature Reviews Molecular Cell Biology (2007).

5. Morandell, S. et al. Phosphoproteomics strategies for the functional analysis of signal transduction. Proteomics (2006).

6. Thingholm, T. E., Jensen, O. N. \& Larsen, M. R. Analytical strategies for phosphoproteomics. Proteomics (2009).

7. Mann, M. et al. Analysis of protein phosphorylation using mass spectrometry: Deciphering the phosphoproteome. Trends in Biotechnology (2002).

8. Manning, G., Whyte, D. B., Martinez, R., Hunter, T. \& Sudarsanam, S. The protein kinase complement of the human genome. Science (2002).

9. Cohen, P. T. W. Protein phosphatase 1 - Targeted in many directions. J. Cell Sci. (2002).

10. Hunter, T. \& Sefton, B. M. Transforming gene product of Rous sarcoma virus phosphorylates tyrosine. Proc. Natl. Acad. Sci. U. S. A. (1980).

11. Graves, J. D. \& Krebs, E. G. Protein phosphorylation and signal transduction. in Pharmacology and Therapeutics (1999).
12. Harsha, H. C. \& Pandey, A. Phosphoproteomics in cancer. Molecular Oncology (2010).

13. Hunter, T. Signaling - 2000 and beyond. Cell (2000). 14. Sun, C. \& Bernards, R. Feedback and redundancy in receptor tyrosine kinase signaling: Relevance to cancer therapies. Trends in Biochemical Sciences (2014).

15. Yu, L. R., Issaq, H. J. \& Veenstra, T. D. Phosphoproteomics for the discovery of kinases as cancer biomarkers and drug targets. Proteomics - Clinical Applications (2007).

16. Sharma, K. et al. Ultradeep Human Phosphoproteome Reveals a Distinct Regulatory Nature of Tyr and Ser/ThrBased Signaling. Cell Rep. (2014).

17. Chan, A. C., Iwashima, M., Turck, C. W. \& Weiss, A. ZAP-70: A 70 kd protein-tyrosine kinase that associates with the TCR $\zeta$ chain. Cell (1992).

18. Chan, A. C., Irving, B. A., Fraser, J. D. \& Weiss, A. The $\zeta$ chain is associated with a tyrosine kinase and upon T-cell antigen receptor stimulation associates with ZAP-70, a 70-kDa tyrosine phosphoprotein. Proc. Natl. Acad. Sci. U. S. A. (1991).

19. Au-Yeung, B. B. et al. The structure, regulation, and function of ZAP-70. Immunol. Rev. (2009).

20. Sloan-Lancaster, J. et al. ZAP-70 association with T cell

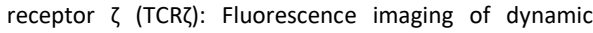
changes upon cellular stimulation. J. Cell Biol. (1998).

21. Au-Yeung, B. B., Shah, N. H., Shen, L. \& Weiss, A. ZAP70 in Signaling, Biology, and Disease. Annu. Rev. Immunol. (2018).

22. Jeffrey Chiang, Y. \& Hodes, R. J. Regulation of T cell development by c-Cbl: Essential role of Lck. Int. Immunol. (2015).

23. Irving, B. A., Chan, A. C. \& Weiss, A. Functional characterization of a signal transducing motif present in the T cell antigen receptor $\zeta$ chain. J. Exp. Med. (1993).

24. Straus, D. B. \& Weiss, A. The CD3 chains of the T cell antigen receptor associate with the ZAP-70 tyrosine kinase and are tyrosine phosphorylated after receptor stimulation. J. Exp. Med. (1993).

25. Osman, N., Lucas, S. \& Cantrell, D. The role of tyrosine phosphorylation in the interaction of cellular tyrosine kinases with the $T$ cell receptor $\zeta$ chain tyrosine-based activation motif. Eur. J. Immunol. (1995).

26. Irving, B. A. \& Weiss, A. The cytoplasmic domain of the $T$ cell receptor $\zeta$ chain is sufficient to couple to receptorassociated signal transduction pathways. Cell (1991).

27. Reth, M. Antigen receptor tail clue [5]. Nature (1989). 28. Sunder-Plassmann, R., Lialios, P., Madsen, M., Koyasu, S. \& Reinherz, E. L. Eur. J. Immunol. (1997).

29. Chakraborty, A. K. \& Weiss, A. Insights into the initiation of TCR signaling. Nature Immunology (2014).

30. Iwashima, M., Irving, B. A., Van Oers, N. S. C., Chan, A. C. \& Weiss, A. Sequential interactions of the TCR with two distinct cytoplasmic tyrosine kinases. J. Immunol. (2014). 31. Neumeister, E. N. et al. Mol. Cell. Biol. (1995).

32. Di Bartolo, V. et al. Tyrosine 319, a newly identified phosphorylation site of ZAP-70, plays a critical role in T cell antigen receptor signaling. J. Biol. Chem. (1999).

33. Klammt, C. et al. T cell receptor dwell times control the kinase activity of Zap70. Nat. Immunol. (2015).

34. Visco, C. et al. Activation of Zap-70 tyro sine kinase due to a structural rearrangement induced by tyrosine 
phosphorylation and/or ITAM binding. Biochemistry (2000).

35. Chan, A. C. et al. Activation of ZAP-70 kinase activity by phosphorylation of tyrosine 493 is required for lymphocyte antigen receptor function. EMBO J. (1995).

36. Liu, H., Purbhoo, M. A., Davis, D. M. \& Rudd, C. E. SH2 domain containing leukocyte phosphoprotein of 76-kDa (SLP-76) feedback regulation of ZAP-70 microclustering. Proc. Natl. Acad. Sci. U. S. A. (2010).

37. Wardenburg, J. B. et al. Phosphorylation of SLP-76 by the ZAP-70 protein-tyrosine kinase is required for T-cell receptor function. J. Biol. Chem. (1996).

38. Bogin, Y., Ainey, C., Beach, D. \& Yablonski, D. SLP-76 mediates and maintains activation of the Tec family kinase ITK via the T cell antigen receptor-induced association between SLP-76 and ITK. Proc. Natl. Acad. Sci. U. S. A. (2007).

39. Ji, Q., Ding, Y. \& Salomon, A. R. SRC homology 2 domain-containing leukocyte phosphoprotein of $76 \mathrm{kDa}$ (SLP-76) N-terminal tyrosine residues regulate a dynamic signaling equilibrium involving feedback of proximal T-cell receptor (TCR) signaling. Mol. Cell. Proteomics (2015).

40. Wang, H. et al. ZAP-70: an essential kinase in T-cell signaling. Cold Spring Harbor perspectives in biology (2010).

41. Yan, Q. et al. Structural Basis for Activation of ZAP-70 by Phosphorylation of the SH2-Kinase Linker. Mol. Cell. Biol. (2013)

42. Sup, S. J. et al. ZAP-70 expression in B-cell hematologic malignancy is not limited to CLL/SLL. Am. J. Clin. Pathol. (2004).

43. Rodríguez-Vicente, A. E., Díaz, M. G. \& HernándezRivas, J. M. Chronic lymphocytic leukemia: A clinical and molecular heterogenous disease. Cancer Genetics (2013). 44. Shim, J. H. et al. (-)-Epigallocatechin gallate regulates CD3-mediated $T$ cell receptor signaling in leukemia through the inhibition of ZAP-70 kinase. J. Biol. Chem. (2008).

45. Fujimoto, M. B Cells. in Immunology of the Skin: Basic and Clinical Sciences in Skin Immune Responses (2016).

46. Richardson, S. J. et al. ZAP-70 expression is associated with enhanced ability to respond to migratory and survival signals in B-cell chronic lymphocytic leukemia (BCLL). Blood (2006).

47. Rassenti, L. Z. et al. ZAP-70 compared with immunoglobulin heavy-chain gene mutation status as a predictor of disease progression in chronic lymphocytic leukemia. N. Engl. J. Med. (2004).

48. Wiestner, A. et al. ZAP-70 expression identifies a chronic lymphocytic leukemia subtype with unmutated immunoglobulin genes, inferior clinical outcome, and distinct gene expression profile. Blood (2003).

49. Orchard, J. A. et al. ZAP-70 expression and prognosis in chronic lymphocytic leukaemia. Lancet (2004).

50. Boersema, P. J. et al. In-depth qualitative and quantitative profiling of tyrosine phosphorylation using a combination of phosphopeptide immunoaffinity purification and stable isotope dimethyl labeling. Mol. Cell. Proteomics (2010).

51. Mosbach, K. \& Ramström, O. The emerging technique of molecular imprinting and its future impacton biotechnology. Bio/Technology (1996).
52. Wulff, G. Molecular Imprinting in Cross-Linked Materials with the Aid of Molecular Templates- A Way towards Artificial Antibodies. Angewandte Chemie International Edition in English (1995).

53. Haginaka, J., Tabo, H. \& Matsunaga, H. Preparation of molecularly imprinted polymers for organophosphates and their application to the recognition of organophosphorus compounds and phosphopeptides. Anal. Chim. Acta (2012).

54. Emgenbroich, M. et al. A phosphotyrosine-imprinted polymer receptor for the recognition of tyrosine phosphorylated peptides. Chem. - A Eur. J. (2008).

55. Chen, J. et al. Validation of molecularly imprinted polymers for side chain selective phosphopeptide enrichment. J. Chromatogr. A (2016).

56. Helling, S. et al. Ultratrace enrichment of tyrosine phosphorylated peptides on an imprinted polymer. Anal. Chem. (2011).

57. Hall, A. J. et al. Urea host monomers for stoichiometric molecular imprinting of oxyanions. J. Org. Chem. (2005). 58. Shinde, S.; Incel, A.; et al. J. Am. Chem. Soc. (2020).

59. Incel A. et al. Analytical Chemistry (2021).

60. Hargrove, A. E.; Nieto, S.; Zhang, T.; Sessler, J. L.; Anslyn, E. V. Artificial Receptors for the Recognition of Phosphorylated Molecules. Chem. Rev. 2011, 111 (11), 6603-6782.

61. Ravikumar, I.; Ghosh, P. Recognition and separation of sulfate anions. Chem. Soc. Rev. 2012, 41 (8), 3077-3098.

62. Rosenwald, A. et al. Relation of gene expression phenotype to immunoglobulin mutation genotype in B cell chronic lymphocytic leukemia. J Exp Med. 2001;194: 1639-1648.

63. Tara L. Lysechko and Hanne L. Ostergaard, J Immunol 2005; 174:7807-7814.

64. Natarajan V, Vepa S, Verma RS, Scribner WM. Role of protein tyrosine phosphorylation in $\mathrm{H} 2 \mathrm{O} 2$-induced activation of endothelial cell phospholipase D. Am J Physiol. 1996 Sep;271(3 Pt 1):L400-8.

65 . Sylvain Tessier, Alcide Chapdelaine, Simone Chevalier. Molecular and Cellular Endocrinology, 64 (1989) 87-94.

66. Huber RG, Fan H, Bond PJ (2015) The Structural Basis for Activation and Inhibition of ZAP-70 Kinase Domain. PLoS Comput Biol 11(10): e1004560

67. Haopeng Wang, Theresa A. Kadlecek, Byron B. AuYeung, Hanna E. Sjölin Goodfellow, Lih-Yun Hsu, Tanya S. Freedman, and Arthur Weiss. Cold Spring Harb Perspect Biol. 2010 May; 2(5): a002279.

68. Andrew C. Chan, Mark Dalton, Robin Johnson, Guanghui Kong, Tian Wang, Rich Thoma, Tomohiro Kurosaki. The EMBO Journal vol.14 no.11 pp.2499-2508, 1995.

69. Tomas Brdicka, Theresa A. Kadlecek, Jeroen P. Roose, Alexander W. Pastuszak, Arthur Weiss. Molecular and Cellular Biology May 2005, 25 (12) 4924-4933

70. Huber RG, Fan H, Bond PJ. The Structural Basis for Activation and Inhibition of ZAP-70 Kinase Domain. PLoS Comput Biol. (2015)

71. Mutation of Tyrosines 492/493 in the Kinase Domain of ZAP-70 Affects Multiple T-cell Receptor Signaling Pathways. Mège, Dominique et al. Journal of Biological Chemistry, Volume 271, Issue 51, 32644 - 32652. 


\section{Supporting Information}

\section{MIP-Binders for Sequence Specific Phosphopeptide Capture of ZAP-70 regulatory motifs}

Anıl Incel ${ }^{1}$, Sudhirkumar Shinde ${ }^{1}$, Ignacio A. Diez ${ }^{2}$, Alper Yılmaz ${ }^{1}$, Thomas Sjoberg ${ }^{1}$, Maria M. Stollenwerk ${ }^{1}$, Ole N. Jensen ${ }^{2, *}$ and Börje Sellergren ${ }^{1, *}$

${ }^{1}$ Department of Biomedical Science, Faculty of Health and Society, Malmö University, 20506 Malmö, Sweden

${ }^{2}$ Department of Biochemistry and Molecular Biology and VILLUM Center for Bioanalytical Sciences, University of Southern Denmark, DK-5230 Odense M, Denmark

\section{Materials}

Pentaerythritol triacrylate (PETA) was from Polysciences (Warrington, PA, USA). N,NO -azo-bis(2,4dimethyl)valeronitrile (ABDV) was from Wako Chemicals $\mathrm{GmbH}$ (Neuss, Germany); 1-hydroxybenzotriazole (HOBt), 1,2,2,6,6-pentamethylpiperidine (PMP), triethylammonium bicarbonate (TEAB) buffer (1 M), acetic anhydride (Ac2O) and formic acid (FA) were from Fluka (Deisenhofen, Germany). Trifuoroacetic acid (TFA), acetonitrile $(A C N)$, methanol $(\mathrm{MeOH})$ were from VWR chemicals. Dry dichloromethane (dry DCM), triethylamine (TEA), ammonium hydrogen difuoride (NH4HF2), 2,5-dihydroxybenzoic acid (DHB), piperidine, ninhydrin, acrylamide, iodoacetamide (IAA) and 1,4-dithiothreitol (DTT) were from Sigma-Aldrich (Milwaukee, USA). Dimethylformamide (DMF), dry acetonitrile (dry ACN) were from Acros Organics. Fmoc-pTyrOH, Fmoc-pSer-OH, Fmoc-Tyr-OH and Fmoc-Gly-OH were from Bachem GmbH (Weil am Rhein, Germany). FmocTyr(PO(NMe2)2)-OH, $\mathrm{N}$-ethyldiisopropylamine (DIPEA), (benzotriazol-1-yloxy)tripyrrolidinophosphonium hexafuorophosphate (PyBOP) and dichloromethane (DCM) were from Merck KGaA (Dramstadt, Germany). Empore C8 extraction disk was from 3M Bioanalytical Technologies (St. Paul, MN, USA). Trypsin was from Promega (Madison, WI, USA). Proteins and peptides. The twelve proteins used for protein digestion were: carbonic anhydrase (bovine), ribonuclease B (bovine pancreas), serum albumin (bovine), lactoglobulin (bovine), $\alpha$-casein (bovine), $\beta$-casein (bovine), ovalbumin (chicken), lysozyme (chicken), alcohol dehydrogenase (Baker's yeast), myoglobin (whale skeletal muscle), $\alpha$-amylase (Bacillus species) and transferrin (human). Phosphopeptides GADDSpYpYTAR, GADDSpYYTAR, GADDSYpYTAR, GADDSYYTAR were custom synthesized by LifeTein LLC. 


\section{Synthesis of Templates}

Esterification of amino acids on 2-chloro trithyl chloride followed by deprotection
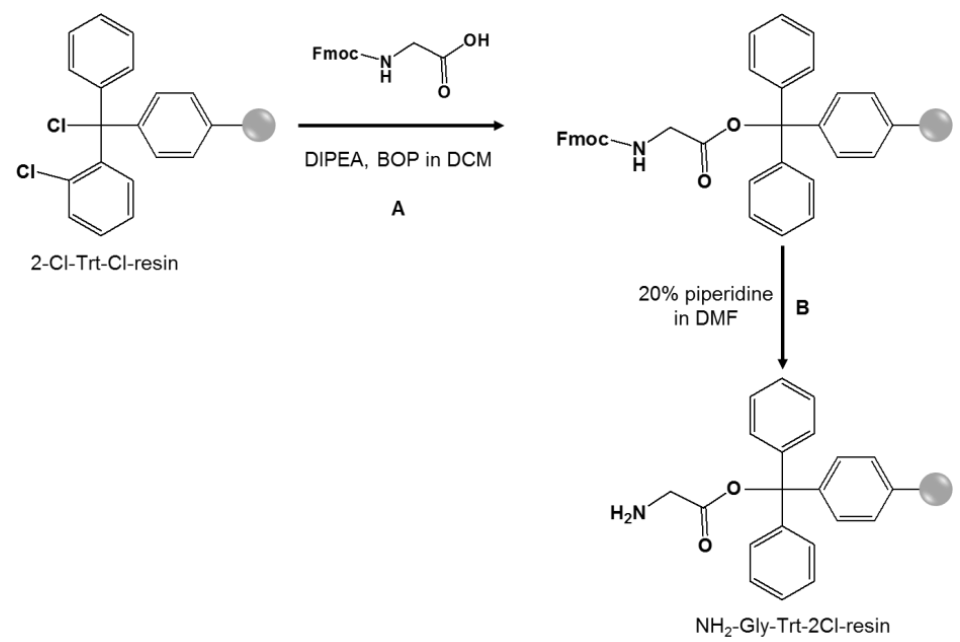

Esterification of resin by Fmoc-Gly-OH (A) and then deprotection of Fmoc- group (B)

The esterification of aminoacids on solid support was conducted in followed protocol, $5 \mathrm{~g}$ of 2-chloro trityl chloride (1.55 mmol of $\mathrm{Cl}$ per $\mathrm{g}$ of resin) was added in a $50 \mathrm{~mL}$ capacity solid cartridge. Then followingly, FmocGly-OH (4.62 g) and $40 \mathrm{~mL} \mathrm{DCM}$ were added. DIPEA $(6.8 \mathrm{~mL})$ was added in the mixture. The resultant solution was shaked for $1 \mathrm{~h}$. After shaking, the solvent was removed, the resin was washed with DCM ( $3 \times 20 \mathrm{~mL})$, then DCM:MeOH:DIPEA (80:15:5) once, and it is filtered after shaking for $10 \mathrm{~min}$. The resin was consecutively washed by $\mathrm{DCM}(3 \times 20 \mathrm{~mL}), \mathrm{MeOH}(3 \times 20 \mathrm{~mL})$, and $\mathrm{Et}_{2} \mathrm{O}(3 \times 20 \mathrm{~mL})$. After excess washing, the resin was dried in the vacuum oven for $1 \mathrm{~h}$ and then Fmoc quantification was completed as explained follow: Fmoc-Gly-2Cl trityl resin ( $5 \mathrm{mg}$ ) was taken in $20 \mathrm{~mL}$ volumetric flask and piperidine ( $2 \mathrm{~mL}$ of $20 \% \mathrm{v} / \mathrm{v}$ in DMF) was added. The solution was shaken for 1 hour and then diluted by using $\mathrm{MeOH}$. The solution absorbance was measured at $313.0 \mathrm{~nm}$ for the determination of Fmoc quantitatively. The loading vale ( $f$ ) as mmol/g was calculated by using equation 1 and 2 .

$$
\text { Equation 1: } \quad f^{\prime}=\frac{1000 * f}{1000-(f * M w \text { added })} \quad \text { Equation 2: } \quad f=\frac{1.28 * A * 2}{\# m g \text { of resin }}
$$

The quantification of Fmoc- group was completed by using two masses and their corresponding absorbance value. Fmoc- amounts were calculated as 0.727 and $0.789 \mathrm{mmol} / \mathrm{g}$ for $6.14 \mathrm{mg}$ of resin $\left(\lambda_{313}=1.744\right)$ and for 6.79 $\mathrm{mg}$ of resin $\left(\lambda_{313}=2.094\right)$, respectively. The average of Fmoc amount/g of resin was found as $0.75 \mathrm{mmol} / \mathrm{g}$. The esterification of aminoacids and coupling reaction between Fmoc-Gly-OH and resin was confirmed after the quantification of Fmoc-protecting group by spectroscopic method. Therefore, the Fmoc de-protection was carried out washing the resin using piperidine ( $40 \mathrm{~mL}$ of $20 \% \mathrm{v} / \mathrm{v}$ in DMF) for shaking $8 \mathrm{~min}$. This step was repeated twice and the consecutive washing step was done by using DCM ( $3 \times 20 \mathrm{~mL}), \mathrm{MeOH}(3 \times 20 \mathrm{~mL})$, and $\mathrm{Et}_{2} \mathrm{O}(3 \times 20 \mathrm{~mL})$. After washing, the resin was vacuum dried overnight. 


\section{Coupling of Fmoc-Tyr(t-Bu)-OH on $\mathrm{NH}_{2}$-Gly-2Cl-Trt-resin}

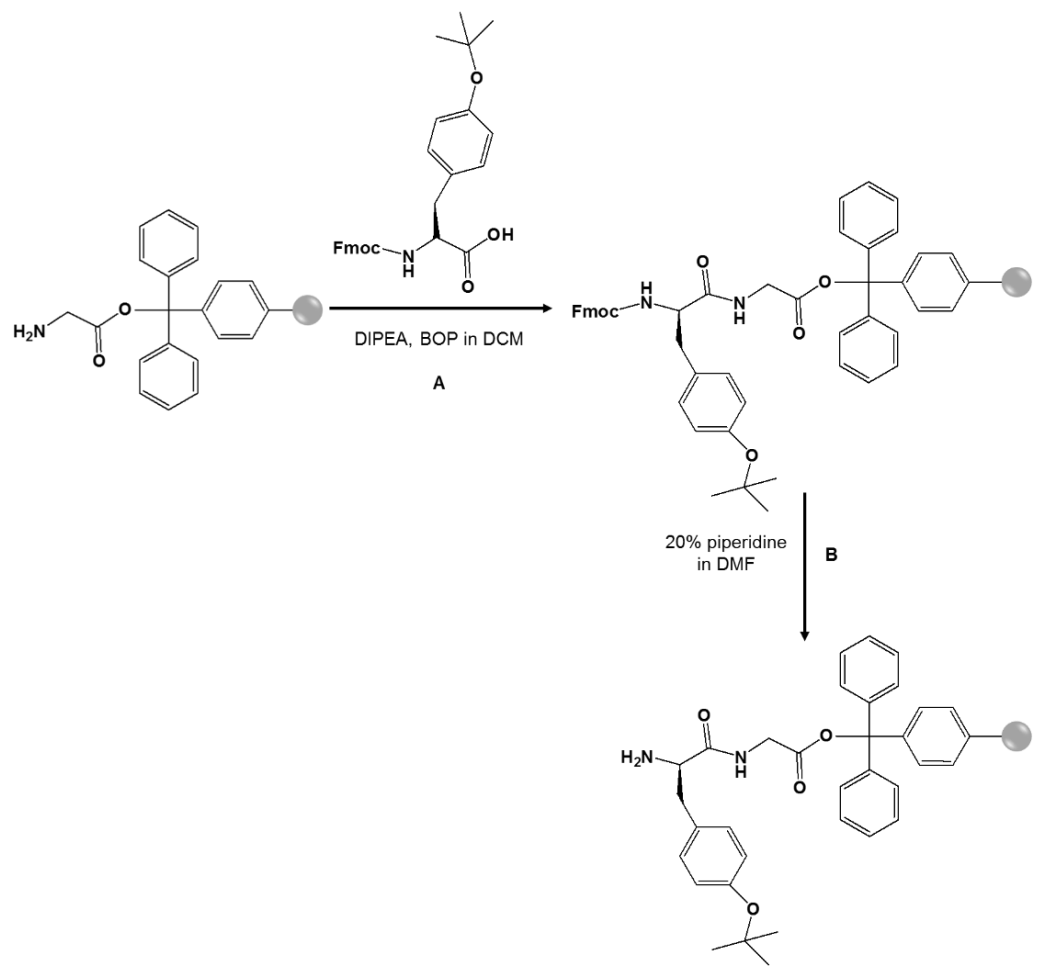

Coupling of Fmoc-Tyr(t-Bu)-OH on $\mathrm{NH}_{2}$-Gly-2Cl-Trt-resin (A) and deprotection of Fmoc- group (B)

In the coupling of Fmoc-Tyr(t-Bu)-OH on $\mathrm{NH}_{2}$-Gly-2Cl-Trt-resin protocol, $1 \mathrm{~g}$ of NH $\mathrm{N}_{2}-\mathrm{Tyr}(\mathrm{t}-\mathrm{Bu})$-Gly-OH loaded resin was taken in a $10 \mathrm{~mL}$ capacity solid phase cartridge and Fmoc-Tyr(t-Bu)-OH (1.38 g) was added. Then, BOP (1.32 $\mathrm{g})$, HOBT $(0.43 \mathrm{~g})$ and DMF $(6 \mathrm{~mL})$ were following added. DIPEA $(1.1 \mathrm{~mL})$ was finally added in the resultant mixture. The reaction was completed after $4 \mathrm{~h}$, then the solvent was removed, and the resin was washed using DCM ( $3 \mathrm{x}$ $15 \mathrm{~mL}), \mathrm{MeOH}(3 \times 15 \mathrm{~mL})$, and $\mathrm{Et}_{2} \mathrm{O}(3 \times 15 \mathrm{~mL})$, consecutively. After washing, the resin was dried and kept at $4^{\circ} \mathrm{C}$. The deprotection of Fmoc was conducted the same manner as it was explained in the previous section. 

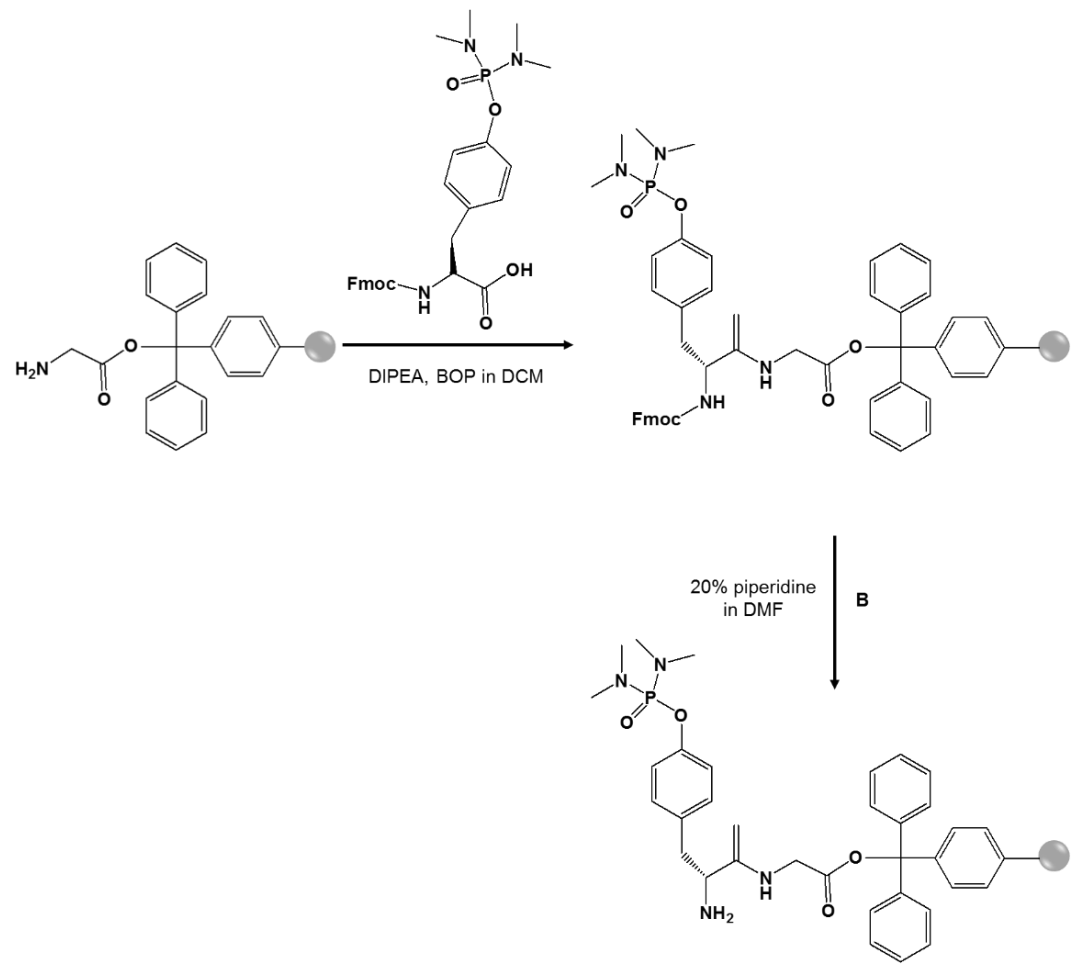

Coupling of Fmoc- $\mathrm{Tyr}\left(\mathrm{PO}_{3}\left(\mathrm{NMe}_{2}\right)_{2}\right)-\mathrm{OH}$ on $\mathrm{NH}_{2}$-Gly-2Cl-Trt-resin (A) and then deprotection of Fmoc- group (B)

In the coupling of Fmoc- $\mathrm{Tyr}\left(\mathrm{PO}_{3}\left(\mathrm{NMe}_{2}\right)_{2}\right)-\mathrm{OH}$ on $\mathrm{NH}_{2}-\mathrm{Gly}-2 \mathrm{Cl}-\mathrm{Trt}-\mathrm{resin}$ protocol, $2 \mathrm{~g}$ of NH $\mathrm{NH}_{2}-\mathrm{Tyr}(\mathrm{t}-\mathrm{Bu})-\mathrm{Gly}-\mathrm{OH}$ loaded resin was taken in a $25 \mathrm{~mL}$ capacity solid phase cartridge and $\mathrm{Fmoc}-\mathrm{Tyr}\left(\mathrm{PO}_{3}\left(\mathrm{NMe}_{2}\right)_{2}\right)-\mathrm{OH}(1.24 \mathrm{~g})$ was added. Then, BOP $(1.21 \mathrm{~g})$, HOBT $(0.365 \mathrm{~g})$ and DMF $(15 \mathrm{~mL})$ were following added. DIPEA $(1 \mathrm{~mL})$ was finally added in the resultant mixture. The reaction was completed after $4 \mathrm{~h}$, then the solvent was removed, and the resin was washed using DCM $(3 \times 15 \mathrm{~mL}), \mathrm{MeOH}(3 \times 15 \mathrm{~mL})$, and $\mathrm{Et}_{2} \mathrm{O}(3 \times 15 \mathrm{~mL})$, consecutively. After washing, the resin was dried and kept at $4^{\circ} \mathrm{C}$. The deprotection of Fmoc was conducted the same manner as it was explained in the previous section. 
Coupling of building blocks on resins for the formation of tripeptide structures

The coupling of non-phosphorylated and phosphorylated building blocks, which are Fmoc-Tyr(t-Bu)-OH and Fmoc- $\mathrm{Tyr}\left(\mathrm{PO}_{3} \mathrm{~N}_{2} \mathrm{Me}_{4}\right)-\mathrm{OH}$ on $\mathrm{NH}_{2}-\mathrm{Tyr}(\mathrm{t}-\mathrm{Bu})$-Gly-trityl-resin and $\mathrm{NH}_{2}-\mathrm{Tyr}\left(\mathrm{PO}_{3} \mathrm{NMe}_{2}\right)_{2}-\mathrm{Gly}$-trityl-resin was shown in Table S1.

\begin{tabular}{|c|c|c|c|c|c|c|c|c|}
\hline \multirow[t]{4}{*}{ Epitope } & Fmoc- & Fmoc- & $\mathrm{NH}_{2-}^{-}$ & $\mathrm{NH}_{2^{-}}$ & $B O P$ & HOBT & $D M F$ & DIPEA \\
\hline & $\operatorname{Tyr}(t-B u)-$ & $\operatorname{Tyr}\left(\mathrm{PO}_{3} \mathrm{~N}_{2} \mathrm{Me}_{4}\right)-$ & $G l y(t-B u)-$ & $\mathrm{Tyr}\left(\mathrm{PO}_{3} \mathrm{~N}_{2} \mathrm{Me}_{4}\right)-$ & (g) & (g) & $(m L)$ & $(m L)$ \\
\hline & $\mathrm{OH}(g)$ & $\mathrm{OH}(g)$ & $\mathrm{OH}$ & $\mathrm{OH}$ & & & & \\
\hline & & & resin $(g)$ & $\operatorname{Resin}(g)$ & & & & \\
\hline$Y Y$ & 1.38 & - & 1.0 & - & 1.32 & 0.43 & 6 & 1.1 \\
\hline$Y p Y$ & 1.38 & - & - & 1.0 & 1.36 & 0.43 & 6 & 1.04 \\
\hline$p Y Y$ & - & 0.73 & 1.0 & - & 0.60 & 0.19 & 6 & 0.49 \\
\hline$p Y p Y$ & - & 0.73 & - & 1.0 & 0.60 & 0.19 & 6 & 0.49 \\
\hline
\end{tabular}

The synthesis was followed by the same manner as it was explained in the first coupling step by omitting deprotection of Fmoc group. Each synthesis was completed by using $10 \mathrm{~mL}$ capacity solid phase cartridges and each resin was washed using DCM $(3 \times 20 \mathrm{~mL}), \mathrm{MeOH}(3 \times 15 \mathrm{~mL})$, and $\mathrm{Et}_{2} \mathrm{O}(3 \times 15 \mathrm{~mL})$ before dried and kept at $4^{\circ} \mathrm{C}$. The coupling for the preparation of each tripeptide sequences were shown below for the preparation of $\mathrm{YY}$, YpY, pYY, and pYpY templates.

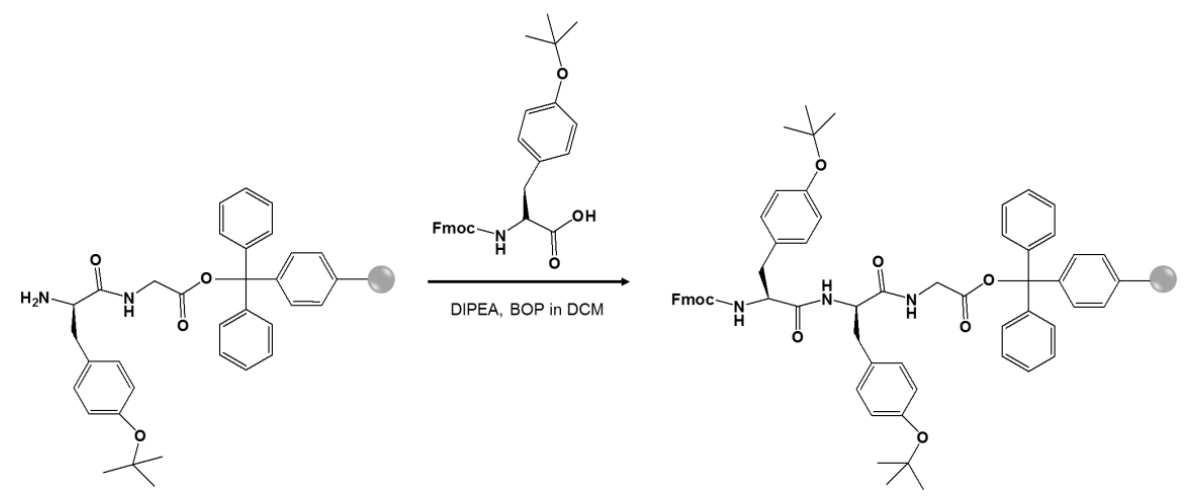

Coupling of Fmoc-Tyr(t-Bu)-OH on $\mathrm{NH}_{2}$-Tyr(t-Bu)-Gly-trityl-resin for stable template (YY template) preparation.

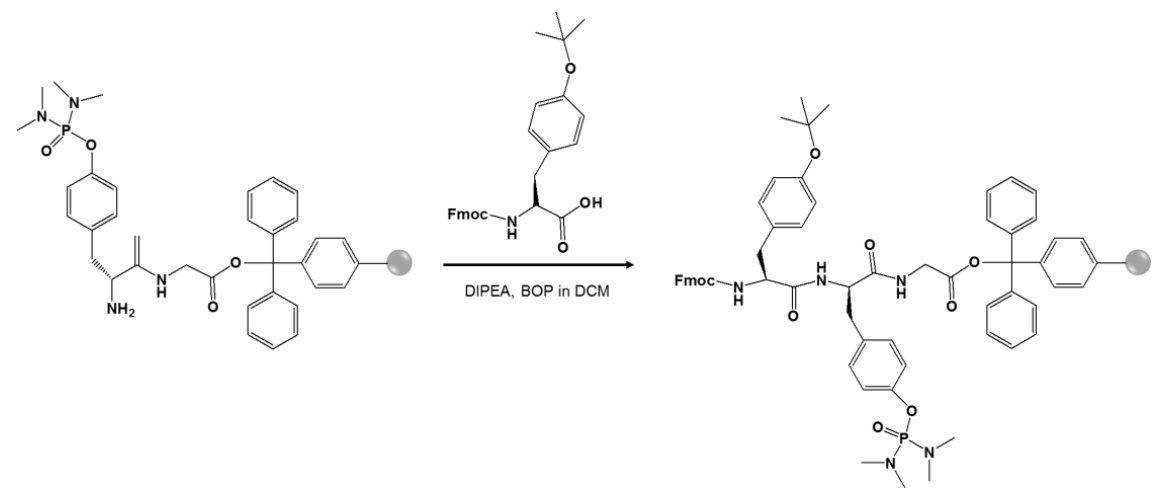

Coupling of Fmoc-Tyr(t-Bu)-OH on $\mathrm{NH}_{2}-\mathrm{Tyr}\left(\mathrm{PO}_{3} \mathrm{NMe}_{2}\right)_{2}$-Gly-trityl-resin for stable template ( $\mathrm{YpY}$ template) preparation 

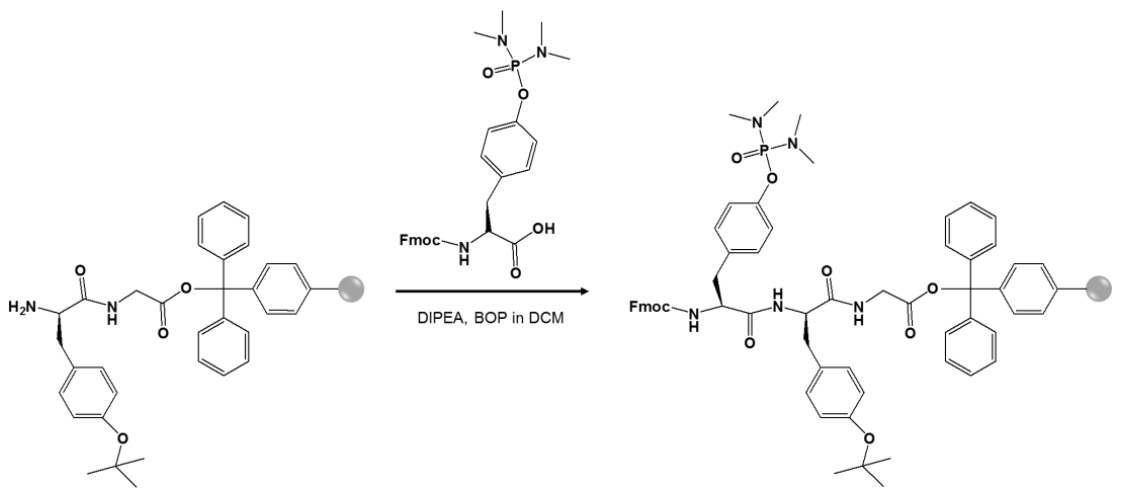

Coupling of Fmoc- $\mathrm{Tyr}\left(\mathrm{PO}_{3} \mathrm{~N}_{2} \mathrm{Me}_{4}\right)-\mathrm{OH}$ on $\mathrm{NH}_{2}$-Tyr(t-Bu)-Gly-trityl-resin for stable template ( $\mathrm{pYY}$ template) preparation

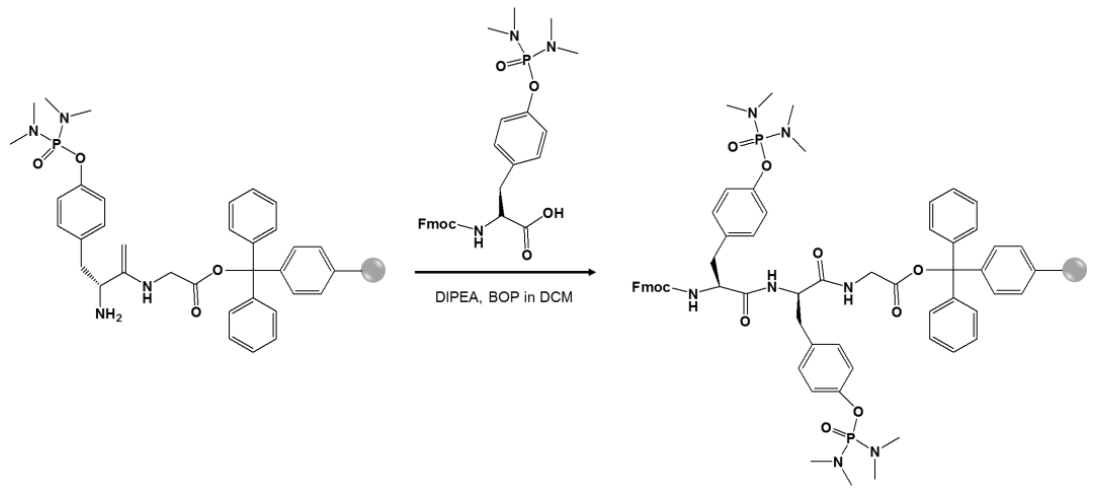

Coupling of Fmoc- $\mathrm{Tyr}\left(\mathrm{PO}_{3} \mathrm{~N}_{2} \mathrm{Me}_{4}\right)-\mathrm{OH}$ on $\mathrm{NH}_{2}-\mathrm{Tyr}\left(\mathrm{PO}_{3} \mathrm{NMe}_{2}\right)_{2}$-Gly-trityl-resin for stable template ( $\mathrm{pYpY}$ template) preparation 
Clevage of peptides from solid support, methylation on C-terminus, and deprotection of 0 -[bisdimethylamino] group of phosphonoltyrosine-containing peptides
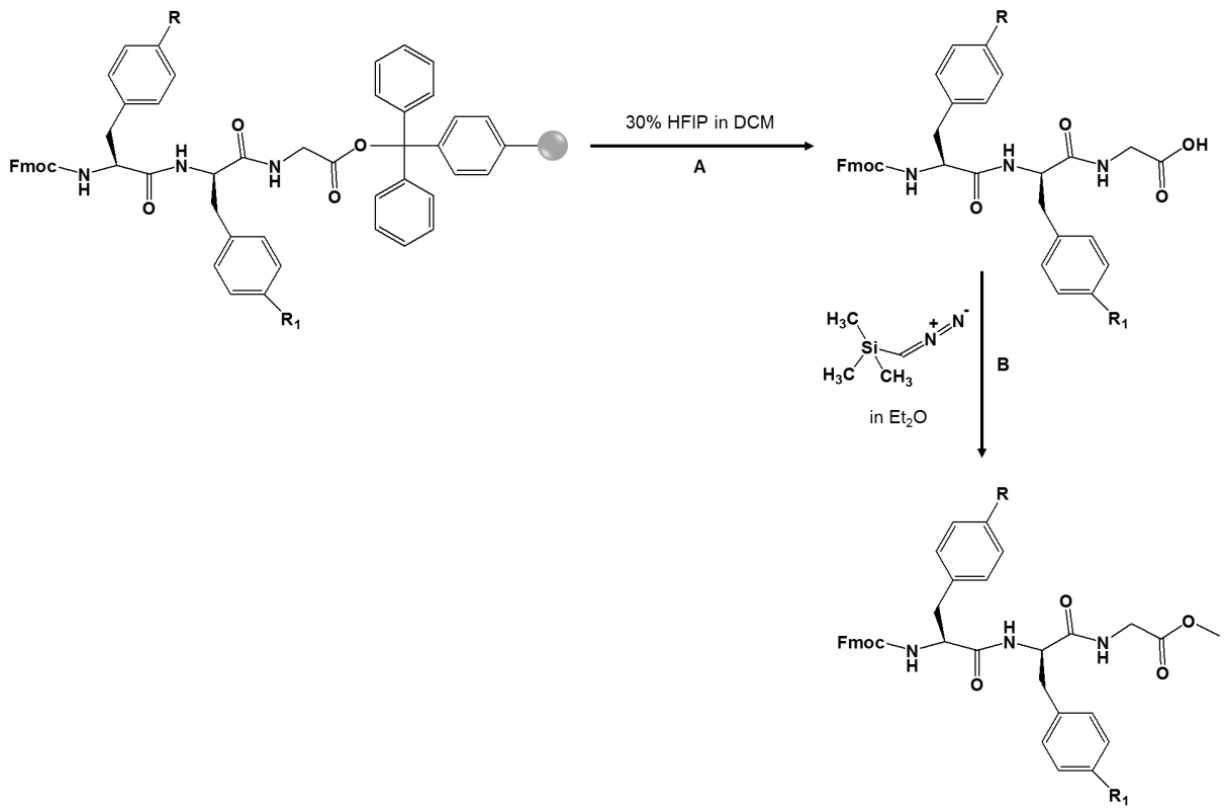

Cleavage of solid support resin from synthesized peptides (A) and methylation on C-terminus (B)

Each peptide-resin complex was incubated using $10 \mathrm{~mL}$ of $30 \%$ HFIP prepared in DCM for $1 \mathrm{~h}$ and each resin was washed with DCM $(2 \times 10 \mathrm{~mL})$ after filtration and evaporation of solvent. Then, each cleaved peptide was dissolved in $\mathrm{MeOH}: \mathrm{DCM}(1: 10)$ and (trimethylsilyl)diazomethane (2.0 M prepared in $\mathrm{Et}_{2} \mathrm{O}$ ) was added dropwise in the peptide solution under flowing of $\mathrm{N} 2$ gas for methylation. Moreover, each peptide was added in $1 \mathrm{~mL}$ of TFA:H2O (9:1) for deprotection of O-[bis-dimethylamino] group. The cleavage of peptide, methylation and deprotection were monitored by bromophenol blue (BPB) test and confirmed by mass analysis (Table S2).

\begin{tabular}{|c|c|c|c|}
\hline Tripeptide Structure & After Cleavage & After Methylation on C terminus & After deprotection \\
\hline$Y Y: F m o c-Y-Y-G-O C H_{3}$ & 736.4 & 750.3 & 638.3 \\
\hline $\mathrm{YpY}: \mathrm{Fmoc}-\mathrm{Y}-\mathrm{Y}\left(\mathrm{OPO}_{3} \mathrm{H}_{2}\right)-\mathrm{G}-\mathrm{OCH}_{3}$ & 814.4 & 828.4 & 718.5 \\
\hline pYY: Fmoc- $Y\left(\mathrm{OPO}_{3} \mathrm{H}_{2}\right)-\mathrm{Y}-\mathrm{G}-\mathrm{OCH}_{3}$ & 814.3 & 828.2 & 718.4 \\
\hline pYpY: Fmoc- $Y\left(\mathrm{OPO}_{3} \mathrm{H}_{2}\right)-\mathrm{Y}\left(\mathrm{OPO}_{3} \mathrm{H}_{2}\right)-\mathrm{G}-\mathrm{OCH}_{3}$ & 892.3 & 906.4 & 798.0 \\
\hline
\end{tabular}


The synthesized templates were characterized by mass analysis and the spectrum were shown in Figure S1.
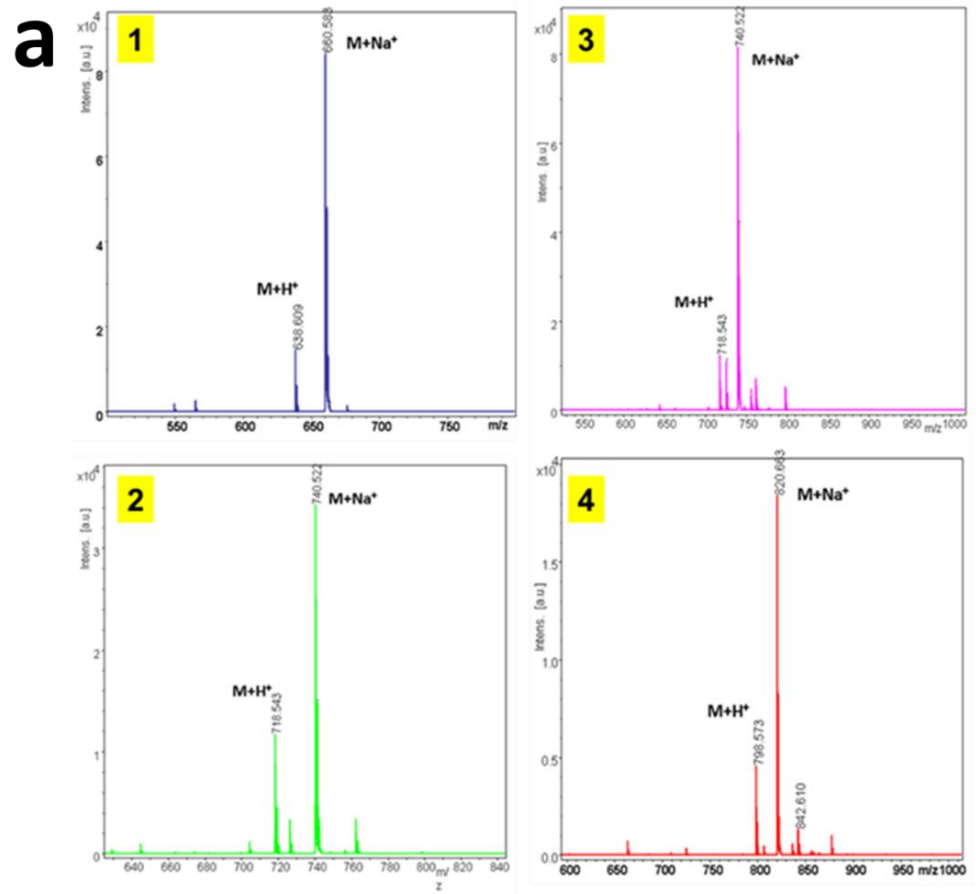

b

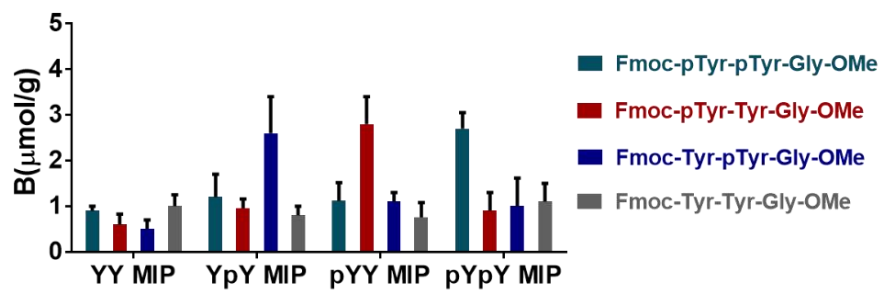

Figure S1: The mass analysis of synthesized templates after deprotection of $O$-[bis-dimethylamino] group and template binding. a Fmoc-Tyr-Tyr-Gly-OMe (1), Fmoc-Tyr-pTyr-Gly-OMe (2), Fmoc-pTyr-Tyr-Gly-OMe (3) and Fmoc-pTyr-pTyr-GlyOMe (4). The masses of peptide are $\mathrm{M}^{+} \mathrm{H}^{+}$and $\mathrm{M}^{+} \mathrm{Na}^{+}$. $\mathbf{b}$ Template binding test.

Finally, each template was dissolved in $\mathrm{H}_{2} \mathrm{O}: \mathrm{t}-\mathrm{BuOH}(1: 1)$ and $\mathrm{MeCN}: \mathrm{H}_{2} \mathrm{O}$ (1:1) and the white powder from each was obtained after lyophilization. They are kept in the freezer to be used further experiment. YY, YpY, pYY, and pYpY MIPs were tested with the mixture of $\mathrm{N}$ - and C- protected templates prepared in $90 \% \mathrm{MeCN}+0.1 \%$ TFA. The binding results in Figure $1 \mathrm{~b}$ confirms the selectivity of sequence specific binders for the corresponding target peptides. 


\section{Preparation of Protein Digestion}

12 protein digest was prepared by using following twelve proteins, which are carbonic anhydrase, bovine serum albumin (BSA), ovalbumin, $\alpha$-casein, $\beta$-casein, $\beta$-lactoglobulin, RNaseB, alcohol dehydrogenase, myglobulin, transferrin, lysozyme, and alpha amylase. Each protein was prepared in $50 \mathrm{mM}$ of triethylammonium bicarbonate (TEAB) buffer to achieve the final concentration of $50 \mathrm{pmol} / \mu \mathrm{L}(50 \mu \mathrm{M})$. The protein solution (100 $\mu \mathrm{L}$ ) of each was mixed with each other and the total volume and mass of mixture was identified as $1200 \mu \mathrm{L}$ and $2286.3 \mu \mathrm{g}$, respectively. The mixture was reduced by adding $12.1 \mu \mathrm{L}$ of $1 \mathrm{M} \mathrm{DTT}$ and incubated at $56^{\circ} \mathrm{C}$ for $1 \mathrm{~h}$. In next step, the mixture was alkylated by adding $24.7 \mu \mathrm{L}$ of $1 \mathrm{M}$ of IAA and incubated at room temperature in dark for $45 \mathrm{~min}$. The alkylation process was stopped by adding $12.5 \mu \mathrm{L}$ of $1 \mathrm{M}$ DTT. In the last three steps that it was carried out, the total volume of mixture was increased to $1249.3 \mu \mathrm{L}$ and the total protein concentration was decreased to $48.0 \mathrm{pmol} / \mu \mathrm{L}$. From the total mixture solution. $82 \mu \mathrm{L}(150 \mu \mathrm{g})$ was taken and it was digested overnight by using $2 \%(\mathrm{w} / \mathrm{w})$ Promega trypsin. The digested solution was diluted with $0.1 \%$ TFA to achieve the final concentration until decreased to $1 \mathrm{pmol} / \mu \mathrm{L}$ and each aliquot was prepared as $10 \mu \mathrm{L}$ and they were stored in the trypsin drawer at $-20^{\circ} \mathrm{C}$ freezer.

\section{Batch binding of decapeptides using imprinted polymer}

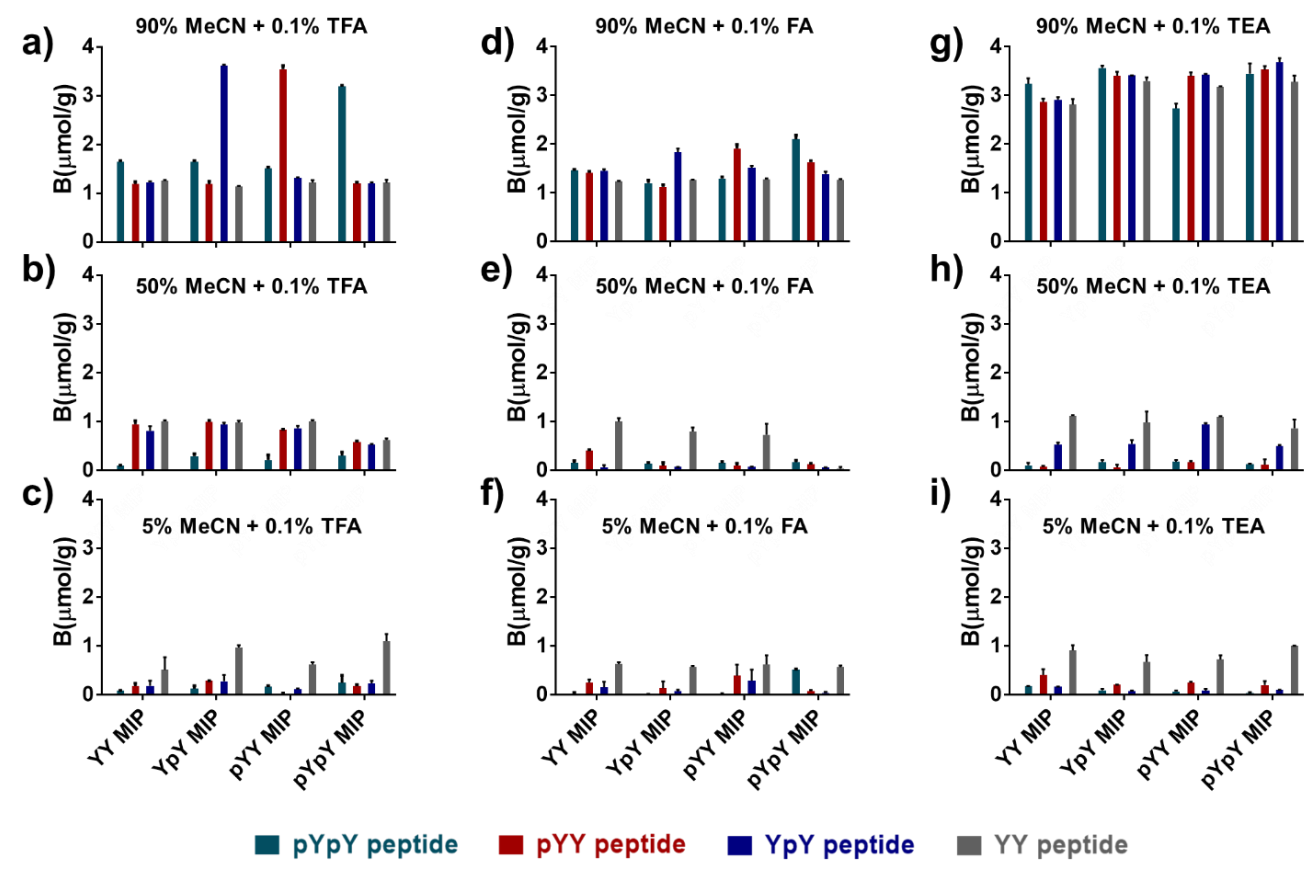

Figure S2: The binding equilibrium test with an equimolar mixture of peptides, GADDSpYpYTAR, GADDSpYYTAR, GADDSYPYTAR, and GADDSYYTAR using sequence specific MIPs in nine different conditions: a. $90 \% \mathrm{MeCN}+0.1 \%$ TFA, b. $50 \% \mathrm{MeCN}+0.1 \%$ TFA, c. $5 \% \mathrm{MeCN}+0.1 \%$ TFA, d. $90 \% \mathrm{MeCN}+0.1 \%$ FA, e. $50 \% \mathrm{MeCN}+0.1 \%$ FA, f. $5 \% \mathrm{MeCN}+0.1 \%$ $\mathrm{FA}$, g. $90 \% \mathrm{MeCN}+0.1 \% \mathrm{TEA}$, h. $50 \% \mathrm{MeCN}+0.1 \% \mathrm{TEA}$, i. $5 \% \mathrm{MeCN}+0.1 \% \mathrm{TEA}$. All experiments were recorded as three replicas. 

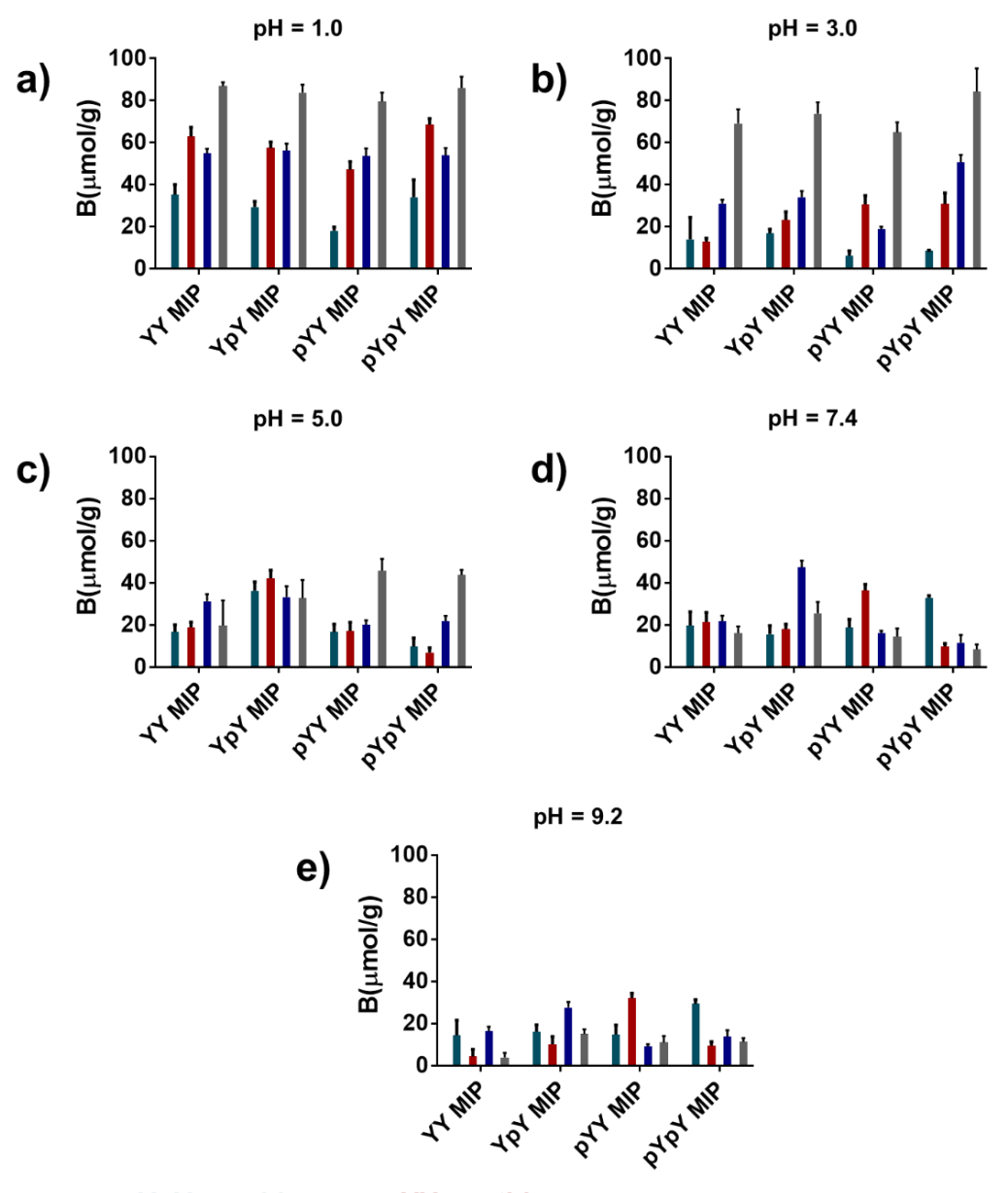

pYpY peptide

pYY peptide

YpY peptide

YY peptide

Figure S3: The binding equilibrium test with an equimolar mixture of peptides, GADDSpYpYTAR, GADDSpYYTAR, GADDSYPYTAR, and GADDSYYTAR using sequence specific MIPs in aqueous buffers: $a . p H=1.0$, b. $p H=3.0, c . p H=5.0$, d. $\mathrm{pH}=7.4$, e. $\mathrm{pH}=9.2$. All experiments were recorded as three replicas. 
Table S1a: Binding properties of YpY and pYY MIPs in the presence of YpY and pYY peptides

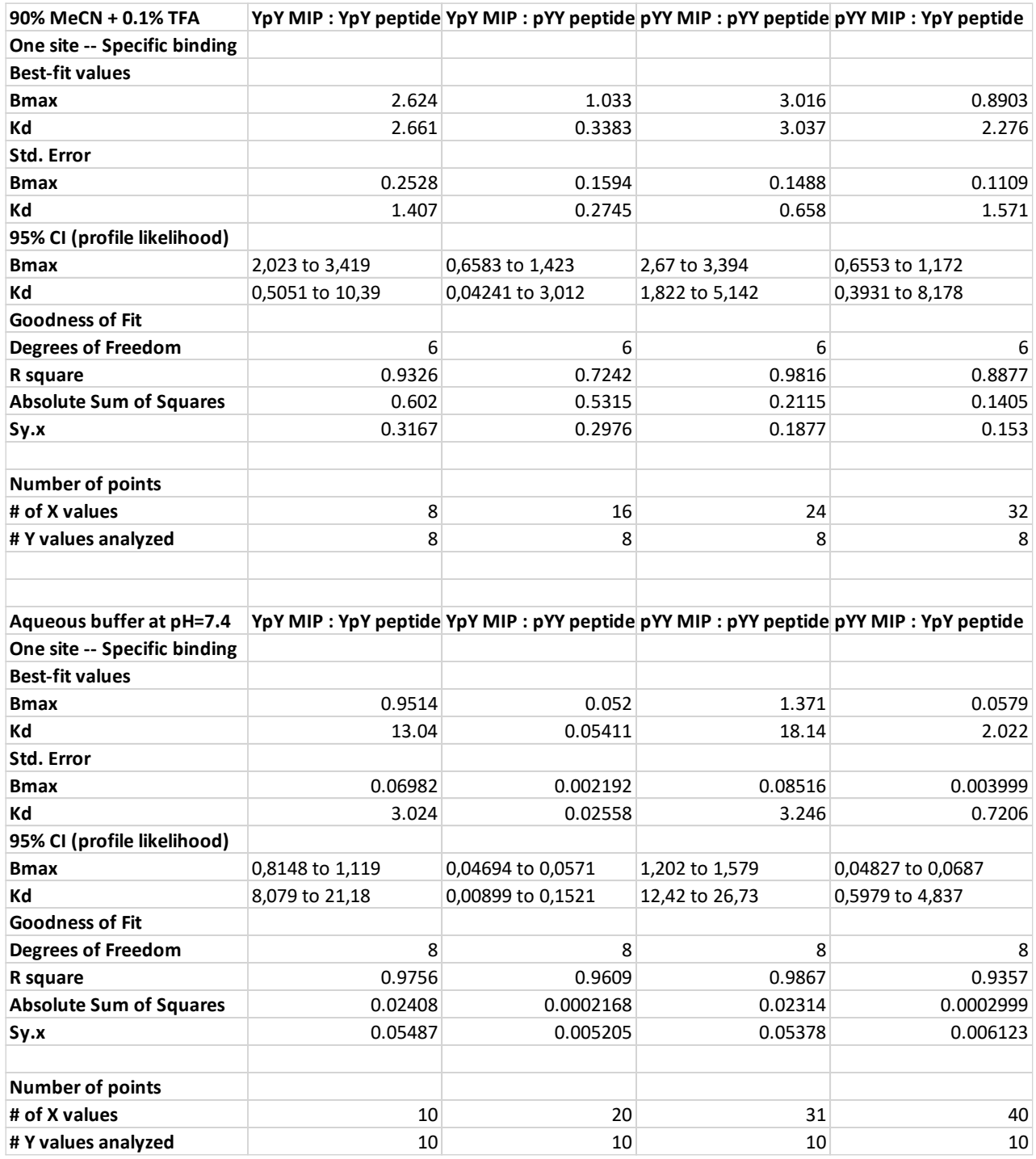


Table S1b: Binding properties of pYpY MIP in the presence of YpY, pYY and pYpY peptides

\begin{tabular}{|c|c|c|c|}
\hline \multirow{2}{*}{$\begin{array}{l}90 \% \text { MeCN }+0.1 \% \text { TFA } \\
\text { One site -- Specific binding }\end{array}$} & \multicolumn{3}{|c|}{ pYpY MIP : pYpY peptide pYpY MIP : pYY peptide pYpY MIP : YpY peptide } \\
\hline & & & \\
\hline \multicolumn{4}{|l|}{ Best-fit values } \\
\hline Bmax & 2.724 & 0.4982 & 0.2264 \\
\hline Kd & 6.587 & 4.833 & 5.3 \\
\hline \multicolumn{4}{|l|}{ Std. Error } \\
\hline Bmax & 0.1501 & 0.07183 & 0.05489 \\
\hline Kd & 1.344 & 2.76 & 4.939 \\
\hline \multicolumn{4}{|l|}{ 95\% Cl (profile likelihood) } \\
\hline Bmax & 2,399 to 3,097 & 0,3513 to 0,6899 & 0,116 to 1,58 \\
\hline Kd & 4,146 to 10,45 & 1,371 to 16,43 & 0,2878 to 408,3 \\
\hline \multicolumn{4}{|l|}{ Goodness of Fit } \\
\hline Degrees of Freedom & 7 & 6 & 6 \\
\hline R square & 0.9817 & 0.8503 & 0.6613 \\
\hline Absolute Sum of Squares & 0.1558 & 0.04777 & 0.026 \\
\hline Sy.x & 0.1492 & 0.08923 & 0.06582 \\
\hline \multicolumn{4}{|l|}{ Number of points } \\
\hline$\#$ of $X$ values & 9 & 17 & 25 \\
\hline \# Y values analyzed & 9 & 8 & 8 \\
\hline & & & \\
\hline & & & \\
\hline
\end{tabular}

Aqueous buffer at pH=7.4 pYpY MIP : pYpY peptide pYpY MIP : pYY peptide pYpY MIP : YpY peptide One site -- Specific binding

Best-fit values

\begin{tabular}{|c|c|c|c|}
\hline \\
\hline Bmax & 0.7622 & 0.08547 & 0.04683 \\
\hline Kd & 13.21 & 0.9831 & 0.2343 \\
\hline \multicolumn{4}{|l|}{ Std. Error } \\
\hline Bmax & 0.0582 & 0.009967 & 0.01025 \\
\hline Kd & 3.174 & 0.9209 & 1.233 \\
\hline \multicolumn{4}{|l|}{ 95\% Cl (profile likelihood) } \\
\hline Bmax & 0,6483 to 0,9037 & 0,06396 to 0,1105 & 0,02506 to 0,07889 \\
\hline Kd & 8,027 to 21,92 & $-0,4463$ to 4,59 & $-1,358$ to 13,59 \\
\hline \multicolumn{4}{|l|}{ Goodness of Fit } \\
\hline Degrees of Freedom & 8 & 8 & 8 \\
\hline R square & 0.9732 & 0.7776 & 0.1664 \\
\hline Absolute Sum of Squares & 0.01683 & 0.002104 & 0.002615 \\
\hline Sy.x & 0.04586 & 0.01622 & 0.01808 \\
\hline \multicolumn{4}{|l|}{ Number of points } \\
\hline$\#$ of $X$ values & 10 & 20 & 30 \\
\hline \# Y values analyzed & 10 & 10 & 10 \\
\hline
\end{tabular}


Enrichment of ZAP-70 decapeptides from complex mixture
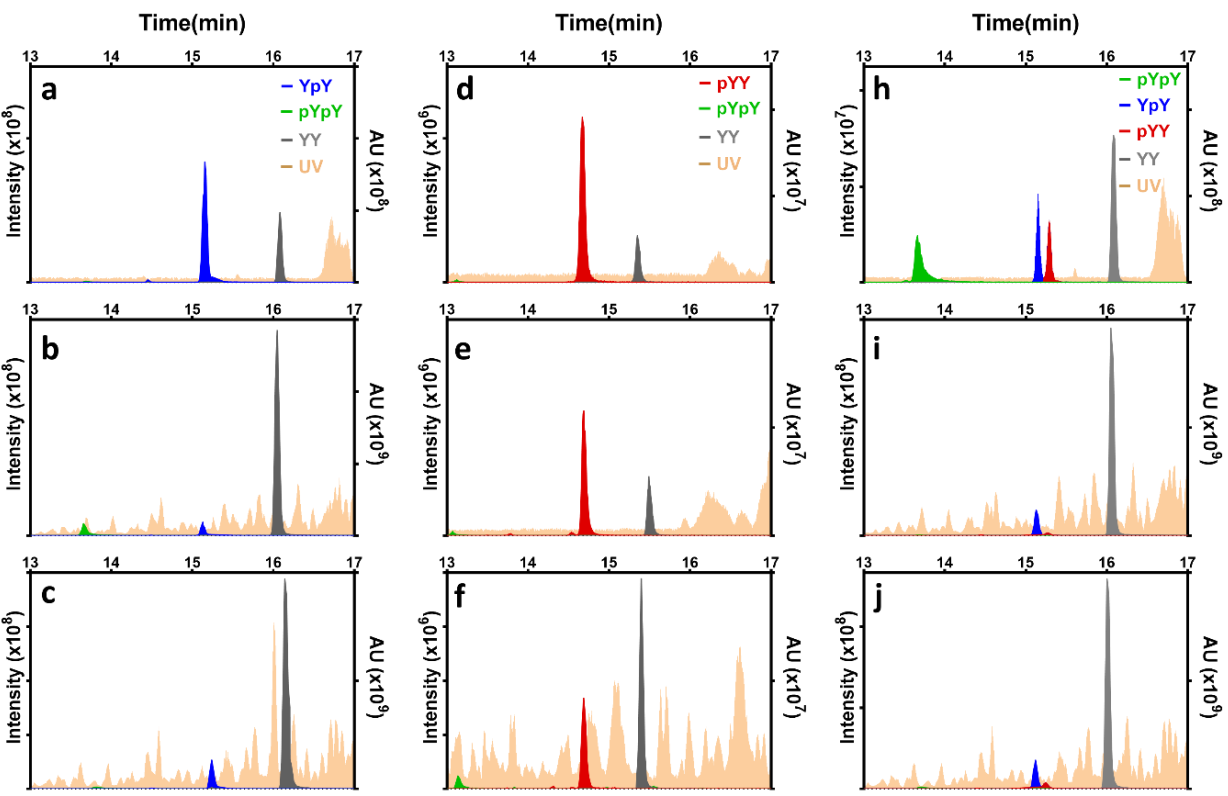

Figure S4: Extracted LC-MS chromatogram of highlighting spiked ZAP-70 peptides (indicated in colors) at 1:100 spiking level corresponding to fractions after enrichment. a YpY-MIP E, b YpY-MIP FT+W, c YpY-MIP R, d pYY-MIP E, e pYY-MIP FT+W, f pYY-MIP R, h pYpY-MIP E, i pYpY-MIP FT+W, j pYpY-MIP R. E = Elution, FT + W = flow-through + loading, $R=$ reference $p Y p Y=$ GADDSpYpYTAR (green), $Y p Y=$ GADDSYpYTAR (blue), $p Y Y=$ GADDSpYYTAR (red), and YY = GADDSYYTAR (gray). The light pink chromatogram in the background stands for full chromatogram. The left $y$-axis shows intensity of spiked peptides, the right $y$-axis refers for the intensity of full chromatogram. 
Enrichment of ZAP-70 phosphopeptides obtained by stimulated Jurkat T-cells.

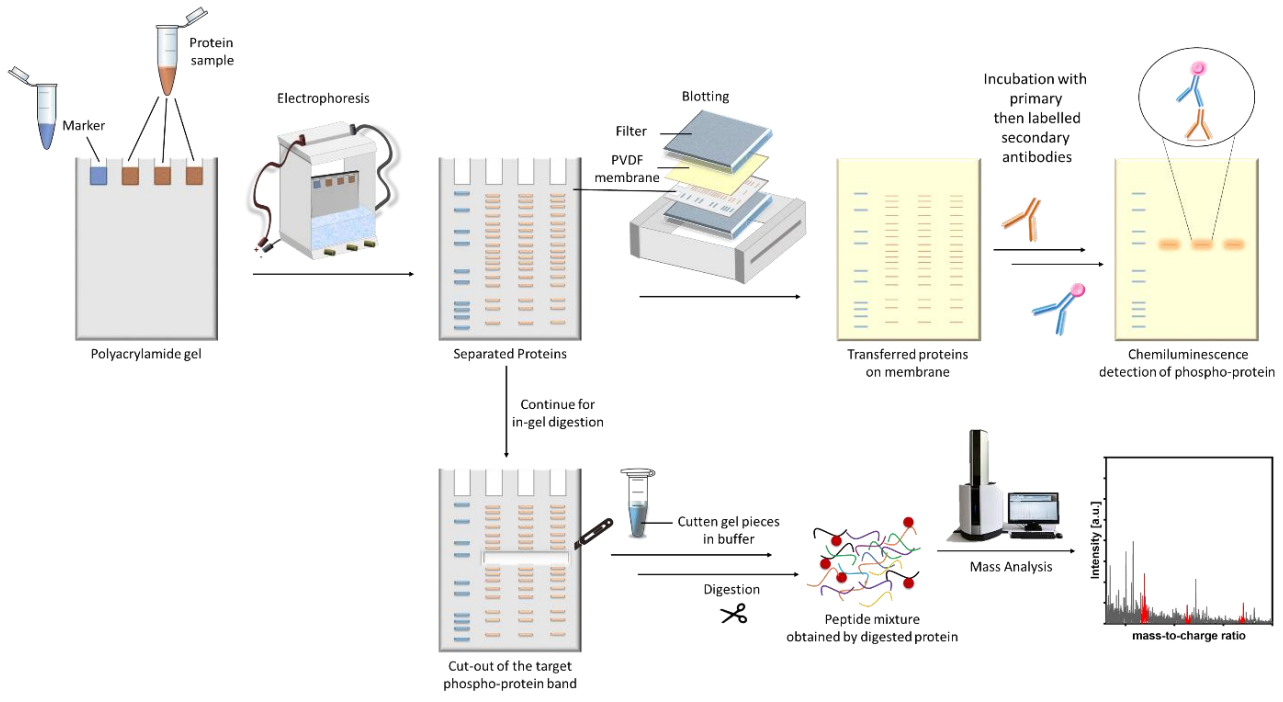

Scheme S2: The schematic explanation of workflow of extraction ZAP-70 phosphopeptides obtained by stimulated Jurkat T-cells: WesternBlot, Immunoprecipitation, In-gel Proteolytic Digestion and Screening

The full length of ZAP-70 protein (residues 1 - 619, Uniprot-P43403)

\begin{tabular}{|c|c|c|c|c|c|c|}
\hline \multirow[t]{2}{*}{1} & MPDPAAHLPF & FYGSISRAEA & EEHLKLAGMA & DGLFLLRQCL & RSLGGYVLSL & VHDVRFHHFP \\
\hline & IERQLNGTYA & IAGGKAHCGP & & & & \\
\hline \multirow[t]{2}{*}{81} & AELCEFYSRD & PDGLPCNLRK & PCNRPSGLEP & QPGVFDCLRD & AMVRDYVRQT & WKLEGEALEO \\
\hline & AlISQAPQVE & KLIATTAHER & & & & \\
\hline \multirow[t]{2}{*}{161} & MPWYHSSLTR & EEAERKLYSG & AQTDGKFLLR & PRKEQGTYAL & SLIYGKTVYH & YLISQDKAGK \\
\hline & YCIPEGTKFD & TLWQLVEYLK & & & & \\
\hline \multirow[t]{2}{*}{241} & LKADGLIYCL & KEACPNSSAS & NASGAAAPTL & PAHPSTLTHP & QRRIDTLNSD & GYTPEPARIT \\
\hline & SPDKPRPMPM & DTSVYESPYS & & & & \\
\hline \multirow[t]{2}{*}{321} & DPEELKDKKL & FLKRDNLLIA & DIELGCGNFG & SVRQGVYRMR & KKQIDVAIKV & LKQGTEKADT \\
\hline & EEMMREAQIM & HQLDNPYIVR & & & & \\
\hline \multirow[t]{2}{*}{401} & LIGVCQAEAL & MLVMEMAGGG & PLHKFLVGKR & EEIPVSNVAE & LLHQVSMGMK & YLEEKNFVHR \\
\hline & DLAARNVLLV & NRHYA*KISDF & & & & \\
\hline \multirow[t]{2}{*}{481} & GLSKALGADD & SYYTARSAGK & WPLKWYAPEC & INFRKFSSRS & DVWSYGVTMW & EALSYGQKPY \\
\hline & KKMKGPEVMA & FIEQGKRMEC & & & & \\
\hline 561 & PPECPPELYA & LMSDCWIYKW & EDRPDFLTVE & QRMRACYYSL & ASKVEGPPGS & TQKAEAACA \\
\hline
\end{tabular}

* Labeled sequence is from 476 to 500 where Y492 and Y493 place. 
Table S2: Tryptic MS-digest list of ZAP-70 protein (obtained by https://prospector.ucsf.edu/prospector/cgibin/msform.cgi?form=msdigest)

\begin{tabular}{|c|c|c|c|c|c|}
\hline $\mathrm{m} / \mathrm{z}$ & $\mathrm{m} / \mathrm{z}$ & Start & End & Missed & Sequence \\
\hline (mi) & (av) & & & Cleavages & \\
\hline 801.5094 & 802.0163 & 187 & 192 & 0 & (K)FLLRPR(K) \\
\hline 827.5098 & 828.0081 & 466 & 472 & 0 & (R)NVLLVNR(H) \\
\hline 866.4618 & 866.9954 & 477 & 484 & 0 & (K)ISDFGLSK(A) \\
\hline 886.5145 & 887.076 & 497 & 504 & 1 & (R)SAGKWPLK(W) \\
\hline 892.4458 & 893.0616 & 354 & 360 & 1 & (R)QGVYRMR(K) \\
\hline 902.5306 & 903.0727 & 370 & 377 & 1 & (K)VLKQGTEK(A) \\
\hline 908.4407 & 909.0609 & 354 & 360 & 1 & (R)QGVYRMR(K) \\
\hline 909.4723 & 910.0922 & 354 & 360 & 1 & (R)QGVYRMR(K) \\
\hline 910.4339 & 911.0709 & 221 & 228 & 0 & (K)YCIPEGTK(F) \\
\hline 914.5669 & 915.1275 & 362 & 369 & 1 & (K)KQIDVAIK(V) \\
\hline 925.4672 & 926.0916 & 354 & 360 & 1 & (R)QGVYRMR(K) \\
\hline 926.4578 & 927.0076 & 18 & 25 & 0 & (R)AEAEEHLK(L) \\
\hline 929.6043 & 930.1914 & 187 & 193 & 1 & (K)FLLRPRK(E) \\
\hline 982.3968 & 983.1136 & 378 & 385 & 0 & (K)ADTEEMMR(E) \\
\hline 995.523 & 996.2209 & 243 & 251 & 0 & (K)ADGLIYCLK(E) \\
\hline 998.3918 & 999.1129 & 378 & 385 & 0 & (K)ADTEEMMR(E) \\
\hline 999.5106 & 1000.1029 & 604 & 613 & 0 & (K)VEGPPGSTQK(A) \\
\hline 1005.471 & 1006.1725 & 595 & 603 & 0 & (R)ACYYSLASK(V) \\
\hline 1011.5582 & 1012.1606 & 152 & 160 & 0 & (K)LIATTAHER(M) \\
\hline 1014.3867 & 1015.1123 & 378 & 385 & 0 & (K)ADTEEMMR(E) \\
\hline 1039.5055 & 1040.1245 & 177 & 186 & 0 & (K)LYSGAQTDGK(F) \\
\hline 1082.553 & 1083.2453 & 56 & 63 & 0 & (R)FHHFPIER(Q) \\
\hline 1095.5582 & 1096.2384 & 125 & 132 & 1 & (R)DYVRQTWK(L) \\
\hline 1099.5201 & 1100.246 & 90 & 99 & 0 & (R)DPDGLPCNLR(K) \\
\hline 1109.6929 & 1110.3907 & 363 & 372 & 1 & (K)QIDVAIKVLK(Q) \\
\hline 1124.5517 & 1125.2995 & 120 & 128 & 1 & (R)DAMVRDYVR(Q) \\
\hline 1126.7194 & 1127.4213 & 363 & 372 & 1 & (K)QIDVAIKVLK(Q) \\
\hline 1140.5466 & 1141.2988 & 120 & 128 & 1 & (R)DAMVRDYVR(Q) \\
\hline 1166.5874 & 1167.3777 & 218 & 228 & 1 & (K)AGKYCIPEGTK(F) \\
\hline 1167.6004 & 1168.2997 & 176 & 186 & 1 & (R)KLYSGAQTDGK(F) \\
\hline 1175.6055 & 1176.3225 & 64 & 75 & 0 & (R)QLNGTYAIAGGK(A) \\
\hline 1192.6321 & 1193.3532 & 64 & 75 & 0 & (R)QLNGTYAIAGGK(A) \\
\hline 1198.644 & 1199.366 & 456 & 465 & 1 & (K)NFVHRDLAAR(N) \\
\hline 1236.7021 & 1237.5566 & 241 & 251 & 1 & (K)LKADGLIYCLK(E) \\
\hline 1276.7082 & 1277.5812 & 26 & 37 & 0 & (K)LAGMADGLFLLR(Q) \\
\hline 1277.6096 & 1278.4842 & 161 & 170 & 0 & (R)MPWYHSSLTR(E) \\
\hline 1292.6126 & 1293.5589 & 593 & 603 & 1 & (R)MRACYYSLASK(V) \\
\hline 1292.7031 & 1293.5805 & 26 & 37 & 0 & (K)LAGMADGLFLLR(Q) \\
\hline 1293.6045 & 1294.4836 & 161 & 170 & 0 & (R)MPWYHSSLTR(E) \\
\hline 1298.5987 & 1299.5028 & 505 & 514 & 0 & (K)WYAPECINFR(K) \\
\hline 1302.5961 & 1303.3791 & 485 & 496 & 0 & (K)ALGADDSYYTAR(S) \\
\hline
\end{tabular}




\begin{tabular}{|c|c|c|c|c|c|}
\hline 1305.6507 & 1306.5327 & 545 & 556 & 0 & (K)GPEVMAFIEQGK(R) \\
\hline 1308.6075 & 1309.5583 & 593 & 603 & 1 & (R)MRACYYSLASK(V) \\
\hline 1321.6457 & 1322.532 & 545 & 556 & 0 & (K)GPEVMAFIEQGK(R) \\
\hline 1326.7641 & 1327.5818 & 466 & 476 & 1 & (R)NVLLVNRHYAK(I) \\
\hline 1334.6852 & 1335.5144 & 451 & 460 & 1 & (K)YLEEKNFVHR(D) \\
\hline 1353.7961 & 1354.605 & 461 & 472 & 1 & (R)DLAARNVLLVNR(H) \\
\hline 1365.7161 & 1366.5692 & 473 & 484 & 1 & (R)HYAKISDFGLSK(A) \\
\hline 1366.7001 & 1367.5538 & 207 & 217 & 0 & (K)TVYHYLISQDK(A) \\
\hline 1426.6936 & 1427.678 & 505 & 515 & 1 & (K)WYAPECINFRK(F) \\
\hline 1442.7526 & 1443.6497 & 194 & 206 & 0 & (K)EQGTYALSLIYGK(T) \\
\hline 1461.7519 & 1462.7213 & 545 & 557 & 1 & (K)GPEVMAFIEQGKR(M) \\
\hline 1477.7468 & 1478.7207 & 545 & 557 & 1 & (K)GPEVMAFIEQGKR(M) \\
\hline 1508.6356 & 1509.6638 & 373 & 385 & 1 & (K)QGTEKADTEEMMR(E) \\
\hline 1514.8326 & 1515.7633 & 42 & 55 & 0 & (R)SLGGYVLSLVHDVR(F) \\
\hline 1515.7108 & 1516.68 & 604 & 619 & 1 & (K)VEGPPGSTQKAEAACA(-) \\
\hline 1524.6305 & 1525.6632 & 373 & 385 & 1 & (K)QGTEKADTEEMMR(E) \\
\hline 1525.6621 & 1526.6945 & 373 & 385 & 1 & (K)QGTEKADTEEMMR(E) \\
\hline 1540.6254 & 1541.6626 & 373 & 385 & 1 & (K)QGTEKADTEEMMR(E) \\
\hline 1541.657 & 1542.6938 & 373 & 385 & 1 & (K)QGTEKADTEEMMR(E) \\
\hline 1554.8203 & 1555.8252 & 229 & 240 & 0 & (K)FDTLWQLVEYLK(L) \\
\hline 1557.6519 & 1558.6932 & 373 & 385 & 1 & (K)QGTEKADTEEMMR(E) \\
\hline 1564.7862 & 1565.9056 & 543 & 556 & 1 & (K)MKGPEVMAFIEQGK(R) \\
\hline 1570.8475 & 1571.8249 & 193 & 206 & 1 & (R)KEQGTYALSLIYGK(T) \\
\hline 1580.7811 & 1581.905 & 543 & 556 & 1 & (K)MKGPEVMAFIEQGK(R) \\
\hline 1582.6777 & 1583.7957 & 76 & 89 & 0 & (K)AHCGPAELCEFYSR(D) \\
\hline 1596.776 & 1597.9044 & 543 & 556 & 1 & (K)MKGPEVMAFIEQGK(R) \\
\hline 1622.8537 & 1623.8605 & 207 & 220 & 1 & (K)TVYHYLISQDKAGK(Y) \\
\hline 1645.7816 & 1646.7645 & 485 & 500 & 1 & (K)ALGADDSYYTARSAGK(W) \\
\hline 1648.7813 & 1649.7651 & 284 & 298 & 0 & (R)IDTLNSDGYTPEPAR(I) \\
\hline 1690.8184 & 1691.8519 & 580 & 592 & 0 & (K)WEDRPDFLTVEQR(M) \\
\hline 1774.8911 & 1776.017 & 1 & 17 & 0 & (-)MPDPAAHLPFFYGSISR(A) \\
\hline 1776.9611 & 1778.2055 & 26 & 41 & 1 & (K)LAGMADGLFLLRQCLR(S) \\
\hline 1792.9561 & 1794.2049 & 26 & 41 & 1 & (K)LAGMADGLFLLRQCLR(S) \\
\hline 1795.9993 & 1797.1609 & 229 & 242 & 1 & (K)FDTLWQLVEYLKLK(A) \\
\hline 1804.8824 & 1805.9537 & 283 & 298 & 1 & (R)RIDTLNSDGYTPEPAR(I) \\
\hline 1816.9017 & 1818.0545 & 1 & 17 & 0 & (-)MPDPAAHLPFFYGSISR(A) \\
\hline 1821.997 & 1823.118 & 177 & 192 & 1 & (K)LYSGAQTDGKFLLRPR(K) \\
\hline 1822.9098 & 1824.1707 & 501 & 514 & 1 & (K)WPLKWYAPECINFR(K) \\
\hline 1826.9218 & 1828.1129 & 386 & 400 & 0 & (R)EAQIMHQLDNPYIVR(L) \\
\hline 1842.9167 & 1844.1123 & 386 & 400 & 0 & (R)EAQIMHQLDNPYIVR(L) \\
\hline 1891.8756 & 1893.1007 & 161 & 175 & 1 & (R)MPWYHSSLTREEAER(K) \\
\hline 1905.9316 & 1907.2148 & 1 & 17 & 0 & (-)MPDPAAHLPFFYGSISR(A) \\
\hline 1907.8705 & 1909.1001 & 161 & 175 & 1 & (R)MPWYHSSLTREEAER(K) \\
\hline 1921.9265 & 1923.2142 & 1 & 17 & 0 & (-)MPDPAAHLPFFYGSISR(A) \\
\hline 1947.9422 & 1949.2523 & 1 & 17 & 0 & (-)MPDPAAHLPFFYGSISR(A) \\
\hline 1963.9371 & 1965.2517 & 1 & 17 & 0 & (-)MPDPAAHLPFFYGSISR(A) \\
\hline
\end{tabular}




\begin{tabular}{|c|c|c|c|c|c|}
\hline 1977.96 & 1979.2384 & 580 & 594 & 1 & (K)WEDRPDFLTVEQRMR(A) \\
\hline 1985.9637 & 1987.2526 & 595 & 613 & 1 & (R)ACYYSLASKVEGPPGSTQK(A) \\
\hline 1993.9549 & 1995.2378 & 580 & 594 & 1 & (K)WEDRPDFLTVEQRMR(A) \\
\hline 1998.0589 & 1999.3569 & 38 & 55 & 1 & (R)QCLRSLGGYVLSLVHDVR(F) \\
\hline 2006.0012 & 2007.2868 & 335 & 353 & 0 & (R)DNLLIADIELGCGNFGSVR(Q) \\
\hline 2015.0855 & 2016.3876 & 38 & 55 & 1 & (R)QCLRSLGGYVLSLVHDVR(F) \\
\hline 2053.0812 & 2054.319 & 133 & 151 & 0 & (K)LEGEALEQAIISQAPQVEK(L) \\
\hline 2150.04 & 2151.3518 & 477 & 496 & 1 & (K)ISDFGLSKALGADDSYYTAR(S) \\
\hline 2162.1023 & 2163.4755 & 334 & 353 & 1 & (K)RDNLLIADIELGCGNFGSVR(Q) \\
\hline 2184.1482 & 2185.566 & 18 & 37 & 1 & (R)AEAEEHLKLAGMADGLFLLR(Q) \\
\hline 2200.1431 & 2201.5654 & 18 & 37 & 1 & (R)AEAEEHLKLAGMADGLFLLR(Q) \\
\hline 2211.1148 & 2212.6082 & 431 & 450 & 0 & (R)EEIPVSNVAELLHQVSMGMK(Y) \\
\hline 2213.0954 & 2214.5891 & 100 & 119 & 0 & $\begin{array}{l}\text { (R)KPCNRPSGLEPQPGVFDCLR(D) } \\
\text { (R) }\end{array}$ \\
\hline 2227.1097 & 2228.6076 & 431 & 450 & 0 & (R)EEIPVSNVAELLHQVSMGMK(Y) \\
\hline 2243.1046 & 2244.607 & 431 & 450 & 0 & (R)EEIPVSNVAELLHQVSMGMK(Y) \\
\hline 2256.1672 & 2257.5757 & 56 & 75 & 1 & (R)FHHFPIERQLNGTYAIAGGK(A) \\
\hline 2270.1499 & 2271.6221 & 152 & 170 & 1 & (K)LIATTAHERMPWYHSSLTR(E) \\
\hline 2286.1448 & 2287.6214 & 152 & 170 & 1 & (K)LIATTAHERMPWYHSSLTR(E) \\
\hline 2367.2159 & 2368.7968 & 430 & 450 & 1 & (K)REEIPVSNVAELLHQVSMGMK(Y) \\
\hline 2383.2108 & 2384.7962 & 430 & 450 & 1 & (K)REEIPVSNVAELLHQVSMGMK(Y) \\
\hline 2399.2057 & 2400.7956 & 430 & 450 & 1 & (K)REEIPVSNVAELLHQVSMGMK(Y) \\
\hline 2446.2363 & 2447.8734 & 221 & 240 & 1 & (K)YCIPEGTKFDTLWQLVEYLK(L) \\
\hline 2468.2532 & 2470.0745 & 401 & 424 & 0 & (R)LIGVCQAEALMLVMEMAGGGPLHK(F) \\
\hline 2484.2481 & 2486.0739 & 401 & 424 & 0 & (R)LIGVCQAEALMLVMEMAGGGPLHK(F) \\
\hline 2500.2431 & 2502.0733 & 401 & 424 & 0 & $\begin{array}{l}\text { (R)LIGVCQAEALMLVMEMAGGGPLHK(F) } \\
\end{array}$ \\
\hline 2516.238 & 2518.0726 & 401 & 424 & 0 & $\begin{array}{l}\text { (R)LIGVCQAEALMLVMEMAGGGPLHK(F) } \\
\end{array}$ \\
\hline 2578.3677 & 2579.9858 & 42 & 63 & 1 & (R)SLGGYVLSLVHDVRFHHFPIER(Q) \\
\hline 2579.3352 & 2580.9155 & 129 & 151 & 1 & (R)QTWKLEGEALEQAIISQAPQVEK(L) \\
\hline 2595.2224 & 2596.9393 & 520 & 541 & 0 & (R)SDVWSYGVTMWEALSYGQKPYK(K) \\
\hline 2596.3617 & 2597.9462 & 129 & 151 & 1 & (R)QTWKLEGEALEQAIISQAPQVEK(L) \\
\hline 2609.314 & 2610.9699 & 335 & 358 & 1 & (R)DNLLIADIELGCGNFGSVRQGVYR(M) \\
\hline 2611.2174 & 2612.9386 & 520 & 541 & 0 & (R)SDVWSYGVTMWEALSYGQKPYK(K) \\
\hline 2618.1144 & 2620.1408 & 558 & 579 & 0 & (R)MECPPECPPELYALMSDCWIYK(W) \\
\hline 2634.1093 & 2636.1402 & 558 & 579 & 0 & (R)MECPPECPPELYALMSDCWIYK(W) \\
\hline 2650.1042 & 2652.1395 & 558 & 579 & 0 & (R)MECPPECPPELYALMSDCWIYK(W) \\
\hline 2663.1799 & 2665.0189 & 76 & 99 & 1 & $\begin{array}{l}\text { (K)AHCGPAELCEFYSRDPDGLPCNLR(K) } \\
\text { (K) }\end{array}$ \\
\hline 2682.3311 & 2684.0019 & 1 & 25 & 1 & (-)MPDPAAHLPFFYGSISRAEAEEHLK(L) \\
\hline 2723.3174 & 2725.1144 & 520 & 542 & 1 & (R)SDVWSYGVTMWEALSYGQKPYKK(M) \\
\hline 2724.3416 & 2726.0394 & 1 & 25 & 1 & (-)MPDPAAHLPFFYGSISRAEAEEHLK(L) \\
\hline 2739.2654 & 2741.0954 & 64 & 89 & 1 & (R)QLNGTYAIAGGKAHCGPAELCEFYSR(D) \\
\hline 2739.3123 & 2741.1138 & 520 & 542 & 1 & (R)SDVWSYGVTMWEALSYGQKPYKK(M) \\
\hline 2756.2919 & 2758.1261 & 64 & 89 & 1 & (R)QLNGTYAIAGGKAHCGPAELCEFYSR(D) \\
\hline 2774.2155 & 2776.3294 & 557 & 579 & 1 & (K)RMECPPECPPELYALMSDCWIYK(W) \\
\hline 2785.3695 & 2787.2774 & 100 & 124 & 1 & (R)KPCNRPSGLEPQPGVFDCLRDAMVR(D) \\
\hline 2790.2104 & 2792.3288 & 557 & 579 & 1 & (K)RMECPPECPPELYALMSDCWIYK(W) \\
\hline 2790.3008 & 2792.2038 & 378 & 400 & 1 & $\begin{array}{l}\text { (K)ADTEEMMREAQIMHQLDNPYIVR(L) } \\
\end{array}$ \\
\hline
\end{tabular}




\begin{tabular}{|c|c|c|c|c|c|}
\hline 2790.4349 & 2792.1808 & 194 & 217 & 1 & (K)EQGTYALSLIYGKTVYHYLISQDK(A) \\
\hline 2801.3644 & 2803.2768 & 100 & 124 & 1 & (R)KPCNRPSGLEPQPGVFDCLRDAMVR(D) \\
\hline 2806.2053 & 2808.3282 & 557 & 579 & 1 & (K)RMECPPECPPELYALMSDCWIYK(W) \\
\hline 2806.2957 & 2808.2031 & 378 & 400 & 1 & $\begin{array}{l}\text { (K)ADTEEMMREAQIMHQLDNPYIVR(L) } \\
\end{array}$ \\
\hline 2813.3716 & 2815.1997 & 1 & 25 & 1 & (-)MPDPAAHLPFFYGSISRAEAEEHLK(L) \\
\hline 2822.2906 & 2824.2025 & 378 & 400 & 1 & (K)ADTEEMMREAQIMHQLDNPYIVR(L) \\
\hline 2829.3665 & 2831.199 & 1 & 25 & 1 & (-)MPDPAAHLPFFYGSISRAEAEEHLK(L) \\
\hline 2838.2855 & 2840.2019 & 378 & 400 & 1 & (K)ADTEEMMREAQIMHQLDNPYIVR(L) \\
\hline 2855.3821 & 2857.2372 & 1 & 25 & 1 & (-)MPDPAAHLPFFYGSISRAEAEEHLK(L) \\
\hline 2871.377 & 2873.2366 & 1 & 25 & 1 & (-)MPDPAAHLPFFYGSISRAEAEEHLK(L) \\
\hline 2873.4423 & 2875.3535 & 431 & 455 & 1 & (R)EEIPVSNVAELLHQVSMGMKYLEEK(N) \\
\hline 2889.4373 & 2891.3529 & 431 & 455 & 1 & (R)EEIPVSNVAELLHQVSMGMKYLEEK(N) \\
\hline 2905.4322 & 2907.3522 & 431 & 455 & 1 & 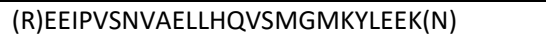 \\
\hline 3012.5905 & 3014.7737 & 401 & 429 & 1 & 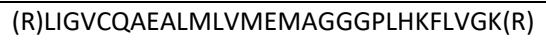 \\
\hline 3028.5855 & 3030.7731 & 401 & 429 & 1 & $\begin{array}{l}\text { (R)LIGVCQAEALMLVMEMAGGGPLHKFLVGK(R) } \\
\end{array}$ \\
\hline 3041.4494 & 3043.3219 & 252 & 282 & 0 & (K)EACPNSSASNASGAAAPTLPAHPSTLTHPQR(R) \\
\hline 3044.5804 & 3046.7724 & 401 & 429 & 1 & (R)LIGVCQAEALMLVMEMAGGGPLHKFLVGK(R) \\
\hline 3045.6215 & 3047.4568 & 133 & 160 & 1 & (K)LEGEALEQAIISQAPQVEKLIATTAHER(M) \\
\hline 3060.5753 & 3062.7718 & 401 & 429 & 1 & $\begin{array}{l}\text { (R)LIGVCQAEALMLVMEMAGGGPLHKFLVGK(R) } \\
\end{array}$ \\
\hline 3072.456 & 3074.4631 & 516 & 541 & 1 & (K)FSSRSDVWSYGVTMWEALSYGQKPYK(K) \\
\hline 3088.4509 & 3090.4624 & 516 & 541 & 1 & (K)FSSRSDVWSYGVTMWEALSYGQKPYK(K) \\
\hline 3197.5505 & 3199.5106 & 252 & 283 & 1 & (K)EACPNSSASNASGAAAPTLPAHPSTLTHPQRR(I) \\
\hline 3209.5017 & 3211.6131 & 299 & 326 & 0 & (R)ITSPDKPRPMPMDTSVYESPYSDPEELK(D) \\
\hline 3225.4966 & 3227.6125 & 299 & 326 & 0 & $\begin{array}{l}\text { (R)ITSPDKPRPMPMDTSVYESPYSDPEELK(D) } \\
\text { (R) }\end{array}$ \\
\hline 3241.4915 & 3243.6119 & 299 & 326 & 0 & $\begin{array}{l}\text { (R)ITSPDKPRPMPMDTSVYESPYSDPEELK(D) } \\
\text { (R) }\end{array}$ \\
\hline 3293.5976 & 3295.8124 & 90 & 119 & 1 & (R)DPDGLPCNLRKPCNRPSGLEPQPGVFDCLR(D) \\
\hline 3452.6236 & 3454.8774 & 299 & 328 & 1 & (R)ITSPDKPRPMPMDTSVYESPYSDPEELKDK(K) \\
\hline 3468.6185 & 3470.8768 & 299 & 328 & 1 & (R)ITSPDKPRPMPMDTSVYESPYSDPEELKDK(K) \\
\hline 3484.6134 & 3486.8762 & 299 & 328 & 1 & $\begin{array}{l}\text { (R)ITSPDKPRPMPMDTSVYESPYSDPEELKDK(K) } \\
\text { (R) }\end{array}$ \\
\hline
\end{tabular}

Table S3: Phosphorylation states in the kinase domain

\begin{tabular}{|c|c|c|}
\hline $\begin{array}{c}\text { Phosphorylation Site on Y492, Y493 } \\
\text { in kinase domain }\end{array}$ & Peptide Sequence & $\begin{array}{c}\text { mass-to- } \\
\text { charge ratio }\end{array}$ \\
\hline $\mathbf{Y}^{\mathbf{0}} \mathbf{Y}^{\mathbf{0}}$ & ALGADDSYYTAR & 1302.59 \\
\hline $\mathbf{Y}^{\mathbf{P}} \mathbf{Y}^{\mathbf{0}}$ & ALGADDSPYYTAR & 1382.35 \\
\hline $\mathbf{Y}^{\mathbf{0}} \mathbf{Y}^{\mathbf{P}}$ & ALGADDSYPYTAR & 1382.35 \\
\hline $\mathbf{Y}^{\mathbf{P}} \mathbf{Y}^{\mathbf{P}}$ & ALGADDSPYPYTAR & 1462.12 \\
\hline
\end{tabular}


CD3 stimulated, digested ZAP70 p-protein

\section{ALGADDSYYTAR}

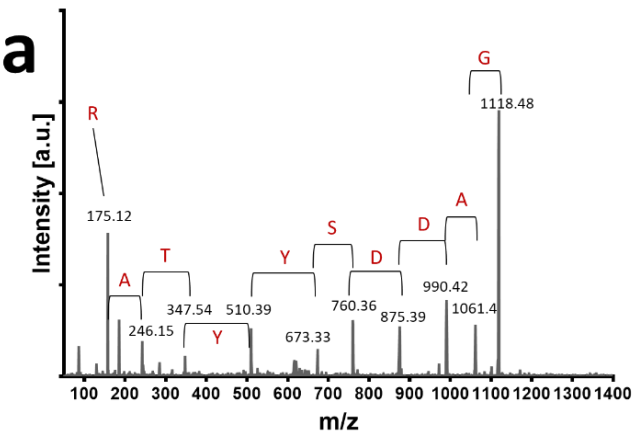

$\mathrm{CD} 3+\mathrm{H}_{2} \mathrm{O}_{2}$ stimulated, digested ZAP70 p-protein

ALGADDSYYTAR

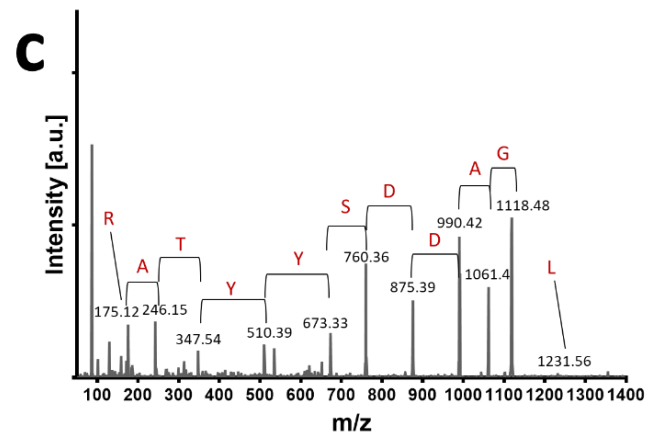

CD3 stimulated, digested ZAP70 p-protein

$$
\text { ALGADDSpYpYTAR }
$$

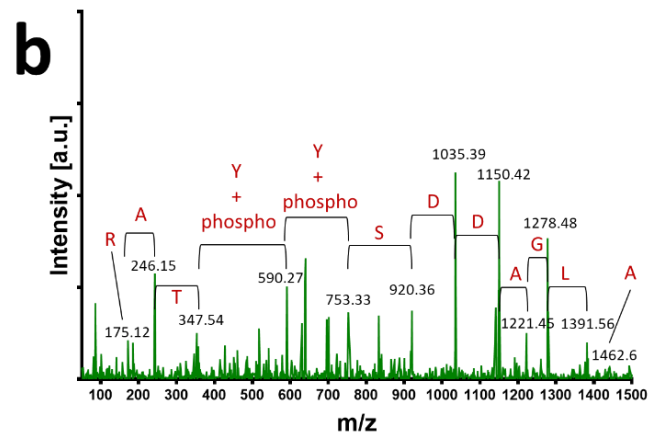

$\mathrm{CD} 3+\mathrm{H}_{2} \mathrm{O}_{2}$ stimulated, digested ZAP70 p-protein

$$
\text { ALGADDSpYpYTAR }
$$

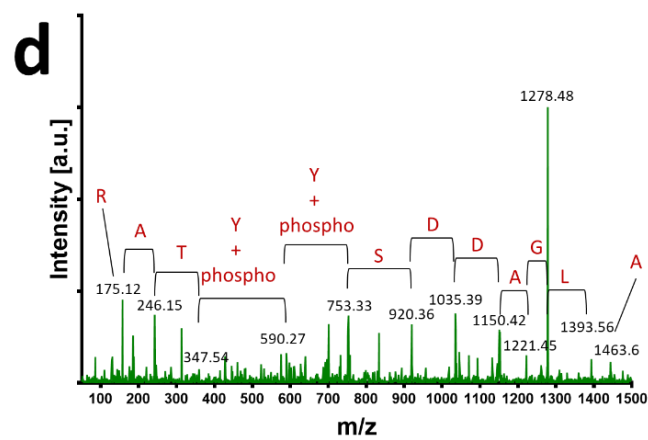

Figure S5: Fragmentation patterns of ZAP-70 phosphopeptides obtained by stimulated Jurkat T-cells. Peptides obtained CD3 stimulation a. ALGADDSYYTAR, b. ALGADDSpYpYTAR, Peptides obtained $\mathrm{CD} 3+\mathrm{H}_{2} \mathrm{O}_{2}$ stimulation c. ALGADDSYYTAR, d. ALGADDSPYPYTAR. 

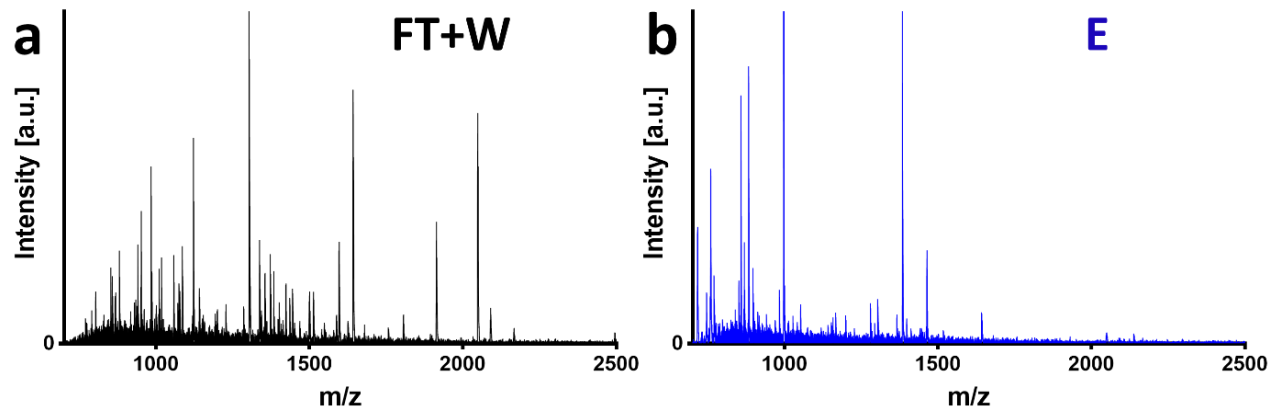

pYY MIP - enrichment fractions

PYY MIP - enrichment fractions
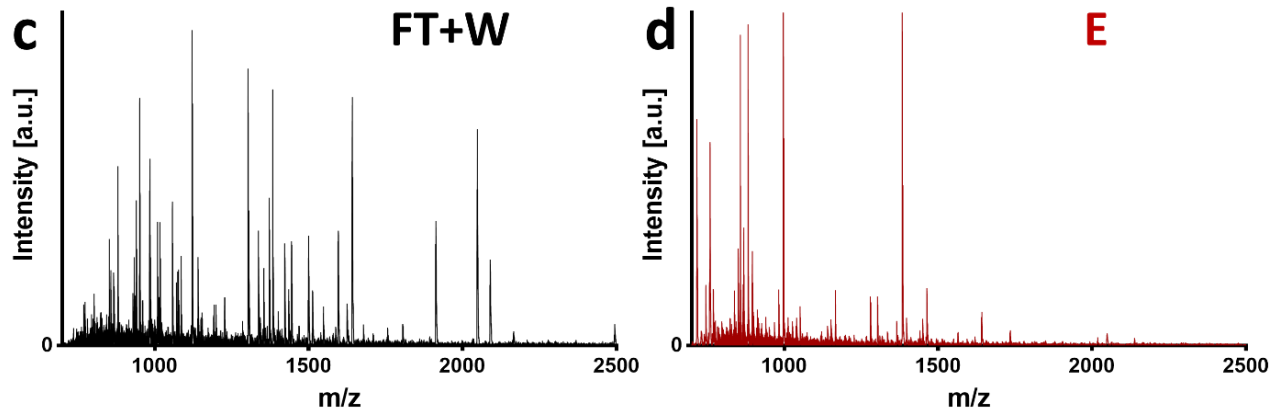

Figure S6: MALDI-MS profiles. a YpY MIP / FT+W fraction b YpY MIP / E fraction c pYY MIP / FT+W fraction d pYY MIP / E fraction. $\mathrm{FT}+\mathrm{W}$ is flow through + washing and $\mathrm{E}$ is for elution. 
IV 



\section{EDGE ARTICLE}

\section{Check for updates}

Cite this: Chem. Sci., 2020, 11, 4246

¿ All publication charges for this article have been paid for by the Royal Society of Chemistry
Received 23rd December 2019

Accepted 1st April 2020

DOI: $10.1039 /$ c9sc06508c

rsc.li/chemical-science

\section{High salt compatible oxyanion receptors by dual ion imprinting $\dagger$}

\author{
Sudhirkumar Shinde, (D) $\ddagger$ Mona Mansour, (D) $\S$ Anil Incel, (D) Liliia Mavliutova, (D) \\ Celina Wierzbicka (iD) and Börje Sellergren (D)*
}

\section{Introduction}

Anion recognition drives a multitude of processes which are crucial for the living cell. ${ }^{1,2}$ The work horses in these processes are anion receptors refined by evolution to discriminatively bind anions in water. The performance of these receptors becomes particularly impressive when considering that they can exert their action in extreme environments in highly competitive media of high ionic strength. An often cited example in this regard refers to the proteins designed to specifically bind the pyramidal oxoanions phosphate, sulfate and arsenate. ${ }^{2-4}$ Discrimination between them occurs predominantly through multiple complementary hydrogen bonding involving main chain amides (nests) in a water poor microenvironment with less contribution by charged residues. ${ }^{5}$ The ability of phosphate binding protein to select phosphate over the isosteric arsenate with a selectivity factor of 4500 and sulfate binding protein to bind sulfate specifically with a binding constant $K_{\mathrm{a}}=10^{6} \mathrm{M}^{-1}$ in water ( $\mathrm{pH}$ 5-8) reflect the perfection of these receptors. Hence, this overturns the Hofmeister series of the salting out tendency for anions which otherwise falls in the order: $\mathrm{SO}_{4}{ }^{2-}>\mathrm{HPO}_{4}{ }^{2-}>$ acetate.

From the above perspective, efforts to design biomimetic anion hosts fall short. Anion recognition by neutral synthetic

Department of Biomedical Sciences, Faculty of Health and Society, Malmö University, 20506 Malmö, Sweden. E-mail: borje.sellergren@mau.se

$\dagger$ Electronic supplementary information (ESI) available: Experimental section and supporting data. See DOI: $10.1039 / \mathrm{c} 9 \mathrm{sc06508 \textrm {c }}$

† Current address: School of Chemistry and Chemical Engineering, Queens University Belfast, Northern Ireland, UK.

$\S$ Current address: Analysis and evaluation department, Egyptian petroleum research institute, 1 Ahmed el zomor street, Nasr city, Cairo, Egypt. receptors can be highly selective but the receptors are challenging to construct since hydrogen bonding is considerably weaker in polar solvents., ${ }^{2,6}$ Moreover, ion recognition is complicated by the necessary presence of their counterions. For example, anion binding is electrostatically screened in high salt media, a situation commonly exploited in ion exchange chromatography for modulating ion retention. ${ }^{7}$ To avoid this screening effect, recognition at the air water interface ${ }^{8}$ or dual ion receptors ${ }^{9,10}$ comprising preorganized receptors for both anion and cation have been reported. The latter employ combinations of known cation or anion recognition motifs held together by appropriately designed spacers. In spite of the promising progress this approach suffers from significant synthetic challenges in correctly placing the anion and cation hosts to match the ion separation distance, hence requiring a priori knowledge about ion pair solvation i.e. contact or solvent separated.

This dilemma we believe can be addressed by turning from bottom up design to top down strategies relying on selfassembly. Hence potent anion receptors can be prepared by polymerizing host monomers and a crosslinker in presence of the anion guest followed by guest removal. ${ }^{11-21}$ The anion preorganizes the host which is covalently fixed in a macromolecular scaffold with tunable local polarity. Permanent imprinted sites are achieved post template removal featuring enhanced affinity for the templated ion. This concept can be further exploited to incorporate sites for the counterion. Hence, combining anion and cation host monomers we anticipate will spontaneously lead to dual ion receptors with optimally adjusted interhost distance for recognition in competitive aqueous media. ${ }^{22}$ To demonstrate this concept, we have here compared urea-based imprinted monoion phosphate receptors 


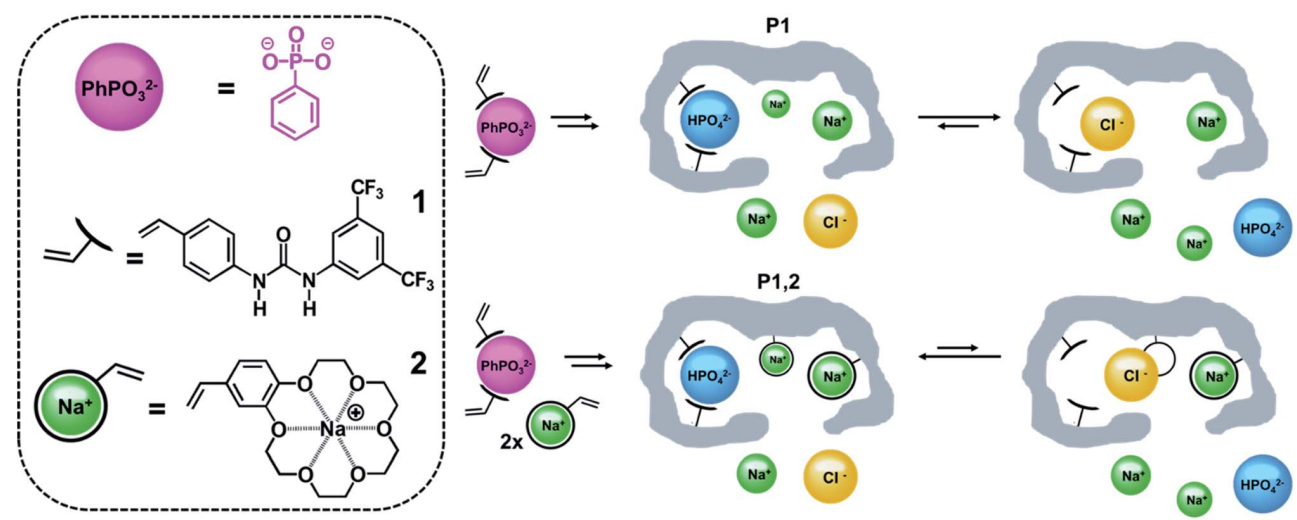

Fig. 1 Template $\left(\mathrm{PPA}=\mathrm{PhPO}_{3}{ }^{2-}\right.$ ) and host monomers (left) and principle of monoanion (top) and dual ion (bottom) imprinting with anticipated phosphate recognition of corresponding imprinted polymers in high salt media.

with corresponding dual ion receptors targeting sodium or potassium salts (Fig. 1).

\section{Results and discussion}

\section{Solution complex formation and polymer preparation}

The dual ion imprinting receptor was constructed using 1,3diarylurea monomer $\mathbf{1}$ as anion host and vinyl-benzo-18-crown6 (monomer 2$)^{23}$ as polymerizable macrocyclic cation host (Fig. 1). The former acts as twofold hydrogen bond donor to complex oxyanion guests and can be used to prepare high affinity anion receptors for highly diverse set of guests e.g. phosphopeptides, sulfopeptides, phospholipids, sugar acids, carboxylates. ${ }^{14-17,19}$ Complexation can be easily induced by proton transfer from the acid to bulky amine bases such as pentamethylpiperidine (PMP) or by the use of quaternary ammonium counterions (e.g. tetrabutylammonium, TBA) which moreover have been shown to produce pronounced counterion memory effects. ${ }^{14,24}$

Crownethers on the other hand are macrocyclic hosts complexing size matched metal ions with a $1: 1$ host guest stoichiometry. ${ }^{25-27}$ The 18 -membered ring macrocycle 18-crown-6 (18C6) features a cavity size matching the abundant alkali cations sodium and potassium but with a strongly solvent dependent affinity (entries 3-5 in Table 1). Exposed to water its amphiphilic nature leads to cavity collapse and loss of binding but this may be counteracted by embedding it in scaffolds providing lower polarity microenvironments.

To investigate whether monomer 2 could promote the association of host monomer $\mathbf{1}$ with the mono-sodium salt of phenylphosphonic acid (PPA-Na) we carried out ${ }^{1} \mathrm{H}-\mathrm{NMR}$ titrations in DMSO- $d_{6}$ (Fig. S1 and S2 $\dagger$ ) and compared it with our previous records for the corresponding titration with PPA TBA (Table 1). The titration of 1 with PPA-TBA in DMSO- $d_{6}$ could previously be modelled using a one site host-guest model resulting in a binding constant $K_{\mathrm{a}}=7005 \mathrm{M}^{-1}$.
A different result was obtained using the crown-ether stabilized guest (Fig. S1 and S2 $\dagger$ ). Steep downfield shifts were observed for the urea protons $H_{\mathrm{a}}$ and $H_{\mathrm{b}}$ which inflected abruptly at a $1: 1$ host guest ratio with only minor changes observed beyond this guest level. This isotherm was best fitted with the Hill equation resulting in a significantly higher association constant of $K_{\mathrm{a}}=11181 \mathrm{M}^{-1}$. A Hill coefficient of 1.7 indicates a strongly positive cooperativity, possibly caused by the multiple equilibria involved in forming the higher order complexes.

Imprinted and nonimprinted polymers were prepared and characterised using the urea host monomers $\mathbf{1}$ and $\mathbf{2}$ as listed in Table $\mathrm{S} 1 \dagger$ (Fig. 1). Nonimprinted polymers $\left(P_{\mathrm{N}}\right)$ were prepared identically to the imprinted polymers but omitting the template. Characterisation of the polymers by scanning electron microscopy (SEM) (Fig. S3 $\dagger$ ), elemental analysis and transmission FTIR (Fig. S4 $\dagger$ ) gave data supporting the formation of polymers with a macroporous morphology, a stoichiometric monomer incorporation reflecting the feed ratio and a successful template removal.

\section{pH Dependence of ion recognition in buffer}

To evaluate the anion recognition properties of the materials we assessed their ability to bind both organic (PPA and

Table 1 Association constants for complexes of template and functional monomers in different solvents

\begin{tabular}{lllll}
\hline Entry & Guest & Host & $K_{\mathrm{a}}\left(\mathrm{M}^{-1}\right)$ & Solvent \\
\hline 1 & PPA-TBA & 1 & $7005 \pm 985^{a, c}$ & DMSO- $d_{6}$ \\
2 & $\mathrm{PPA} \cdot \mathrm{Na}-18 \mathrm{C} 6$ & 1 & $11181 \pm 1655^{a}$ & DMSO- $d_{6}$ \\
3 & $\mathrm{Na}^{+}$ & $18 \mathrm{C} 6$ & $25^{b}$ & DMSO \\
4 & $\mathrm{Na}^{+}$ & $18 \mathrm{C} 6$ & $31622^{b}$ & MeCN \\
5 & $\mathrm{Na}^{+}$ & $18 \mathrm{C} 6$ & $6.3^{b}$ & $\mathrm{H}_{2} \mathrm{O}$
\end{tabular}

${ }^{a}$ Determined by ${ }^{1} \mathrm{H}$-NMR titrations from the average of the individual complexation induced shifts of both urea protons. ${ }^{b}$ Determined by conductometry of sodiumperchlorate solutions. ${ }^{28}{ }^{c}$ See ref. 15. 
phenylsulphonic acid PSA) and inorganic $\left(\mathrm{HPO}_{4}{ }^{2-}\right.$ and $\left.\mathrm{SO}_{4}{ }^{2-}\right)$ oxyanions. The polymers were incubated in buffers adjusted to different $\mathrm{pH}$ values in presence of the sodium salts of the anions. Fig. 2A and S5 $\uparrow$ show the uptake of PPA and PSA by the different polymers in the $\mathrm{pH}$ range 1-9.

First, we note that binding increases with increasing $\mathrm{pH}$, the trend being more pronounced for PPA compared to PSA. This is most likely related to the different protonation states of the two anions and the stronger hydration tendency of sulphate. The steepest increase in anion uptake is observed for the imprinted materials P1 and P1,2 containing anion host monomer 1 whereas the polymers prepared using host monomer 2 alone as in P2 did not display strong imprinting. In agreement with our previous observations, ${ }^{15}$ acidic conditions favored selectivity for sulphonate. This is reflected in the selectivity factor shown in Fig. 2B. Interestingly, only $\mathrm{P} 1,2$ featured a $\mathrm{PhSO}_{3} / \mathrm{PhPO}_{3}$ selectivity factor exceeding 1 under such conditions.

We then investigated the ion selectivity of all polymers in buffer $\mathrm{pH}$ 9. Binding of the inorganic anions was measured by conductometry with both anions carrying a net 2-fold negative charge (Fig. 3A). As expected, the polymers showed a preference for phosphate over sulphate and imprinted polymers showed a significantly larger uptake than the nonimprinted reference polymers. To prove the presence of dual ion receptors we then performed the same experiment in presence of $1 \mathrm{M} \mathrm{NaCl}$, corresponding to $\mathrm{ca}$. $1 / 5$ th of a saturated salt solution (brine). As seen in Fig. 3A the salt had a positive effect on the ion binding to $\mathrm{P} 1,2$ whereas binding to $\mathrm{P} 1$ appeared less affected. These results are contrary to salt induced ionic screening effects seen in charge driven molecular recognition. We attribute this effect to the colocalization of both cation and anion hosts caused by the imprinting process.

\section{Adsorption isotherms and binding parameters in low and high salt media}

The binding-energy distributions of the polymers P1 and P1,2 and their corresponding nonimprinted polymers are given by single-component adsorption isotherms determined by batch equilibration in $0.1 \mathrm{M}$ sodium bicarbonate buffer at $\mathrm{pH} 9$

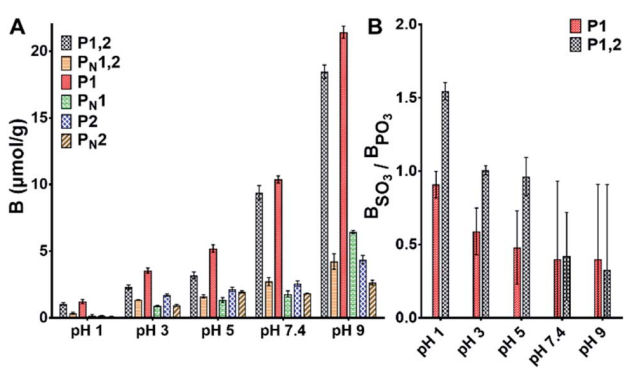

Fig. 2 (A) Binding of PPA $(0.6 \mathrm{mM})$ on PPA imprinted polymers in buffers of different $\mathrm{pH}$. (B) Sulfo-selectivity expressed as the ratio of bound PSA to PPA based on the binding data in Fig. $2 \mathrm{~A}$ and S5. $\dagger$
A
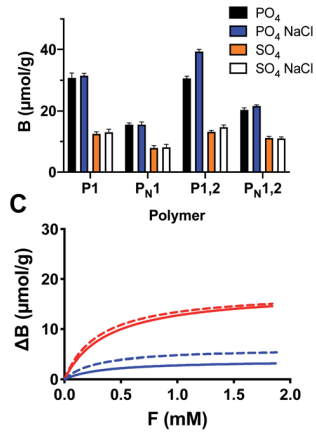

B
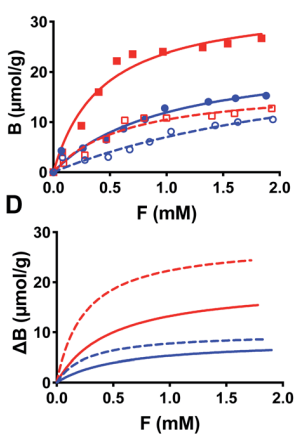

Fig. 3 (A) Binding of phosphate and sulphate $(0.6 \mathrm{mM})$ on PPA imprinted and nonimprinted polymers in $0.1 \mathrm{M}$ sodium bicarbonate buffer at $\mathrm{pH} 9$ in absence and presence of salt (1 $\mathrm{M} \mathrm{NaCl}$ ). (B) Equilibrium binding isotherms of PPA (red curves, squares) and PSA (blue curves, circles) on polymer P1 (solid curves, filled symbols) and PN1 (dashed curves, open symbols) in $0.1 \mathrm{M}$ sodium bicarbonate buffer $\mathrm{pH}$ 9. (C and D) show the binding isotherms on P1 (C) and P1,2 (D) corrected for binding to the nonimprinted polymers $\mathrm{PN} 1$ and $\mathrm{PN} 1,2$ with the dashed curves representing anion binding in presence of $1 \mathrm{M} \mathrm{NaCl}$.

(Fig. 3B and S6†). The imprinted polymers consistently displayed a higher uptake of both PPA and PSA compared to their nonimprinted counterparts with the isotherms showing clear saturation behaviour. This contrasts with the results for the nonimprinted polymers were only the PPA isotherms displayed curvature whereas those of PSA appeared linear (Fig. S6 $\%$ ). As expected from the initial batch binding experiments in Fig. 3A, the saturation capacity for PPA is overall higher than that for PSA. Fitting these data with the one site host-guest model resulted in the curves shown in Fig. $56 \dagger$ with the fitting parameters $K_{\mathrm{a}}$ and $B_{\max }$ given in Table S2. $\dagger$ The preference for PPA is reflected in the higher association constants recorded for this anion again with the highest values obtained for the imprinted polymers.

The same experiment was then performed in presence of $1 \mathrm{M}$ $\mathrm{NaCl}$. Overall, this resulted in little or no change in binding affinity for P1 whereas P1,2 now bound the oxyanions more tightly. This agrees with the results reported in Fig. $3 \mathrm{~A}$ and shows a clear positive effect of salt on the anion binding affinity of P1,2. Hence, $\mathrm{P} 1,2$ showed the highest affinity with a $K_{\mathrm{a}}=3700 \mathrm{M}^{-1}$ and a $B_{\max }=45 \mu \mathrm{mol} \mathrm{g}^{-1}$ for PPA (Table S2 $\dagger$ ).

To highlight the contribution to binding caused by the template effect we subtracted binding to the nonimprinted from the imprinted polymer (assuming the former to reflect the nonspecific binding contribution) (Fig. 4) and compared the resulting binding parameters in graphic format (Fig. 3C and D). The graphs offer a convincing evidence for the synergistic effect of the dual ion host on the binding affinity and capacity. Whereas this host $(\mathrm{P} 1,2)$ displayed a concomitant increase in both binding constant $\left(K_{\mathrm{a}}=2000\right.$ to $4000 \mathrm{M}^{-1}$ for PPA) and saturation capacity $\left(B_{\max }=20\right.$ to $\left.28 \mu \mathrm{mol} \mathrm{g}^{-1}\right)$ in the presence of 
A

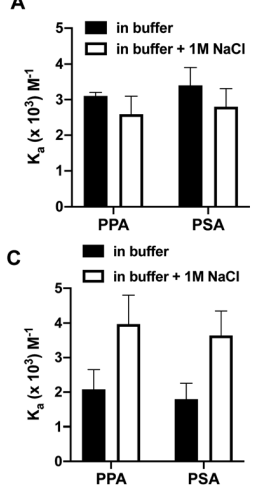

B

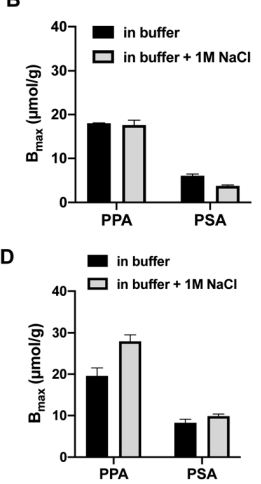

Fig. 4 Association constants $\left(K_{\mathrm{a}}\right)$ (A and $C$ ) and binding capacities $\left(B_{\max }\right)(\mathrm{B}$ and $\mathrm{D})$ for PPA and PSA interacting with $\mathrm{P} 1$ (A and $\mathrm{B}$ ) and $\mathrm{P} 1,2$ $(C$ and $D)$ in $0.1 \mathrm{M}$ sodium bicarbonate buffer $\mathrm{pH} 9$ with or without addition of extra salt $(1 \mathrm{M} \mathrm{NaCl})$. The binding parameters were derived from the corrected curves in Fig. $3 C$ and D.

$1 \mathrm{M} \mathrm{NaCl}$, the corresponding mono-ion host (P1) showed a decreased PPA affinity $\left(K_{\mathrm{a}}=3100\right.$ to $\left.2600 \mathrm{M}^{-1}\right)$ and no change in capacity $\left(B_{\max }=18 \mu \mathrm{mol} \mathrm{g}^{-1}\right)$.

\section{Conclusions}

Charge neutral receptors interacting with ligands via hydrogen bonding have for long been associated with poor compatibility with aqueous media. Presence of water and other protic solvents effectively disrupt the host-guest interactions in these systems. Recently however some exceptions to this rule have been reported although neutral receptors displaying affinity for phosphate and sulfate in pure water or buffer or as here investigated, high levels of salt, are rare. ${ }^{2}$ The concept of dual ion hosts suggests a way forward. ${ }^{9}$ These display high affinity and selectivity under physiological conditions by providing recognitive sites for both cation and anion in a geometry defined by the covalent chemistry used to link the two hosts. As shown in this work, template driven self assembly of the two hosts offers a straightforward alternative to construct these receptors. Combining macrocyclic cation hosts with neutral urea-based anion receptors can thus significantly boost ion affinity and capacity making these receptors a possible alternative for ion scavenging applications.

\section{Conflicts of interest}

There are no conflicts to declare.

\section{Acknowledgements}

We acknowledge financial support from the Marie SkłodowskaCurie Actions (H2020-MSCA-ITN-2016, 722171), Vetenskapsrådet (VR) under grant number 2014-3794 and the Sweden

Knowledge-Foundation (KKS) for research under project number 20150086. CW acknowledges support from KKSProspekt program under project number 20170040.

\section{Notes and references}

I This ion-pair imprinting approach contrasts with previously reported dual-ion imprinting of two or more cations. See: Prasad B., Jauhari D. and Verma A., Talanta, 2014, 120, 398-407.

1 Supramolecular chemistry in water, ed. S. Kubik, Wiley-VCH, 2019.

2 M. J. Langton, C. J. Serpell and P. D. Beer, Angew. Chem., Int. Ed. Engl., 2016, 55, 1974-1987.

3 P. Chakrabarti, J. Mol. Biol., 1993, 234, 463-482.

4 A. E. Hargrove, S. Nieto, T. Zhang, J. L. Sessler and E. V. Anslyn, Chem. Rev., 2011, 111, 6603-6782.

5 J. D. Watson and E. J. Milner-White, J. Mol. Biol., 2002, 315, 171-182.

6 I. Ravikumar and P. Ghosh, Chem. Soc. Rev., 2012, 41, 30773098.

7 P. M. Cummins, K. D. Rochfort and B. F. O'Connor, in Protein Chromatography: Methods and Protocols, ed. D. Walls and S. T. Loughran, Springer New York, New York, NY, 2017, pp. 209-223, DOI: 10.1007/978-1-4939-6412-3_11.

8 (a) D. Y. Sasaki, K. Kurihara and T. Kunitake, J. Am. Chem. Soc., 1992, 114, 10994-10995; (b) J. F. Neal, W. Zhao, A. J. Grooms, A. H. Flood and H. C. Allen, J. Phys. Chem. C, 2018, 122, 26362-26371.

9 (a) S. K. Kim and J. L. Sessler, Chem. Soc. Rev., 2010, 39, 37843809; (b) D. Smith, in Macrocyclic Chemistry: Current Trends and Future Perspectives, ed. K. Gloe, Springer Netherlands, Dordrecht, 2005, pp. 137-151, DOI: 10.1007/1-4020-36876_9.

10 M. M. G. Antonisse and D. N. Reinhoudt, Chem. Commun., 1998, 443-448, DOI: 10.1039/A707529D.

11 G. Wulff, T. Gross and R. Schönfeld, Angew. Chem., Int. Ed. Engl., 1997, 36, 1962-9164.

12 G. Wulff and K. Knorr, Bioseparation, 2002, 10, 257.

13 C. Lübke, M. Lübke, M. J. Whitcombe and E. N. Vulfson, Macromolecules, 2000, 33, 5098-5105.

14 M. Emgenbroich, C. Borrelli, S. Shinde, I. Lazraq, F. Vilela, A. J. Hall, J. Oxelbark, E. De Lorenzi, J. Courtois, A. Simanova, J. Verhage, K. Irgum, K. Karim and B. Sellergren, Chem.-Eur. J., 2008, 14, 9516-9529.

15 S. Shinde, A. Bunschoten, J. A. W. Kruijtzer, R. M. J. Liskamp and B. Sellergren, Angew. Chem., Int. Ed., 2012, 51, 83268329.

16 W. Wan, M. Biyikal, R. Wagner, B. Sellergren and K. Rurack, Angew. Chem., Int. Ed., 2013, 52, 7023-7027.

17 S. Shinde, Z. El-Schich, A. Malakpour, W. Wan, N. Dizeyi, R. Mohammadi, K. Rurack, A. Gjörloff Wingren and B. Sellergren, J. Am. Chem. Soc., 2015, 137, 13908-13912.

18 A. Cutivet, C. Schembri, J. Kovensky and K. Haupt, J. Am. Chem. Soc., 2009, 131, 14699-14702.

19 R. Sulc, G. Szekely, S. Shinde, C. Wierzbicka, F. Vilela, D. Bauer and B. Sellergren, Sci. Rep., 2017, 7, 44299. 
20 M. Liu, S. B. Torsetnes, C. Wierzbicka, O. N. Jensen, B. Sellergren and K. Irgum, Anal. Chem., 2019, 91, 1018810196.

21 P. Manesiotis, A. Riley and B. Bollen, J. Mater. Chem. C, 2014, 2, 8990-8995.

22 For examples of molecular recognition in water using MIPs see: (a) Y. Hoshino, T. Kodama, Y. Okahata and K. J. Shea, J. Am. Chem. Soc., 2008, 130, 15242-15243; (b) G. Pan, Y. Zhang, Y. Ma, C. Li and H. Zhang, Angew. Chem., Int. Ed., 2011, 50, 1-14; (c) F. Canfarotta, A. Poma, A. Guerreiro and S. Piletsky, Nat. Protoc., 2016, 11, 443.
23 J. Smid, S. C. Shah, R. Sinta, A. J. Varma and L. Wong, Pure Appl. Chem., 1979, 51, 111.

24 S. Wagner, C. Zapata, W. Wan, K. Gawlitza, M. Weber and K. Rurack, Langmuir, 2018, 34, 6963-6975.

25 C. J. Pedersen, J. Am. Chem. Soc., 1967, 89, 2495-2496.

26 D. J. Cram, Angew. Chem., Int. Ed. Engl., 1988, 27, 1009.

27 J. S. Bradshaw and R. M. Izatt, Acc. Chem. Res., 1997, 30, 338345.

28 Y. Takeda, Bull. Chem. Soc. Jpn., 1981, 54, 3133-3136. 



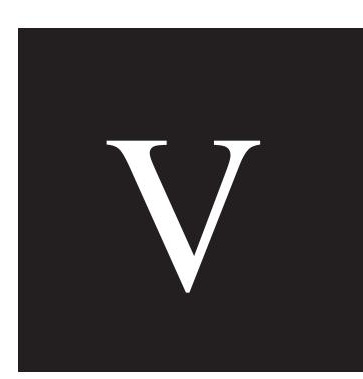





\title{
Imprinted Src-SH2 Domain Mimicking:
}

\section{Targeting pYEEI-sequence-containing peptides}

\author{
Anıl Incel, Börje Sellergren \\ ${ }^{1}$ Department of Biomedical Science, Faculty of Health and Society, Malmö University, 20506 Malmö, Sweden
}

\begin{abstract}
Phosphorylation of tyrosine controls many cellular functions. Profiling of SH2 (Src homology 2) which is a phospho-dependent-binding domain described as a modular-based diagnostics tool. One known SH2 ligand is pYEEl that is recognized by the Src-family of SH2 domain. In this work, PYEEI sequence is targeted for urea-based imprinting approach, which considered as crude mimics of SH2 domain recognizing.
\end{abstract}

\section{INTRODUCTION}

Protein phosphorylation is one of the most common reversible post translational modification (PTM) which is regulated by switch mechanism of kinases and phosphatases enzymes. ${ }^{1-4}$ This modification plays a pivotal role in the dynamic of cellular processes such as cell growth and differentiation. ${ }^{5-8}$ Particularly, phosphorylation on tyrosine $(\mathrm{pY})$ residues is of tremendous significance in phosphoproteome studies due to playing key roles in cellular signaling cascades. ${ }^{7,9,10}$ Tyrosine phosphorylation has exceptionally low abundance (1.8\%) when compares phosphorylation on serine (pS, $86.4 \%$ ) and threonine (pT, $11.8 \%$ ) residues in eukaryotic cells. ${ }^{11-14}$ Besides this small fraction of $\mathrm{pY}$, dysfunctions on both protein tyrosine kinases (PTKs) and phosphatases (PTPs) may lead to an abnormality on cellular transformations, and this unregulated event might cause some diseases such as cancer. ${ }^{15-18}$ Accordingly, it is crucial to find out and establish efficient methods for identification and/or enrichment of $\mathrm{pY}$ residues in the development of cancer diagnostics. However, notwithstanding their promising application in phosphoproteomics, the proposed alternative techniques, ${ }^{19-22}$ for instance, metal ion based affinity chromatography (IMAC, and MOAC) prior to mass spectrometry or bioanalytical methods like immunoprecipitation (IP) hinder their usage because of some disbenefits. Affinity-based approaches has deficiencies in discrimination on phosphorylated residues over non-phosphorylated ones and lack of selective preference among phosphorylated peptides such as pY, pS, pT. Antibody-like affinity requires large amount of initial material and show lack of specificity and selectivity on low immunogenic phosphorylation e.g. pS and pT and low coverage of $\mathrm{pY}^{23-26}$

For this reason, smart capture phases based on molecularly imprinted polymers (MIPs) is an emerging field of research, which fuses the advantages of synthetic molecular-recognitionelements with biological receptors, recently targeting higher molecular-weight analytes such as peptides and proteins as well. ${ }^{27-32}$ Many studies have been reported to develop phosphopeptide (p-peptide) selective MIPs. In terms of the levels of $p$-peptide discrimination, validation of phospho-residues over nonphosphorylated ones and identification of different phospho-sites using controlled bias materials increase the attention on imprinting technology.

Src homology 2 (SH2) domain, consisting of around 100 amino acid residues, is identified as sequence specific $\mathrm{pY}$ binding pocket which has 
connecting structure between PTKs and downstream of number amount of proteins. ${ }^{33-}$ ${ }^{38}$ The affinity of Src SH2 domain towards $\mathrm{pY}$ residues is strongly related with the amino acids motifs in the followed $p Y+1, p Y+2$ and $\mathrm{pY}+3$ positions placed in the C-terminal. ${ }^{38-40}$ Many studies on the screening of not only sequence specificity of $\mathrm{SH} 2$ domain in the recognition of pY-based polypeptides but also phosphorylation-site-dependent complexation have been determined binding affinity and specificity of Src-SH2 domain towards pY-based peptides and pY-oriented cell signaling. ${ }^{39,41}$ Therefore, pTyr-Glu-Glu-lle (pYEEI) sequence containing $p$-peptides had been identified as the optimal motif for binding of $\mathrm{Src} \mathrm{SH} 2$ domain with higher affinity in $\mathrm{nM}$ range. ${ }^{39}$ The specific interactions between $\mathrm{Src}-\mathrm{SH} 2$ domain and PYEEI sequence was shown in Scheme 1.

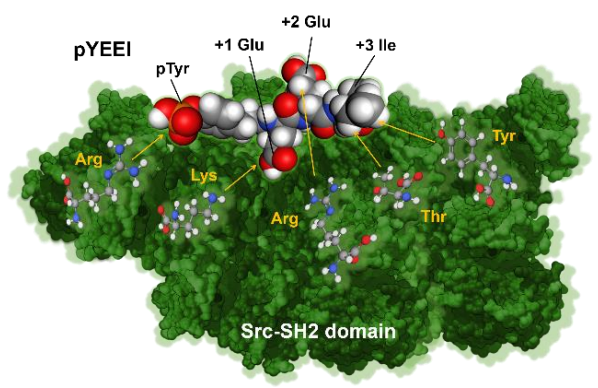

Scheme 1: The representative view of $\mathrm{SH} 2$ domain showing the specific binding sites and interactions with $\mathrm{pYEEI}$ sequence (pY-binding pocket has electrostatic interactions with Arginine (Arg), +1 Glu and +2 Glu has $\mathrm{H}$-bonding and electrostatic interactions with Lysine (Lys) and Arg, respectively, and +3 lle make hydrophobic interaction with aromatic ring of Tyr and methyl group of Threonine (Thr) of domain structure). The pYEEI peptide and interacted amino acids were shown as space-filling and ball-and-stick models, respectively, with colored as phosphor: orange, oxygen: red, nitrogen: blue, carbon: gray and hydrogen: white.

$\mathrm{SH} 2$ sepharose beads known as $\mathrm{SH} 2$ superbinder were used in some studies to mimic anti-pY antibodies and to enrich pTyr residues obtained from different cell lines. ${ }^{42-46}$ $\mathrm{SH} 2$ superbinder was used as bare ${ }^{43}$ or combined with $\mathrm{Ti}^{4+}-\mathrm{IMAC}^{44}$ to enhance the efficient enrichment of pTyr peptides. Moreover, microreactor coupled with $\mathrm{SH} 2$ superbinder was developed to enrich $p$ peptides from tiny amount of initial sample. ${ }^{46}$ In clinical cancer research, the elucidation of tyrosine kinase signaling pathways based on targeted phosphoproteomic has played a crucial role in identifying putative drug targets and the development of highly specific kinase inhibitor based anticancer agents. ${ }^{47} \mathrm{As}$ Src-SH2 domain-signaling protein is a great affinity reagent for the binding of specific $\mathrm{pY}$ modules, the mimicking of this domain using imprinting approach has a potential to develop robust and non-immunobased affinity-capture-material for the profiling of elusive phosphorylation.

In this study, we developed PYEEI sequencebased epitope-imprinted polymers in three different polymerization formats. Hydrogenbond-driven imprinting of oxyanions together with urea monomer approach ${ }^{48}$ made strong interactions and complexation between host and guest, which further enhanced the binding affinity and selectivity of prepared polymer materials toward target $\mathrm{p}$-peptide. This method gives full advantage of mimicking Src$\mathrm{SH} 2$ domain to enrich phosphorylated residues with high affinity ( $\mathrm{Kd}$ is in $\mu \mathrm{M}$ range) and preferably biased on EPQPYEEIPIYL peptide. The designed chemical receptors were also successfully applied to enrich pYEEI-sequencecontaining peptide from a complex mixture with an efficient selectivity.

\section{EXPERIMENTAL}

\section{Preparation of imprinted and non-imprinted}

polymers. $\mathrm{N}$ - and $\mathrm{C}$ - terminal protected phosphorylated and non-phosphorylated epitopes shown in Scheme $\mathbf{2}$ were used in the synthesis of imprinted polymers ( $\mathrm{pY}$ - and $\mathrm{Y}$ MIPs) together with urea based functional monomer, 1 using stoichiometric imprinting 1:2 and 1:4 complexation shown in Scheme 4. The control non-imprinted polymers (NIPs) were synthesized in the absence of these template molecules. 


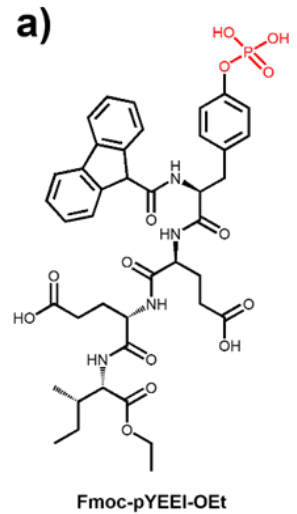

b)

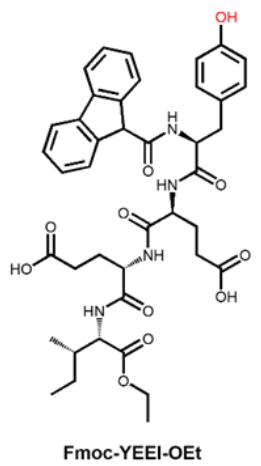

Scheme 2: Phosphorylated (a) and nonphosphorylated (b) N- and C- protected epitopes used in imprinting

High crosslinked (HC) polymers: The bistetrabutylammonium (bis-TBA) salt of Fmocprotected templates $(0.25,0.125) \mathrm{mmol}$ for the ratio of 1:2 (A) and 1:4(B) (T:FM), respectively), urea functional monomer, 1 (0.5 mmol), acrylamide, AAm (0.5 mmol) as co-monomer, pentaerythritol triacrylate, PETA (13.3 mmol) as crosslinker, acetonitrile, MeCN $(6.1 \mathrm{mmol})$ as porogen, and azobis (2,4-dimethyl) valeronitrile (ABDV) ( $1 \% \mathrm{w} / \mathrm{w}$ of total monomer amount) as initiator were used. The each prepared solution for the polymerization was purged with a flow of nitrogen gas for $15 \mathrm{~min}$. and the polymerization was initiated by placing the solution containers in a water bath heated to $50{ }^{\circ} \mathrm{C}$ for overnight. Each container was removed after polymerization and the polymers were crushed, and washed by $\mathrm{MeOH}: 1 \mathrm{~N} \mathrm{HCl}(1: 1 / \mathrm{v}: \mathrm{v}) \times 3$ times and $\mathrm{MeOH} \times 2$ times for the removal of template. This process was followed by sieving to obtain the particles between $25 \mu \mathrm{m}$ and $50 \mu \mathrm{m}$ to use the polymers for further analysis. Additionally, nonimprinted polymers were synthesized as same as above omitting template molecule.

High dilution, HD, polymers: The polymers were synthesized using the same protocol mentioned in the previous section except the volume of porogen used. In this time, the
MeCN amount was increased 20 times (122 $\mathrm{mmol})$. Then, the particles were precipitated with $\mathrm{MeOH}: \mathrm{H}_{2} \mathrm{O}(1: 1)$ and the same protocol carried out for template removal.

Microsphere, M, polymers: To prepare microsphere polymers (Scheme 3 ), surface modification of silica particles were completed. Firstly, $20.0 \mathrm{~g}$ of $\mathrm{NH}_{2} @ \mathrm{Si}$ was suspended in 100 $\mathrm{mL}$ of N,N-Dimethylformamide (DMF). Then, $20.0 \mathrm{~mL}$ of acetic anhydride was added and the suspension was stirred at room temperature overnight. N-acetylated silica (AcNH@Si) were obtained after particles were filtrated, washed with DMF $(3 \times 50 \mathrm{~mL})$ and $\mathrm{MeOH}(3 \times 50 \mathrm{~mL})$, dried under vacuum. The prepared AcNH@Si $(1.0 \mathrm{~g})$ were first deaerated in $50 \mathrm{~mL}$ Schlenk tubes (three cycles vacuum- $\mathrm{N}_{2}$ ) and then it allowed to soak with pre-polymerization mixture which, was prepared the same way as described in the synthesis of high cross-linked polymers, under continuous $\mathrm{N}_{2}$ flow while stirring with a spatula until the particles were freely flowing to make sure that the pore of particles were filled by mixture. Then, the tubes were closed with glass stoppers and the polymerization was initiated placing the tubes in water bath heated to $50{ }^{\circ} \mathrm{C}$. After $24 \mathrm{~h}$, the resulting composite beads were washed with 3 $\mathrm{M} \mathrm{NH}_{4} \mathrm{HF}_{2}$ aqueous solution to etch silica and then washed by $\mathrm{MeOH}: 1 \mathrm{~N} \mathrm{HCl}(1: 1 / \mathrm{v}: \mathrm{v}) \times 3$ times and $\mathrm{MeOH} \times 2$ times for the removal of template.

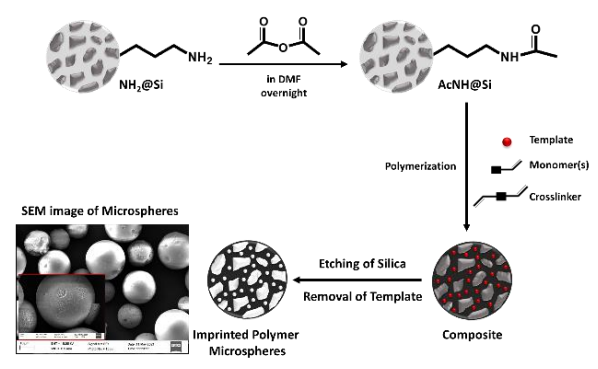

Scheme 3. Preparation of microsphere polymers consisting of acetylation of silica, copolymerization in silica scaffold followed by etching and template removal. 


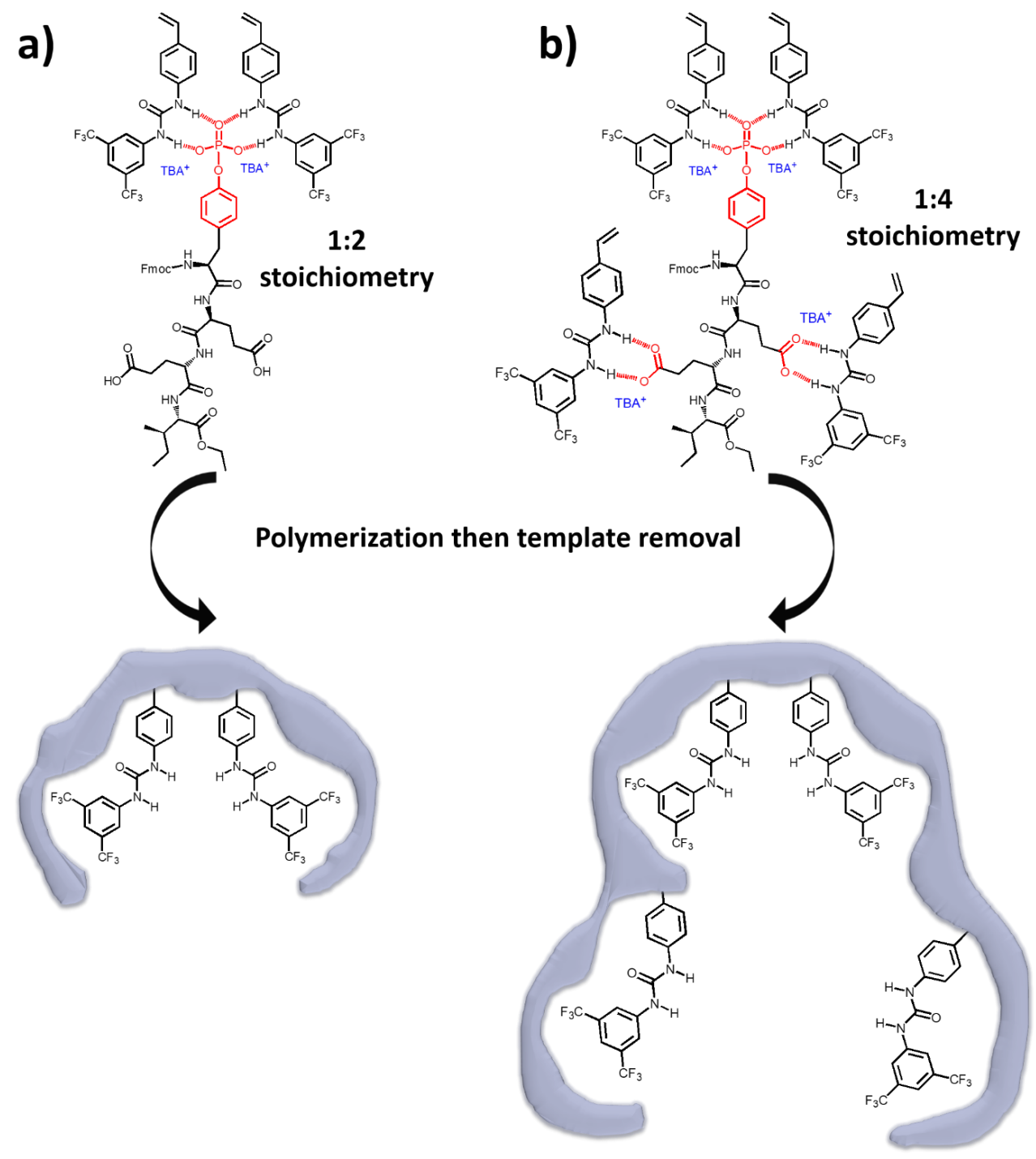

Scheme 4. Stoichiometric imprinting using N- and C- protected templates with functional monomer 1. a. 1:2 and b. 1:4 template:monomer complexation.

Equilibrium binding tests. Each polymer (5.0 $\mathrm{mg}$ ) was suspended in $1.0 \mathrm{~mL}$ of a mixture of equimolar pYEEI (EPQpYEEIPIYL) and YEEI (EPQYEEIPIYL) (each $20 \mu \mathrm{M}$ ) prepared in organic based $95 \%$ MeCN buffered with $0.1 \%$ TFA and $0.1 \%$ TEA modifiers and aqueous buffers at different $\mathrm{pHs}(\mathrm{pH}=1$ (hydrochloric acid in water), $\mathrm{pH}=3$ and 5 (sodium citrate), $\mathrm{pH}=7.4$ (HEPES), and $\mathrm{pH}=9.2$ (sodium carbonate)) and then later on $80 \% \mathrm{MeCN}+0.1 \%$ TFA:20\% HEPES buffer $(\mathrm{pH}=7.4)$ Each suspension was shaken vigorously for $2 \mathrm{~h}$ and then centrifuged. The supernatant $(400 \mu \mathrm{L})$ was dried using Genevac EZ-2 evaporator, then reconstituted with $200 \mu \mathrm{L}$ of $30 \% \mathrm{MeCN}+0.1 \%$ TFA prior to analysis using reversed phase HPLC. The column was Prodigy $5 \mu \mathrm{m}$ ODS-3 100 $\AA$ (Phenomenex, $150 \times 4.6 \mathrm{~mm}$ ). Mobile phases 
were (A) $\mathrm{H}_{2} \mathrm{O}+0.1 \%$ TFA and (B) $\mathrm{MeCN}+0.1 \%$ TFA. A linear gradient method of $30 \%$ B to $40 \%$ $B$ in $10 \mathrm{~min}$ at a flow rate of $1.0 \mathrm{~mL} / \mathrm{min}$. was used. The injection volume was $50.0 \mu \mathrm{L}$ and the detection was performed by UV absorbance measurement at $220.0 \mathrm{~nm}$. All experiments were performed in three parallel replicas.

Binding equilibrium. The polymers ( $5 \mathrm{mg}$ each) were separately mixed with $500 \mu \mathrm{L}$ of pYEEI (EPQpYEEIPIYL) and YEEI (EPQYEEIPIYL) peptide at $1,2,5,10,25$, and $50 \mu \mathrm{M}$ concentration prepared in three different conditions $195 \%$ MeCN+0.1\% TFA, $80 \%$ $\mathrm{MeCN}+0.1 \%$ TFA:20\% HEPES, and HEPES aqueous buffer, $\mathrm{pH}=7.4$ ) and shaken overnight for equilibrium followed by centrifuged; the supernatant was dried and reconstituted. The samples were analyzed by reverse phase HPLCUV using the same method described in the previous section. Each experiment was performed three times. The binding curves were constructed by plotting the graph $B$ ( $\mu \mathrm{mol} / \mathrm{g}$ ) corresponding to free concentration, Cfree, and were subsequently fitted in the GraphPad Prism 7 software (GraphPad Software, La Jolla, CA, USA) by Langmuir monosite binding model in the following formula, which is $Y=B_{\max } * X /\left(K_{d}+X\right)$.

\section{Extraction of target p-peptide and analysis.}

Preparation of beta-casein digest: Beta-casein ( $\beta$-casein from bovine milk, $10 \mathrm{mg}$ ) was dissolved in $100 \mathrm{mM} \mathrm{NH}_{4} \mathrm{HCO}_{3}(1 \mathrm{~mL})$ was reduced with $50 \mathrm{mM}$ DTT for $30 \mathrm{~min}$ at $50{ }^{\circ} \mathrm{C}$, and alkylated with $100 \mathrm{mM}$ IAA for $30 \mathrm{~min}$ at room temperature in the dark. Then, the protein was digested with trypsin $(200 \mu \mathrm{g})$ for $24 \mathrm{~h}$ at $37^{\circ} \mathrm{C}$. The sample was then acidified with TFA and desalted on a C18 silica gel column (Bond Elut C18, Anlaytichem International, CA, USA). The column was first activated with $\mathrm{MeCN}+0.1 \%$ TFA $(2 \mathrm{~mL})$ and conditioned with $0.1 \%$ TFA $(2 \times 2 \mathrm{~mL})$. Finally, the peptides were eluted with $80 \%$
$\mathrm{MeCN}+0.1 \%$ TFA $(2 \mathrm{~mL})$, the digest was lyophilized and stored at $-20^{\circ} \mathrm{C}$ prior to use.

Sequence specificity test using longer peptides. The mixture of eight phosphorylated and non-phosphorylated peptides, which are EPQYEEIPIYL, EPQPYEEIPIYL, DRVYIHPF, DRVPSIHPF, DRVPYIHPF, GADDSYYTAR, GADDSPYYTAR, and GADDSPYPYTAR, was tested with polymers. Followed loading/washing conditions were carried out for each individual $\mathrm{pY}$-imprinted and corresponding non-imprinted polymers: HC: HEPES aqueous buffer, $\mathrm{pH}=7.4, \mathrm{HD}: 80 \%$ MeCN+0.1\% TFA:20\% HEPES, M: 95\% MeCN + $0.1 \%$ TFA. Then the peptides were eluted with three consecutive incubations with $500 \mu \mathrm{L}$ of $50 \% \mathrm{MeOH}, 80 \% \mathrm{MeOH}$, and $99.9 \% \mathrm{MeOH}$ buffered with $0.1 \%$ TFA. Fractions were analyzed by MALDI MS/MS TOF.

\section{Enrichment of target p-peptide from complex}

mixture. Src-SH2 domain peptides, pYEEI (EPQPYEEIPIYL) and YEEI (EPQYEEIPIYL) were mixed together in an equimolar mixture (400 pmol each) and then mixing together with $\beta$-casein digest (10 nmol): spiking level is 1:25. Peptide mixture $(500 \mu \mathrm{L}$ prepared in $1.0 \mathrm{~mL}$ Low-binding Eppendorf tube) was incubated with $5 \mathrm{mg}$ of promising polymers (where showed preference on targeted $\mathrm{pY}$ over nonphosphorylated one) prepared at different conditions (95\% MeCN + 0.1\% TFA, $80 \%$ MeCN+0.1\% TFA:20\% HEPES, and HEPES aqueous buffer, $\mathrm{pH}=7.4$ ) for $3 \mathrm{~h}$. After incubation, each tube was centrifuged and the supernatant were collected as flow-though fraction and labelled as FT. Next, each polymer was washed for $30 \mathrm{~min}$ with loading solvent (2 $\times 250 \mu \mathrm{L}$ ) and the supernatant after centrifuge was collected as washing fraction, and combined together with flow-through and labelled as $\mathrm{FT}+\mathrm{W}$. Then, the peptides were eluted from polymers with three consecutive incubations with $500 \mu \mathrm{L}$ of $50 \% \mathrm{MeOH}, 80 \%$ 
$\mathrm{MeOH}$, and $99.9 \% \mathrm{MeOH}$ all buffered with $0.1 \%$ TFA for $30 \mathrm{~min}$. The supernatants were pooled together as elution fraction and labelled as $E$. Both $\mathrm{FT}+\mathrm{W}$ and $\mathrm{E}$ fractions were dried by using Genevac EZ-2 evaporator. Then each fraction was analyzed by both HPLC-UV ESI-MS and MALDI-MS/MS TOF and the results were compared with pre-enrichment fraction in terms of abundance of spiked peptides and identification of number of peptides in each fraction, respectively.

HPLC ESI-MS analysis. The HPLC ESI-MS analyses were performed in the reversed phase mode using an analytical C18 column (Prodigy $5 \mu \mathrm{m}$ ODS-3 $100 \AA$ A , Phenomenex, $150 \times 4.6$ $\mathrm{mm})$. The mobile phases were (A) $\mathrm{H}_{2} \mathrm{O}+0.1 \%$ TFA and (B) MeCN+0.1\% TFA. A linear gradient method of $30 \%$ B to $40 \%$ B in 10 min at a flow rate of $1.0 \mathrm{~mL} / \mathrm{min}$. was used. The injection volume was $50.0 \mu \mathrm{L}$ and the detection was performed by UV absorbance measurement at $220.0 \mathrm{~nm}$ and electrospray mass spectrometry (ESI-MS) selective ion monitoring (SIM) with the mass range of $1.0 \mathrm{Da}$. The mass spectra were recorded in positive ion mode. The analysis parameters were set as follows: capillary voltage $5 \mathrm{kV}$, cone voltage $30 \mathrm{~V}$, source temperature $120^{\circ} \mathrm{C}$, and desolvation temperature $360^{\circ} \mathrm{C}$.

MALDI-TOF MS/MS analysis. The aliquots of the collected flow-through, washing and elution fractions were dried by using Genevac EZ-2 evaporator. The residue was dissolved in $\mathrm{H}_{2} \mathrm{O}+0.1 \%$ TFA $(20 \mu \mathrm{L})$. The $1 \mu \mathrm{L}$ of matrix solution and $1 \mu \mathrm{L}$ of concentrated SPE aliquots were deposited together on the target plate. Mass-spectrometric analysis of the fractions was performed using a MALDI reflector timeof-flight mass spectrometer (Ultraflex mass spectrometer, Bruker-Daltonics $\mathrm{GmbH}$, Bremen, Germany) equipped with a Scout-384 source in positive reflector mode unless otherwise stated. For analysis of samples, DHB matrix was prepared by dissolving $30 \mathrm{mg}$ DHB in $1 \mathrm{~mL}$ of $50 \% \mathrm{MeCN}$ and $1 \%$ phosphoric acid $+0.1 \%$ TFA. Each condition was identically kept same in terms of scanning conditions and number of scans. The spectra was collected by accumulating 1000 laser shots under reflective mode (relative laser focus: $50 \%$, global attenuator offset: $66 \%$, detector gain: $1805 \mathrm{~V}$, sample rate and digitizer: $1.0 \mathrm{GS} / \mathrm{s}$, ion source 1 and 2: $25.0 \mathrm{kV}$ and $22.0 \mathrm{kV}$, lens: $8.0 \mathrm{kV}$, reflector 1 and 2: $26.3 \mathrm{kV}$ and $14.7 \mathrm{kV}$ ) and further analyzed with the Flexanalysis 3.0 software (Bruker Daltonics). The mass spectra were analyzed with flex Control software (Brucker Daltonic).

\section{RESULTS and DISCUSSION}

Synthesized polymers by different formats. The aim on this study is to synthesize chemical binders using imprinting approach to extract particular phosphorylation on tyrosine in sequence specific manner from complex mixture. Regarding to our hypothesis, we synthesized imprinted polymers using phosphorylated and non-phosphorylated templates and their complementary nonimprinted polymers for control testing at three different formats, which are high cross-linked, high-dilution and microspheres. HC polymers were synthesized as monolithic porous materials with broader distribution of pore size in the presence of minimum amount of porogen. This traditional method lacks of micro-size inner pores showing accessibility issue for larger-sized peptides. HD polymers were prepared in the presence of excess amount of porogen, which is a type of precipitation polymerization technique, using highly diluted monomer solution that allows forming narrower distribution of micro- and nano- sized porous structure in the end material. $\mathbf{M}$ polymers were synthesized using acetylated silica particles as solid support, which were soaked by polymerization mixture to produce controlled pore size beads. 
Stoichiometric Imprinting. In the design of chemical receptors via imprinting approach, the complex formation and stoichiometry between monomer and template play the key role for further favorable recognition on target. The preferred monomer in this study was 1,3disubstituted neutral urea host providing 2fold hydrogen bond donor with the presence of basic oxyanions such as phosphate as an acceptor. To maximize the strengths on complexation, bulky ammonium ions, TBA.OH (tetrabutylammonium hydroxide) is used to make base salt form of phosphate-oriented template, not only enhances the interactions due to providing more solubility but also provides better noticeable binding sites for memory effect.

Regarding to the phosphorylated template used in imprinting, which is Fmoc-pTyr-GluGlu-lle-OEt, two different stoichiometry was introduced in the synthesis considering the complexation between monourea host and guest. Ternary complex as 1:2 phosphate:urea and possible quinary complex as 1:4 stand for one, 1:2 phosphate:urea and two, 1:1 carboxylate:urea were used in the synthesis of imprinted polymers (Scheme 3).

Probing affinity and selectivity of polymers in peptide mixture. In order to probe the affinity of polymers to recognize phosphorylated peptide, PYEEl, each polymer was tested with the mixture of -pYEEI- and -YEEI- peptides prepared in $95 \%$ MeCN buffered with either acidic, $0.1 \%$ trifluoroacetic acid (TFA) or basic, $0.1 \%$ triethylamine (TEA) modifier. The selectivity ratio towards PYEEI over YEEI was given in Figure 1. The acidic condition in binding showed a strong preference in the retention of PYEEl target peptide over nonphosphorylated form, while basic condition led approximately equal preference for both peptides (Fig. 1). Considerably, $\mathrm{pH}$ of binding solution together with acidic modifier $(0.1 \%$ TFA, pKa $=0.5$ ) provides partial negative charge
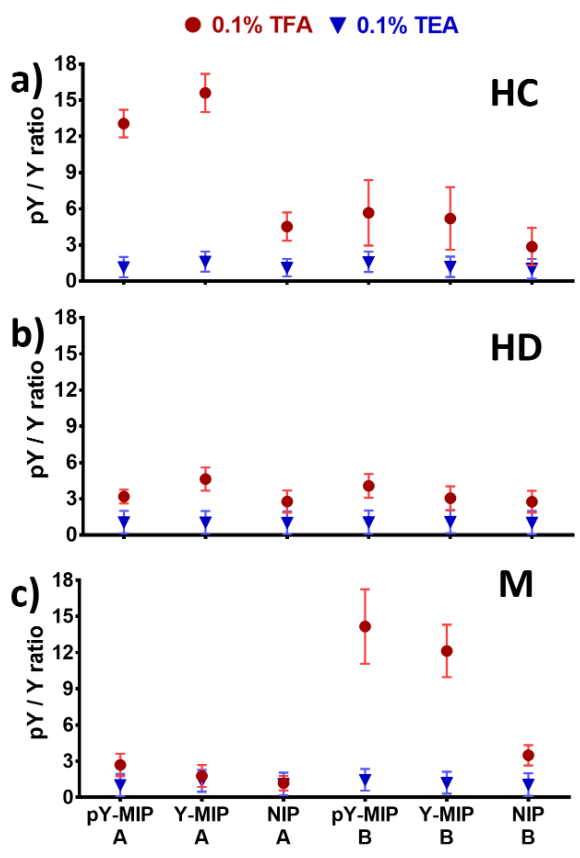

Figure 1. The binding selectivity ratio of $p Y$ peptide over $\mathrm{Y}$ peptide after incubated with imprinted and non-imprinted high-crosslinked, $\mathrm{HC}(\mathrm{a})$, high-dilution, $\mathrm{HD}(\mathrm{b})$ and microsphere, $\mathrm{M}$ (c) polymers in 95\% MeCN buffered with $0.1 \%$ TFA and $0.1 \%$ TEA as indicated. ( $p Y$ and $Y$ imprinted polymers using phosphorylated and non-phosphorylated templates and $A$ and $B$ refers to $1: 2$ and 1:4 - T:FM ratio, respectively, used in the polymerization).

on phosphate anion (pKa,1=0.9) and fully protonation on carboxylate group (pKa,1=4.2). Hence, with the basic modifier (0.1\% TEA, $\mathrm{pKa}=10.7)$, quadruple hydrogen bonds forms with urea group due to fully deprotonated of phosphate anion, and the -1 charge state of carboxylate group, additionally promotes nonspecific interactions together with non-target peptide, YEEI. Moreover, high cross-linked imprinted polymers synthesized by 1:2 T:FM ratio and polymer microspheres synthesized by 1:4 T:FM ratio present pronounced selectivity on PYEEI than the other imprinted ones. This preference remarks substantial memory on phospho-motif used in polymerization step. Even though high-dilution imprinted polymers show higher uptake of both peptides -due to 
the fact that this polymerization technique increase the swelling capacity of gel-like formed particles and enhance the binding percent of analytes- (Fig. S1, see adsorbed amount), the preference on PYEEI peptide was much lower compared to other imprinting formats.

After confirming selectivity of imprinted polymers towards target phosphotyrosine containing peptide in water-poor media, each polymer was tested in aqueous buffers prepared at different $\mathrm{pH}=1.0,3.0,5.0,7.4$, and 9.2. Most of synthetic receptors targeting oxyanions lack of effective recognition in water media since anions are highly hydrated and hydrogen bonding with host gets weaker. In terms of designed neutral-urea based receptors together with hydrophilic crosslinker, PETA was used to increase the wettability of particles aiming better stabilization between targets and imprinting sites. The selectivity ratio towards PYEEI over YEEI after testing each polymer at aqueous buffers prepared at different $\mathrm{pH}$ was given in Figure 2.

The binding of target pYEEl peptide at highly and slightly acidic $\mathrm{pH}(1.0,3.0$, and 5.0) was equal or lower -except at $\mathrm{pH}=3.0, \mathrm{~A}$ series of HD polymers- than YEEI peptide compared to neutral $(\mathrm{pH}=7.4)$ and basic $(\mathrm{pH}=9.2)$ environments. At $\mathrm{pH}=1.0$, both peptides were bound non-specifically to imprinted and nonimprinted polymers without any difference among formats due to very hydrophobic form of peptides in highly acidic aqueous buffer (Fig. S2). At $\mathrm{pH}=5$, the binding of nonphosphorylated peptide, YEEI, was found much higher than PYEEl target peptide by all polymers - except A series of HD polymers since phosphate group is carrying one charged oxygen (pKa, $2=6.3$ ) while carboxylate group is fully deprotonated, which promotes binding of YEEI peptide under this condition. -because phosphate-containing peptide has charged form that makes it more hydrophilic and competing between water and for binding sites more than non-phosphorylated peptideNevertheless, at neutral $\mathrm{pH}=7.4$ and slightly basic $\mathrm{pH}=9.2$, some of imprinted polymers showed promising preference on target peptide. At $\mathrm{pH}=7.4$, pY-MIP-B-HC and pY-MIP$A-H D$ polymers revealed better retention on PYEEI peptide. PY-MIP-A-M selectivity on target enhances with increase on $\mathrm{pH}$ but the selectivity remains insignificant (Fig. 2).
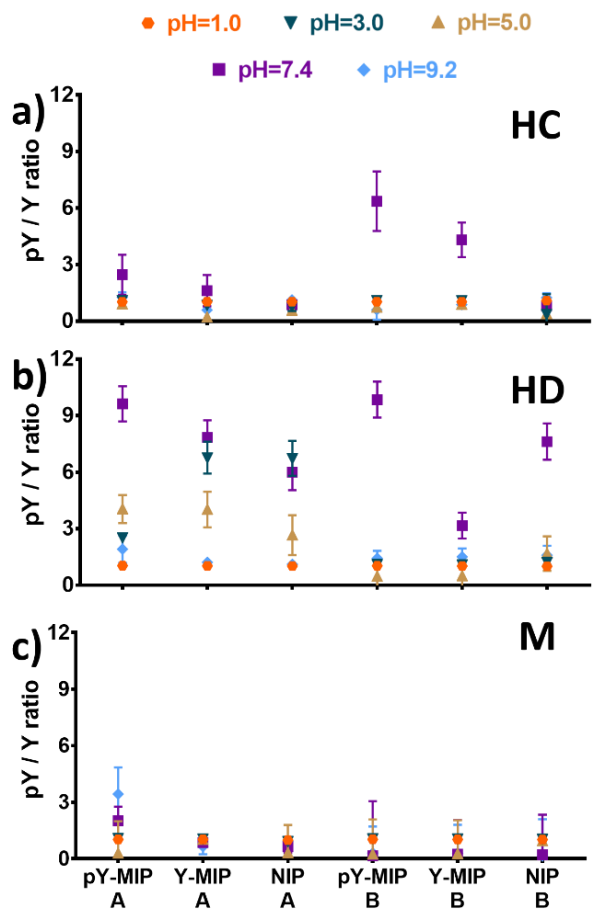

Figure 2: The binding selectivity ratio of pY peptide over $Y$ peptide after incubated with imprinted and non-imprinted high-crosslinked, $\mathrm{HC}(\mathrm{a})$, high-dilution, HD (b) and microsphere, $\mathrm{M}$ (c) polymers in aqueous buffers at different $\mathrm{pH}$ indicated as 1.0, 3.0, 5.0, 7.4, and 9.2. ( $\mathrm{pY}$ and $\mathrm{Y}$ imprinted polymers using phosphorylated and non-phosphorylated templates and $A$ and $B$ refers to $1: 2$ and 1:4 - T:FM ratio, respectively, used in the polymerization).

Comparing a water-poor media such as $95 \%$ $\mathrm{MeCN}+0.1 \%$ TFA with aqueous buffers, the memory effect of solvent used in polymerization and significant role of hydrophilic crosslinking agent to enhance bias 
on target binding in aqueous buffers were confirmed. Then, aqueous buffer together with $\mathrm{MeCN}+0.1 \%$ TFA was used for batch binding. Regarding to binding profile of HD polymers in both aprotic solvent rich media with modifiers and aqueous buffers (especially at $\mathrm{pH}=7.4$ ), the optimal water-poor media was prepared using $80 \% \mathrm{MeCN}+0.1 \%$ TFA together with $20 \%$ HEPES buffer $(\mathrm{pH}=7.4)$. The aim of using this loading solution was to make $\mathrm{pH} \sim 3.0$ enabling acidic amino acids protonated while phosphate is sufficiently deprotonated for binding. The presence of $80 \% \mathrm{MeCN}$ allows decreasing nonspecific binding -where YEEI peptide is more hydrophobic, in this case- $20 \%$ buffer provides better solubility, moreover, the low diffusion coefficient might help neutralize charges and this sulfonic acid buffering agent mimics phosphate group to reduce non-specific interactions. The mixture of PYEEI and YEEI peptides prepared in $80 \% \mathrm{MeCN}+0.1 \%$ TFA: $20 \%$ HEPES $(\mathrm{pH}=7.4)$ was incubated with $A$ series of polymers.

The batch binding results showed that while the selectivity on PYEEI increased using imprinted $\mathrm{HD}$ polymers; imprinted $\mathrm{HC}$ and $\mathrm{M}$ polymers' bias on PYEEI decreased with lower adsorbed amount (Fig. S3a). The selectivity ratio of polymers on target was compared after tested in $95 \% \mathrm{MeCN}+0.1 \% \mathrm{TFA}$ and $80 \% \mathrm{MeCN}$ $+0.1 \%$ TFA : $20 \%$ HEPES (Fig. 3Sb) which showed that the preference of pYEEI peptide using imprinted HD polymers were ameliorated under this condition - the others were diminished. Lastly, B series of HD polymers were tested (Fig. S3C) and slight -not encouraging- increase on PYEEl preference was obtained.

After probing the selectivity on target pYEEI peptide at different binding conditions using polymers synthesized with different formats, three pY-imprinted, complementary nonimprinted polymers, and binding solutions where pronounced bias on PYEEI- were chosen for further experiments.
Determination of binding capacity $\left(\mathrm{B}_{\max }\right)$ and the dissociation constant $\left(K_{d}\right)$. Binding isotherm profiles of the chosen polymers, which are $\mathrm{pY}$-imprinted HC-B, HD-A, and M-B and their NIPs, were recorded. The isotherms for $\mathrm{pY}$-imprinted polymers showed a great fit to the Langmuir mono-site binding model for the determination of homogeneous binding sites. Applying the curve fitting model each system (pY-MIP or NIP : pYEEI or YEEI), the binding isotherms exhibited different profile properties regarding to trend, slope, and saturation point (Fig. 3).

- pY-MIP:pYEEI - pY-MIP:YEEI
-. NIP:pYEEI $\quad \cdots$ NIP:YEEI
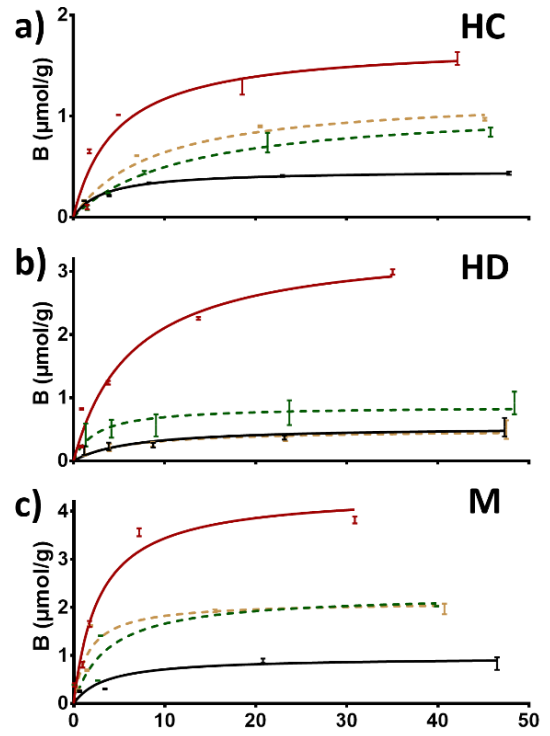

Figure 3. The binding isotherms of PYEEI and YEE peptides after treated with $B$ series of $\mathrm{HC}$ (a, prepared in HEPES buffer $(\mathrm{pH}=7.4))$, A series of HD (b, prepared in $80 \% \mathrm{MeCN}+0.1 \%$ TFA:20\% HEPES buffer $(\mathrm{pH}=7.4)$ ) and $B$ series of $M$ (c, prepared in $95 \% \mathrm{MeCN}+0.1 \%$ TFA) pY-template imprinted and non-imprinted polymers as indicated in the inlet. The concentration range is from $2 \mu \mathrm{M}$ to $50 \mu \mathrm{M}$. The binding parameters were obtained by fitting of the binding data to a Langmuir mono-site binding model.

The binding properties was given in Table 1. The binding isotherms of pY-imprinted polymers towards PYEEI peptide displayed a 
much steeper slope with a saturation points at 1.72( \pm 0.3$), 3.46( \pm 0.4)$, and $4.41( \pm 0.4) \mu \mathrm{mol} / \mathrm{g}$, for $H C, H D$, and $M$ format, respectively. Analyzing the curves assuming the fitting model gave $K_{d}$ values as $\mu M$ level for $p Y-$ MIP:pYEEI, exhibiting a higher affinity for target peptide. On the other hand, $\mathrm{pY}$-imprinted polymers towards YEEI peptide and NIPs for both peptides presented shallower curves reaching saturation point immediately.

Table 1. Binding properties of target PYEEI peptide after treated with $\mathrm{pY}$-template imprinted polymers $(\mathrm{Kd}$, the dissociation constant, Bmax, maximum binding capacity regarding to the amount of sorbent used, and R2, coefficient of determination after one site-specific binding fitting model used).

\begin{tabular}{cccc}
\hline MIP : pYEEI & $\mathrm{K}_{\mathrm{d}}\left(10^{6}\right)$ & $\mathrm{B}_{\max }(\mu \mathrm{mol} / \mathrm{g})$ & $\mathrm{R}^{2}$ \\
& $\mathrm{M}$ & & \\
\hline pY-HC-B : pYEEI & $4.69( \pm 2.5)$ & $1.72( \pm 0.3)$ & 0.89 \\
\hline pY-HD-A : pYEEI & $6.42( \pm 2.5)$ & $3.46( \pm 0.4)$ & 0.95 \\
\hline pY-M-B : pYEEI & $2.79( \pm 0.9)$ & $4.41( \pm 0.4)$ & 0.96 \\
\hline
\end{tabular}

Probing affinity and selectivity of polymers where PYEEI and YEEI peptides spiked in betacasein digest. Having proven the binding and affinity of $\mathrm{pY}$-imprinted polymers towards pYEEl target peptide, further investigation was carried out to understand the ability of binders to enrich target p-peptide from complex mixture. For this aim, phosphorylated and nonphosphorylated $\mathrm{SH} 2$ domain peptides were spiked in beta-casein digest at 1:25 spiking level. The fractions obtained from enrichment experiments were analyzed using HPLC ESI-MS and the extracted chromatograms of preenrichment, $\mathrm{FT}+\mathrm{W}$ (flow-through+washing) and $E$ (elution) fractions were represented in Figure $\mathbf{4}$ (MIPs) and Figure $\mathbf{5}$ (NIPs). Moreover, the pre-enrichment and elution fractions were also screened using MALDI-MS to profiles of sample complexity and peptides that were shown in Figure 6.

First, we noted that sample complexity in $\mathrm{E}$ fraction compared to pre-enrichment and $\mathrm{FT}+\mathrm{W}$ fraction was reduced due to elimination
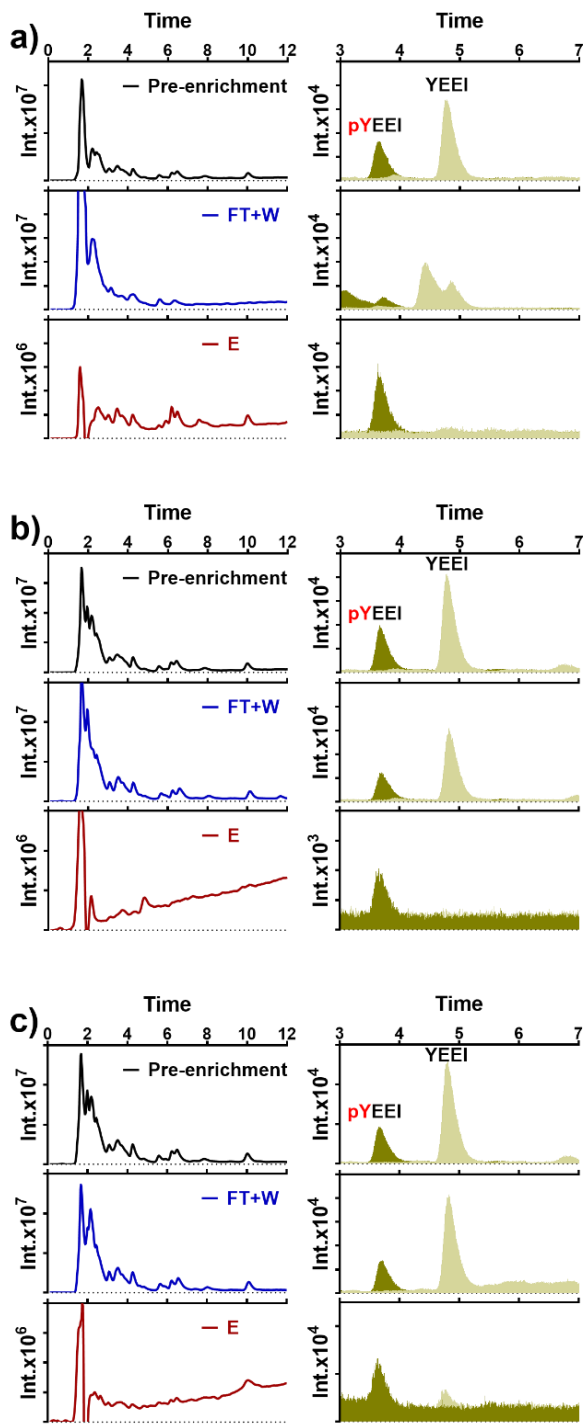

Figure 4. HPLC-UV chromatograms (left) and corresponding ESI-MS (right) selective ion monitoring (SIM, $\mathrm{m} / \mathrm{z}=1473.51$ for pYEEI and $\mathrm{m} / \mathrm{z}=1393.51$ for YEEI peptides) spectra of pre-enrichment, flowthrough and washing $(\mathrm{FT}+\mathrm{W})$ and consecutive pooled elution (E) fractions obtained from a. pY-MIP-HC-B, b. pY-MIP-HD-A, c. pY-MIP-M-B, after loading a sample of digested beta-casein (10000 pmol) spiked with pYEEI $(400 \mathrm{pmol})$ and YEEI $(400 \mathrm{pmol})$. The mass profile of $\mathrm{pYEEI}$ and YEEI peptides were indicated.

of non-specific contributions of peptides in complex digest. Moreover, most of YEEI peptide was found in $\mathrm{FT}+\mathrm{W}$ fractions obtained using pY-MIPs. The abundance of target $p$ - 

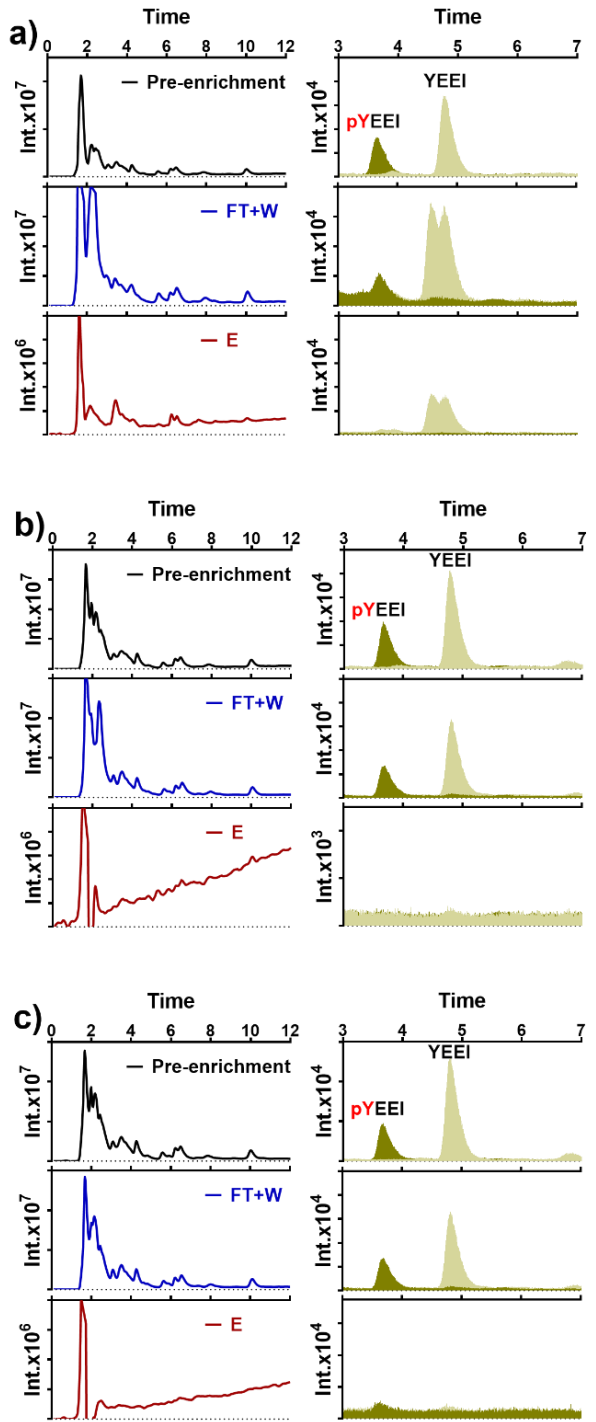

Figure 5. HPLC-UV chromatograms (left) and corresponding ESI-MS (right) selective ion monitoring (SIM, $\mathrm{m} / \mathrm{z}=1473.51$ for pYEEI and $\mathrm{m} / \mathrm{z}=1393.51$ for YEEI peptides) spectra of pre-enrichment, flowthrough and washing $(\mathrm{FT}+\mathrm{W})$ and consecutive pooled elution (E) fractions obtained from a. NIP-HC-B, b. NIP-HD-A, c. NIP-M-B, after loading a sample of digested beta-casein (10000 pmol) spiked with pYEEI $(400 \mathrm{pmol})$ and YEEI $(400 \mathrm{pmol})$. The mass profile of PYEEI and YEEI peptides were indicated.

peptide over non-phosphorylated one in $E$ fraction obtained from pY-MIPs in Fig. 4 and from NIPs in Fig. 5 showed promising selective enrichment of target pYEEI peptide via pY- imprinted polymers. In the comparison of pYMIPs in different formats for preference on target peptide, pY-MIP-M-B (Fig. 6c, E: pY-MIP$M-B$ ) exhibited clearer enrichment of target with eliminated background peaks compared to pY-MIP-HC-B (Fig. 6a, E: pY-MIP-HC-B) and pY-MIP-HD-A (Fig. 6b, E: pY-MIP-HD-A). These experiments confirm the possibility of extract target p-peptide from complex mixture.

Apart from capturing phosphorylated target over non-phosphorylated one, the other levels of selectivity was further investigated testing pY-MIPs and NIPs with peptide mixture containing different not only single but also multiple phosphorylated and nonphosphorylated peptides with serine and tyrosine residues (Fig. 7a). The sequence of peptides with $[\mathrm{M}+\mathrm{H}]^{+}$value and the motif which might possibly interact with polymers also given as table in Figure 7a. The enrichment was completed with mixture of eight peptides and peptide profile was screened using MALDIMS. The elution fractions from $p Y-M I P s$ and NIPs were shown in Figure 7b-d. While pY-MIPM-B showed clear pick up of target EPQpYEEIPIYL peptide -which was presented as $[\mathrm{M}+\mathrm{H}]^{+}$and $[\mathrm{M}+\mathrm{Na}]^{+}$adduct-, pY-MIP-HC-B and $\mathrm{PY}$-MIP-HD-A showed cross-reactivity with multiply phosphorylated tyrosine peptide, GADDSPYPYTAR. On the other hand, while NIPHC-B exhibited non-specific interaction with target peptide but also it enriches other single or multiple phosphorylated peptides; NIP-HD$A$ and NIP-M-B resulted similar profiles showing higher abundance on single phosphorylated serine and tyrosine angiotensin peptides. The comparison of all fractions showed that imprinted polymers synthesized using PYEEI motif presented sequence specific binding towards target $p$ peptide in the presence of other phosphorylated and non-phosphorylated peptides with similar motifs. This particular study confirms the possible sequence specific enrichment of target peptide using MIPs. 


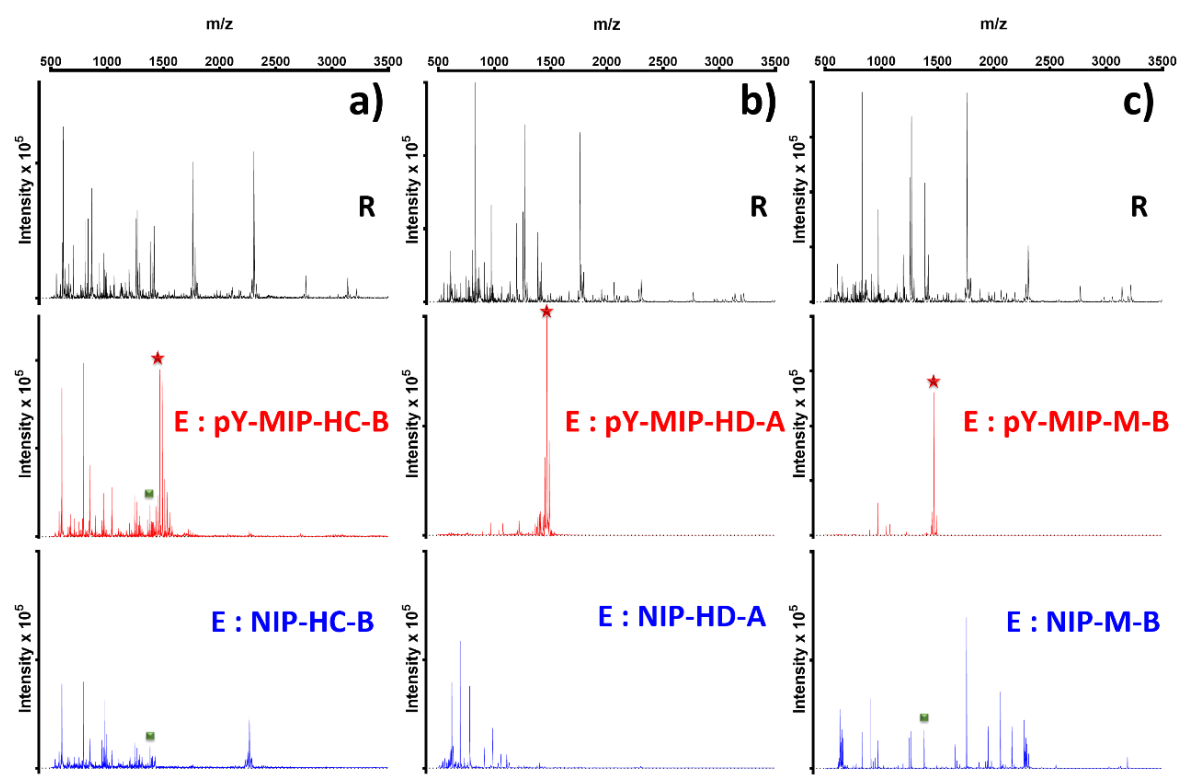

Figure 6. MALDI-MS spectra obtained for peptide mixture before enrichment (1st row), consecutive pooled elution fractions from pY-MIPs (2nd row), and corresponding NIPs (3rd row) from a. HC, b. HD and c. M. Target spiked peptide PYEEI $(\mathrm{M}+\mathrm{H}+=1473.51)$ (asterisk) and non-target spiked peptide YEEI $(\mathrm{M}+\mathrm{H}+=1394.51)$ (square) were marked in elution fractions.
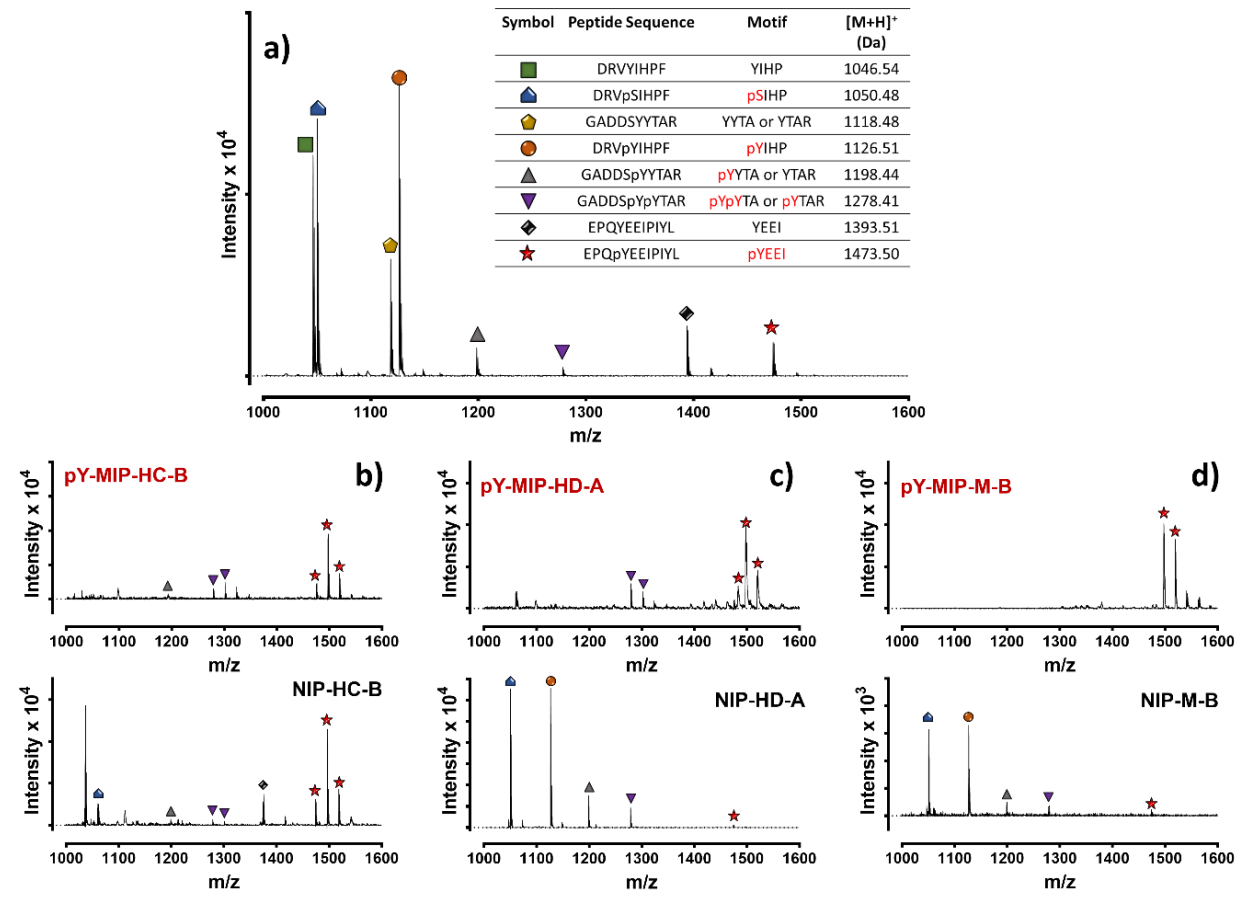

Figure 7. MALDI-MS spectra obtained for peptide mixture before enrichment, a. reference together with property table, consecutive pooled elution fractions from pY-MIPs (top), and corresponding NIPs (bottom) from b. HC, c. HD and d. M. 


\section{OUTLOOK and FUTURE}

SH2 domains are known as phosphorylationespecially pTyr containing peptides and sequence specific- dependent variants that play critical role in receptor signaling and signal transduction pathways. Src-SH2 domain specifically bind the pYEEI-sequence containing peptides with higher affinity in $\mathrm{nM}$ range. In this work, synthesized $\mathrm{pY}$-imprinted polymers in different formats showed promising affinity on target p-peptide that can mimic the binding of Src-SH2 domain. Additionally, promising sequence specific enrichment of PYEEIsequence containing peptide in the mixture of other phosphorylated and non-phosphorylated peptides would lead great potential on targeted phosphoproteomics especially development of highly specific kinase inhibitors in the future. This application can be achieved expanding research on extraction of natural target from cell lysate, optimizing enrichment conditions and applying this study into clinical phosphoproteomics.

\section{AUTHOR INFORMATION}

\section{Corresponding Author}

*E-mail: borje.sellergren@mau.se

*E-mail: anil.incel@mau.se

\section{ACKNOWLEDGMENT}

This work was supported by the EU-funded Marie Curie ITN project H2020-MSCA-ITN2016, 722171-BioCapture.

\section{REFERENCES:}

(1) Blackstock, W. P.; Weir, M. P. Trends in Biotechnology. 1999.

(2) Jensen, O. N. Nature Reviews Molecular Cell Biology. 2006.

(3) Ubersax, J. A.; Ferrell, J. E. Nature Reviews Molecular Cell Biology. 2007.

(4) Morandell, S.; Stasyk, T.; Grosstessner-Hain, K.; Roitinger, E.; Mechtler, K.; Bonn, G. K.; Huber, L. A. Proteomics. 2006.

(5) Reinders, J.; Sickmann, A. Proteomics. 2005.
(6) Andersen, J. N.; Mortensen, O. H.; Peters, G. H.; Drake, P. G.; Iversen, L. F.; Olsen, O. H.; Jansen, P. G.; Andersen, H. S.; Tonks, N. K.; Møller, N. P. H. Mol Cell Biol 2001.

(7) Hunter, T. Current Opinion in Cell Biology. 2009.

(8) Lim, W. A.; Pawson, T. Cell. 2010

(9) Hunter, T. Cold Spring Harb Perspect Biol 2014.

https://doi.org/10.1101/cshperspect.a020 644.

(10) Alonso, A.; Sasin, J.; Bottini, N.; Friedberg, I.; Friedberg, I.; Osterman, A.; Godzik, A.; Hunter, T.; Dixon, J.; Mustelin, T. Cell. 2004.

(11) Sharma, K.; D'Souza, R. C. J.; Tyanova, S.; Schaab, C.; Wiśniewski, J. R.; Cox, J.; Mann, M. Cell Rep 2014.

(12) Nishi, H.; Shaytan, A.; Panchenko, A. R. Frontiers in Genetics. 2014.

(13) Ardito, F.; Giuliani, M.; Perrone, D.; Troiano, G.; Muzio, L. Lo. International Journal of Molecular Medicine. 2017

(14) Schwartz, P. A.; Murray, B. W. Bioorganic Chemistry. 2011.

(15) Harsha, H. C.; Pandey, A. Molecular Oncology. 2010.

(16) Blume-Jensen, P.; Hunter, T. Nature. 2001.

(17) Sawyers, C. L. Current Opinion in Genetics and Development. 2002.

(18) Rush, J.; Moritz, A.; Lee, K. A.; Guo, A.; Goss, V. L.; Spek, E. J.; Zhang, H.; Zha, X. M.; Polakiewicz, R. D.; Comb, M. J. Nat Biotechnol 2005.

(19) Thingholm, T. E.; Jensen, O. N.; Larsen, M. R. Proteomics. 2009.

(20) Fílla, J.; Honys, D. Amino Acids. 2012.

(21) Leitner, A. In Methods in Molecular Biology; 2016.

(22) Mann, M.; Ong, S. E.; Grønborg, M.; Steen, H.; Jensen, O. N.; Pandey, A. Trends in Biotechnology. 2002.

(23) Zoumaro-Djayoon, A. D.; Heck, A. J. R.; Muñoz, J. Methods 2012.

(24) van der Mijn, J. C.; Labots, M.; Piersma, S. R.; Pham, T. V.; Knol, J. C.; Broxterman, H. J.; 
Verheul, H. M.; Jiménez, C. R. J Proteomics 2015.

(25) Ding, V. M. Y.; Boersema, P. J.; Foong, L. Y.; Preisinger, C.; Koh, G.; Natarajan, S.; Lee, D. Y.; Boekhorst, J.; Snel, B.; Lemeer, S.; et al. PLoS One 2011.

(26) Di Palma, S.; Zoumaro-Djayoon, A.; Peng, M.; Post, H.; Preisinger, C.; Munoz, J.; Heck, A. J. R. J Proteomics 2013.

(27) Mosbach, K.; Ramström, O. Bio/Technology. 1996.

(28) Wulff, G. Angewandte Chemie International Edition in English. 1995.

(29) Haginaka, J.; Tabo, H.; Matsunaga, H. Anal Chim Acta 2012.

(30) Emgenbroich, M.; Borrelli, C.; Shinde, S.; Lazraq, I.; Vilela, F.; Hall, A. J.; Oxelbark, J.; De Lorenzi, E.; Courtois, J.; Simanova, A.; et al. Chem - A Eur J 2008.

(31) Chen, J.; Shinde, S.; Subedi, P.; Wierzbicka, C.; Sellergren, B.; Helling, S.; Marcus, K. J Chromatogr A 2016.

(32) Helling, S.; Shinde, S.; Brosseron, F.; Schnabel, A.; Müller, T.; Meyer, H. E.; Marcus, K.; Sellergren, B. Anal Chem 2011.

(33) Sadowski, I.; Stone, J. C.; Pawson, T. Mol Cell Biol 1986.

(34) Moran, M. F.; Koch, C. A.; Anderson, D.; Ellis, C.; England, L.; Martin, G. S.; Pawson, T. Proc Natl Acad Sci U S A 1990.

(35) Pawson, T.; Gish, G. D. Cell. 1992.

(36) Waksman, G.; Kominos, D.; Robertson, S. C.; Pant, N.; Baltimore, D.; Birge, R. B.; Cowburn, D.; Hanafusa, H.; Mayer, B. J.; Overduin, M.; et al. Nature 1992.

(37) Waksman, G.; Shoelson, S. E.; Pant, N.; Cowburn, D.; Kuriyan, J. Cell 1993.

(38) Waksman, G.; Kuriyan, J. Cell. 2004.

(39) Zhou, S.; Shoelson, S. E.; Chaudhuri, M.; Gish, G.; Pawson, T.; Haser, W. G.; King, F.; Roberts, T.; Ratnofsky, S.; Lechleider, R. J.; et al. Cell 1993.

(40) Huang, H.; Li, L.; Wu, C.; Schibli, D.; Colwill, K.; Ma, S.; Li, C.; Roy, P.; Ho, K.; Songyang, Z.; et al. Mol Cell Proteomics 2008.

(41) Boggon, T. J.; Eck, M. J. Oncogene. 2004.
(42) Ke, A. Q.; Liu, A. D.; Gao, Y. N.; Luo, D. N.; Li, Z. F.; Yu, Y. Q.; Liu, J. Y.; Xu, H.; Cao, X. Anal Chim Acta 2018.

(43) Yao, Y.; Wang, Y.; Wang, S.; Liu, X.; Liu, Z.; Li, Y.; Fang, Z.; Mao, J.; Zheng, Y.; Ye, M. J Proteome Res 2019.

(44) Bian, Y.; Li, L.; Dong, M.; Liu, X.; Kaneko, T.; Cheng, K.; Liu, H.; Voss, C.; Cao, X.; Wang, Y.; et al. Nat Chem Biol 2016.

(45) Tong, J.; Cao, B.; Martyn, G. D.; Krieger, J. R.; Taylor, P.; Yates, B.; Sidhu, S. S.; Li, S. S. C.; Mao, X.; Moran, M. F. Proteomics 2017.

(46) Yao, Y.; Bian, Y.; Dong, M.; Wang, Y.; Lv, J.; Chen, L.; Wang, H.; Mao, J.; Dong, J.; Ye, M. J Proteome Res 2018.

(47) Machida, K.; Khenkhar, M.; Nollau, P. Genes and Cancer 2012.

(48) Shinde, S.; Incel, A.; Mansour, M.; Olsson, G. D.; Nicholls, I. A.; Esen, C.; Urraca, J.; Sellergren, B. J Am Chem Soc 2020. 


\title{
Supporting Information
}

\section{Imprinted Src-SH2 Domain Mimicking:}

\section{Targeting pYEEI-sequence-containing peptides}

\author{
Anıl Incel, Börje Sellergren
}

Table of content:

Figure S1: Adsorbed amount of pYEEI and YEEI peptide by imprinted and non-imprinted polymers in $95 \%$ MeCN buffered with either $0.1 \%$ TFA $(a, b, c)$ or $0.1 \%$ TEA ( $d, e, f)$ modifier. HC, HD and $M$ stand for high crosslinked, high-dilution, microsphere polymers where $\mathrm{pY}$ and $\mathrm{Y}$ imprinted polymers using phosphorylated and non-phosphorylated templates and $\mathrm{A}$ and $\mathrm{B}$ refers to $1: 2$ and 1:4 - T:FM ratio, respectively, used in the polymerization.

Figure S2: Adsorbed amount of pYEEI and YEEI peptide by imprinted and non-imprinted polymers in aqueous buffers at different $\mathrm{pH}=1.0,3.0,5.0,7.4,9.2$. $\mathrm{HC}(\mathrm{a}), \mathrm{HD}(\mathrm{b})$ and $\mathrm{M}(\mathrm{c})$ stand for high crosslinked, high-dilution, microsphere polymers where $\mathrm{pY}$ and $\mathrm{Y}$ imprinted polymers using phosphorylated and non-phosphorylated templates and $\mathrm{A}$ and $\mathrm{B}$ refers to $1: 2$ and 1:4 - T:FM ratio, respectively, used in the polymerization.

Figure S3: Absorbed amount of pYEEI and YEEI peptides after incubation in $80 \% \mathrm{MeCN}+0.1 \%$ TFA:20\% HEPES (pH=7.4) by imprinted and non-imprinted polymers prepared in different formats as indicated (a), the comparison of binding selectivity ratio of $\mathrm{pY}$ peptide over $\mathrm{Y}$ peptide with imprinted and non-imprinted $\mathrm{A}$ series of high-crosslinked, HC, high-dilution, HD and microsphere, $\mathrm{M}$ polymers prepared in $95 \% \mathrm{MeCN}+0.1 \% \mathrm{TFA}$ (left) and $80 \% \mathrm{MeCN}+0.1 \% \mathrm{TFA}: 20 \% \mathrm{HEPES}$ (right) as indicated, and the selectivity ratio of $\mathrm{pY}$ over $\mathrm{Y}$ peptide tested by all $\mathrm{M}$ polymers and compared binding selectivity in two different conditions as indicated (c) ( $\mathrm{pY}$ and $\mathrm{Y}$ imprinted polymers using phosphorylated and non-phosphorylated templates and $\mathrm{A}, \mathrm{B}, \mathrm{C}$ refers to $1: 2$ and 1:4 - T:FM ratio, respectively, used in the polymerization). 


\section{Q pYEEI peptide \\ $\because$ YEEI peptide}

$0.1 \%$ TFA modifier
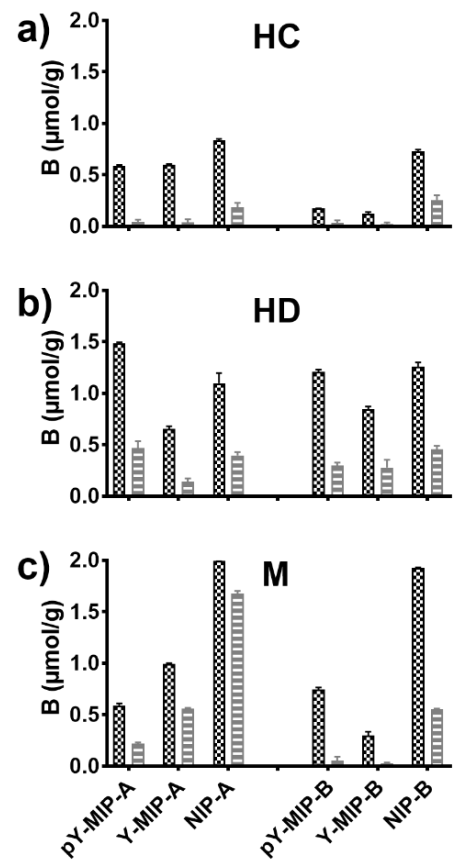

$0.1 \%$ TEA modifier
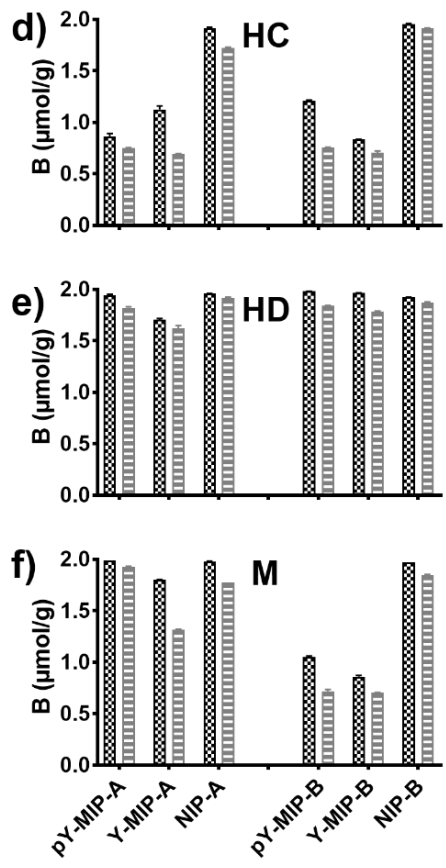

Figure S1: Adsorbed amount of pYEEI and YEEI peptide by imprinted and non-imprinted polymers in 95\% MeCN buffered with either $0.1 \% \operatorname{TFA}(a, b, c)$ or $0.1 \%$ TEA (d, e, f) modifier. HC, HD and M stand for high crosslinked, high-dilution, microsphere polymers where $\mathrm{pY}$ and $\mathrm{Y}$ imprinted polymers using phosphorylated and non-phosphorylated templates and A and B refers to 1:2 and 1:4 - T:FM ratio, respectively, used in the polymerization. 


\section{Q pYEEI peptide}

\section{YEEI peptide}
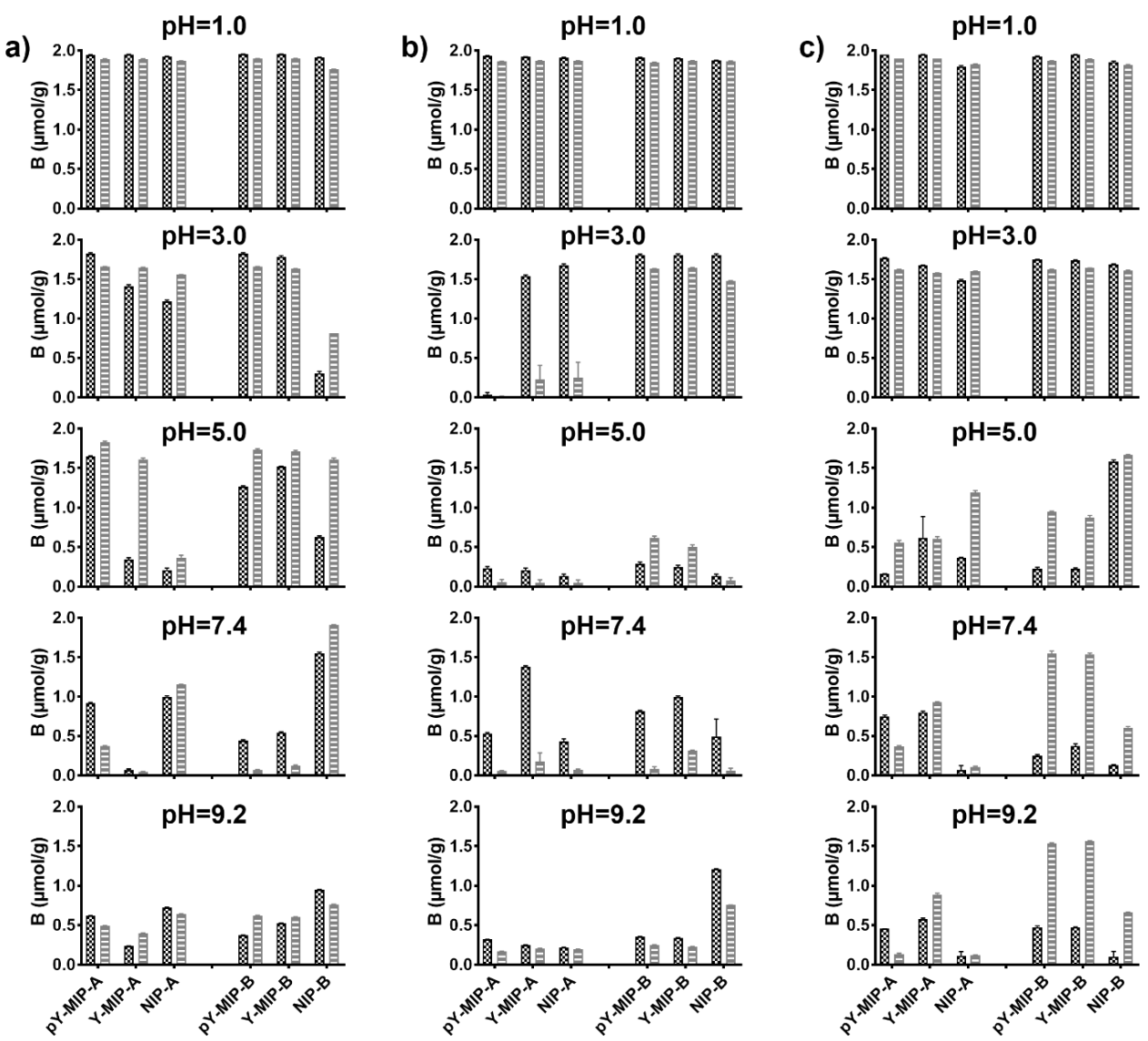

Figure S2: Adsorbed amount of pYEEI and YEEI peptide by imprinted and non-imprinted polymers in aqueous buffers at different $\mathrm{pH}=1.0,3.0,5.0,7.4,9.2$. $\mathrm{HC}(\mathrm{a}), \mathrm{HD}(\mathrm{b})$ and $\mathrm{M}$ (c) stand for high crosslinked, high-dilution, microsphere polymers where $\mathrm{pY}$ and $\mathrm{Y}$ imprinted polymers using phosphorylated and non-phosphorylated templates and $A$ and $B$ refers to 1:2 and 1:4 - T:FM ratio, respectively, used in the polymerization. 
a) $\triangle$ pYEEI peptide $\equiv$ YEEl peptide
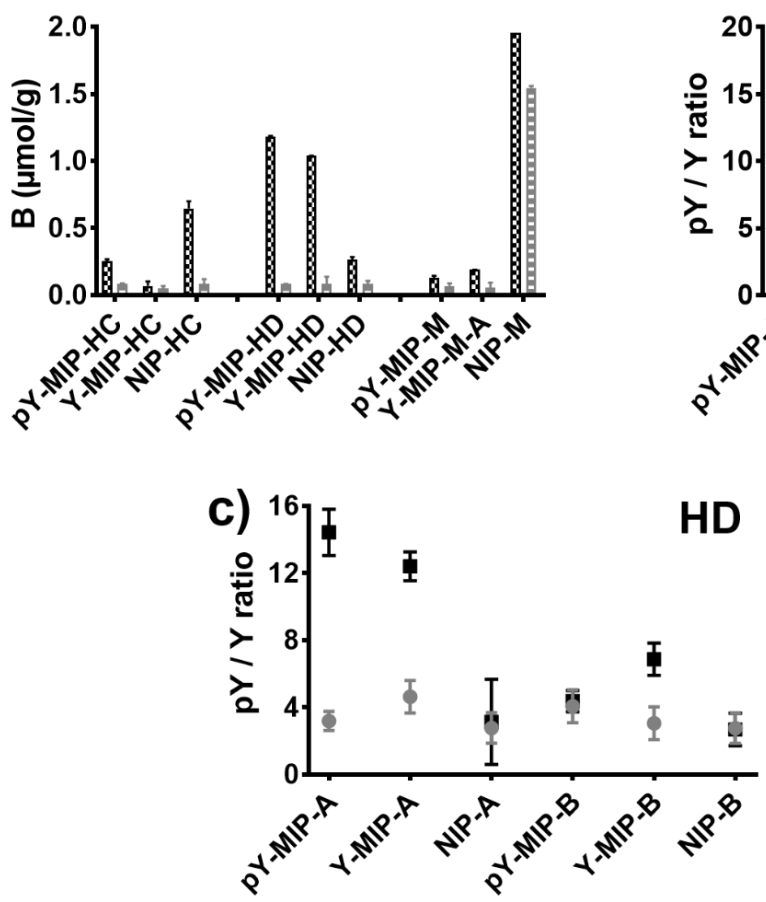

b)

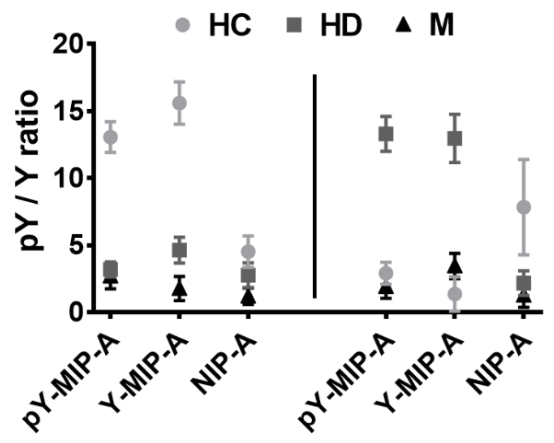

- $95 \% \mathrm{MeCN}+0.1 \%$ TFA

- $80 \% \mathrm{MeCN}+0.1 \%$ TFA

Figure S3: Absorbed amount of pYEEl and YEEl peptides after incubation in $80 \% \mathrm{MeCN}+0.1 \%$ TFA:20\% HEPES $(\mathrm{pH}=7.4)$ by imprinted and non-imprinted polymers prepared in different formats as indicated (a), the comparison of binding selectivity ratio of $\mathrm{pY}$ peptide over $\mathrm{Y}$ peptide with imprinted and nonimprinted $\mathrm{A}$ series of high-crosslinked, $\mathrm{HC}$, high-dilution, $\mathrm{HD}$ and microsphere, $\mathrm{M}$ polymers prepared in $95 \% \mathrm{MeCN}+0.1 \%$ TFA (left) and $80 \% \mathrm{MeCN}+0.1 \%$ TFA:20\% HEPES (right) as indicated (b), and the selectivity ratio of $\mathrm{pY}$ over $\mathrm{Y}$ peptide tested by all $\mathrm{M}$ polymers and compared binding selectivity in two different conditions as indicated (c) ( $p Y$ and $Y$ imprinted polymers using phosphorylated and nonphosphorylated templates and $A, B, C$ refers to $1: 2$ and 1:4 - T:FM ratio, respectively, used in the polymerization). 


\section{Malmö University Health and Society Doctoral Dissertations}

Ross, M. W. Typing, doing and being. A study of men who have sex with men and sexuality on the Internet. 2006:1

Stoltz, P. Searching for meaning of support in nursing. A study on support in family care of frail aged persons with examples from palliative care at home. 2006:2

Gudmundsson, P. Detection of myocardial ischemia using real-time myocardial contrasts echocardiograpy. 2006:3

Holmberg, L. Communication in palliative home care, grief and bereavement. A mother's experiences. 2007:1

Ny, P. Swedish maternal health care in a multiethnic society - including the fathers. 2007:2

Schölin, T. Etnisk mångfald som organisationsidé. Chefs- och personalpraktiker i äldreomsorgen. 2008:1

Svensson, O. Interactions of mucins with biopolymers and drug delivery particles. 2008:2

Holst, M. Self-care behaviour and daily life experiences in patients with chronic heart failure. 2008:3

Bahtsevani, C. In search of evidence-based practices. Exploring factors influencing evidence based practice and implementation of clinical practice guidelines. 2008:4

Andersson, L. Endocytosis by human dendritic cells. 2009:1

Svendsen, I. E. In vitro and in vivo studies of salivary films at solid/liquid interfaces. 2009:2

Persson, K. Oral health in an outpatient psychiatric population. Oral status, life satisfaction and support. 2009:3

Hellman, P. Human dendritic cells. A study of early events during pathogen recognition and antigen endocytosis. 2009:4

Baghir-Zada, R. Illegal aliens and health (care) wants. The cases of Sweden and the Netherlands. 2009:5

Stjernswärd, S. Designing online support for families living with depression. 2009:6

Carlsson, A. Child injuries at home - prevention, precautions and intervention with focus on scalds. 2010:1

Carlson, E. Sjuksköterskan som handledare. Innehåll i och förutsättningar för sjuksköterskors handledande funktion i verksamhetsförlagd utbildning - en etnografisk studie. 2010:2

Sinkiewicz, G. Lactobacillus reuteri in health and disease. 2010:3

Tuvesson, H. Psychiatric nursing staff and the workplace. Perceptions of the ward atmosphere, psychosocial work environment, and stress. 2011:1

Ingvarsdotter, K. Mental ill health and diversity. Researching human suffering and resilience in a multicultural context. 2011:2 
Hamit-Eminovski, J. Interactions of biopolymers and metal complexes at biological interfaces. 2011:3

Mellgren, C. What's neighbourhood got to do with it? The influence of neighbourhood context on crime and reactions to crime. 2011:4

Annersten Gershater, M. Prevention of foot ulcers in patients with diabetes mellitus. Nursing in outpatient settings. 2011:5

Pooremamali P. Culture, occupation and occupational therapy in a mental care context- the challenge of meeting the needs of Middle Eastern immigrants. 2012:1

Gustafsson A. Aspects on sepsis: treatment and markers. 2012:2

Lavant, E. Multiplex HLA-DR-DQ genotyping. For genetic epidemiology and clinical risk assessment. 2012:3

Wangel, A-M. Mental ill-health in childbearing women. Markers and risk factors. 2012:4

Scaramuzzino, R. Equal opportunities? - A cross-national comparison of immigrant organisations in Sweden and Italy. 2012:5

Ivert, A-K. Adolescent mental health and utilisation of psychiatric care - The role of parental country of birth and neighbourhood of residence 2013:1

Znamenskaya, Y. Effect of hydration on thermodynamic, rheological and structural properties of mucin. 2013:2

Andersson, F. The female offender. Patterning of antisocial and criminal activity over the lifecourse. 2013:3

Lindroth, M. Utsatthet och sexuell hälsa - en studie om unga på statliga ungdomshem. 2013:4

Hulusjö, A. The multiplicities of prostitution experience - narratives about power and resistance. 2013:5

Falk, M. Direct electron transfer based biofuel cells. Operation in vitro and in vivo. 2014:1

Finnbogadóttir, H. Exposure to domestic violence during pregnancy. Impact on outcome, midwives' awareness, women's experience and prevalence in the south of Sweden. 2014:2

Fagerström, A. Effects of surfactant adjuvants on barrier properties of plant leaf cuticle. 2014:3

Lamberg, P. Design and characterization of direct electron transfer based biofuel cells including tests in cell cultures. 2014:4

Richert, T. Överdoser, försörjningsstrategier och riskhantering - livsvillkor för personer som injicerar narkotika. 2014:5

Örmon, K. Experiences of abuse during the life course. - Disclosure and the care provided among women in a general psychiatric context. 2014:6

Sjöblom, I. Planerade hemförlossningar i Norden - kvinnors och barnmorskors perspektiv. 2014:7

Albèr, C. Humectants and Skin - Effects of hydration from molecule to man. 2015:1 
Kisch M., A. Allogeneic stem cell transplantation. - Patients' and sibling donors' perspectives. 2015:2

Weiber, I. Children in families where the mother has an intellectual or developmental disability - incidence, support and first person perspectives. 2015:3

Schlyter, M. Myocardial infarction, Personality factors, Coping strategies, Depression and Secondary prevention 2016:1

Carlström, C. BDSM - Paradoxernas praktiker. 2016:2

El-Schich, Z. Novel imaging technology and tools for biomarker detection in cancer. 2016:3

Boonsatean, W. Living with type 2 diabetes in Thai population: Experiences and socioeconomic characteristics. 2016:4

Vejzovic, V. Going through a colonoscopy and living with inflammatory bowel disease: Children's and parents' experiences and evaluation of the bowel cleansing quality prior to colonoscopy. 2016:5

Isma, G.E. Overweight and obesity in young children: Preventive work in child health care with focus on nurses' perceptions and parental risk factors. 2016:6

Brännvall, M. Frigörelse med förhinder - Om polisanmälan när kvinnor tar sig ur mäns våld i nära relationer. 2016:7

Pankratov, D. Self-charging biosupercapacitors. 2016:8

Guidi, P. Social work assessment of families with children at risk: Similarities and differences in Italian and Swedish public social services. 2016:9

Jakobsson, J. The process of recovery after colorectal cancer surgery: Patients' experiences and factors of influence. 2017:1

Gerell, M. Neighborhoods without community. Collective efficacy and crime in Malmö, Sweden. 2017:2

Wierzbicka, C. New fractionation tools targeting elusive post-translational modifications. 2017:3

Afzelius, M. Families with parental mental illness: Supporting children in psychiatric and social services. 2017:4

Nordgren, J. Making drugs ethnic - Khat and minority drug use in Sweden. 2017:5

Nilsson, E-L. Parental socialization and adolescent offending. 2017:6

Sixtensson, J. Härifrån till framtiden. Om gränslinjer, aktörskap och motstånd i tjejers vardagsliv. 2018:1

Vasiljevic, Z. Ambulatory risk assessment and intervention in the prison services. Using Interactive Voice Response to assess and intervene on acute dynamic risk among prisoners on parole. 2018:2

González Arribas, E. Flexible and transparent biological electric power sources based on nano-structured electrodes. 2018:3 
Svalin, K. Risk assessment of intimate partner violence in a police setting. Reliability and predictive accuracy. 2018:4

Andersson, M. Hate crime victimization: consequences and interpretations. 2018:5

Djampour, P. Borders crossing bodies. The stories of eight youth with experience of migrating 2018:6

Yeung, S.Y. Stimuli-responsive lipid bilayer mimics for protein, virus and cell recognition. 2018:7

Holst-Hansson, A. On a journey for survival: everyday life during radiation therapy from the perspectives of women with breast cancer and their families. 2018:8

Berlin Hallrup, L. Experiences of Everydaylife and Participation for People with Intellectual Disabilities -From four Perspectives. 2019:1

Aleksejeva, O. Blue copper proteins as bioelements for bioelectronics devices 2019:2

Wendel, L. Dokumentation, profession och hälso- och sjukvård. Rättsliga perspektiv. 2019:3

Larsson, H. Existentiell ensamhet hos sköra äldre personer: ett närståendeperspektiv. 2020:1

Sundström, M. Existentiell ensamhet hos sköra äldre personer: Vårdpersonals och volontärers erfarenheter och behov av stöd. 2020:2.

Kvist, M. Varken resurs eller problem. Om lågutbildade ungas etablerings- och försörjningsmöjligheter. 2020:3.

Waldie, S. Model Membranes and Their Interactions with Native and Artificial Lipoproteins 2020:4

Sjöberg, M. Existentiell ensamhet hos sköra äldre personer: Äldre personers upplevelser samt dokumentation i patientjournalen. 2020:5

Samzelius, T. A vicious circle of silent exclusion: Family homelessness and poverty in Sweden from a single-mother perspective. 2020:6

Hylén, M. Pain in Intensive Care: assessments and patients' experiences. 2021:1

Engström, A. Everyday life, crime, and fear of crime among adolescents and young adults. 2021:2

Ali, A. Topical formulations, design and drug delivery: A dive into water. 2021:3

Mavliutova, L. Molecularly imprinted micro- and nanoparticles for cancer associated glycan motifs. 2021:4

Incel, A. Amino acid sequence and side chain specific synthetic receptors targeting protein phosphorylation. 2021:5

The publications are available on-line.

https://mau.diva-portal.org/ 



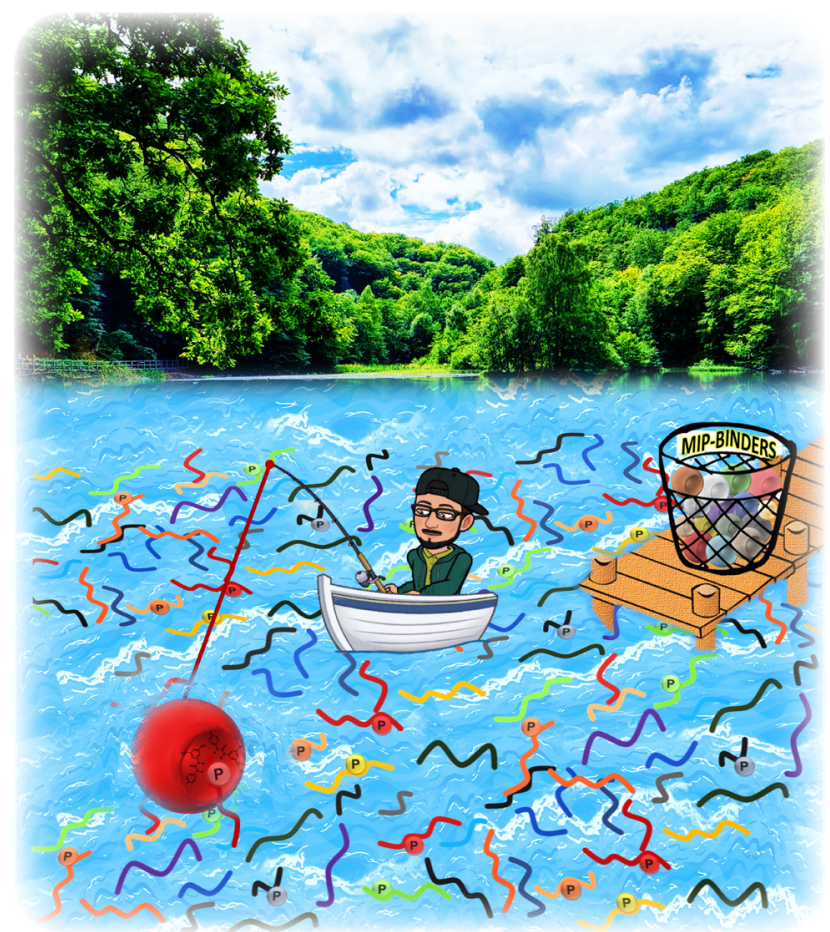

ISBN 978-91-7877-207-0 (print) ISBN 978-91-7877-208-7 (pdf) ISSN 1653-5383 